\title{
AN INNOVATIVE METHODOLOGY FOR ALLOCATING RELIABILITY AND COST IN A LUNAR EXPLORATION ARCHITECTURE
}

\author{
A Dissertation \\ Presented to \\ The Academic Faculty
}

By

David A. Young

\begin{abstract}
In Partial Fulfillment
of the Requirements for the Degree of

Doctor of Philosophy in the

School of Aerospace Engineering
\end{abstract}

Georgia Institute of Technology

May 2007

Copyright (C) 2007 by David A. Young 


\section{AN INNOVATIVE METHODOLOGY FOR ALLOCATING RELIABILITY AND COST IN A LUNAR EXPLORATION ARCHITECTURE}

Approved by:

Dr. Alan Wilhite, Chairman School of Aerospace Engineering Georgia Institute of Technology

Dr. Vitali Volovoi

School of Aerospace Engineering Georgia Institute of Technology

Dr. Joseph Saleh

School of Aerospace Engineering Georgia Institute of Technology
Dr. Mitchell L. R. Walker II School of Aerospace Engineering Georgia Institute of Technology

Dr. Stylianos Kavadias School of Business Georgia Institute of Technology

Mr. Daniel O’Neil

Marshall Space Flight Center National Aeronautics and Space Administration

Date Approved: March 30, 2007 


\section{ACKNOWLEDGEMENTS}

I would like to thank everyone who has helped me throughout my trek through this dissertation process. I would like to thank my thesis advisor, Dr. Alan Wilhite, for his guidance through this research project. I would also like to express gratitude to the other members of my thesis committee, Dr. Stylianos Kavadias, Mr. Daniel O’Neil, Dr. Joseph Saleh, Dr. Vitali Volovoi, and Dr. Mitchell Walker. Each committee member has devoted his time and insight into this project and helped guide this research to its completion. I would also like to thank Dr. John Olds, who helped me immensely my first three years at Georgia Tech, and whose research interests directed me into the conceptual design field.

I would also like to thank the members of the Space System Design Laboratory at Georgia Tech. This research would not have been possible without the help and distractions provided by my friends at SSDL. I would especially like to thank my officemate, Zack Krevor, for providing a willing ear to my research questions.

Finally, I would like to thank my family for their support through this research process. Mom and Dad, you instilled me with the values and work ethic that made this research possible. I especially want to thank my wife, Kimberly. Kimberly, you provided me with the love and support necessary to complete such an arduous process as this Ph.D. dissertation. Without you this would not have been possible. Therefore I dedicate this thesis to my wife, my love, Kimberly. 


\section{TABLE OF CONTENTS}

ACKNOWLEDGEMENTS ..........................................................................................III

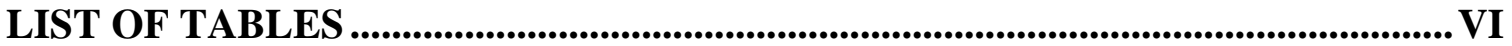

LIST OF FIGURES .......................................................................................................... IX

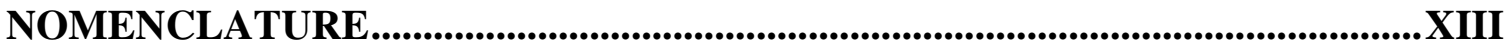

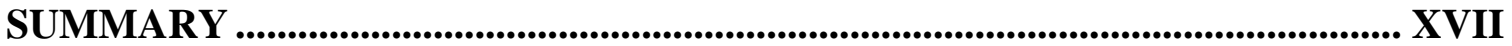

CHAPTER 1 INTRODUCTION .................................................................... 1

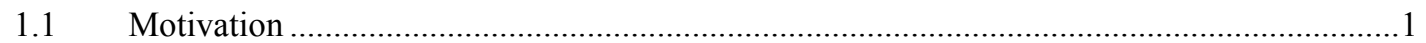

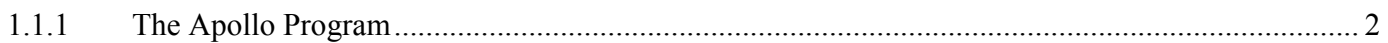

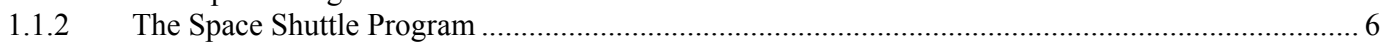

Exploration Systems Architecture Study ........................................................................... 10

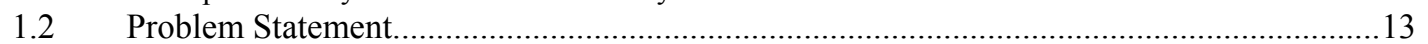

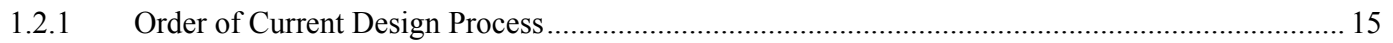

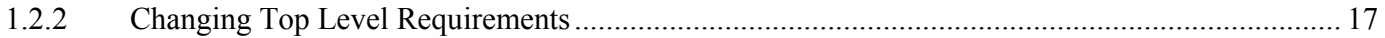

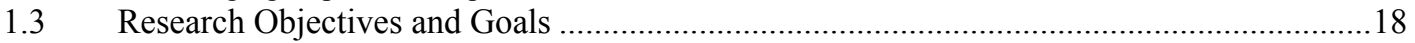

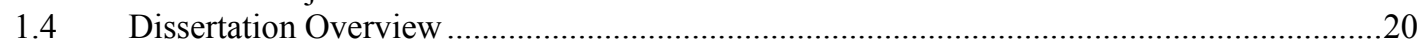

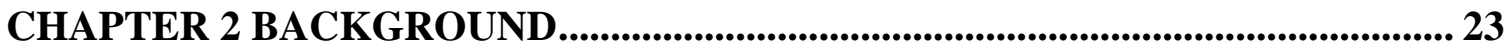

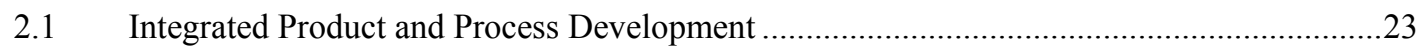

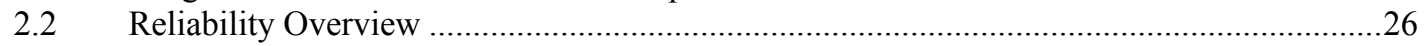

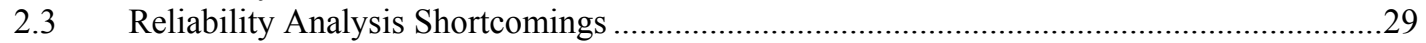

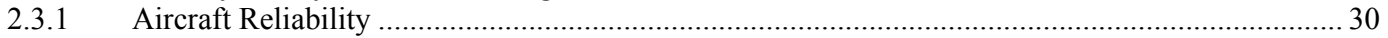

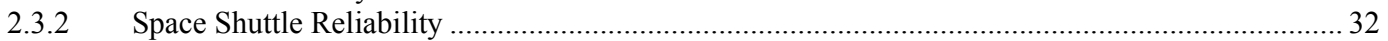

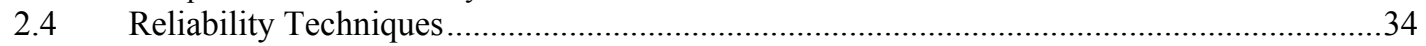

2.4.1 Failure Mode Effects and Criticality Analysis..................................................................... 35

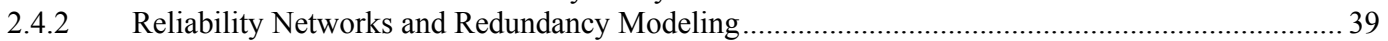

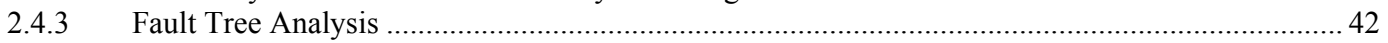

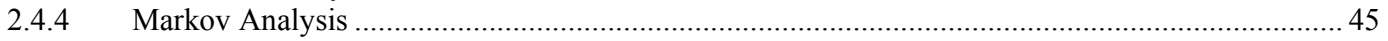

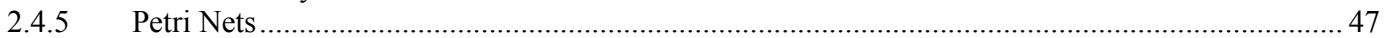

2.4.6 Probabilistic Risk Assessment.......................................................................................... 49

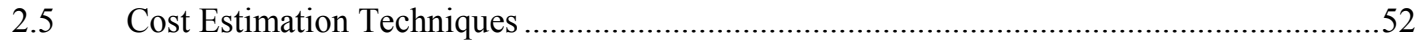

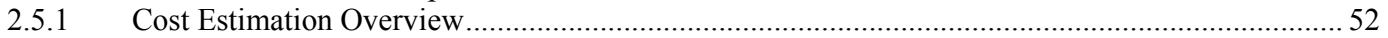

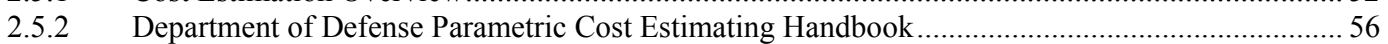

2.5.3 NASA Air Force Cost Model (NAFCOM) ...................................................................... 58

2.5.4 Transcost Cost Estimating Handbook ........................................................................ 58

$2.6 \quad$ Current Conceptual Vehicle Design Process .......................................................60

2.6.1 Conceptual Design Disciplines and the Design Structure Matrix ..............................................60

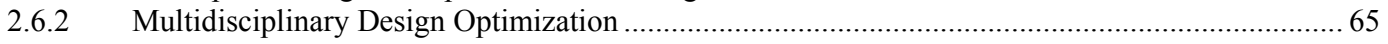

2.6.3 Multidisciplinary Design Optimization With Genetic Algorithms ............................................6. 67

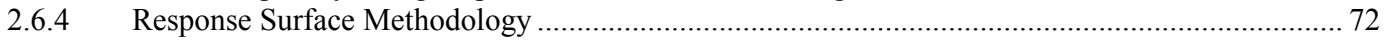

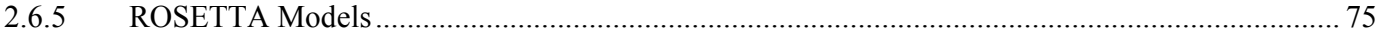

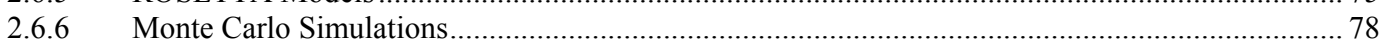

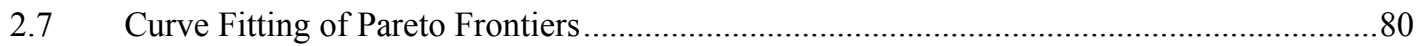

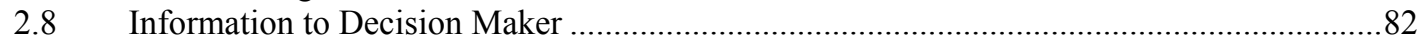

\section{CHAPTER 3 LUNAR ARCHITECTURE MODE SELECTION METHODOLOGY}


3.1 Performance Estimation Using ROSETTA Models and Response Surface Methodology....85

3.1.1 Case Study-Lunar Module ROSETTA Model............................................................................. 85

3.2 Reliability Calculations Using A Dynamically Changing Fault Tree..............................91

3.2.1 General Subsystem Reliability Modeling Methods ............................................................... 92

3.2.2 Propulsion System Reliability Modeling ........................................................................ 101

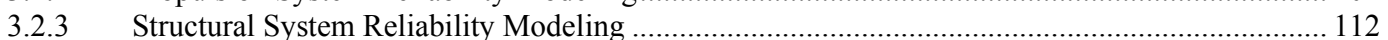

3.2.4 Other Subsystems Modeling Technique ......................................................................... 113

3.2.5 Case Study-Lunar Module ROSETTA Model Reliability Model................................................ 113

3.3 Cost Estimation Using NAFCOM Based Cost Estimating Relationships .........................117

3.3.1 Baseline NAFCOM Cost Estimating Relationships For Lunar Architectures ........................... 117

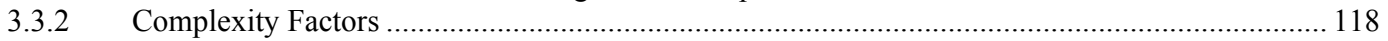

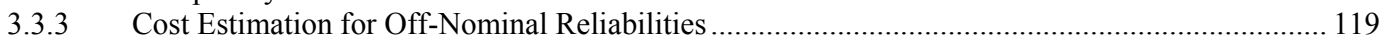

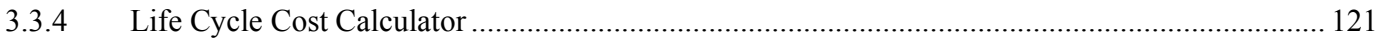

3.3.5 Case Study-Lunar Module ROSETTA Model Costing Model ................................................... 123

3.4 Implementation of ROSETTA Models In A Lunar Architecture Selection Tool .................125

3.4.1 Optimization of Lunar Vehicles ......................................................................................... 126

3.5 Implementation of Uncertainty In Lunar Architecture Selection Tool...........................131

CHAPTER 4 VALIDATIONS OF LUNAR ARCHITECTURE SIMULATION .. 133

4.1 Apollo Lunar Orbit Rendezvous Baseline ...........................................................134

4.2 Apollo Direct Mission Mode Baseline ............................................................................ 141

4.3 Earth Orbit Rendezvous Mission Mode ............................................................................149

4.4 Apollo Lunar Architecture Decision .............................................................................. 156

Exploration System Architecture Study Baseline ...........................................................158

4.6 Comparison of Exploration System Architecture Study Mission Mode and Apollo LOR

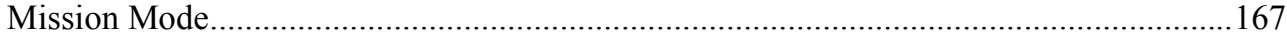

$4.7 \quad$ Validation Conclusions ..................................................................................... 170

CHAPTER 5 APPLICATION OF LUNAR ARCHITECTURE METHODOLOGY FOR DIFFERENT LUNAR MODES ...................................................................... 172

5.1 Creation of Pareto Frontiers for Different Lunar Mission Modes .....................................172

5.2 Lunar Orbit Rendezvous Mission Mode Optimization................................................... 177

5.3 Earth Orbit Rendezvous Mission Mode Optimization.......................................................188

5.4 Direct Mission Mode Optimization ................................................................................. 195

5.5 Summary of Apollo Mission Modes............................................................................203

5.6 Current Design Practices Comparison-Exploration System Architecture Study (EOR-LOR)

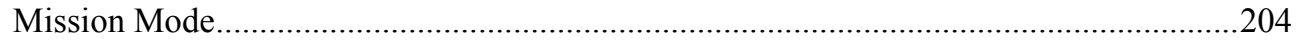

5.7 Summary of ESAS Lunar Architecture Selection ...................................................216

CHAPTER 6 LUNAR ARCHITECTURE MODE SELECTION AND ANALYSIS 219

6.1 Comparison of Apollo Lunar Architecture Selection .....................................................219

6.2 Uncertainty Analysis Applied to Architecture Selection................................................224

6.3 Lunar Mode Selection Via Pareto Frontiers ............................................................230

CHAPTER 7 CONCLUSIONS................................................................................ 231

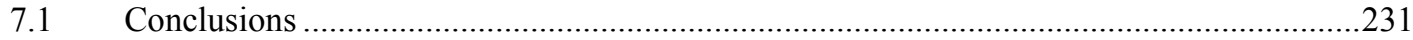

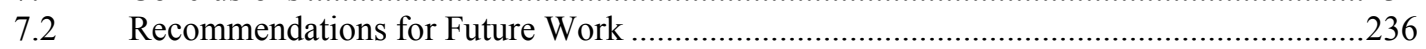

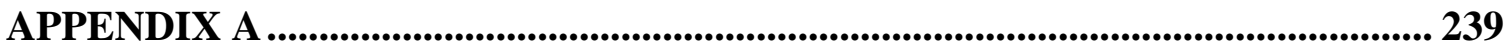

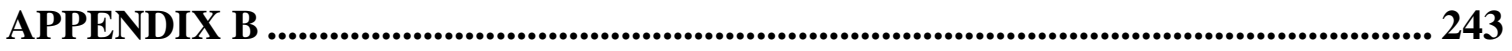

REFERENCES............................................................................................................ 246

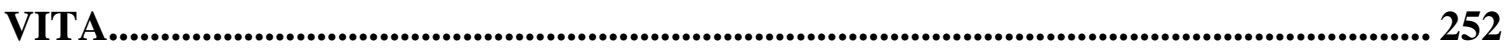




\section{LIST OF TABLES}

Table 1: Shuttle Design Configurations [10] ............................................................ 10

Table 2: Hazard Severity Classification for FHA [17] ................................................. 36

Table 3: Risk Assessment Code Matrix [17] ................................................................. 36

Table 4: Hazard Probability of Occurrence for FHA [17] ............................................. 37

Table 5: Elements in the Space Shuttle Fault Tree [14] ................................................. 44

Table 6: Typical Design Tools for Conceptual Vehicle Design ......................................... 62

Table 7: Characteristics of Design Disciplines for a Conceptual Launch Vehicle [40] .. 66

Table 8: Comparison of MDO Techniques for a Conceptual Launch Vehicle [41]......... 67

Table 9: Burn Summary of Lunar Landers Simulated in ROSETTA Model .................... 87

Table 10: Mission Parameters Ranges for ROSETTA Lunar Module .............................. 87

Table 11: Configuration Inputs for ROSETTA Lunar Module ………………………...... 88

Table 12: Comparison of Different Propellant Types for a Pressure Fed Apollo LM ... 111

Table 13: Comparison of Different Propellant Types for an Expander Cycle Apollo LM 111

Table 14: Complexity Factors for Tank Structural Materials ......................................... 118

Table 15: OEC Baseline Values for Each Lunar Mode ………………......................... 129

Table 16: Propellant Choices for Apollo LOR. …………………………………..... 135

Table 17: Structure Choices for Apollo LOR ………………………………........... 136

Table 18: Comparison of Apollo LOR Performance Results......................................... 138

Table 19: Apollo LOR Design Development Testing and Evaluation Cost Comparison.

Table 20: Apollo LOR Theoretical First Unit Cost Comparison..................................... 140

Table 21: Apollo LOR Loss of Mission Reliability Comparison..................................... 141

Table 22: Propellant Choices for Apollo Direct. ............................................................... 143

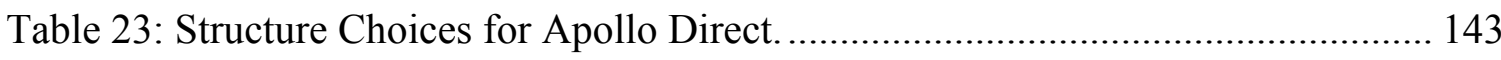

Table 24: Comparison of Apollo Direct to Apollo LOR Performance.............................. 146

Table 25: Apollo LOR and DDT\&E Cost Comparison................................................. 147 
Table 26: Apollo LOR and Direct Theoretical First Unit Cost Comparison.................. 148

Table 27: Comparison of Apollo Direct and Apollo LOR Reliability ........................... 148

Table 28: Propellant Choices for Apollo EOR. ........................................................... 150

Table 29: Structure Choices for Apollo EOR........................................................... 151

Table 30: Comparison of Apollo Mission Modes...................................................... 153

Table 31: Apollo EOR, LOR and Direct Design Development Testing and Evaluation

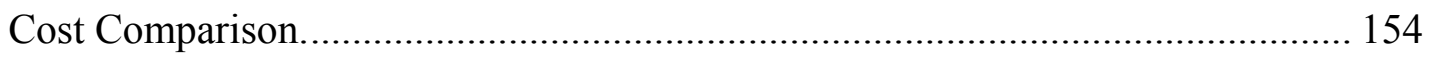

Table 32: Apollo EOR, LOR, and Direct Theoretical First Unit Cost Comparison....... 154

Table 33: Reliability Comparison of Apollo EOR, Direct, and Apollo LOR................ 155

Table 34: Summary of Apollo Lunar Architecture Decision....................................... 157

Table 35: Comparison of Apollo LOR and EOR to ESAS (EOR-LOR) ....................... 159

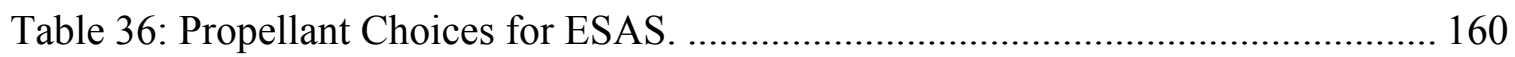

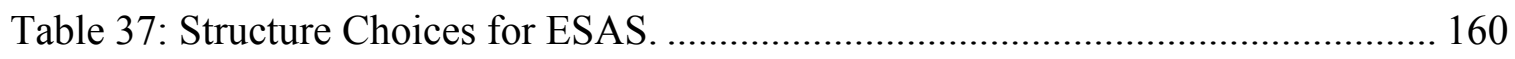

Table 38: Comparison of Apollo LOR Performance Results..................................... 163

Table 39: ESAS Design Development Testing and Evaluation Cost Comparison......... 164

Table 40: ESAS Theoretical First Unit Cost Comparison........................................... 164

Table 41: Cost per Man Hour on the Moon ............................................................. 165

Table 42: Apollo LOR Loss of Mission Reliability Comparison.................................. 166

Table 43: Comparison of ESAS and Apollo LOR Performance Results for a Three Crew

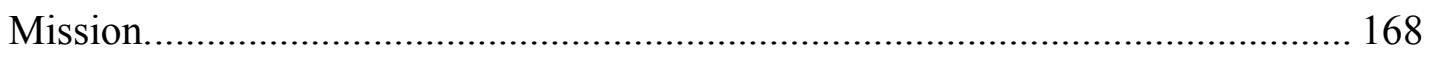

Table 44: Comparison of ESAS and Apollo LOR Design, Development, Testing and Evaluation Results for a Three Crew Mission.................................................... 169

Table 45: Comparison of ESAS and Apollo LOR Theoretical First Unit Results for a

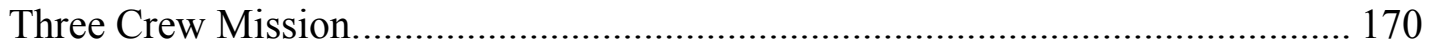

Table 46: Architecture Inputs for the Apollo Lunar Architecture modes..................... 174

Table 47: Morphological Matrix for Command Module ROSETTA Model ................. 175

Table 48: Morphological Matrix for Lunar Module ROSETTA Model........................ 175

Table 49: Morphological Matrix for Service Module ROSETTA Model ..................... 176

Table 50: Morphological Matrix for Launch Vehicle ROSETTA Model ..................... 176

Table 51: Gear Ratio for Apollo LOR Architecture Elements. .................................... 181

Table 52: GA Input Differences Between Apollo Baseline and Pareto Frontier............ 184 
Table 53: GA Input Differences Between Apollo EOR Baseline and Pareto Frontier... 192

Table 54: GA Input Differences Between Apollo Direct Baseline and Pareto Frontier. 200

Table 55: Differences Between Pareto Frontiers and Apollo Baselines........................ 204

Table 56: Architecture Inputs for the ESAS Lunar Architecture ................................. 205

Table 57: Morphological Matrix for Crew Launch Vehicle ROSETTA Model ............ 206

Table 58: Morphological Matrix for Cargo Launch Vehicle ROSETTA Model .......... 206

Table 59: GA Input Differences Between ESAS Baseline and Pareto Frontier ............. 213

Table 60: Pareto Frontier and ESAS Baselines Comparison....................................... 217

Table 61: Pareto Frontier and ESAS Baselines Failure Rate Comparisons................... 217

Table 62: Comparison of Crossover Points for Apollo LOR and Direct Mission Modes

Table 63: Differences Pareto Frontiers and Baselines .................................................. 234

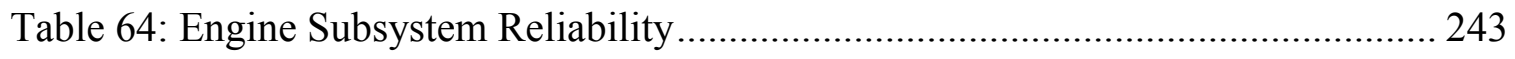

Table 65: Launch Vehicle Tank Subsystem Reliability ............................................ 243

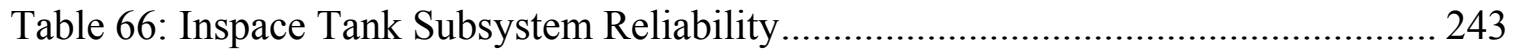

Table 67: Inspace Structure Subsystem Reliability (SM \& CM) ............................... 244

Table 68: Inspace Structure Subsystem Reliability (LM) .......................................... 244

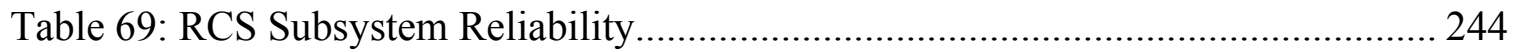

Table 70: Command Module Other Subsystem Reliability Baseline [69]. ................... 244

Table 71: Service Module Other Subsystem Reliability Baseline [69] ........................ 244

Table 72: Lunar Module Other Subsystem Reliability Baseline [69].......................... 245

Table 73: Saturn V Other Subsystem Reliability Baseline [69] ................................. 245 


\section{LIST OF FIGURES}

Figure 1: Apollo Architecture Components [8] ........................................................... 5

Figure 2: Lockheed Star Clipper and Martin Marietta Titan II-M [10] ........................... 8

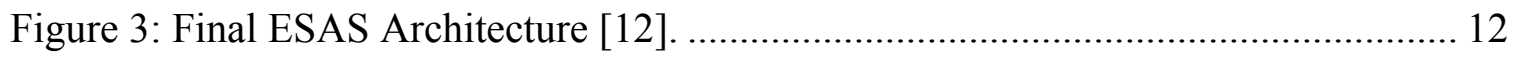

Figure 4: NASA budget as a percentage of the Federal Budget [14] ............................ 14

Figure 5: Design Structure Matrix for Typical Launch Vehicle Design ......................... 16

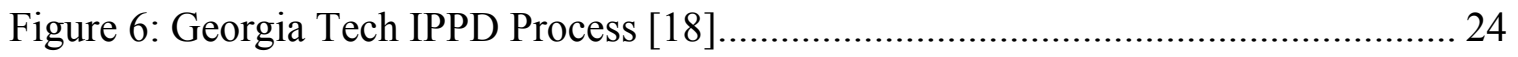

Figure 7: Paradigm Shift in Complex Vehicle Design [19] ........................................... 25

Figure 8: Implementation of the Triplet Definition of Risk used by NASA [23]........... 28

Figure 9: Catastrophic Accident Rate (Hull Loss) of Worldwide Commercial Jet Fleet

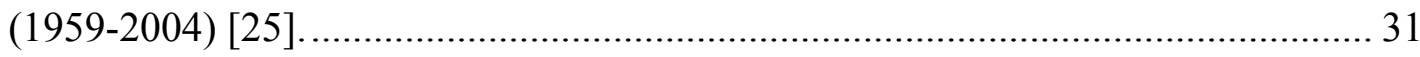

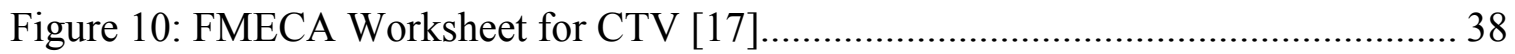

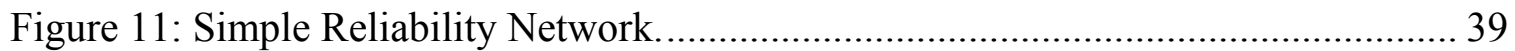

Figure 12: Proposed O-Ring RBD for Space Shuttle [31] ....................................... 41

Figure 13: Fault Tree Analysis for Artemis Conceptual Lunar Lander Design [32]...... 43

Figure 14: Markov Chain of a Parallel System with Two Identical Components [21]... 45

Figure 15: Markov Analysis of a 2 out of 3 System with Warm Spares and Repair [21].47

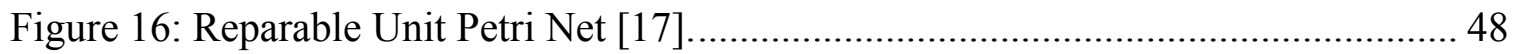

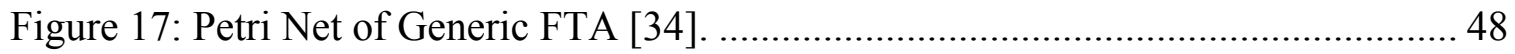

Figure 18: Typical PRA Operational Outline [23] .................................................. 49

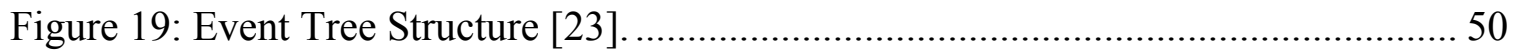

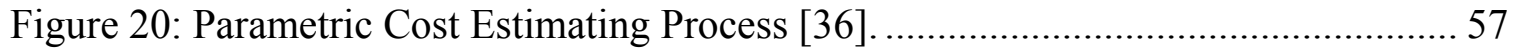

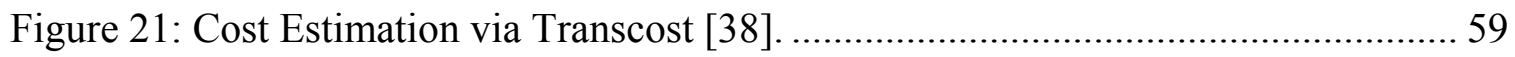

Figure 22: Design Structure Matrix for a Generic Launch Vehicle................................ 61

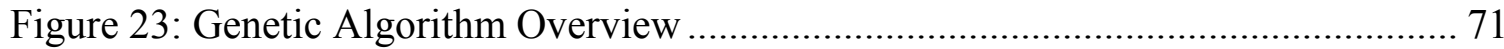

Figure 24: Dimensional Representation of Design of Experiments [44]....................... 73

Figure 25: Results of the Screening Test for a Nozzle [45] ....................................... 74

Figure 26: ROSETTA Models in the ATLAS Framework [47] ................................. 76

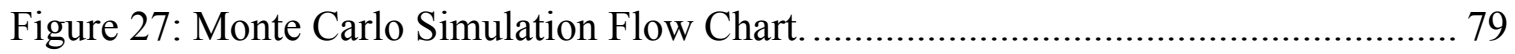




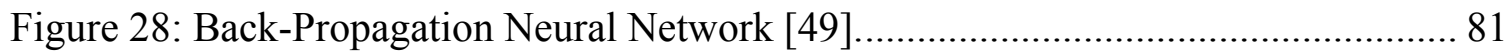

Figure 29: Design Structure Matrix for ROSETTA Lunar Module ................................. 86

Figure 31: Common Cause Failure Implementation into a Single Redundant System. ... 95

Figure 32: Fault Tree Representation of the Propulsion System for a Generic Launch Vehicle. 98

Figure 33: Expander Cycle Engine Schematic [65]................................................. 102

Figure 34: Schematic of Gas Generator Engine Cycle [65] .......................................... 103

Figure 35: Schematic of Staged Combustion Engine Cycle [65] .................................. 104

Figure 36: De-rating Engine Reliability for the Space Shuttle Main Engine. ................ 106

Figure 37: Rubberized Engine Reliability for the SSME and RD-180 based Engines... 108

Figure 38: Engine Demonstrated Reliability as a Function of Testing.......................... 109

Figure 39: Costing K Factor for Engine Testing ....................................................... 110

Figure 40: Fault Tree of Lunar Module ..................................................................... 114

Figure 41: Fault Tree of Lunar Module with Engine Out Capability ............................ 114

Figure 43: Life Cycle Cost Spreading for a Lunar Architecture ………........................ 122

Figure 44: Screenshot of Lunar Module Cost Model ................................................. 124

Figure 45: Monte Carlo Methodology of Lunar Architecture Selection ........................ 126

Figure 46: Monte Carlo Design Space Exploration of Apollo-like Lunar Module ........ 127

Figure 47: Pareto Frontier of Monte Carlo Design Space Exploration of Apollo-like

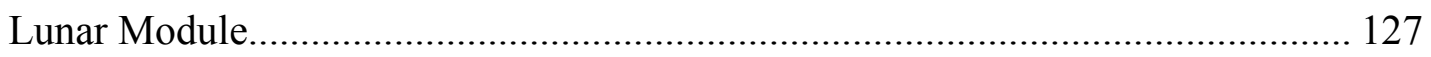

Figure 48: Integrated Lunar Architecture Selection Tool with Genetic Algorithm........ 128

Figure 49: Comparison of Monte Carlo Analysis and Genetic Algorithm for an Apollo-

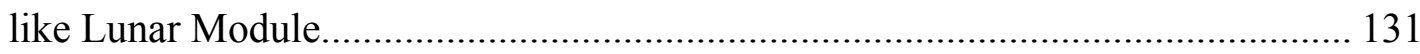

Figure 50: Lunar Orbit Rendezvous Mission Mode [4]................................................ 134

Figure 51: ModelCenter Based Lunar Orbit Rendezvous Simulation [73] ................... 137

Figure 52: Direct Mission Mode [4] ..................................................................... 142

Figure 53: ModelCenter Based Direct Mission Simulation [73] ................................... 145

Figure 54: EOR Mission Mode [4] ..................................................................... 150

Figure 55: ModelCenter Based EOR Mission Simulation [73] ................................... 152

Figure 56: ModelCenter Based ESAS Simulation [73] ............................................... 162

Figure 57: Pareto Frontier of the Apollo LOR Architecture.............................................. 178 
Figure 58: Summary of GA Produced Inputs for Apollo LOR Architecture ................ 178

Figure 59: Redundancy in the Apollo LOR Pareto Frontier...................................... 182

Figure 60: Comparison of Apollo Baseline to Pareto Frontier Optimal Points .............. 183

Figure 61: LOC Pareto Frontier for Apollo LOR ....................................................... 185

Figure 62: Neural Network Curve Fit of Pareto Frontier .......................................... 186

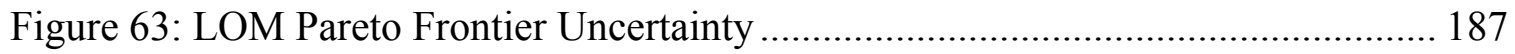

Figure 64: LOC Pareto Frontier Uncertainty ......................................................... 187

Figure 65: Pareto Frontier of the Apollo EOR Architecture........................................ 189

Figure 66: Summary of GA Produced Inputs for Apollo EOR Architecture ................ 189

Figure 67: Comparison of Apollo EOR Baseline to Pareto Frontier Optimal Points ..... 191

Figure 68: LOC Pareto Frontier for Apollo EOR …................................................... 193

Figure 69: EOR Mission LOM Pareto Frontier Uncertainty ....................................... 194

Figure 70: EOR Mission LOC Pareto Frontier Uncertainty ..................................... 195

Figure 71: Pareto Frontier of the Apollo Direct Architecture...................................... 196

Figure 73: Comparison of Apollo Direct Baseline to Pareto Frontier Optimal Points ... 199

Figure 74: LOC Pareto Frontier for Apollo Direct ................................................... 201

Figure 75: Direct Mission LOM Pareto Frontier Uncertainty ...................................... 202

Figure 76: Direct Mission LOC Pareto Frontier Uncertainty ...................................... 203

Figure 77: Pareto Frontier of the ESAS Architecture ............................................. 207

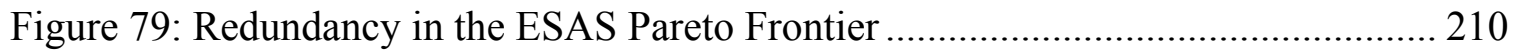

Figure 80: Comparison of ESAS Baseline to Pareto Frontier Optimal Points .............. 212

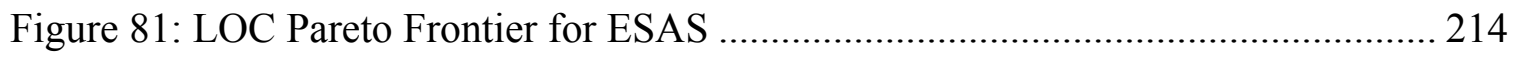

Figure 82: ESAS LOM Pareto Frontier Uncertainty ............................................ 215

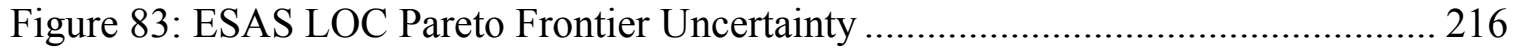

Figure 84: Mission Mode Selection for Apollo Lunar Architectures LOM .................. 220

Figure 85: Zoomed in Comparison of Pareto Frontiers for Apollo LOR and Direct ..... 222

Figure 86: Mission Mode Selection for Apollo Lunar Architectures LOC .................... 223

Figure 87: Best Case LOM Scenario for Apollo Lunar Modes .................................. 225

Figure 88: Worst Case LOM Scenarios for Apollo Mission Modes ............................ 226

Figure 89: Uncertainty Bands for Apollo Mission Mode Selections............................ 228

Figure 90: Comparison of Uncertainty Bands for LOC of Apollo Mission Modes ....... 229 
Figure 91: Integrated Lunar Architecture Selection Tool (Revisited) ......................... 233

Figure 92: Simulation Outputs for Apollo LOR Architecture ...................................... 239

Figure 93: Simulation Outputs for Apollo EOR Architecture ................................... 240

Figure 94: Simulation Outputs for Apollo Direct Architecture ................................... 241

Figure 95: Simulation Outputs for ESAS EOR-LOR Architecture ............................. 242 


\section{NOMENCLATURE}

\begin{tabular}{|c|c|}
\hline $\mathrm{AAO}$ & All-at-Once \\
\hline Al & Aluminum \\
\hline Al-Li & Aluminum-Lithium \\
\hline APAS & Aerodynamic Preliminary Analysis System \\
\hline ATLAS & Advanced Technology and Lifecycle Analysis System \\
\hline BLISS & Bi-Level Integrated System Synthesis \\
\hline CAD & Computer Aided Design \\
\hline CaLV & Cargo Launch Vehicle \\
\hline $\mathrm{CCD}$ & Central Composite Design \\
\hline $\mathrm{CDF}$ & Cumulative Density Function \\
\hline CER & Cost Estimating Relationships \\
\hline CEV & Crew Exploration Vehicle \\
\hline $\mathrm{CH} 4$ & Methane \\
\hline CLV & Crew Launch Vehicle \\
\hline $\mathrm{CM}$ & Command Module \\
\hline $\mathrm{CO}$ & Collaborative Optimization \\
\hline CTV & Crew Transfer Vehicle \\
\hline DDT\&E & Design, Development, Testing, and Evaluation \\
\hline DoD & Department of Defense \\
\hline DOE & Design of Experiments \\
\hline DSM & Design Structure Matrices \\
\hline EDS & Earth Departure Stage \\
\hline
\end{tabular}




$\begin{array}{ll}\text { ESAS } & \text { Exploration Systems Architecture Study } \\ \text { EO } & \text { Engine Out } \\ \text { EOR } & \text { Earth Orbit Rendezvous } \\ \text { ESMD } & \text { Exploration Systems Mission Directorate } \\ \text { ETO } & \text { Earth to Orbit } \\ \text { FHA } & \text { Functional Hazard Analysis } \\ \text { FMECA } & \text { Failure Mode Effects and Criticality Analysis } \\ \text { FTA } & \text { Fault Tree Analysis } \\ \text { GR } & \text { Gear Ratio } \\ \text { Gr-Ep } & \text { Graphite Epoxy } \\ \text { IOC } & \text { Initial Operating Capability } \\ \text { IPPD } & \text { Integrated Product and Process Development } \\ \text { IPT } & \text { Integrated Product Team } \\ \text { Isp } & \text { Specific Impulse } \\ \text { ISS } & \text { International Space Station } \\ \text { LBM } & \text { Lunar Braking Module } \\ \text { LEM } & \text { Lunar Excursion Module } \\ \text { LH2 } & \text { Liquid Hydrogen } \\ \text { LLO } & \text { Low Lunar Orbit } \\ \text { LM } & \text { Lunar Module } \\ \text { LOC } & \text { Loss of Crew } \\ \text { LOR } & \text { Lunar Orbit Rendezvous } \\ \text { LOV } & \text { LOX }\end{array}$




\begin{tabular}{|c|c|}
\hline LSAM & Lunar Surface Access Module \\
\hline MER & Mass Estimating Relationship \\
\hline MDO & Multidisciplinary Design Optimization \\
\hline MLP & Multilayer Perceptron \\
\hline MMC & Metal Matrix Composites \\
\hline N204 & Nitrogen Tetroxide \\
\hline NAFCOM & NASA Air Force Cost Model \\
\hline NASA & National Aeronautical and Space Administration \\
\hline NN & Neural Network \\
\hline NPD & New Product Development \\
\hline OMB & Office of Management and Budget \\
\hline $\mathrm{PDF}$ & Probability Density Function \\
\hline PRA & Probabilistic Risk Assessment \\
\hline RAC & Risk Assessment Code \\
\hline RBD & Reliability Block Diagrams \\
\hline ROSETTA & $\begin{array}{l}\text { Reduced Order Simulation for Evaluating Technologies and } \\
\text { Transportation Architecture }\end{array}$ \\
\hline RP1 & Refined Petrolium-1 \\
\hline RSE & Response Surface Equation \\
\hline RSM & Response Surface Methodology \\
\hline SAIC & Science Applications International Corporation \\
\hline SED & Specific Energy Density \\
\hline SM & Service Module \\
\hline SSDL & Space Systems Design Laboratory \\
\hline STS & Space Transportation System \\
\hline TAOS & Thrust Assisted Orbiter Shuttle \\
\hline
\end{tabular}


TFU

$\mathrm{Ti}$

TPS

UDMH

US

USSR

VSE

WBS
Theoretical First Unit

Titanium

Thermal Protection System

Unsymmetrical Dimethylhydrazine

United States

United Soviet Socialist Republic

Vision for Space Exploration

Work Breakdown Structure 


\section{SUMMARY}

In January 2005, President Bush announced the Vision for Space Exploration. This vision involved a progressive expansion of human capabilities beyond Low Earth Orbit beginning with a return to the moon starting no later than 2020. Current design processes utilized to meet this vision employ performance based trade studies to determine the lowest cost, highest reliability solution. In these design processes, designers trade independent performance variables and then calculate the design discriminators, reliability and costs, of the different architectures. The methodology implemented in this dissertation focuses on a concurrent evaluation of the performance, cost, and reliabilities of lunar architectures. This process directly addresses the top level requirements early in the design process and allows the decision maker to evaluate the highest reliability, lowest cost lunar architectures without being distracted by the performance details of the architecture.

There is a significant amount of research in the operations management community about the need for the decision maker to be provided with not only the optimal solution for a set of requirements, but also the set of near-optimal solutions for changing top-level requirements. The methodology presented in this thesis increases the transparency of the process by providing the decision maker with the entire set of solutions in the form of a Pareto frontier. This frontier allows the decision maker to choose the highest reliability solution which meets the budgetary constraints of project 
requirements. This selection from a series of solutions allows the decision maker to be invested in the solution to the architecture while avoiding non-optimal configurations.

To achieve this methodology of bringing optimal cost and reliability solutions to the decision maker, parametric performance, cost, and reliability models are created to model each vehicle element. These models were combined using multidisciplinary optimization techniques and response surface equations to create parametric vehicle models which quickly evaluate the performance, reliability, and cost of the vehicles. These parametric models, known as ROSETTA models, combined with a life cycle cost calculator provide the tools necessary to create a lunar architecture simulation. The integration of the tools into an integrated framework that can quickly and accurately evaluate the lunar architectures is presented. This lunar architecture selection tool is verified and validated against the Apollo and ESAS lunar architectures. The results of this lunar architecture selection tool are then combined into a Pareto frontier to guide the decision maker to producing the highest reliability architecture for a given life cycle cost.

The advantages of this method over traditional design processes are numerous. With this presented methodology, the decision maker can transparently choose a lunar architecture solution based upon the high level design discriminators. This method can achieve significant reductions in life cycle costs (over 40\%) keeping the same architecture reliability as a traditional design process point solution. This methodology also allows the decision maker to choose a solution which achieves a significant reduction in failure rate (over 50\%) while maintaining the same life cycle costs as the point solution of a traditional design process. 


\section{CHAPTER 1}

\section{INTRODUCTION}

The purpose of this dissertation is to improve on the design practices currently employed by the aerospace community. Currently top level design discriminators, such as cost and reliability, are calculated after the vehicle configurations are set. These discriminators are then used to select the winning design among the different candidate designs. This research proposes a methodology to pull these important design discriminators to the front of the design process by combining physics-based parametric models with multidisciplinary optimization techniques and Pareto frontiers to visualize the ideal reliability and cost architecture solutions. Once this ability is verified with historical lunar architectures, the process will be applied to a current lunar architecture selection problem.

\subsection{Motivation}

Man has always been captivated by the notion of exploration. Whether that exploration is being driven by national competition, economic considerations, or scientific motivation the end result has been the expansion of human knowledge. Great explorers have opened the door for future generations to follow in their footsteps. Christopher Columbus was one of the first such explorers. His journey to the Americas in the fifteenth century opened the door for future exploration, settlement, and technological advancement of the western hemisphere. This spirit of exploration 
continued throughout the sixteenth and seventeenth centuries with the expansion of European colonialism in Africa and Asia. In the eighteenth and nineteenth centuries exploration was not only conducted for the gain of national prestige but also for scientific advancement. Darwin's theories of evolution and natural selection were conducted on the H.M.S. Beagle during the 1830s and later in the 1870s when the H.M.S. Challenger conducted the first global marine research expedition [1]. With the exploration of the North and South Poles in the early twentieth century, the age of terrestrial exploration of unknown lands ended, though the people's captivation with exploration had not diminished.

In October 1957, the Soviet Union officially opened a new region of exploration with the launch of Sputnik I. Sputnik I was the world's first artificial satellite. Sputnik I, much like the mission of Columbus five hundred years before, was the opening event in a new age of exploration. Columbus's mission started the European exploration of the western hemisphere; Sputnik marked the start of the space age and the U.S.-U.S.S.R. space race [2]. This race inspired the most ambitious undertaking in human history: landing on the lunar surface.

\subsubsection{The Apollo Program}

The Apollo missions and subsequent lunar landings were all inspired by the same motivation as Columbus and Magellan: international competition and national prestige. It was national prestige not scientific motivation that caused then President John F. Kennedy to proclaim, "Our nation should commit itself to achieving the goal, before this decade is out, of landing a man on the moon and returning him safely to the Earth [3]." This presidential direction and subsequent funding from Congress formed a "crash" 
research program to send man to the moon. This "crash" program allowed NASA to concern itself primarily with returning to the moon by the end of the decade. The top level system metrics for this program was performance (send a man to the moon), schedule (by the end of the decade), and safety. The finances associated with achieving this goal was an afterthought.

When President Kennedy announced that the United States would be pursuing a lunar program there were hundreds of different lunar architectures to choose from. In fact, lunar exploration architectures have been considered for decades. In 1865 Jules Verne wrote a story in which explorers traveled to the moon via a giant cannon. From the Earth to the Moon is one of the earliest ventures of science fiction, and although infeasible, it was one of the first writings on the subject of lunar exploration. In 1961, the National Aeronautics and Space Administration (NASA) was tasked with finding a feasible architecture that would accomplish a safe lunar landing before the decade was over.

The lunar architectures proposed by NASA were quickly reduced to four different modes: the Earth Orbit Rendezvous (EOR), the Lunar Orbit Rendezvous (LOR) and C-5 direct, and the Nova direct [4]. These modes were then compared and contrasted with the existing hardware programs in an attempt to produce the most feasible lunar architecture to safely put a man on the moon before the end of the decade. This was a very manual process with each component of each mode being designed separately and then iterated until a closed solution was achieved. The different lunar modes were calculated with different top level design criteria (payload capability, number of men to the lunar surface, etc.). This resulted in a process which never really compared the modes on an equal basis 
and resulted in over a million man years committed before an architecture was selected [5]. Each mode was championed by a different group at NASA, and the competition was both for the design as well as political ambitions of the lobbying group [6]. After a significant amount of debate the Lunar Orbit Rendezvous was adopted as the mode of choice to explore the moon. Famed rocket designer, Werner Von Braun, attributed the selection of LOR to three factors [7]:

1. LOR appeared most likely to be accomplished within the decade

2. LOR offered adequate performance margins

3. Designs of a reentry vehicle and a lunar landing vehicle constituted the two most critical tasks in producing a lunar spacecraft and separating these two functions into two separate elements was bound to greatly simplify the development of the spacecraft system.

Figure 1 shows the resulting Apollo architecture in the launch configuration. The architecture consists of the Saturn V three stage launch vehicle, a three man Command Module (CM), a Service Module (SM), and a two stage Lunar Excursion Module (LEM). 


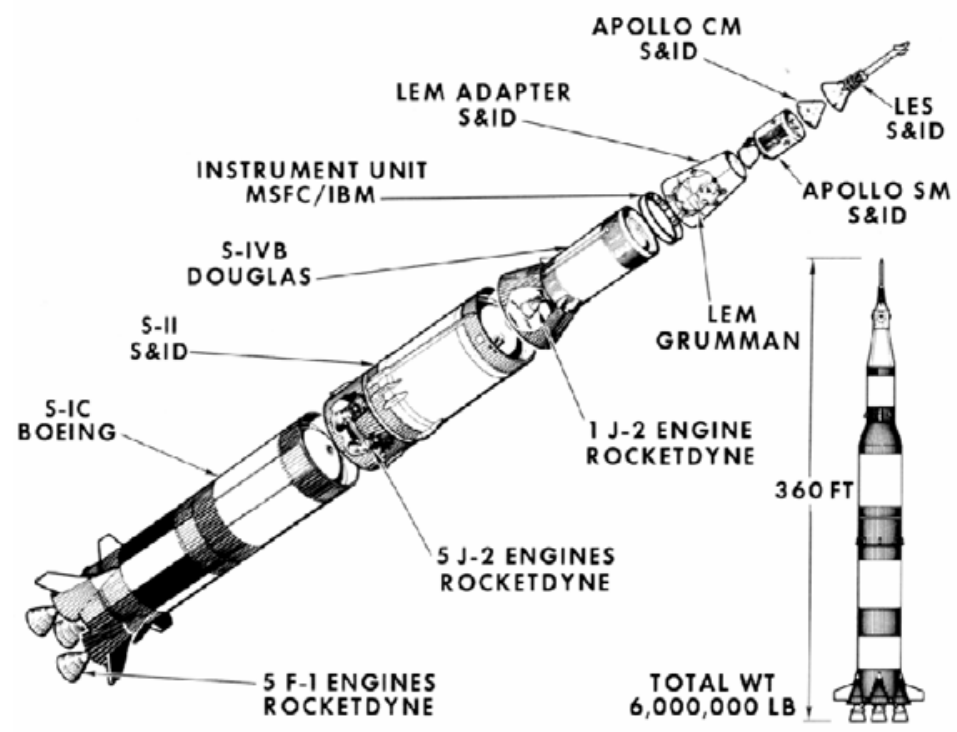

Figure 1: Apollo Architecture Components [8].

Once the architecture was selected, the detailed design analysis was conducted and the architecture tested on a series of flight tests. The space race culminated on July 20, 1969 with the first lunar landing of Neil Armstrong and Edwin Aldrin on Apollo 11. This event signified that the race to the moon was complete, and as with any race the winner was determined. Although NASA and the United States had won the race to the moon, they had lost the motivation that had sustained their effort. Much like the European exploration of the nineteenth century, when the race to discover new territory came to a close, the justification for continued exploration became focused on scientific research. The next six Apollo missions, consisting of five lunar landings, were based on this motivation of scientific research and the desire to gain a better understanding of the origin of Earth. Unfortunately this goal was not nearly as captivating as that of international competition, and the cost, $\$ 2.5$ Billion FY72, was prohibitive given the ongoing Vietnam War [1]. The political and social environment of the 1970s deemed the 
Apollo program unsustainable and it was therefore was terminated with the last lunar mission of Apollo 17 in 1972.

The resulting Apollo program cost over $\$ 25.4$ billion, with only the building of the Panama Canal rivaling the Apollo program's size as the largest non-military technological endeavor ever undertaken by the United States [9]. James E. Webb, the NASA Administrator at the height of the program between 1961 and 1968, always contended that Apollo was a management exercise more than anything else and that the technological challenge, while sophisticated and impressive, was largely within reach at the time of the 1961 decision [9]. This huge undertaking revolutionized systems engineering and design practices in the 1960's. No longer could vehicle designers work independently of the systems within which their designs were required to work. Unfortunately, sustainability was never a major contributor in the design process; and, although successful, the lunar program never continued beyond Apollo.

\subsubsection{The Space Shuttle Program}

Once the Apollo program was terminated, NASA, with the interest of the country in mind, looked for the next captivating space initiative. The United States dabbled in space projects such as the Skylab missions and the Apollo-Soyuz test projects, but did not delve into another major space program. Many believed that the next logical step was to proceed past the Moon to Mars. Such exploration would pose a very interesting problem for designers. To launch a spacecraft large enough to travel to Mars would require either the launch of an enormous rocket into space or a space station equipped to assemble the spacecraft in space. As Apollo was placing the first man on the moon, NASA pushed on to develop nuclear propulsion to power the spacecraft, a space station to provide a place 
to stage the rocket, and a space shuttle to provide inexpensive and reliable access to the space station.

As Apollo was landing and NASA was undergoing huge successes, the budget of the Mars program was cut. NASA administrator, Thomas Paine, decided that the Mars program would have to be put on the shelf, but a joint space station-shuttle plan remained feasible. Further budget cuts during the aerospace recession of the early 1970's caused the office of Management and Budget (OMB) to cut NASA funds to the point where even the shuttle-station concept was too expensive to pursue. Through some NASA perseverance and the support of the Air Force (who favored the shuttle as an inexpensive reconnaissance satellite launch vehicle) the shuttle pushed on to the design stages [10].

The design of the Space Transportation System (STS) concept was a completely different challenge than the design of the Apollo architecture. NASA was now operating within a strict budget to which the design philosophies of the agency would have to adapt. Unfortunately, the precedent of designing to meet performance goals had already been set. NASA designed the Space Shuttle with much the same methodology as Apollo. Each contactor would be left to design its own solution to the space access problem, and NASA would then iterate with the designs to produce a less than optimal solution.

The first design concepts for the Space Shuttle were quite varied. They ranged from the Martin Marietta Titan II-M design, which featured a Titan III rocket with a small orbiter on the top of the vehicle, to the Lockheed Star Clipper, which was a lifting body design with expendable propellant tanks. 

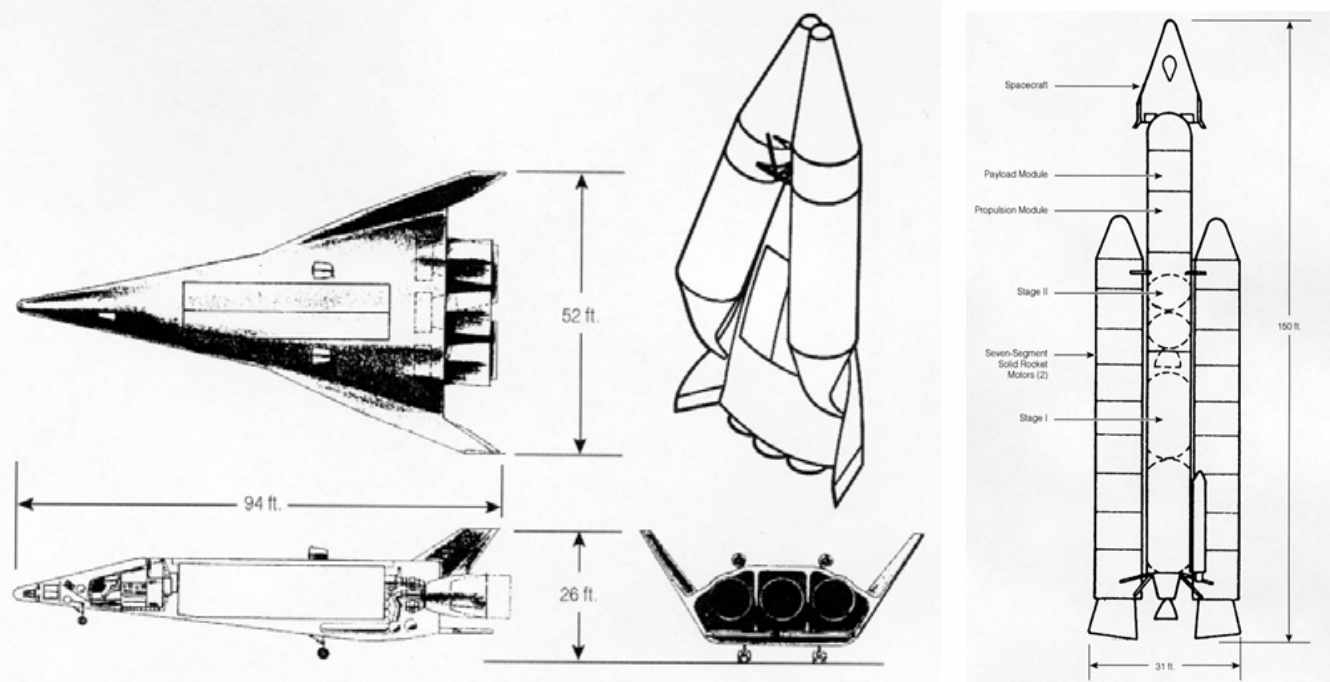

Figure 2: Lockheed Star Clipper and Martin Marietta Titan II-M [10].

Both of these 1967 designs used expendable tanks (as will the shuttle), but at the time a two-stage fully reusable shuttle was favored. It was believed that a totally reusable configuration would, in the end, provide an economic benefit despite the high development costs. In theory, the high launch rate and low cost per flight would overcome the initial design costs.

The debate of what the shuttle should look like continued for several years. Many different launch systems were proposed, all of which were at least partially reusable and most had a cylindrical payload bay of at least 16 by 60 feet to accommodate the needs of NASA's lone advocate: the Air Force. The economic turndown of the seventies dramatically changed NASA's outlook for the Space Shuttle. NASA was forced to justify the benefit of a reusable spacecraft over the existing expendable rockets. The imposed cost ceiling influenced many of the shuttle's design characteristics. First, it was decided that an aluminum frame would be used in place of a titanium structure. The 
titanium would be lighter and require less thermal protection, but the high cost of the metal and the lack of experience building airframes out of titanium forced the aluminum structure on the shuttle designers.

The next cost cutting approach was to return to the early designs of the late sixties and put the fuel in an expendable tank separate from the orbiter. This was advantageous due to the low density of liquid hydrogen. Although light, the hydrogen required a great deal of structure, resulting in a heavy airframe. By placing the hydrogen externally, the airframe would be reduced, resulting in a lighter orbiter.

A third cost cutback was achieved in the selection of a Thrust Assisted Orbiter Shuttle (TAOS). A severe disadvantage to this method of unmanned rocket boosters is that rockets would have to be retrieved manually after falling to earth much like the expendable space capsules of the Apollo missions. The major advantages came in development costs, which were significantly lower for these unmanned boosters. Other alternatives were considered in the TAOS discussion as well. The designers needed to decide whether to use a high-pressure feed liquid system or a solid booster. The solid booster was chosen in the end due to its low development costs, lighter structure, and the fact the shell would fill with air on decent and float for easy recovery.

A final cost cutting measure was initiated by the OMB to decrease the overall size of the shuttle to a $30,000 \mathrm{lb}$ payload with a 10 by 30 foot bay. This would be much less expensive to develop and the shuttle could still carry over $80 \%$ of the intended cargo. NASA resisted the idea, as did the Air Force, which was interested in the large payload bay to launch its own satellites. A cost summary was done for the five competing options for the orbiter. It seemed that due to the high costs of the subsequent options, the first 
option was going to be selected, but NASA and the Air Force argued that the fourth was necessary (Table 1). In early 1972, George Shultz of the OMB formally approved the larger NASA sponsored design somewhat spontaneously and sent the issue to President Nixon for approval.

Table 1: Shuttle Design Configurations [10].

\begin{tabular}{lccccc}
\hline CASE & 1 & 2 & $2 \mathrm{~A}$ & 3 & 4 \\
Payload bay (ft.) & $10 \times 30$ & $12 \times 40$ & $14 \times 45$ & $14 \times 50$ & $15 \times 60$ \\
Payload weight (lbs) & 30,000 & 30,000 & 45,000 & 65,000 & 65,000 \\
Development Cost (\$billions) & 4.7 & 4.9 & 5 & 5.2 & 5.5 \\
Operating Cost (\$millions/flight) & 6.6 & 7 & 7.5 & 7.6 & 7.7 \\
Payload costs (\$/pound) & 220 & 223 & 167 & 115 & 118 \\
\hline
\end{tabular}

This table enumerates the design problems faced during the shuttle design process. As the requirements changed, the design process had to be manually rerun with performance variables as the main variables in the design process. Cost was only evaluated as an afterthought and then used as a discriminator between the designs. This flawed design process resulted in a vehicle which has never reached its cost or reliability goals. As a result the Space Shuttle cost over $\$ 33$ billion dollars FY05 to become operational with an average cost of $\$ 1.3$ Billion FY05 per flight [11].

\subsubsection{EXPLORATION SYSTEMS ARCHITECTURE STUDY}

With the recent Columbia accident the future of the Space Shuttle has been put into question. In January 2004, President George W. Bush announced a new direction for NASA. In his address to the public, the president announced a new Vision for Space Exploration in which NASA would return humans to the Moon by 2020. This new 
direction for NASA is similar to the redirection NASA faced at the beginnings of both the Apollo and Space Shuttle eras. NASA is now facing the task of designing a set of vehicles to return man to the moon and also to continue to service the international space station. Dr. Michael Griffin was named the NASA administrator in April 2005 and further refined the design tasks. Dr. Griffin set out to accelerate the new vehicle design, called the Crew Exploration Vehicle (CEV), to be completed on the eve of the Space Shuttle retirement in 2010. With a new administrator and a new space vision, NASA set out to design a new set of vehicles to return to the moon.

During the summer of 2005 the Exploration Systems Architecture Study (ESAS) team was assembled at NASA headquarters to conduct the design process to create the new lunar architecture to accomplish the Vision for Space Exploration. The team was assembled from 20 core team members from various NASA field centers with over 400 additional staff members assisting the design of the architecture. The ESAS team was constituted to work towards the completion of the following four tasks [12]:

1. Complete assessment of the top-level Crew Exploration Vehicle (CEV) requirements and plans to enable the $\mathrm{CEV}$ to provide crew transport to the ISS; and, to accelerate the development of the CEV and crew-launch system to reduce the gap between the shuttle retirement and CEV Initial Operating Capability (IOC),

2. Define top-level requirements and configurations for crew and cargo launch systems to support the lunar and Mars exploration programs

3. Develop a reference lunar exploration architecture concept to support sustained human and robotic lunar exploration operations and, 
4. Identify key technologies required to enable and significantly enhance these reference exploration systems and a reprioritization of near-term and far-term technology investments.

The ESAS team went about defining the architecture using the traditional design process of determining performance characteristics and then defining costs for each of the contending architecture elements. The best vehicle elements were then chosen based upon both performance and cost. The final ESAS architecture design is given below as Figure 3 .

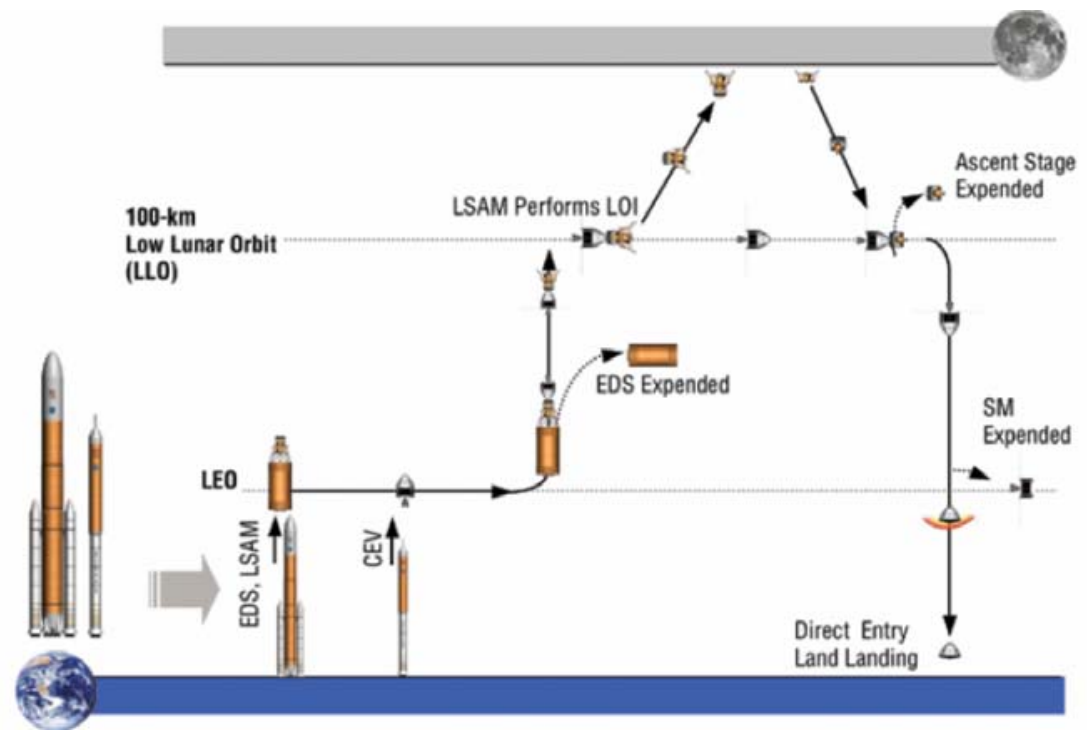

Figure 3: Final ESAS Architecture [12].

The final architecture selection involves shuttle derived launch vehicle elements with a new blunt body capsule CEV. Each element in the ESAS architecture was independently designed and then the reliability and cost were calculated after the design was set. The 
reliability and cost were then used as discriminators in the design process to choose the final architecture. These discriminators are noted in the ESAS final report;

"The (Crew Launch Vehicle, (CLV)) configuration was selected due to its lower cost, higher safety/reliability, its ability to utilize existing human-rated systems and infrastructure and the fact that it gave the most straight forward path to a CaLV [13]"

In fact, the ESAS report claims nine advantages/features of the selected architecture. All nine of the advantages have to do with cost, affordability, or reliability/safety. These claims prove that reliability and cost seem to be the major discriminators used by the ESAS team to select the preferred system. Unfortunately, this methodology of comparing the costs and reliabilities of elements after the design process is both inefficient and slow and does not produce an optimal design. A further problem occurs when the requirements of the project change. In the months preceding this paper, changing cost caps and performance constraints have caused a massive redesign of the ESAS architecture. This has resulted in delays in the selection of the architectures since the design process must be repeated with the new performance criteria and constraints. To date, the NASA Constellation Program and the sponsoring Exploration Systems Mission Directorate (ESMD) continues to study lunar exploration architectures.

\subsection{Problem Statement}

Complex space vehicle designs like that of the STS and Apollo missions require hundreds of thousands of hours of conceptual design work to obtain the best 
configuration. The criteria used to determine the best configuration have historically been linked to performance variables such as speed and range for aircraft, or payload capacity for a launch vehicle. During the 1980s and 1990s there was a paradigm shift in the criteria used to select vehicles. Aircraft and spacecraft were no longer selected based upon the typical performance discriminators, but now cost and reliability were of increased importance to the customer. As the NASA budget began to shrink in the postApollo era the use of cost as a discriminator has become a priority.

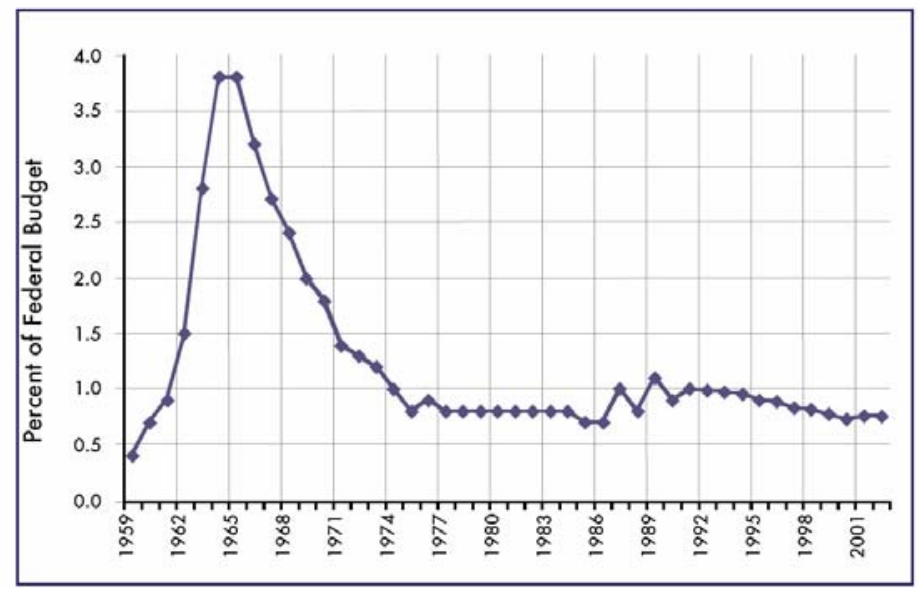

Figure 4: NASA budget as a percentage of the Federal Budget [14].

This cost consciousness directly affected the design of the STS and evidenced continued impact throughout the life of the shuttle. In fact, the Columbia Accident Review Board attributes some of the STS programs to the diminishing budget;

"The White House, Congress, and NASA leadership exerted constant pressure to reduce or at least freeze operating costs. As a result, there was little margin in the 
budget to deal with the unexpected technical problems or make Shuttle improvements.[14]”

NASA's focus shifted significantly after the Columbia disaster of 2003. Since then, NASA has instituted a safety-first policy promoting reliability and safety to the forefront of vehicle design. This focus has continued through the ESAS design process. As the ESAS final report noted;

"This approach (ESAS) resulted in an architecture that met vehicle and mission requirements for cost and performance, while ensuring that the risks to the mission and crew were acceptable [13]."

This quote demonstrates the importance of analyzing reliability while addressing cost and performance. As the top level programmatic requirements change, there is no currently used process to automatically change the architecture element's designs and resulting reliability.

\subsubsection{Order of CURRENT DESIGN Process}

Current design processes rely on the use of legacy tools to evaluate the performance parameters of a design. Cost and reliability of the closed vehicle design are then calculated. The reliability and costs are used as the major discriminators between different vehicle solutions for architecture elements. A primary flaw with this process lies in the complexity of the space vehicle problem, which requires designers to restrict the design before complete information about the requirements is obtained. A second 
concern is the solutions to the design process tend to produce optimal cost or reliability configurations indirectly by comparing the results of the investigated vehicles. This current design process is shown in the design structure matrix for a typical space launch vehicle.

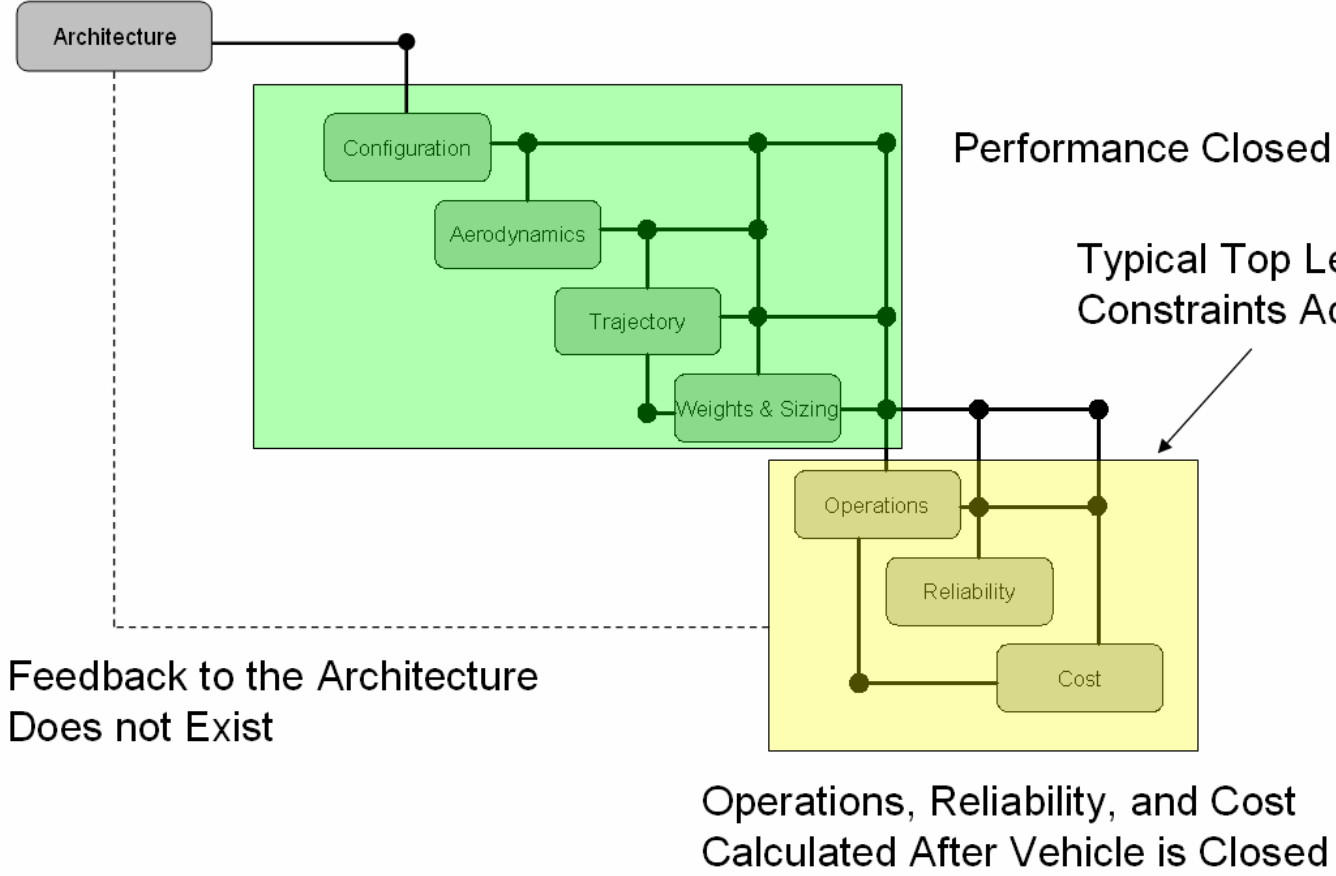

Figure 5: Design Structure Matrix for Typical Launch Vehicle Design

As Figure 5 indicates, the design process for a vehicle is completed in two stages. The first iteration loop is highlighted in green. This loop closes the performance of the vehicle and includes all of the engineering analysis necessary to design a launch vehicle. Once the performance is closed, the typical design discriminators such as cost and reliability are calculated. Unfortunately, this method is cumbersome and requires the 
design process to be completed for each vehicle before the major design discriminators can be calculated and compared.

A solution to this problem of treating cost and reliability as an afterthought is to present the decision maker with the ideal solutions for varying design budgets. This will be accomplished by creating a Pareto frontier of the reliability and cost design space so that the decision maker can seamlessly see the effect of changing budgets on the reliability of the system. This method will further allow the decision maker to see the top level criteria (reliability and cost) without becoming bogged down in the design decisions for the different vehicles in the architecture.

\subsubsection{ChANGing ToP LEVEL REQUIREMENTS}

A further problem occurs when the requirements of the project change. In fact, requirement instability is one of the main issues facing designers today [15]. Meeting changing requirements is especially difficult within a highly complex system of systems, like a lunar architecture. As requirements change, major redesigns are necessary to meet the new and often uncompromising goals of the customer. This is especially true of the budget of the project. As demonstrated by the STS program in section 1.1, when the budget of the shuttle was changed, a complete redesign of the vehicle became necessary. The shuttle program went through numerous redesigns based upon the changing budget of the shuttle system (Table 1). Currently, there is no methodology that allows the decision makers to switch between architecture elements or vehicle design options as the top level requirements change. The design process must evolve to allow changing requirements to quickly filter through the design process, as the instability of requirements is likely to continue to be a problem. 
A solution to the problem of changing requirements is accomplished by creating an integrated framework to solve lunar architectures quickly. With this integrated framework a Pareto frontier can be created for the varying requirements to accurately compare the different requirement changes to find the highest reliability configuration for a given design budget.

\subsection{RESEARCH OBJECTIVES AND GOALS}

The main objective of this research is to create a methodology to treat investment as an independent variable in the design process with respect to reliability. This will allow the top level customer requirements such as budget and required reliability to be directly addressed by the vehicle designers. Also, if cost is included as an independent variable in a design process, it can be easily traded. This method will allow the maximum reliability of a system to be achieved for a given design budget. To accomplish this objective the reliability of different classes of vehicles must be calculated as a function of the life cycle costs.

\section{- Goal 1: Create a methodology to bring design discriminators such as cost and reliability to the forefront of the design process.}

To accomplish this goal, a survey of existing design techniques was conducted. These design techniques are then evaluated and the pertinent parts of the processes are combined to create a new design philosophy. This philosophy will need to present the decision maker with ideal reliability for the changing cost requirements of a lunar architecture. 
- Goal 2: Create and validate a tool that can quickly and efficiently evaluate lunar architectures to provide accurate reliability and cost information to the decision maker.

This goal is necessary to prove that the method defined in Goal 1 is accurate and applicable to today's lunar design problems. The method will be validated against historical lunar architectures (Apollo) as well as current design studies (ESAS). The performance of the architecture tool will be gauged and the accuracy of the answers, performance, cost, and reliability, will be compared with results obtained from previous design methods.

\section{- Goal 3: Apply the tool to existing lunar architecture modes so that the optimal reliability lunar mode can be evaluated for changing life cycle costs.}

Once the method and the lunar architecture tool are validated in Goal 2, the lunar architecture selection tool will be used to reanalyze the Apollo lunar architecture mode decision [7]. The given methodology will be applied to each of the lunar mission modes under consideration in the 1960s. The Pareto frontiers of the optimal solutions for each of the mission modes will be compared with the solutions obtained during the actual Apollo mission mode selection process. The optimal Pareto frontier will then be created and the optimal solution for any given Apollo budget can be easily obtained. Once the Apollo decision is reanalyzed, the procedure will be applied to the ESAS architecture 
selected by the ESAS team. Due to constraints on the project, the mission mode will be kept constant; however, the architecture elements will be allowed to change to produce the optimal vehicle configurations for the mission mode.

\subsection{Dissertation OVERVIEW}

This dissertation will cover multiple aspects of the design problem faced by today's decision makers. The first chapter discussed the history of relevant major NASA design projects. This history provided a motivation for the necessity of a new design process and gave an outline of the proposed research objectives. Chapter 2 is a background of the relevant literature pertaining to this research. This literature review includes a summary of current reliability practices and their shortcomings. This summary covers the current techniques used in reliability modeling including; Failure Mode Effects and Criticality Analysis, Reliability Block Diagrams, Redundancy Modeling, Markov Analysis, Fault Tree Analysis, and Probabilistic Risk Assessment. This review of reliability techniques will pave a way to create the dynamic fault tree analysis which will be used to create the optimal Pareto Frontiers for the lunar architectures.

Chapter 2 continues with a review of current costing techniques. These techniques include weight based Cost Estimating Relationships (CERs), as well as the NASA Air Force Cost Model (NAFCOM). The shortcomings in these costing methods will be addressed and their application to the methodology of this research will be demonstrated. The review of literature will continue with a complete analysis of current conceptual vehicle design practices. This will include topics such as Design Structure Matrices, Multidisciplinary Design Optimization, Response Surface Methodology, the creation of Reduced Order Simulation for Evaluating Technologies and Transportation 
Architecture (ROSETTA) models, as well as the Monte Carlo Simulations which are used to complete an uncertainty analysis.

Chapter 3 discusses the approach used to implement the methodology as mentioned in the research objectives. This methodology involves the creation of ROSETTA models that can accurately describe the performance, cost, and reliability of different architecture elements (vehicles) efficiently and robustly. These ROSETTA models contain Response Surface Equations to model high fidelity physics based analyses. The ROSETTA models also contain a NAFCOM based cost estimating relationships, and a dynamically changing fault tree analysis to build up the reliability of the element. These ROSETTA models will automatically calculate the cost, reliability, safety, and performance as the configuration of the vehicle changes. ROSETTA models will then be combined in an integrated design framework to accurately and quickly evaluate a lunar architecture. This lunar architecture tool will then be mated with a Genetic Algorithm optimizer to produce the optimal reliability points that will define the Pareto frontier.

Once the methodology of this dissertation is set, Chapter 4 outlines the baseline results of the architecture tool. The lunar architecture selection tool is validated against the Apollo Lunar Orbit Rendezvous, Earth Orbit Rendezvous, and Direct missions. Once the tool is validated against these historical mission modes, the ESAS final configuration will be simulated. This simulation will then be compared with the published ESAS results as well as the previous Apollo mission modes to discover any discrepancies or weakness in this design method. 
Chapter 5 contains the implementation of the lunar architecture selection tool in creating the Pareto frontiers for the different mission modes. This chapter contains the optimized Pareto frontiers for the Apollo Direct, EOR, and LOR mission modes. These optimized solutions will then be compared with the solutions obtained during the Apollo era. The ESAS architecture mission mode will also be optimized and the Pareto frontier will be compared with the actual baseline results obtained in the ESAS final report.

Chapter 6 outlines the application of this methodology to the Apollo lunar mission mode selection problem. The Pareto Frontiers created in Chapter 4 will then be compared to produce the optimal mission mode for different life cycle costs. With these Pareto Frontiers the mission mode selection made during the Apollo era can be reassessed and evaluated with this modern exploration of the design space. Once the ideal Apollo architectures are selected, the ESAS lunar architecture will be evaluated. As of 2007, the ESAS lunar mission mode is already selected as an EOR-LOR mission. The background of this selection is addressed in Chapter 1 and again in Chapter 6. Because this mission mode is already selected, the lunar architecture tool will be implemented to find the ideal architecture element configurations to produce the highest reliability architecture for any given life cycle cost.

Chapter 7 will provide a list of conclusions on the performance of the method and its implementation in the lunar architecture selection tool. Chapter 7 will also provide recommendations for future work. 


\section{CHAPTER 2}

\section{BACKGROUND}

This chapter contains the background necessary to evaluate the methodology presented in this research. This background and literature review includes a summary of current reliability practices and their strengths and shortcomings. This reliability knowledge will be used to construct an efficient dynamic fault tree analysis which is used to compile the Loss of Mission reliability and the Loss of Crew safety for each of the vehicles under consideration in a lunar architecture. This review also contains a detailed analysis of the current conceptual design process as it was conducted for the models used in the NASA Advanced Technology Lifecycle Analysis System (ATLAS) project [16]. This review will conclude with a brief overview of the multidisciplinary optimization process and a description of the Genetic Algorithm optimizer which will be used in this methodology.

\subsection{Integrated Product ANd Process Development}

The goal of any project is to design, produce, and operate a product of the highest quality and reliability at the lowest cost. As Chapter 1 discussed, the focus of this research is to provide a tool to designers that will help them optimize the reliability of each system by allocating their limited resources appropriately. The best way to achieve this is to integrate the expertise and efforts of management and engineering disciplines from the program outset [17]. This is known as Integrated Product and Process 
Development (IPPD). This process involves the use of multidisciplinary Integrated Product Teams (IPT). Members of these IPTs work on a project concurrently rather than independently. Using the IPPD approach is important since the majority of design decisions affect multiple disciplines, yet are typically made before a true understanding of their consequences is known. This is especially true in the conceptual design phase. A concept for the IPPD process created by the Georgia Institute of Technology is given below as Figure 6.

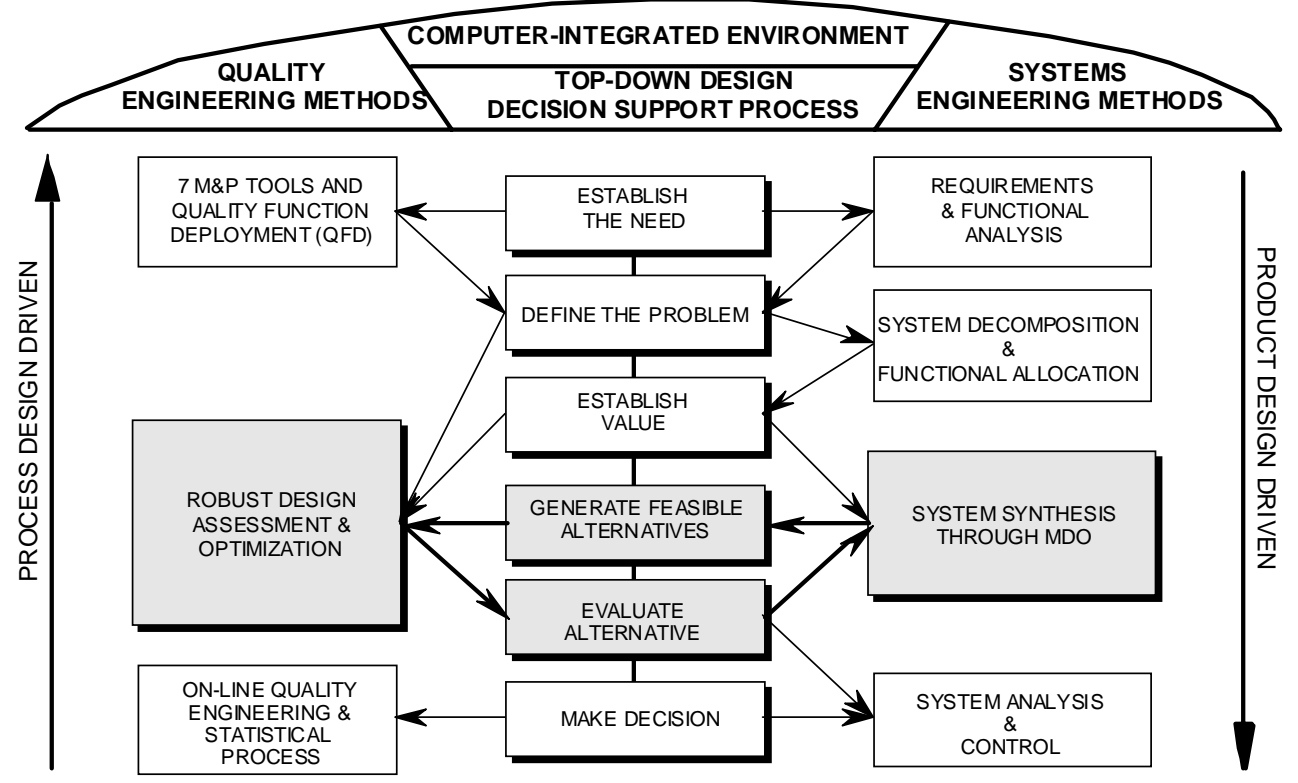

Figure 6: Georgia Tech IPPD Process [18].

As this figure indicates, the process to develop a highly reliable and successful system is complex. This process must first establish the requirements and needs of the system and then design the system to meet those needs. The majority of the research involves establishing a methodology to systematically evaluate the shaded iteration loop in Figure 
6. This process will accomplish that by evaluating the alternatives to the lunar architecture design problem and selecting the most reliable system for a given budget. The main problem with establishing the ideal solution for a lunar architecture problem is that the majority of the costs are committed early before any true details about the design are available. The proposed method will allow the user to make informed decisions on the architecture elements while most of the detailed design parameters are unknown. This will allow a paradigm shift in the typical cost, knowledge, and design freedom curves shown in Figure 7. As this figure shows, most of the costs are typically committed early in the project where little knowledge about the system is recognized yet there is pressure to select a design.

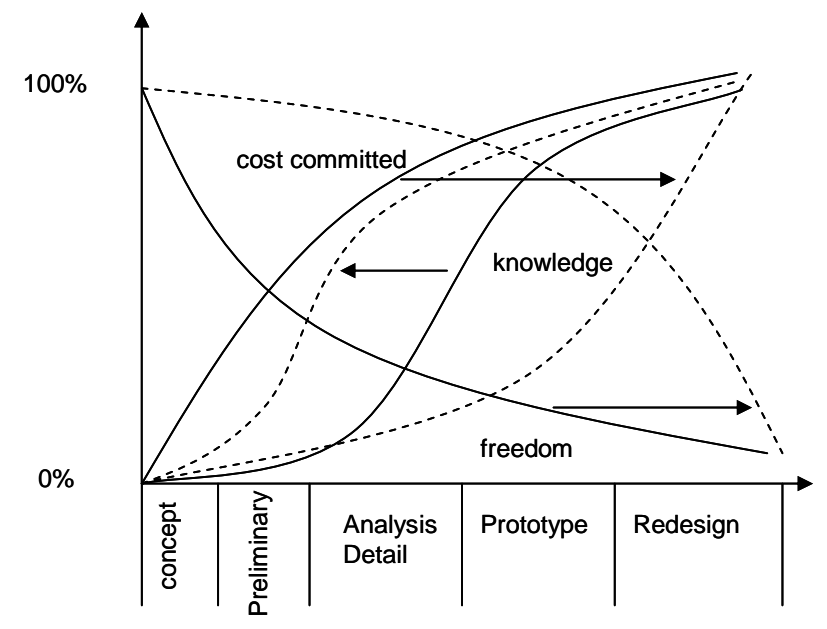

Figure 7: Paradigm Shift in Complex Vehicle Design [19].

The goal of the proposed technique is to select the appropriate architectural elements by improving the reliability knowledge in the conceptual design phase. This is accomplished by allowing the user to select the most reliable concepts that have adequate 
performance based upon the available resources. This technique, combined with the proposed uncertainty analysis, will allow the designer to have more knowledge about his design choices in the conceptual design phase. This will result in a more informed decision. The methodology will also allow the user to change his system instantaneously based upon a changing budget. This will, in turn, increase the design freedom of the user. The result of this methodology is that the user will have more knowledge and design freedom early on in the process, which will therefore reduce the number of resulting redesigns.

\subsection{RELIABILITY OVERVIEW}

As was described in the introduction, with the shrinking budget of NASA and the decreasing tolerance of failure, there has been an increased importance on improving the reliability and cost of aerospace systems. This focus on reliability is, not only to save NASA's valuable resources, but also to justify NASA's standing as one of the world's premier space agencies. Every failure experienced by NASA diminishes the prestige of the agency. Whether the failure is as mundane as a delayed launch or as catastrophic as a shuttle loss, failures are directly tied into the reliability of the system. E. Lewis defines reliability as the probability that a system will perform its intended function for a specified period of time under a given set of conditions [20]. This universal definition can be applied to anything from computer chips to spacecraft. For spacecraft, this is typically defined as the probability that a vehicle will successfully complete its mission under expected conditions. In aerospace terms this is generally broken down into two categories: loss of vehicle reliability (LOV), and loss of crew reliability (LOC). These values can be equivalent if the vehicle in question does not have a fail safe mode, such as 
a crew escape system or a programmatic plan, like that of the STS strategy, utilize the international space station in case of a tile failure. For the purposes of this research the vehicles in question will be compared based upon a LOM approach. This mainly impacts the launch vehicle selection because that tends to be the only segment where crew abort systems are possible.

There are many other terms used in the reliability research area. These include safety and security. In reliability terms, safety is defined as the ability of an entity to perform under abnormal conditions [21]. This includes failures due to outside factors, such the failure which occurred on STS 107. On that mission foam shedding from the external tank caused a failure in the Thermal Protection System (TPS). This failure in the TPS then caused a system failure and the loss of the crew. Although the foam shedding problem in itself was not a LOM event, the combination and interaction of the foam shedding along with the downstream location of the delicate TPS system caused the failure. For this example, the safety of the vehicle was diminished due to the proximity of TPS to the shedding from the external tank. For the purposes of this research, complex interactions between conceptual vehicle elements may not be completely understood. Therefore, estimates will be made about the overall vehicle reliability by accounting for the probability of complex interactions, such as those that caused the Columbia failure. Taking these facts into account, the reliability that will be used to compare the different vehicles will in fact include a qualitative estimate of safety.

A third term used in reliability analysis is security. In terms of reliability, security is defined as the ability of an entity to perform in the presence of malevolent environment [21]. This includes the ability of an aircraft to sustain battle damage and remain in 
service. For the purposes of this research, security will not be considered in the selection process.

Risk is another term commonly used in aerospace design. Risk is defined as the potential for loss resulting from exposure to a danger. Risk is considered a measure of both safety and security. NASA defines risk in a set of triplets. Determining risk generally amounts to answering the following questions [22]:

\section{What can go wrong?}

2. How likely is it?

3. What are the consequences?

The answers to these questions help designers understand the risks associated with their designs. The answer to the first question is a set of accident scenarios. The second and third questions evaluate the probabilities of the scenarios as well as their impact on the failure. An implementation of this risk definition is shown in Figure 8.

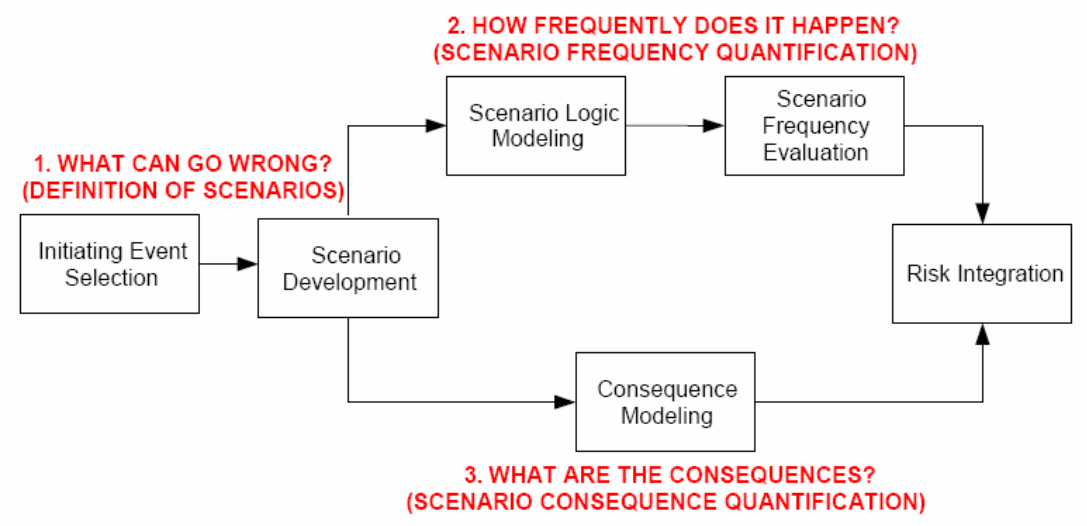

Figure 8: Implementation of the Triplet Definition of Risk used by NASA [23]. 
This figure shows the development of a Probabilistic Risk Assessment (PRA), as defined by NASA. To properly gauge all of the risks associated with a design, the designer must first understand all of the failure modes associated with the vehicle. This is truly a daunting task since complex systems such as space vehicles have millions of components, all of which need to interact properly to produce a working system.

For this proposed research, only failures that can cause a loss of vehicle will be analyzed. This is done so that the likelihood of catastrophic event can be characterized and used to compare competing vehicles for lunar exploration architecture. Reliability analysis is an important tool in this risk assessment process. Reliability analyses provide the answers to the first two questions in the risk triplet. The subsequent reliability analyses described in this section can be used to identify problem areas in a design and determine how likely each designated failure scenario is. From this analysis a comprehensive risk assessment can be formulated to compare competing designs.

\subsection{RELIABILITY ANALYSIS SHORTCOMINGS}

Reliability and safety are generally considered some of the most important design parameters in the aerospace field. The importance has only increased recently with the loss of a second Space Shuttle crew in the Columbia disaster. Unfortunately, reliability is one of the hardest design parameters to fully characterize in the conceptual design process. This is mainly because a majority of the interactions between the subsystems of the vehicle are not completely understood. These interactions play a major role in the overall reliability of the system. 


\subsubsection{AIRCRAFT RELIABILITY}

Despite the problems with addressing reliability early in the design process, attempts have been made to increase the reliability of aerospace systems since the early 1900s. After World War I, reliability became of major interest to the aircraft industry and attempts to improve reliability were pushed to the forefront. These attempts usually involved trial and error techniques with little emphasis given to quantitative reliability techniques [24]. As the aircraft design process was refined throughout the 1940s and 50s an emphasis was placed on collecting data on system failures to better quantify the probability of such failures occurring. This data collection process was extensive but easily obtained with the variety of aircraft operating at the time. This data collection allowed the aircraft designers of the day to properly categorize the reliability problems experienced by their aircraft. This wealth of data enabled the designers to identify the weak components of the system and develop newer more reliable components to act as replacements. In cases where the weak components could not be improved upon, redundancy was added to increase the reliability of the entire system. These techniques of categorizing failures and refining designs continued for decades, with each refinement creating a more reliable system. 


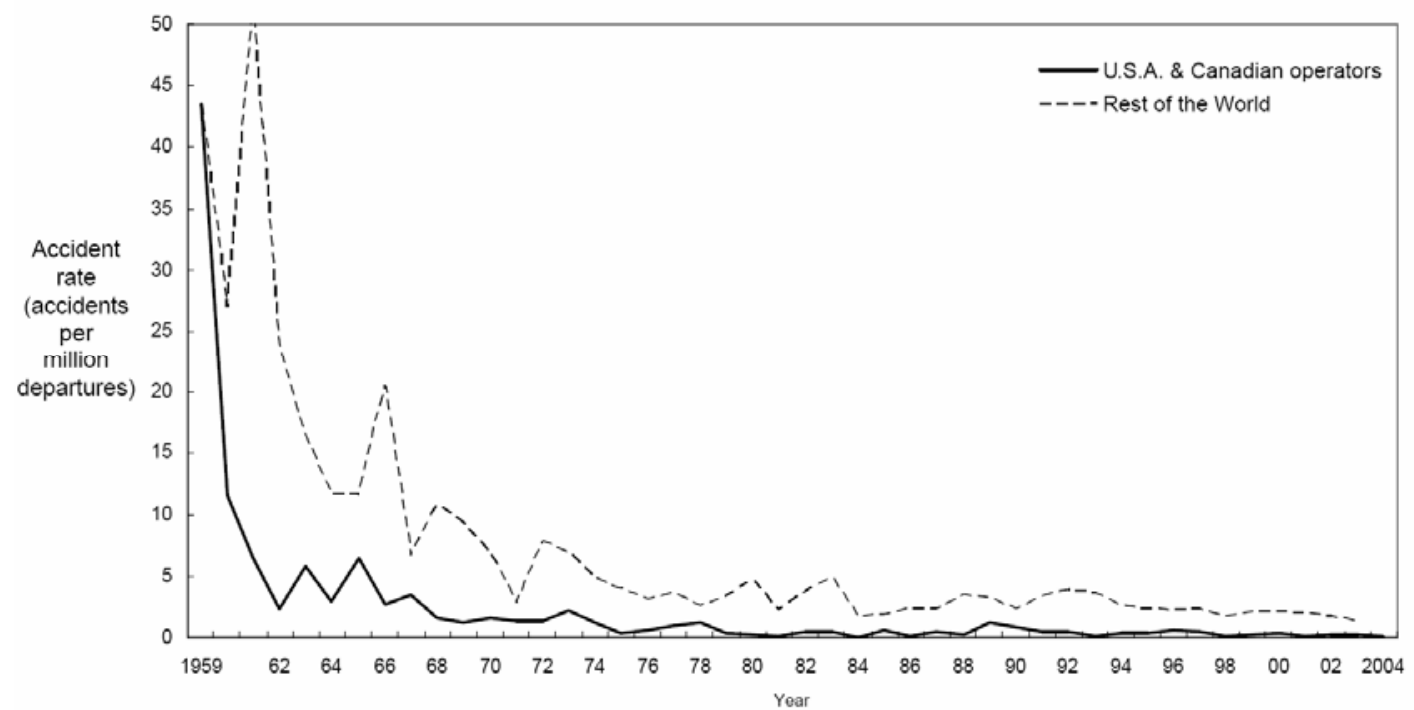

Figure 9: Catastrophic Accident Rate (Hull Loss) of Worldwide Commercial Jet Fleet (1959-2004) [25].

This figure demonstrates how the increasing reliability gained through this data collection process reduced the number of catastrophic failures in the airline industry. This reduction of accidents is the direct result of the refinement instituted by the airline designers as they began to understand the failure modes of their vehicles. To achieve this increased reliability (accident rate of 1E-7), hundreds of millions of flights had to be conducted and the resulting data analyzed and applied to the new aircraft designs.

The space industry of today is similar to the early aircraft industry in that there are a limited number of vehicles operating. In fact there are only three active man rated vehicles in the world today: the Space Shuttle, the Soyuz vehicle, and the Shenzhou vehicle. This is a major problem with applying the aircraft reliability model to space systems. Although similar, the space transportation problem is fundamentally different than that faced by the airplane community. The environment faced by space vehicles is 
orders of magnitude more difficult to manage than it's the aircraft counterparts. This results in a completely new problem for spacecraft designers, and the reliability data is therefore limited.

\subsubsection{SPACE SHUTtLE RELIABILITY}

Risk assessment and reliability analysis have been a part of NASA's procedures since the agency was founded. Unfortunately, during the Apollo program, pessimistic risk estimates discouraged the agency from adopting a quantitative risk analysis [26]. In fact, the overall mission success probability was only $40.43 \%$, with a mission safety of only 85.00\% [27]. After Apollo when this number seemed overly pessimistic, NASA abandoned quantitative risk analysis for a more qualitative approach. As a result, the Space Shuttle design process described in Chapter 1 was reliant on cost and performance as the main design drivers. The inherent dangers associated with space travel as well as the increased importance placed upon mitigating these risks, has forced NASA to place increased importance on reliability.

This increased importance is apparent in the shuttle risk assessments conducted since the first shuttle accident, Challenger in 1986, and continuing through the Columbia accident in 2003. After the Challenger accident, it became obvious that the Space Shuttle reliability programs were lacking and a new focus on reliability was needed. The aircraft model of failure analysis was not appropriate due the limited number of flights and the different environment of space travel. To accomplish an accurate reliability analysis, shuttle program managers needed to address the failure modes of the Space Shuttle. This

involved a top down breakdown of each event that could cause a shuttle failure and subsequently applying a failure analysis to each event [28]. These failure analyses 
required detailed data regarding the possible failures of the hundreds of components that comprise the Space Shuttle system. Due to the limited flight experience of the Space Shuttle, test data had to be included in the reliability estimates [29]. This resulting analysis was completed under a 1995 study conducted by Science Applications International Corporation (SAIC). This study was comprehensive quantitative risk assessment that included all phases of a shuttle mission [30]. This risk assessment concluded that the LOV probability of the shuttle lie between $1 / 76$ and $1 / 230$. This seemed accurate based upon the limited number of flights conducted.

The Columbia accident of 2003 resulted in a renewed emphasis on reliability. The Columbia Accident Investigation Board made two significant findings regarding the proposed research and shuttle risk assessment. First, it found that over the last two decades, little to no progress has been made toward attaining integrated, independent, and detailed analyses of risk to the Space Shuttle. Second, it found that system safety engineering and management is separated from mainstream engineering and is not vigorous enough to have an impact on system design [14]. These organizational problems are the result of NASA's previous design methodologies, which focused on performance and cost. The new emphasis on reliability and safety will force a new design process and current aerospace designers will be required to include the analysis of reliability and safety in their design proposals.

The shuttle program has been forced to increase the reliability of an unnecessarily complex system through patchwork modifications of the existing vehicle. Most of the reliability estimates of the Space Shuttle program were made after the system was operational. The lunar exploration problem proposed by President Bush in his Vision for 
Space Exploration is a much more complicated mission and therefore will result in a more complex architecture. The proposed research attempts to address the problems faced by the reliability of the shuttle program by fully integrating the reliability estimates into the design process and including them as the major discriminators in the conceptual design. This will allow the resulting vehicles that comprise the lunar architecture to be designed specifically to attain the maximum reliability for the given budget.

\subsection{RELIABILITY TECHNIQUES}

This section will describe the relevant techniques of reliability and risk assessment currently applied in aerospace design. Many techniques have been implemented to estimate the reliability of complex systems. The earliest techniques involved observation of operational vehicles. These gave accurate representations of the overall failure rates, but did little to increase knowledge about the interactions of complex subsystems to better understand the reliabilities of systems. Quantitative reliability studies became a reality in World War II. A German mathematician, named Lusser, questioned the existing notion that the reliability of a chain of components was determined by the strength of its weakest link. He showed that a series of strong links can be inherently more unreliable than a single weak link, due to the operational loading. His theories resulted in an increase in quality assurance of all the vehicle components and increased the reliability of the V1 missile to a success rate of over 60 percent [24]. This was the beginning of current reliability theories and their applications to complex systems. 


\subsubsection{FAILURE MODE EFFECTS AND CRITICALITY ANALYSIS}

Failure Mode Effects and Criticality Analysis (FMECA) is a qualitative reliability analysis that employs a set procedure for the evaluation of the severity of potential failure modes. FMECA is an extension of Failure Mode Effects Analysis (FMEA), which then ranks each potential failure mode according to severity and probability of occurrence. The objective of this analysis is to identify weak areas in the design by breaking the systems into subsystems and then to components to identify all of the possible failure modes.

The purpose of FMECA is to identify the potential failures in the system so that they can be addressed. According to MIL-STD-1629A, the procedure for evaluation FMEA of a complex system is to decompose the problem to the component level with each component or subassembly treated as a black box. The reliability engineer then evaluates the effect of the loss of a black box on the rest of the system. The FMECA process is an organized brainstorming activity to rank the severity of the failure modes of the different subassemblies on the entire system.

In the aerospace design process, the FMECA process begins with a Functional Hazard Analysis (FHA). FHA is a systematic process that defines the system and the potential hazards in the total system. FHA is designed to develop safety design to guidelines in order to reduce or even eliminate potential hazards from the conceptual design. The hazards identified in the FHA process are then qualitatively prioritized by probability of occurrence and by the risk assessment code shown in Table 2 . 
Table 2: Hazard Severity Classification for FHA [17].

\begin{tabular}{|c|c|c|c|}
\hline Hazard Severity & Category & Hazard Severity Definition & $\begin{array}{c}\text { Design } \\
\text { Standard }\end{array}$ \\
\hline Catastrophic & 1 & $\begin{array}{l}\text { Loss of equipment or personnel } \\
\text { death, or severe environmental } \\
\text { damage }\end{array}$ & $\begin{array}{l}\text { Extremely } \\
\text { Improbable }\end{array}$ \\
\hline $\begin{array}{l}\text { Hazardous or } \\
\text { Critical }\end{array}$ & 2 & $\begin{array}{l}\text { Major Equipment damage or severe } \\
\text { injury to personnel, or major } \\
\text { environmental damage }\end{array}$ & $\begin{array}{c}\text { Extremely } \\
\text { Remote }\end{array}$ \\
\hline $\begin{array}{l}\text { Major or } \\
\text { Marginal }\end{array}$ & 3 & $\begin{array}{l}\text { Minor equipment damage or minor } \\
\text { injury to personnel, or minor } \\
\text { environmental damage }\end{array}$ & Improbable \\
\hline $\begin{array}{l}\text { Minor or } \\
\text { Negligible }\end{array}$ & 4 & $\begin{array}{l}\text { Insignificant equipment damage or } \\
\text { injury to personnel, or environmental } \\
\text { damage }\end{array}$ & Probable \\
\hline
\end{tabular}

This severity classification is combined with the probability of occurrence to form a Risk Assessment Code (RAC). RAC is a useful graphical tool to determine whether a risk is acceptable or whether major redesign work must be done to raise the design's safety to an acceptable level. An example of both the severity classifications and the RAC are given as Table 3 and Table 4.

Table 3: Risk Assessment Code Matrix [17].

\begin{tabular}{|c|c|c|c|c|c|}
\hline \multirow[t]{2}{*}{ Hazard Severity } & \multicolumn{5}{|c|}{ Probability of Occurrence } \\
\hline & A & B & C & D & $\mathrm{E}$ \\
\hline 1 & 1 & 1 & 2 & 3 & 4 \\
\hline 2 & 1 & 2 & 3 & 3 & 5 \\
\hline 3 & 2 & 3 & 4 & 5 & 5 \\
\hline 4 & 3 & 4 & 5 & 5 & 5 \\
\hline
\end{tabular}


Table 4: Hazard Probability of Occurrence for FHA [17].

\begin{tabular}{|c|c|c|c|}
\hline $\begin{array}{c}\text { Hazard } \\
\text { Occurrence }\end{array}$ & Level & $\begin{array}{c}\text { Probability of Hazard Occurrence } \\
\text { Definition }\end{array}$ & $\begin{array}{l}\text { Expected } \\
\text { Experience }\end{array}$ \\
\hline Frequent & A & $\begin{array}{l}\text { Likely to occur frequently in operating } \\
\text { life of the system }\end{array}$ & $\begin{array}{l}\text { Continuously } \\
\text { experienced }\end{array}$ \\
\hline Probable & B & $\begin{array}{l}\text { Will occur several times in operating } \\
\text { life of the system }\end{array}$ & $\begin{array}{l}\text { Will occur } \\
\text { frequently }\end{array}$ \\
\hline Occasional & $\mathrm{C}$ & $\begin{array}{l}\text { Likely to occur sometime in operating } \\
\text { life of the system }\end{array}$ & $\begin{array}{l}\text { Will occur } \\
\text { several times }\end{array}$ \\
\hline Remote & D & $\begin{array}{c}\text { Possible to occur in operating life of the } \\
\text { system }\end{array}$ & $\begin{array}{l}\text { Reasonably } \\
\text { expected to } \\
\text { occur }\end{array}$ \\
\hline Improbable & E & $\begin{array}{l}\text { Assumed occurrence may not be } \\
\text { experienced in the operating life of the } \\
\text { system }\end{array}$ & $\begin{array}{l}\text { Unlikely to } \\
\text { occur }\end{array}$ \\
\hline
\end{tabular}

The RAC matrix gives a code for the resulting risk of the activity. Red boxes denote an undesirable hazard that must be corrected through redesign. A yellow box denotes a hazard that must be addressed by the operator of the vehicle. This risk may either be deemed acceptable or a request for redesign must be made. A green box denotes a risk that is generally considered acceptable within the aerospace community.

Once the FHA has been completed, the FMECA procedure evaluates the results of the FHA and assesses the impact on the total system. This can be accomplished through a series of functional block diagrams of the system and subsystem components. Functional blocks are outlined and the interactions are shown. Once the block diagrams are created and the interactions known, a FMECA worksheet can be created. 


\begin{tabular}{|c|c|c|c|c|c|}
\hline PROGRAM: NRA8-30 TA-1 NAS8-01097 & \multicolumn{3}{|c|}{$\begin{array}{c}\text { FHA/FMECA } \\
\text { Orbital Sciences Corp oration (OSC) }\end{array}$} & \multicolumn{2}{|l|}{ Date: } \\
\hline \multirow{2}{*}{ 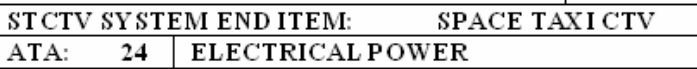 } & \multirow{2}{*}{ SPACE TAXI CTV } & LOV & \multirow{2}{*}{$\begin{array}{cl}\text { LOV: } & \begin{array}{l}1085 \times 10^{6} \\
\text { LOV: }\end{array} \\
105 \times 10^{-6}\end{array}$} & \multicolumn{2}{|c|}{ Orig: 25 Oct 01} \\
\hline & & $\mathbf{L}$ & & \multicolumn{2}{|c|}{ Rev 4: 6 Mar 02} \\
\hline \multicolumn{6}{|l|}{ SYSTEM DEFINITION } \\
\hline \multirow{2}{*}{$\begin{array}{c}\text { l } \\
\text { SY STEM HAZARD AND } \\
\text { FAILURE MODE IDENTIFICATION }\end{array}$} & 2 & 3 & \multicolumn{2}{|l|}{4} & 5 \\
\hline & $\begin{array}{c}\text { OPS. } \\
\text { PHASE }\end{array}$ & $\begin{array}{c}\text { EFFECT ON } \\
\text { OTHER SY STEMS }\end{array}$ & $\begin{array}{l}\text { EFFECT OF HA } \\
\text { FAILURE M } \\
\text { VEHIC }\end{array}$ & $\begin{array}{l}\text { AND } \\
\text { ON }\end{array}$ & $\begin{array}{l}\text { CLASS \& } \\
\text { CF PROB }\end{array}$ \\
\hline $\begin{array}{l}\text { 1. Inadequate electrical power to meet EMA and systems } \\
\text { requirements }\end{array}$ & 2 thru 14 & No power to systems & \multicolumn{2}{|c|}{$\begin{array}{l}\text { Potential mission abort or loss } \\
\text { of vehicle }\end{array}$} & $\begin{array}{c}I \\
48 \times 10^{-6}\end{array}$ \\
\hline $\begin{array}{l}\text { 2. Electrical system failure due to inadequate or improper } \\
\text { electrical power grounding, bonding, shielding and wiring } \\
\text { separation }\end{array}$ & 1 thru 16 & No power to systems & \multicolumn{2}{|c|}{$\begin{array}{l}\text { Potential mission abort or loss } \\
\text { of vehicle }\end{array}$} & $\begin{array}{c}\mathrm{I} \\
28 \times 10^{-6}\end{array}$ \\
\hline $\begin{array}{l}\text { 3. Electrical system failure due to br oken wire, connectors, } \\
\text { terminal blocks, wiring insulation chaffing, wire gauge or } \\
\text { me chanical stress on wire or connectors corrosion }\end{array}$ & 1 thru 16 & No power to systems & \multicolumn{2}{|c|}{$\begin{array}{l}\text { Potential mission abort or loss } \\
\text { of vehicle }\end{array}$} & $\underset{18 \times 10^{-6}}{\mathrm{I}}$ \\
\hline $\begin{array}{l}\text { 4. No power to } 28 \mathrm{VDC} \text { bus due to battery or distribution } \\
\text { system failure, or ex cessive leak rate, or improper Orbiter } \\
\text { power interface }\end{array}$ & 2 thru 14 & No power to systems & \multicolumn{2}{|c|}{$\begin{array}{l}\text { Potential mission abort or loss } \\
\text { of vehicle }\end{array}$} & $\underset{6 \times 10^{6}}{I}$ \\
\hline $\begin{array}{l}\text { 5. No power to } 270 \text { VDC bus due to battery or distribution } \\
\text { system failure, or ex cessive leak rate, or improper Orbiter } \\
\text { power interface }\end{array}$ & 10 thru 14 & No power to systems & \multicolumn{2}{|c|}{$\begin{array}{l}\text { Potential mission abort or loss } \\
\text { of vehicle }\end{array}$} & $\begin{array}{c}\mathrm{I} \\
5 \times 10^{6}\end{array}$ \\
\hline $\begin{array}{l}\text { 6. System failure due improper ground batteries servicing } \\
\text { or improper interface withISS electrical power }\end{array}$ & 1 thru 16 & $\begin{array}{l}\text { Intermittent com mand to } \\
\text { systems or limited system } \\
\text { perform ance }\end{array}$ & \multicolumn{2}{|c|}{$\begin{array}{l}\text { Degraded vehicle performance } \\
\text { leading to mission ground or } \\
\text { flight abort }\end{array}$} & $\begin{array}{c}\text { II } \\
0 \times 10^{-6}\end{array}$ \\
\hline $\begin{array}{l}\text { 7. Battery failure due to internal short, overheating or } \\
\text { explosion of battery, or inadequate durability }\end{array}$ & 1 thru 16 & $\begin{array}{l}\text { Inadequate or limited } \\
\text { system performance }\end{array}$ & \multicolumn{2}{|c|}{$\begin{array}{l}\text { Degraded vehicle performance } \\
\text { leading to mission ground or } \\
\text { flight abort }\end{array}$} & $\begin{array}{c}\text { II } \\
0 \times 10^{-6}\end{array}$ \\
\hline $\begin{array}{l}8 \text { Electrical system, battery, LRUs, parts and wiring failure } \\
\text { due to lightning strike or weather }\end{array}$ & 1 thru 16 & $\begin{array}{l}\text { Inadequate or limited } \\
\text { system performance }\end{array}$ & \multicolumn{2}{|c|}{$\begin{array}{l}\text { Degrade d vehicle performance } \\
\text { leading to mission ground or } \\
\text { flight abort }\end{array}$} & $\begin{array}{c}\text { II } \\
0 \times 10^{-6}\end{array}$ \\
\hline $\begin{array}{l}\text { 9. Noisy electrical power buses che to internal or external } \\
\text { EMI RFI, and space radiation }\end{array}$ & 1 thru 16 & $\begin{array}{l}\text { Intermittent com mand to } \\
\text { systems or limited system } \\
\text { perform ance }\end{array}$ & $\begin{array}{l}\text { Degrade d vehicle } \\
\text { leading to mission } \\
\text { flight abort }\end{array}$ & $\begin{array}{l}\text { ormance } \\
\text { und or }\end{array}$ & $\begin{array}{c}\text { II } \\
0 \times 10^{-6}\end{array}$ \\
\hline $\begin{array}{l}\text { 10. Battery, LRUs and parts failure due to vibration, } \\
\text { shock, acoustics, temperature, pressure, humidity or } \\
\text { inadequate reusability }\end{array}$ & 1 thru 16 & $\begin{array}{l}\text { Intermittent com mand to } \\
\text { systems or limited system } \\
\text { perform ance }\end{array}$ & $\begin{array}{l}\text { Degrade d vehicle } \\
\text { leading to mission } \\
\text { flight abort }\end{array}$ & int or & $\begin{array}{c}\text { II } \\
0 \times 10^{-6}\end{array}$ \\
\hline $\begin{array}{l}\text { 11. Electrical system failure due to inchuced maintenance } \\
\text { by maintenance crew, of GSE or servicing equipm ent, no } \\
\text { or inade quate procedures or per sonnel training }\end{array}$ & $\begin{array}{c}1,14,15 \\
16\end{array}$ & $\begin{array}{l}\text { Potential loss of systems } \\
\text { function Potential injury } \\
\text { to support personnel }\end{array}$ & $\begin{array}{l}\text { Operations stopps } \\
\text { mission delay or }\end{array}$ & $\begin{array}{l}\text { ading to } \\
\text { ad abort }\end{array}$ & $\begin{array}{c}\text { II } \\
0 \times 10^{-6}\end{array}$ \\
\hline 12. Electrical shock to maintainer & $\begin{array}{c}1,14,15 \\
16\end{array}$ & $\begin{array}{l}\text { Potential irjury to support } \\
\text { personnel }\end{array}$ & $\begin{array}{l}\text { Operations stoppe } \\
\text { mission delay or }\end{array}$ & $\begin{array}{l}\text { ading to } \\
\text { ad abort }\end{array}$ & $\begin{array}{c}\text { II } \\
0 \times 10^{-6}\end{array}$ \\
\hline $\begin{array}{l}\text { OPERATION/MISSI ON PROFII } \\
1 \text { = Pre-flight; } 2 \text { = Launch pad } / \text { take-off; } 3=\text { Ascent; } 4 \text { = } \\
\text { RL V-O/STCTV to orbit; } 6=\text { RL V-O/STC TV separat } \\
\text { dep byment; } 9 \text { = STCTV dockun-dock; } 10=\text { RL V-O or } \\
\text { Descent; } 12 \text { = Land ing; } 13=\text { Rolbut; } 14 \text { = Post-land ing } \\
\text { System integration }\end{array}$ & $\begin{array}{l}\text { E SEGMEI } \\
\text { MRRV RL } \\
\text { n; } 7 \text { = ST } \\
\text { TV, or Log } \\
15=\text { Grou }\end{array}$ & $\begin{array}{l}\text { S: } \\
\text { BRL V-O sep aration; } 5= \\
\text { V in orbit; } 8=\text { Payb ad } \\
\text { ics Vehicles de-orbit; } 11= \\
\text { operations } 16 \text { = STCTV }\end{array}$ & $\begin{array}{r}\text { SE VERI } \\
\text { CLASSIFIC } \\
\text { I = CATASTROP } \\
\text { II = HAZARD OU } \\
\text { III = MAJOR } \\
\text { IV = MINOR } \\
\end{array}$ & & $\begin{array}{l}\text { REFERENCE } \\
\text { a) MSFC-R } \\
\text { b) STCTV T } \\
\text { c) } 931-S E 01 \\
\text { d) } 931-S E 01 \\
\text { e) } 931-S E-0 \\
\end{array}$ \\
\hline
\end{tabular}

Figure 10: FMECA Worksheet for CTV [17].

Figure 10 shows an example FMECA worksheet for a Crew Transfer Vehicle (CTV). As this worksheet demonstrates, the analysis can be very detailed even in the conceptual design phase. All of the potential hazards are outlined for the power system of the CTV with severity indicated by a color code. Overall, the FMECA process is used to identify all of the possible failure modes of the systems. This allows the designer to address the 
most critical failure modes and subsequently redesign the vehicle or choose a different vehicle for the mission.

\subsubsection{RELIABILITY NETWORKS AND REDUNDANCY MODELING}

Another technique commonly used in reliability is the implementation of networks and redundancy modeling. A reliability network is defined as a representation of the reliability dependencies between components in a system [24]. These networks give a graphic representation of the system and are one of the simplest ways to quantitatively calculate the reliability of a system. These simple networks use basic

probability analyses to compute the overall reliability of a system. These reliability networks, also known as Reliability Block Diagrams (RBD), provide a simple method to model a system of individual components. A simple reliability network is given below as Figure 11.

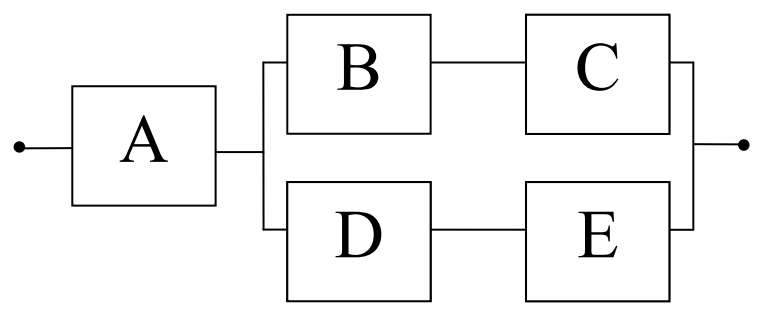

Figure 11: Simple Reliability Network.

As this figure shows, component A must be functional for the system to work successfully. If $\mathrm{A}$ is working, then either components $\mathrm{B}$ and $\mathrm{C}$ or components $\mathrm{D}$ and $\mathrm{E}$ have to be operational for the system to be functional. 
Reliability Block Diagrams are a simple way to model components working together to create a system. As systems get more complex, the reliability block diagram techniques tend to break down and become very complicated. RBDs are usually used to model subsystems to simplify both the models and the calculations. These models rely on an accurate description of the component reliabilities as well as the independence of the components modeled. RBDs can be used to model almost any system as long as it can be broken down to its independent components. The RBDs can help to identify weak points in the system and allow the designer to improve the system by either increasing the reliability of the component or adding redundancy to the system.

Redundancy is a method of increasing reliability of the system by including a backup, or identical component, to the system that will take over if the original component fails. Redundancy is not a solution to all reliability problems since redundant systems usually increase weight, cost, and complexity. Redundancy is a good solution for backing up inherently unreliable components that are essential to the success of the system. There are four approaches to adding redundancy to a design [17]:

1. Active Redundancy- Redundant systems operate simultaneously.

2. Voting Redundancy- Multiple components function and the one that produces the expected results is chosen to work with the system.

3. Stand-by Redundancy- The backup component stays offline until a failure occurs. The backup then begins to operate in place of the original component when a failure occurs. 
4. Redundancy with Repair- In this configuration a failed component can be repaired before the mission is lost, or the failed component is not critical to the mission success.

These redundancy models can be combined in many ways to produce a more reliable system. Other redundancy configurations include parallel systems, duplex, bimodal, majority voting, adaptive majority logic, gate connector, and stand-by. These are all described in detail in reference 17.

The Space Shuttle can be depicted as an example of a breakdown of a complex system into its subsystems. During the Challenger accident in 1986, an O-ring failure in the solid rocket motor caused a system wide failure ending in a loss of crew. The designers of the Space Shuttle realized the potential problem with O-ring joints and added stand-by redundancy to the system by adding a second O-ring to each joint of the system. The resulting block diagram of the 12 O-rings working in redundant pairs on the six joints (three per solid rocket booster) is shown below.

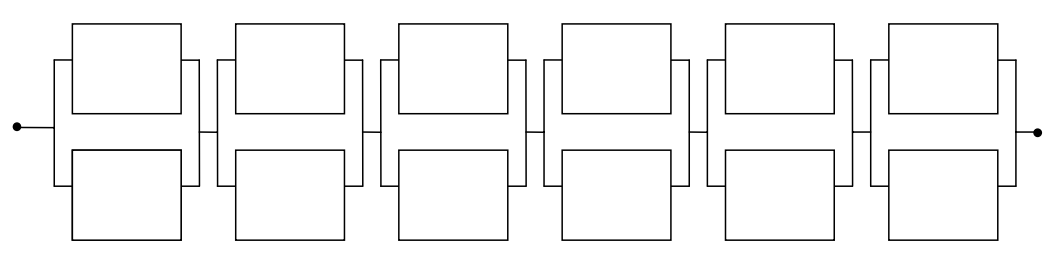

Figure 12: Proposed O-Ring RBD for Space Shuttle [31].

This redundancy was based on the O-ring failure and was designed to be completely independent. Unfortunately, as the solid rocket motors fire, the steel structure bulges due to the increased pressure and temperature. This bulge causes a rotation in the four joints 
of the solid rocket motor. The rotation then causes a gap to form where the O-rings were intended to seal. This gap affected both O-rings and caused interdependence between the O-rings. This interdependence causes the overall reliability of the joint to be far less than the simple model in Figure 12 predicts. The misrepresentation by the model has been fixed and the resulting differences in reliability were calculated. The results of analyses of the failures of the O-rings due to temperature were drastic. The report found that the Space Shuttle had a launch reliability of 0.98 when the temperature was above 60 degrees Fahrenheit, but only 0.87 when the temperature was below 31 degrees Fahrenheit [31]. This discrepancy was a direct result of the O-ring reliability.

As this example demonstrates, RBD can be useful but does not always address all of the interdependencies that can exist in a complex system. That is why RBD and redundancy methods are typically used early in the design process to understand the top level reliability relationships. More detailed analysis is needed to adequately represent the true reliability of a complex system prior to the flight testing.

\subsubsection{FAULT TREE ANALYSIS}

Fault trees are another common reliability technique used by conceptual designers. A Fault Tree Analysis (FTA) is a top-down or deductive approach to reliability modeling. This approach involves identifying the top level failure and uses an approach to identify potential causes for a system failure.

FTA was first developed in 1962 by Bell Telephone Laboratories for use in the Minuteman ballistic missile system. In this case a top level event, such as a missile failure, is broken down into the causes that can lead to the failure. These causes are 
further broken down into the failures of systems and then subsystems until the component level probability of failure is established. These component failures can then be combined mathematically to compute the overall reliability of the system. A simple FTA consists of two types of gates: an 'AND' gate and an 'OR' gate. In this simplest form of FTA, an 'AND' gate denotes that the output event (higher level) occurs if all events inputting to it (below) occur. An 'OR' gate denotes that the output event will occur if any of the input events occur. Other FTAs can include more complicated gates such as a 'k out of n' gate (voting gate), 'inhibit' gate, 'not' gate, 'Exclusive OR' gates, and 'Priority AND' gates [24].

A FTA for a typical conceptual design involves multiple layers but does not go into the detail of a FTA for a preliminary design. An example of a FTA for a conceptual lunar lander, named Artemis, is given below.

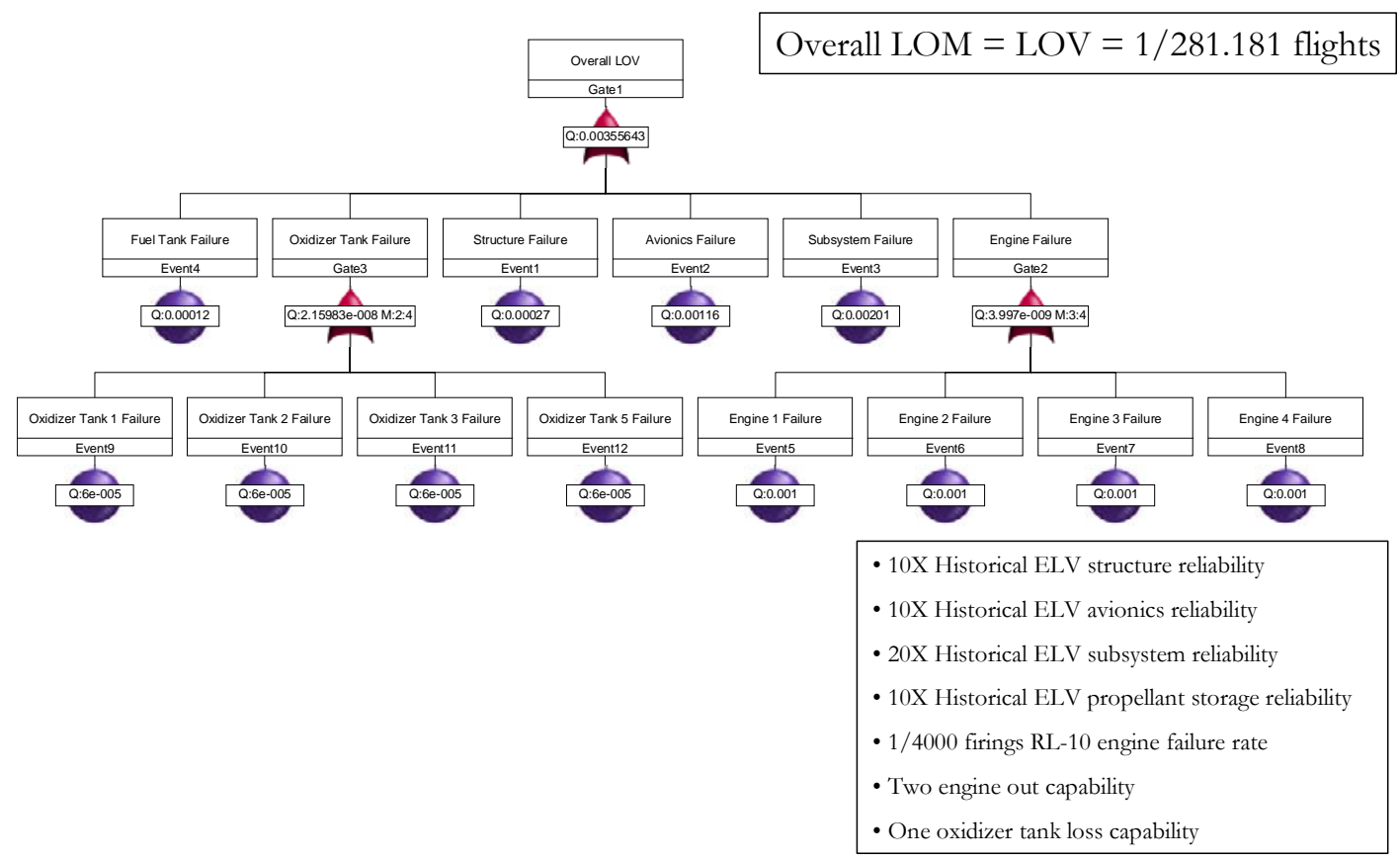

Figure 13: Fault Tree Analysis for Artemis Conceptual Lunar Lander Design [32]. 
Artemis was a reusable lunar lander designed by the Space Systems Design Laboratory (SSDL) at Georgia Tech. The FTA shown in Figure 13 is a typical example of the FTA conducted at the conceptual vehicle design level. This analysis was conducted in a FTA software tool called Relex [33]. This FTA used the assumptions in the box to compute the component reliabilities in the blue circles. These component reliabilities can typically be computed by similar subsystem FTAs. The component reliabilities for this vehicle were then combined in the FTA to produce an overall vehicle reliability of 0.996 for the mission of landing on the moon and returning to Low Lunar Orbit (LLO).

Fault tree analyses can also become rather complicated when applied to a complex system. For example, the Space Shuttle fault tree has over 1,400 elements. This FTA is broken down into 7 branches each containing the different components that make up the failure probabilities. A summary of the fault tree presented in the Columbia investigation is provided as Table 5 .

Table 5: Elements in the Space Shuttle Fault Tree [14].

\begin{tabular}{lcccc}
\hline Branch of Fault Tree & $\begin{array}{c}\text { Total } \\
\text { Number of } \\
\text { Elements }\end{array}$ & $\begin{array}{c}\text { Number of Open Elements } \\
\text { Likely }\end{array}$ & Possible & Unlikely \\
Orbiter & 234 & 3 & 8 & 6 \\
Space Shuttle Main Engine & 22 & 0 & 0 & 0 \\
Reusable Solid Rocket Motor & 35 & 0 & 0 & 0 \\
Solid Rocket Booster & 88 & 0 & 4 & 4 \\
External Tank & 883 & 6 & 0 & 135 \\
Payload & 3 & 0 & 0 & 0 \\
Integration & 295 & 1 & 0 & 1 \\
\hline
\end{tabular}


As this table shows, the FTA for the Space Shuttle was incredibly complex yet still missed 10 likely elements that could cause a failure. This table demonstrates one of the main problems of an FTA. If components are ignored or forgotten in the bottom levels, the reliability calculated at the top level is artificially inflated. This can cause a system to be deemed acceptably reliable even though realistically the reliability is optimistically high. FTA also suffers from the same requirement of independency of root events that affects the RBD analysis.

\subsubsection{MARKOV ANALYSIS}

A Markov analysis is another tool used by engineers to evaluate the reliability of a system. Unlike FTA and RBD, this analysis does not require that the component events be completely independent. This makes Markov analysis a truly dynamic state space model.

A Markov analysis consists of a series of Markov chains. These chains consist of states and transitions from the states. A state is the condition in which the element is currently operating. This is typically described in reliability analysis as operating or failed. The transitions between the states are usually described as the failures and repairs of the system. A simple Markov chain for a parallel system is given below.

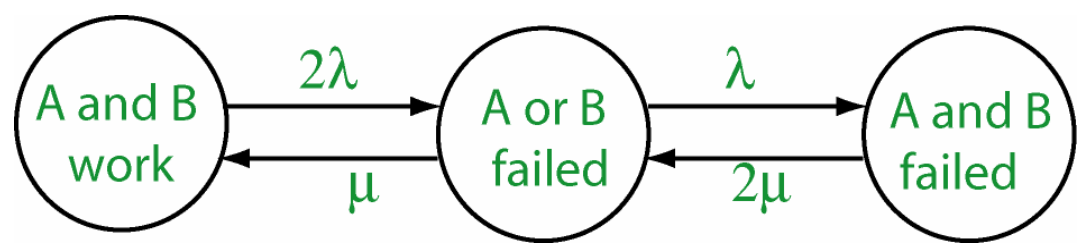

Figure 14: Markov Chain of a Parallel System with Two Identical Components [21]. 
In this type of model the circles indicate the states and the transitions are denoted by arrows. This example also shows that the transitions occur with a failure rate of $\lambda$ and a repair rate of $\mu$. These symbols are used because the typical Markov chains model the failure rates as exponential functions. This is because the Markov chain requires that the system have the memoryless property. The memoryless property states that the future states of the problem are independent of the past states. For example, a repaired component has the same likelihood of failure as a brand new component. This is described mathematically as equation 1 .

$$
P\left(X_{t+d t}=k \mid X_{t}=j, X_{t-d t}=i, X_{t-2 d t}=h, \ldots, X_{0}=a\right)=P\left(X_{t+d t}=k \mid X_{t}=j\right)
$$

The above equation shows that the probability of making a transition must be constant with time, or the likelihood of failure is only dependant on the working state of the component.

One of the main drawbacks of the Markov analysis is that the size of the model increases exponentially with the number of components in the model. For example a Markov model for a simple 2 out of 3 system with warm spares and repairs is given as Figure 15. 


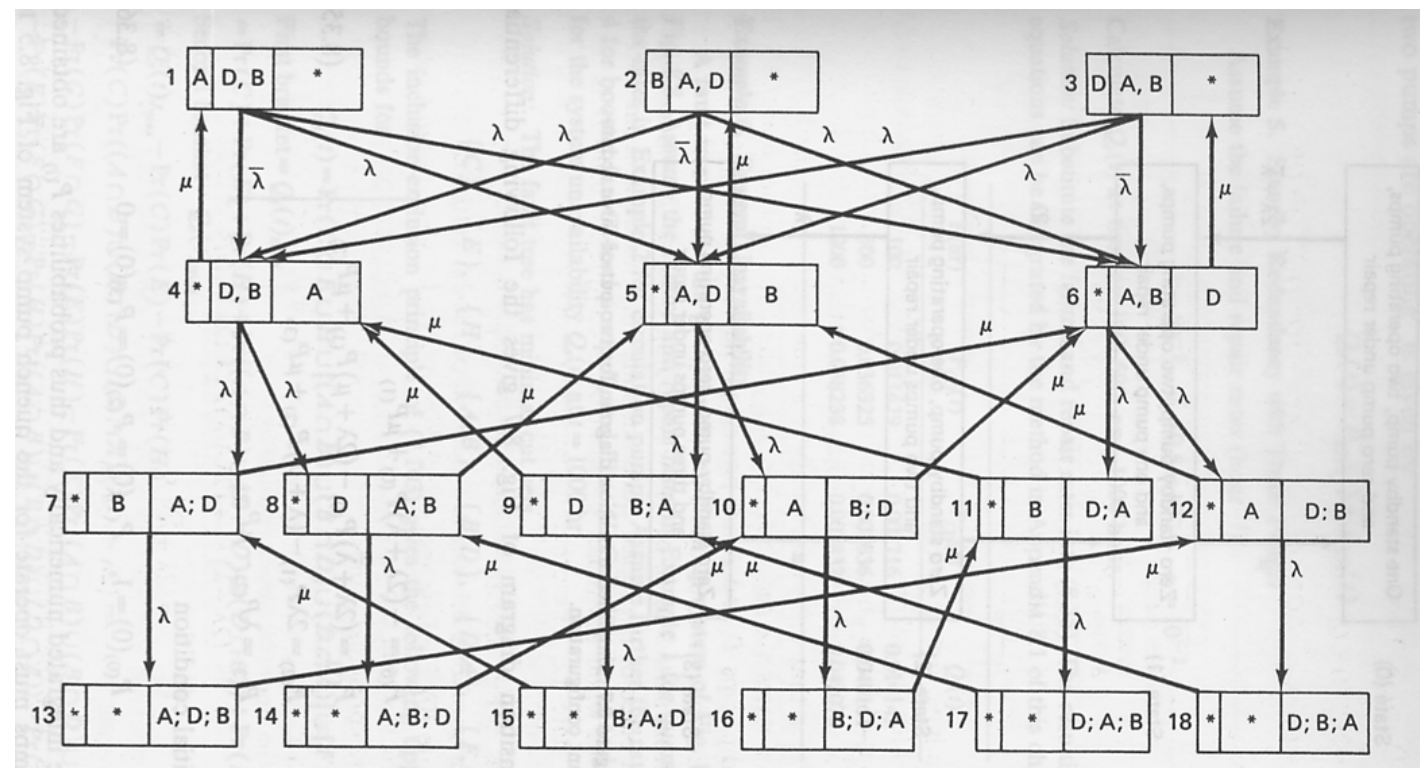

Figure 15: Markov Analysis of a 2 out of 3 System with Warm Spares and Repair [21].

As this figure demonstrates, the diagram for a Markov analysis becomes very complicated even with a relatively simple system. One resolution to this problem is to use a Markov analysis for parts of the system that are dependant and then use the resulting subsystem failure probability as an input into a model which can handle larger systems such as a FTA or RBD.

\subsubsection{PETRI NETS}

A Petri net is a reliability tool first proposed by C. A. Petri in 1962. A Petri net provides the means to analyze the dynamic behavior of systems. A Petri net has two types of nodes: a place node and a transition node. In the Petri diagram, arcs connect the places and transition nodes. One or more tokens can exist in each place marking the state of the system. A transition occurs when every input to that transition has at least one token in it. This "firing" occurs by removing one token from the input and placing that 
token into the output. An example of a Petri net for a repairable system is given as Figure 16.

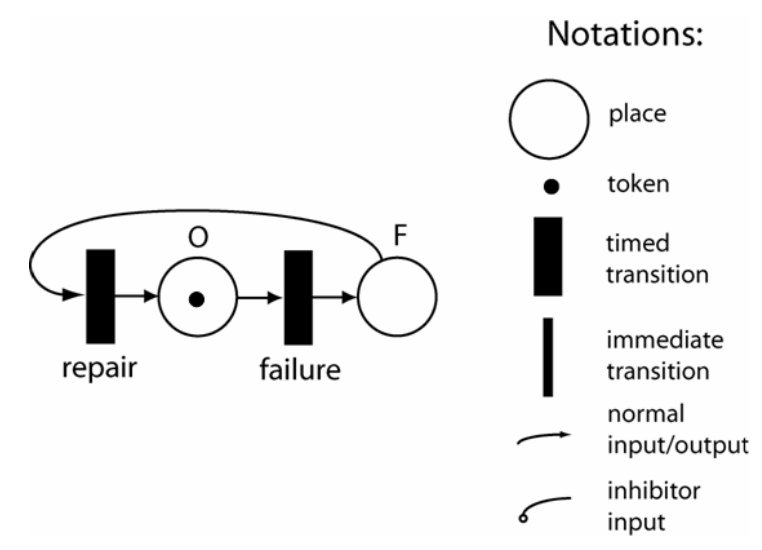

Figure 16: Reparable Unit Petri Net [17].

Petri nets can be used in much the same way as FTA. An example of a generic FTA and the corresponding Petri nets are given below as Figure 17.

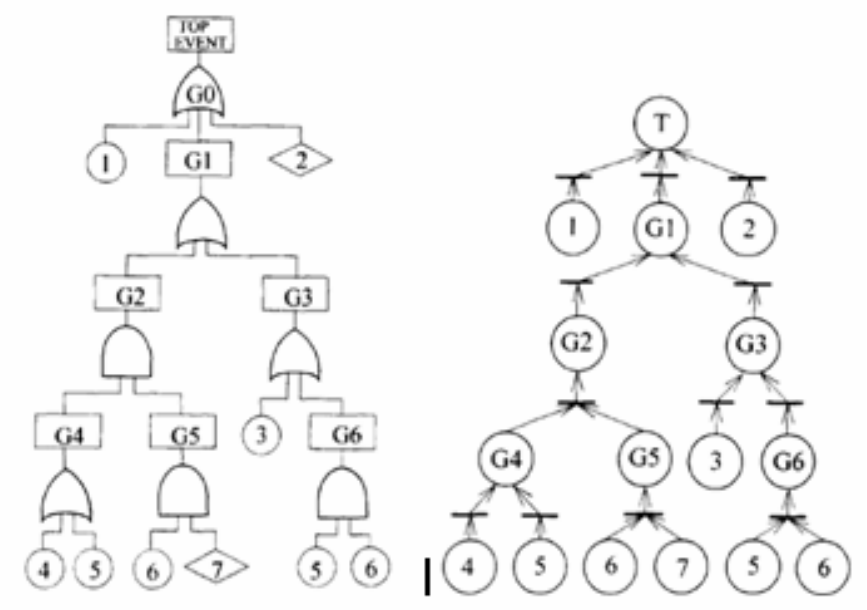

Figure 17: Petri Net of Generic FTA [34]. 
As this figure shows, Petri nets can be used to replicate the work of an FTA. Petri nets are much more powerful in determining reliability because they can model dynamic systems. Petri nets can be used to represent the states of many components over time by monitoring the tokens in the system.

\subsubsection{PROBABILISTIC Risk ASSESSMENT}

Probabilistic Risk Assessment (PRA) is a comprehensive analysis method for analyzing the risk associated with complex technological systems such as aerospace systems. A PRA can include many of the reliability techniques described in previous sections. PRA was first used in the nuclear industry to address the risks associated with nuclear power plants. As noted in section 2.3.2, PRA was first used in the aerospace industry to analyze the risk associated with the Space Shuttle program after the Challenger accident. PRA is so widely accepted at NASA that the agency intends to use PRA in all of its programs and projects to support optimal management decisions for the improvement of safety and program performance [23].

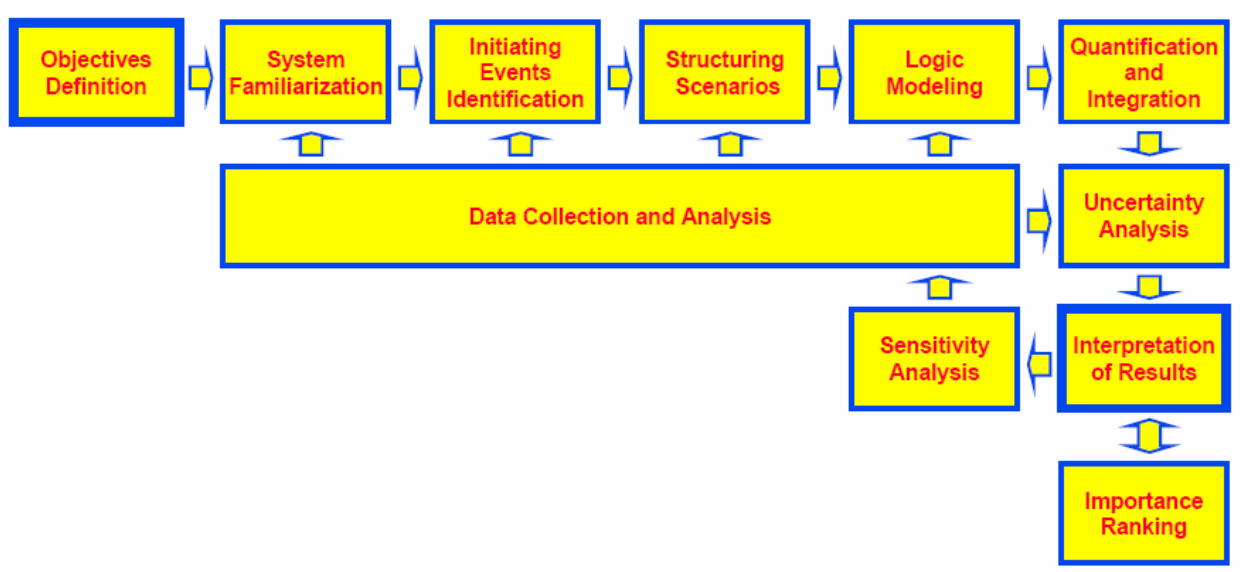

Figure 18: Typical PRA Operational Outline [23]. 
The PRA process begins with the definition of the objectives. This process involves defining possible end states of the assessment. These end states can be failures that result in either loss of mission, vehicle, or crew. The next step is to become familiar with the system under analysis. This is necessary to discover all of the various nuances of the vehicle in question. This can involve a complicated inspection of a current vehicle or an in-depth design analysis for a conceptual vehicle. The third step is to identify the accident scenarios that will lead to the end state defined in the first step. This can be a complicated process since all of the accident scenarios may not have been proposed in the initial design process. The fourth step is to model each scenario in a tool called an event tree. An event tree organizes each accident scenario into the different events that can result from the initial failure. A typical event tree is given as Figure 19.

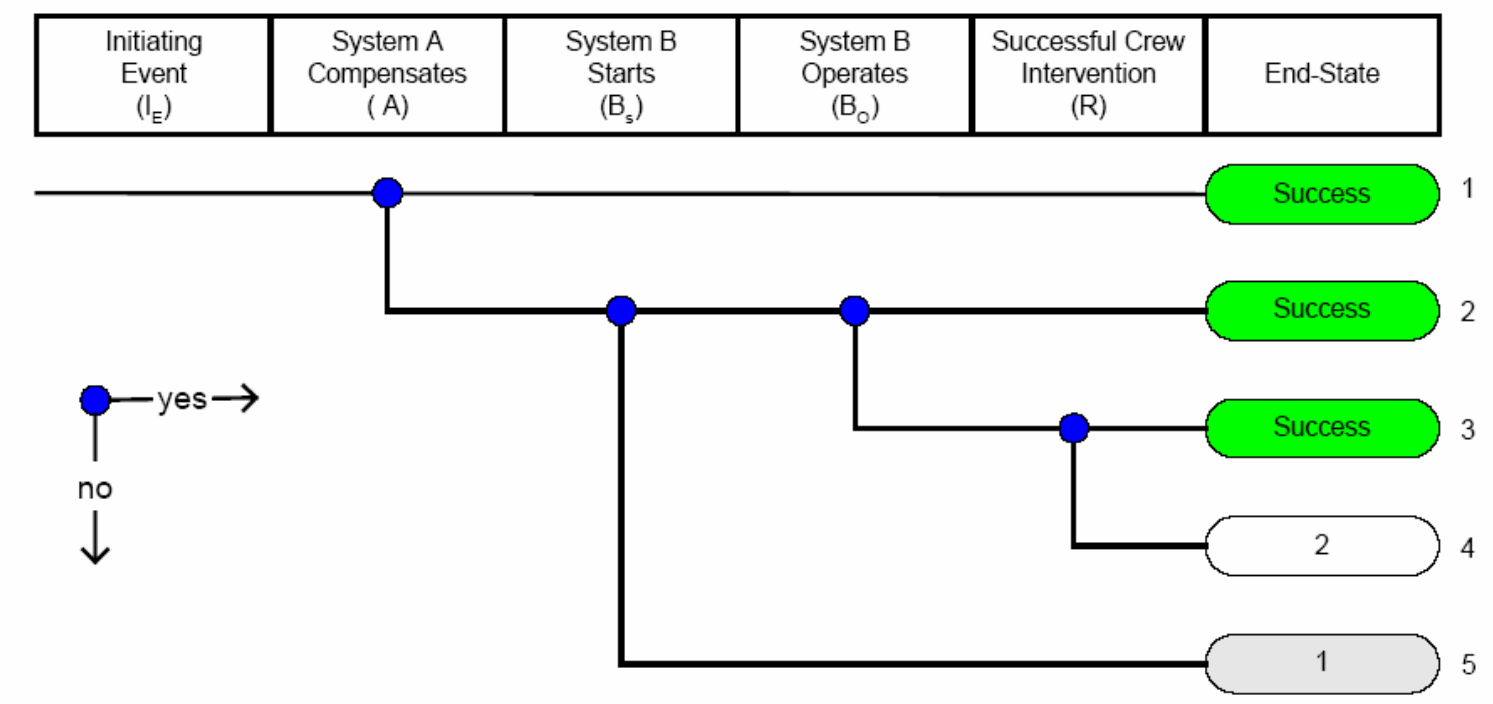

Figure 19: Event Tree Structure [23]. 
The fifth step in the PRA process is to model each accident scenario in a FTA as described in section 2.4.3. Once the FTA is arranged, the next step is to collect the necessary reliability data on the components in the FTA. This can involve failure rate data, repair times, etc. The seventh step is to integrate scenarios that appear in the path of each accident scenario. This is an important step because the components of the FTA must be completely independent. The next step is to apply an uncertainty analysis to the probabilities collected in the previous step. The Monte Carlo analysis used at this stage is described in detail in section 2.6.6. A sensitivity analysis can also be conducted to determine the impact of the different probability inputs to the PRA. The final step is to rank the main contributors to the undesirable end state defined in the first step.

PRA results in an organized collection of the risks associated with a complex vehicle design. PRA is an ideal solution to the risk problem because it is a methodical approach to analyzing all of the different risk factors that could face a vehicle. PRA builds on the existing techniques of reliability engineering and applies the techniques to a complex system. It includes an uncertainty analysis to accurately depict the probabilities of failure due to the different branches of the FTA. It results in a quantitative estimate of the risks as well as potential risk reduction measures that can be taken by the designers.

For this research an dynamically changing version of the FTA is used. Fault trees were chosen over the other reliability techniques presented due to the common use of FTAs in aerospace design. As presented earlier in this dissertation, FTAs were used in the STS program as well as in the ESAS results. Because of this common use and the availability of subsystem reliability data a FTA will be used as the baseline reliability tool. 


\subsection{Cost Estimation TECHNIQUES}

This section will describe the relevant techniques of cost estimation and their application to the conceptual design process. There are a many varying approaches to cost estimation; and, as a result, slightly different resulting costs can be obtained for the same design. The origins of parametric cost estimation began in World War II. At this time there were many aircraft were developed for the war effort, but there was no accurate way to estimate the cost of development of these vehicles. The process has developed significantly as the decades progressed and the design process for aerospace vehicles became more defined. Today, there are a series of organized government lead activities to standardize the cost estimation process. These activities include the Parametric Cost Estimating Handbook sponsored by the Department of Defense (DoD), NASA/Air Force Cost Model (NAFCOM), and the Transcost workbook. These programs were attempts to standardize the cost estimating process to compare competing vehicles on equal footing. A summarization of the costing techniques follows.

\subsubsection{COST ESTIMATION OVERVIEW}

For conceptual design, cost is usually broken down into two groups: recurring and non-recurring costs. Non-recurring costs are the "one-time" costs, such as the cost of vehicle design and the purchase of all reusable hardware. Recurring costs are the costs to operate the vehicle including the purchase of any expendable hardware. In the aerospace community non-recurring costs are generally grouped together as the Design, Development, Testing, and Evaluation (DDT\&E) costs. The DDT\&E is the cost of taking a conceptual vehicle design from the drawing boards to certification for flight. This 
includes the cost to complete the design process, develop manufacturing tooling, produce the subsystem testing, and integration into the entire system.

Recurring costs are generally broken down into the Theoretical First Unit (TFU) cost, the cost of subsequent hardware, and operations cost. The TFU cost is the cost to build the first vehicle resulting from the design process. TFU can be either considered a recurring cost if the vehicle is expendable or a non-recurring cost if the vehicle is reusable. This cost tends to be significantly higher than the average cost for the vehicle since a learning curve generally exists for the production of space vehicles. A learning curve is a type of statistical estimation first suggested by T. P. Wright [35]. In Wright's work he observed that as the process for producing airplanes matured the cost to produce each plane dropped. He proposed a mathematical model to describe this phenomenon.

$$
Y=a X^{\frac{\log b}{\log 2}}
$$

Where: $\mathrm{Y}=$ the cumulative average cost per unit $\mathrm{X}=$ the cumulative number of units produced $a=$ the cost of the first unit (TFU) $\mathrm{b}=$ learning rate (in percentage)

This learning curve is still used today to accurately define the production costs of hardware over the life of a vehicle. Typical learning rates are between $70-100 \%$, with $100 \%$ representing no learning curve. Aerospace vehicle production typically uses a learning curve rate of $85-95 \%$ since these vehicles are complicated systems with low production runs. 
The operations cost is the cost to fly the vehicle. This can be broken down into mission operations and ground operations. Mission operations include the personnel to manage and fly the vehicle, as well as fuel and other consumables, while ground operations includes all of the personnel and equipment necessary to service the vehicle and prepare it for flight. These costs are typically represented as man-hours necessary to complete a mission.

One way to accomplish the cost estimation of an aerospace design, a bottoms-up parametric cost model is used. These parametric cost models are mathematical representations of the correlation between the design variables (typically weight) and the cost of a subsystem. A typical Cost Estimating Relationship (CER) contains algebraic constants which calculate the cost based upon the weight of the subsystem. These CERs are also affected by complexity factors which change the CER based upon the design differences between the analog system and the current design. A generic weight based CER is given below.

$$
\text { Cost }=C F * a(W)^{b}
$$

Where: $\mathrm{a}$ and $\mathrm{b}$ are constants defined by the analogous system

$\mathrm{CF}$ is the complexity factor associated with the design (typically 0.1-2)

$\mathrm{W}$ is the weight of the subsystem analyzed

CERs are useful tools in the parametric cost estimation of a vehicle design. One of the main advantages of the use of CERs is that they can quickly estimate the costs for a changing vehicle design. Because of their mathematical nature, CERs can be quickly 
integrated into a design code and utilized automatically. They can also produce reliable and repeatable estimates if the CERs were constructed from appropriate analog systems to the design being evaluated. CERs have some major weaknesses that were enumerated by the handbook [36]:

1. CERs are sometimes too simplistic to forecast costs. Generally, if one has detailed information, the detail may be reliably used for estimates. If available, another estimating approach may be selected rather than a CER.

2. Problems with the database may mean that a particular CER should not be used. While the analyst developing a CER should validate that CER, it is the responsibility of any user to validate the CER by reviewing the source documentation. Read what the CER is supposed to estimate, what data were used to build that CER, how old the data are, how they were normalized, etc. A user must never use a cost model without reviewing its source documentation.

A parametric cost model is advantageous because it provides a repeatable relationship between input variables and resultant costs. Therefore, aerospace systems that are analyzed by the same cost model can be directly compared to decide the lowest cost system.

A second, more complicated method of cost estimation is the grass roots approach of cost estimation. This method of estimating costs involves an estimation of the work force necessary to construct and operate the design. With this method it is more difficult 
to evaluate the different design changes and the resulting cost of those design changes. For this reason the NAFCOM weight based cost estimation method will be used.

In a cost centric design, the goal of the aerospace designer is to compare the life cycle costs of systems to determine the most cost effective system. Life cycle costs (LCC) incorporates all the costs of the system, including design, production, operation, support, and disposal. This cost includes DDT\&E, TFU, production, and operations cost and is the best discriminator for deciding on the most cost effective system. This analysis typically includes the investment profile of the project, as well as the depreciation of the vehicle and the time value of money. Unfortunately, as described in the problem statement, these costs are usually only considered at the end of the design cycle. This research proposes to calculate these costs up front to decide on the most appropriate lunar architecture based upon the available budget and resulting reliability.

\subsubsection{Department of Defense Parametric Cost Estimating Handbook}

In the fall of 1995 the Department of Defense (DoD) created a joint initiative between both government and industry to standardize the cost estimating procedures used for government procurement programs. A combination of professional and industry cost estimating associations have come together to create this estimating procedure. This group included representatives from the International Society of Parametric Analyst (ISPA), Society of Cost Estimating and Analysis (SCEA), the Space Systems Cost Analysis Group (SSCAG), the Defense Contract Management Command (DCMC), and the Defense Contract Audit Agency (DCAA). This group concluded there were no barriers to the use of parametric cost estimation in both DoD and NASA proposals. A 
summary of the parametric cost estimating procedures recommended by the handbook is given as Figure 20.

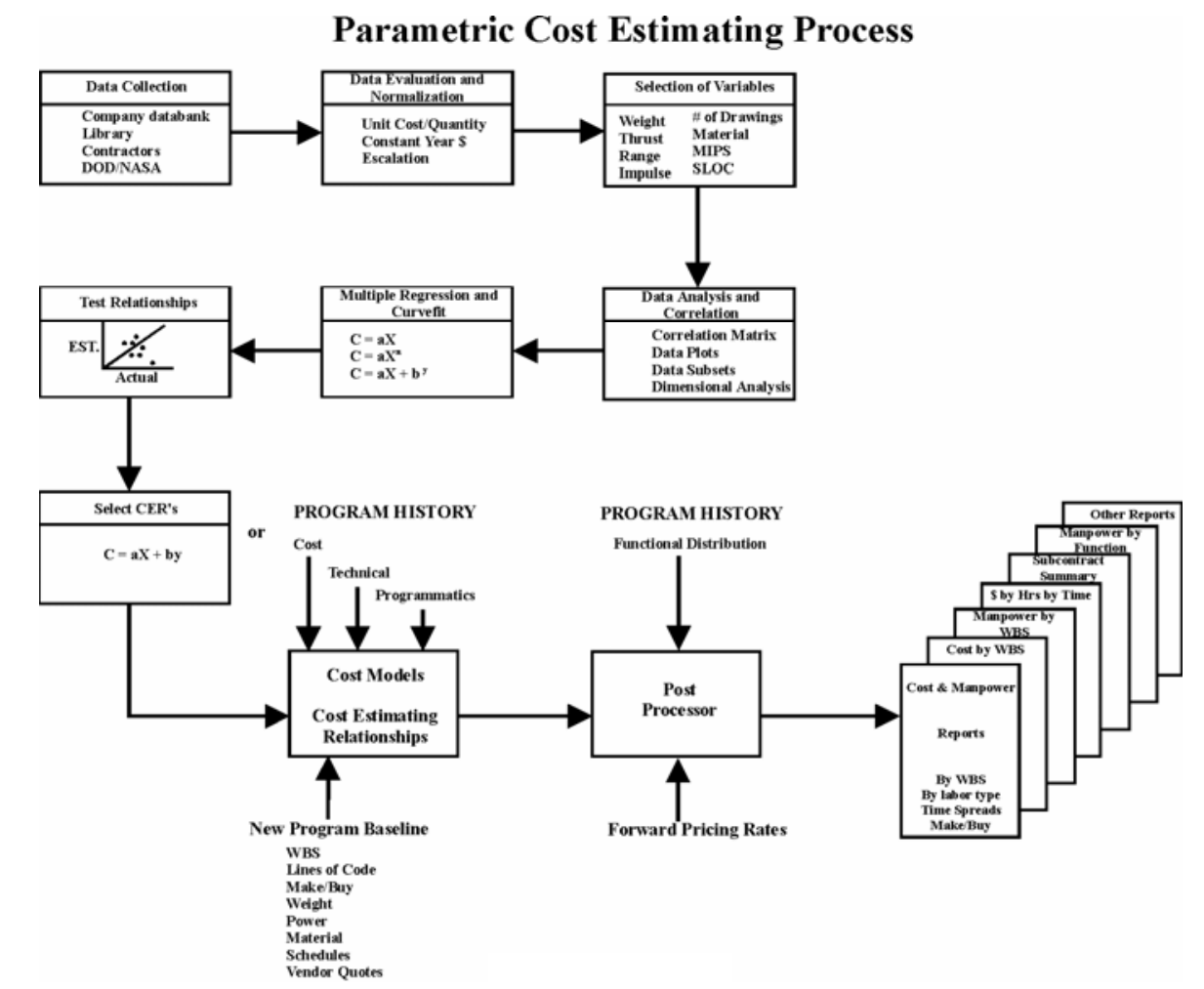

Figure 20: Parametric Cost Estimating Process [36].

As this figure shows, the cost estimation process begins with data collection and evaluation. The data is first analyzed and then plotted. These plots typically contain the cost of various subsystems of existing projects on the dependent axis and a design variable, such as subsystem weight, on the independent axis. From these data points, a statistical analysis can be used to fit a curve to the data, and the CERs can be created. These CERs can then be combined with the parametric modeling tools to produce the vehicle costs. These costs should then be rationalized with previous programs to verify their validity. 


\subsubsection{NASA AIR FORCE COST MODEL (NAFCOM)}

The NASA Air Force Cost Model (NAFCOM) is a costing tool developed to model both the DDT\&E and the hardware costs for aerospace vehicles. NAFCOM is generally accepted as the most appropriate parametric cost model for the evaluation of the costs of a single vehicle [37]. The NAFCOM tool is a software database that has historical subsystem costs for a variety of aerospace projects. The data is broken down by subsystem and mission type and is then normalized to produce accurate cost estimates for new vehicle designs. NAFCOM facilitates a bottoms-up approach to cost estimation by estimating the costs of each subsystem using CERs of analogous systems.

NAFCOM was developed by the Science Applications International Corporation (SAIC) to provide a parametric cost estimating tool for space hardware. Since the early nineties, eight versions of NAFCOM have been developed, with the most recent being created in 2004. NAFCOM builds upon the Resource Data Storage and Retrieval (REDSTAR) library which contains aerospace project data from the early sixties. NAFCOM has since been consolidated into a stand alone software tool complete with graphical user interfaces to allow easy implementation in the cost analysis of a vehicle design.

\subsubsection{TRANSCOST COST ESTIMATING HANDBOOK}

The Transcost cost estimating handbook is similar to the NAFCOM software tool in that it is a repository for CERs for aerospace vehicles. The Transcost handbook was first compiled in 1971 and has been updated until the latest version published in 2000. The Transcost handbook contains data from US, European, and Japanese space vehicles dating from 1960-2000 [38]. The Transcost is a database of top-down CERs for space 
launch vehicles. It is intended for use as a system model to compare the costs of different systems at the top level. This process is advantageous at the conceptual vehicle design level because the details of the subsystems may not be completely defined. The Transcost model was defined to choose the most economic vehicle concept from a list of alternatives early on in the design process. A summary of where Transcost fits into the vehicle design process is shown below as Figure 21.

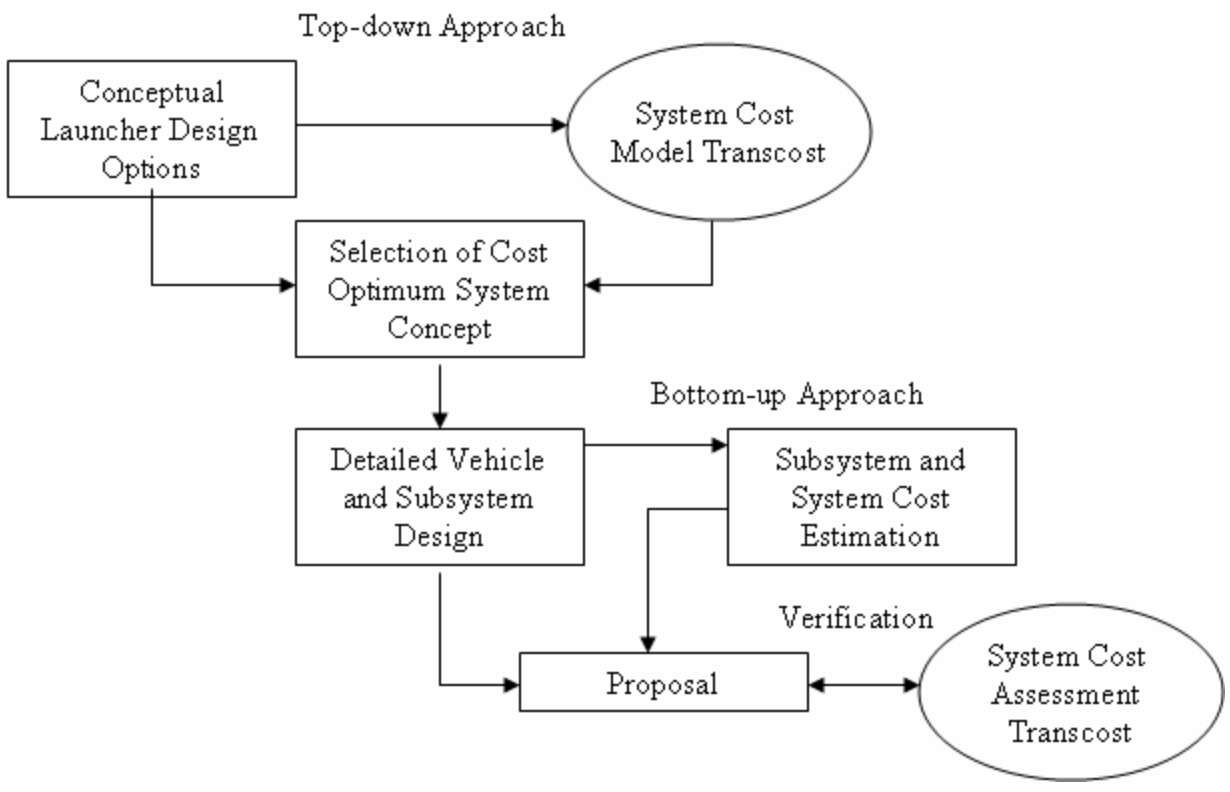

Figure 21: Cost Estimation via Transcost [38].

As this figure shows, the Transcost methodology is designed to use CERs at the top level to select the proper design. Once this design is selected, a detailed analysis is conducted using a bottoms-up approach, like the NAFCOM cost model. Transcost can then be used again on the more refined system to verify the results of the bottoms-up approach. 


\subsection{Current Conceptual Vehicle Design Process}

This section will outline the current conceptual vehicle design process used at the Space Systems Design Laboratory at the Georgia Institute of Technology. The conceptual design process involves a combination of multiple design disciplines. These disciplines are treated as individual contributing analyses to the entire vehicle design. Each of these contributing analyses is coupled, which makes a difficult design problem. This coupling requires iteration between the disciplines to close the vehicle design.

Once this analysis is defined, there are many different methods to optimize the design. These are all grouped under the topic of Multidisciplinary Design Optimization (MDO). For very complicated designs, an MDO process, which directly runs the contributing analyses, may not provide the necessary efficiency. In these cases, a Response Surface Methodology (RSM) can be used to approximate the design disciplines. Once the MDO process has been completed, closing a particular design, a ROSETTA model can be made. ROSETTA models utilize RSMs to allow the designer to quickly trade the design parameters of a vehicle. Once this fast acting tool is created, an uncertainty analysis can be conducted using Monte Carlo Simulations. An introduction to each of these topics is included in the following section.

\subsubsection{ConCEPTUAL DESIGN DisCIPLINES AND THE DESIGN STRUCTURE MatRIX}

As described in the introduction to this section there are many different coupled disciplines that make up a conceptual vehicle design. This coupling between the disciplines is graphically represented as a design structure matrix. Each of the contributing analyses (design disciplines) is represented as a box in the DSM; the links between the boxes are the coupling variables that are passed between the disciplines. 
Links leaving the right hand side of the boxes represent data that is passed downstream to the following design disciplines. The links leaving the left hand side represent information that is required upstream in the design process. This information typically includes design variables that are approximated in the analyses preceding the active discipline, which will now be rerun with the correct values of the variables. This procedure must then be iterated so that the design disciplines are all using conforming values for the design variables.

The DSM for a typical launch vehicle design involves two different iteration loops which were described in section 1.2.1. For this generic launch vehicle design, the first iteration loop is between the propulsion, trajectory, aeroheating, and weights and sizing disciplines. This iteration loop closes the performance aspects of the vehicle. The second iteration loop is between operations, reliability, and cost. This loop uses the converged physical design and creates the operations, reliability, and costs of the closed design. A typical DSM for a launch vehicle is given as Figure 22.

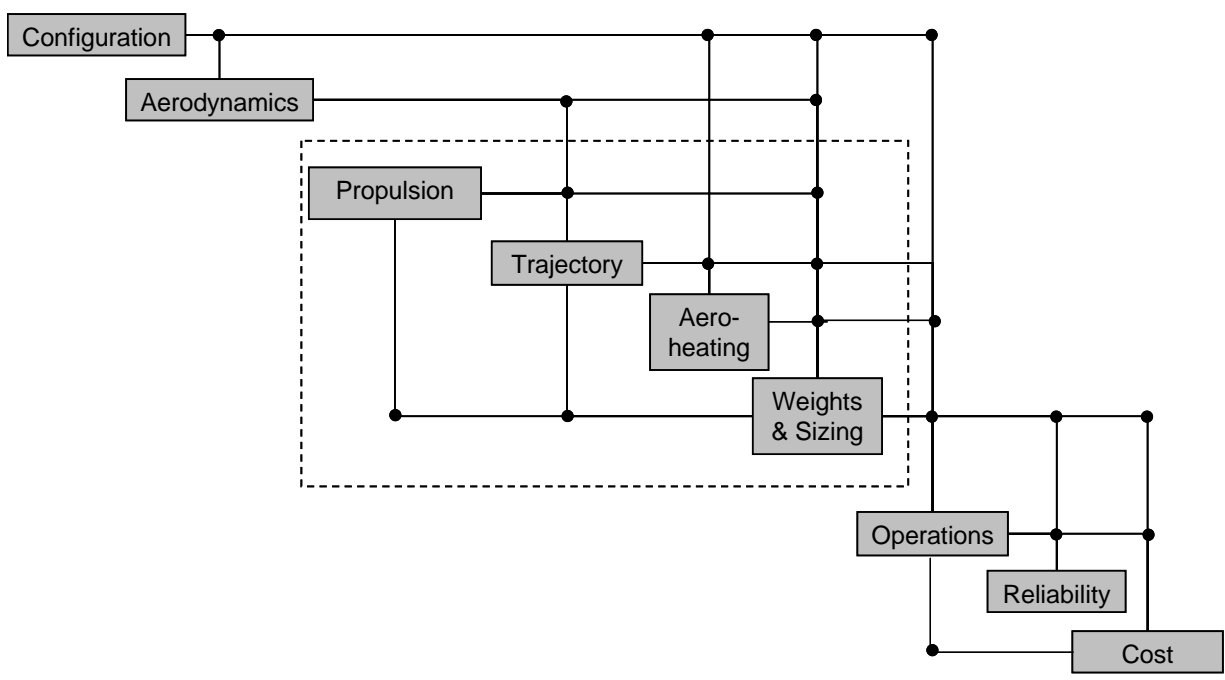

Figure 22: Design Structure Matrix for a Generic Launch Vehicle. 
Each discipline in the DSM is associated with one or more conceptual design tools associated with it. Table 6 provides a listing of each discipline and a corresponding typically used aerospace design tool or tools. Configuration, aerodynamics, propulsion, trajectory, structural design, aeroheating, and reliability are all analyzed with their respective disciplinary tool. Weights and sizing is typically composed of a series of Mass Estimating Relationships (MERs) that are summarized and internally closed in an MS Excel workbook. Cost CERs are based upon parametric costing analyses as described in detail in section 2.5.

Table 6: Typical Design Tools for Conceptual Vehicle Design

\begin{tabular}{cc}
\hline Discipline & Analysis Tool \\
Configuration & Computer Aided Design (CAD) \\
Aerodynamics & APAS (HABP) \\
Propulsion Design & REDTOP \\
Trajectory & POST 3-D \\
Weights \& Sizing & MERs \\
Structures & GT-STRESS \\
Aeroheating & Miniver \\
Reliability & Relex \\
Cost & TRANSCOST \\
\hline
\end{tabular}

This table only provides a sampling of some of the reference tools implemented in the contributing analyses associated with a conceptual vehicle design. For example, POST 3$\mathrm{D}$ is a typical trajectory tool for the conceptual design of a launch vehicle. Other in-space trajectory tools may be more appropriate for the interplanetary missions. Each of the design disciplines in the DSM are analyzed and then iterated to find the closed solution to 
the vehicle design. A summary of the different design disciplines used for the launch vehicle described in Figure 22 follows:

\section{Internal Configuration:}

This design discipline is usually determined via geometric relationships and computer aided design codes. This discipline is necessary to determine the tank and structural layouts of the launch vehicle. It is also necessary to determine the interactions of the stages and the engine placements. Although this discipline technically changes with the changing size of the vehicle, for most models it is considered to change photographically with the size of the vehicle. For simpler vehicles, configuration is not necessary because the tank sizes and structural sizes can be calculated via simple geometric relationships and therefore is combined with the weights analysis.

\section{Aerodynamics:}

This design discipline is necessary to determine the aerodynamic coefficients of the vehicle during the atmospheric flight portion of the trajectory. The lift and drag coefficients were calculated via an aerodynamics simulation code. For most vehicle models, Aerodynamic Preliminary Analysis System II (APAS) was used to calculate these coefficients [39]. These aerodynamic coefficients were then used with the scaled cross sectional area to find the total aerodynamic forces on the vehicle.

\section{Propulsion:}

This design discipline is necessary to determine the engine performance of the vehicle during the powered portion of the trajectory. To calculate the engine performance, REDTOP, a conceptual chemical rocket design code was used [53]. 
REDTOP requires that the engine cycle type, propellant type, and thrust level are given from the architecture design. REDTOP then calculates the $I_{\mathrm{sp}}$, and engine thrust to weight which is necessary to calculate the weight and trajectory of the vehicle. This design tool is a stand alone program which must be run in every iteration of the DSM to calculate the engine performance.

\section{Trajectory:}

Much like the Propulsion discipline, the trajectory discipline is in the iteration loop of the vehicle. The trajectory analysis is also completed by a conceptual design tool which must be completed each iteration of the design process. This trajectory discipline is optimized using a three degree of freedom trajectory simulation code called the Program to Optimize Simulated Trajectories (POST 3-D) [55]. This trajectory simulator iterates through the equations of motion to find the ideal trajectory for a given vehicle configuration. For most vehicle model, the payload was treated as a constraint, with the optimizer set to minimize fuel consumption.

\section{Mass Estimation Relationships:}

Much like the Propulsion and Trajectory disciplines, the mass estimating discipline is in the iteration loop of the vehicle. This discipline uses Mass Estimating Relationships (MERs) to calculate the dry weight of the vehicle as a function of the total propellant required. This dry weight is a function of the engine thrust to weight from the propulsion discipline, the propellant calculated from the trajectory analysis, and the configuration inputs. A summary of MER database used in this analysis is given as [57]. 
The Reliability and Cost disciplines are calculated using new derived methods of the NAFCOM cost model and the FTA analysis. NAFCOM was used as the costing model because of its accuracy for aerospace vehicles including those used in a lunar architecture. An FTA analysis was chosen over other reliability analyses due to the availability of subsystem data and the prevalence of FTA analysis in aerospace design. A summary of the changes to both the NAFCOM cost model and the FTA reliability analysis is given in Chapter 3 .

To achieve a closed design, the contributing analyses are iterated until the inputs to the individual modules do not change. This iteration is necessary because of the feedback loops in the DSM. Once the vehicle is closed each of the CAs are using consistent data and an actual conceptual design is completed.

\subsubsection{MULTIDISCIPLINARY DESIGN OPTIMIZATION}

Once the DSM has been successfully constructed, a method must be used to close the vehicle design. The disciplines within the DSM compete for the available resources as well as the selection design parameter values, to produce the ideal solution. Historically, a Fixed Point Iteration (FPI) method has been used to close the vehicle design. This method iterates among the design disciplines to determine a closed solution. Unfortunately, this solution may not be ideal. To solve this complex problem Multidisciplinary Design Optimization (MDO) techniques were created. MDO is series of techniques to find the optimal solution for a complex engineering problem consisting of many conflicting design disciplines. A summary of the disciplines used in a conceptual launch vehicle design is given in Table 7. 
Table 7: Characteristics of Design Disciplines for a Conceptual Launch Vehicle [40].

\begin{tabular}{ccc}
\hline Discipline: & Variable/Constraint Number & Code Type: \\
and Type: & & \\
Geometry/packaging & Few-to-many variable, & Interactive, graphical, \\
& continuous/discrete & commercial \\
Aerodynamics & Few variables, continuous & Interactive \\
Trajectory & Many, continuous, & dense/sparse \\
& Fesign-oriented \\
Weights and Sizing & continuous/discrete & Design-oriented \\
& Few, continuous/discrete & Interactive \\
Heating & Many, continuous/discrete & Batch, commercial \\
Structures & Many, continuous & Batch, commercial \\
Controls & Few, continuous/discrete & Design-oriented, \\
Propulsion & & regression equations \\
& Many, discrete & Highly interactive, \\
Operations & & regression equations \\
& Highly interactive, \\
Cost & Many, continuous/discrete & regression equations \\
\hline
\end{tabular}

As this table demonstrates each design discipline uses different types of variables as well as multiple different analysis codes. This makes the MDO problem very difficult since one optimization scheme will not address all of the different design problems faced by the individual disciplines.

There are many different MDO methods available to the aerospace designer. A short list includes the All at Once Approach (AAO) [41], Collaborative Optimization (CO) [42], and Bi-Level Integrated System Synthesis (BLISS) [43]. These MDO techniques have been used repeatedly in the design of aerospace vehicles. There is a wealth of literature on their implementation and effectiveness as it pertains to the aerospace design problem. A comparison of the techniques for a reusable launch vehicle 
was conducted by Brown [41] at the SSDL at Georgia Tech using the same design process outlined in this section. His qualitative results are included as Table 8.

Table 8: Comparison of MDO Techniques for a Conceptual Launch Vehicle [41].

\begin{tabular}{lrccc}
\hline Criteria & FPI & $A A O$ & $B L I S S$ & $C O$ \\
Implementation Difficulty & A & C & B + & C+ \\
Total Execution Time & A & A- & B + & B- \\
Model Robustness & A & D & B + & B \\
Formulation Difficulty & A & A & B + & B- \\
Optimization Deftness & D & A- & A & B \\
Convergence Error & A & A- & B + & C + \\
\hline
\end{tabular}

As evidenced by this table, all of the MDO methods presented were able to complete the reusable launch vehicle problem with differing levels of success. Although each method has advantages and disadvantages, most arrived at the ideal solution much faster than a traditional manual process.

\subsubsection{MultidisCiplinary DESIGN OPTIMIZATION With GENETIC AlgoRIthMS}

A genetic algorithm is a stochastic, domain spanning method to solve complicated optimization problems. The optimization process mimics that of nature's natural selection process. Several generations of design variables are conducted and the best designs survive to pass their characteristics on to the next generation. After several generations the best designs should be found through the natural selection.

Genetic algorithms begin by initializing an initial population of designs. This initial population is created randomly from the discretization of the design variables 
through the use of a binary string. The discretization is dependent on the range of the design variables and the number of bits designated to each variable. The step size of the discretization is determined by equation 4 .

$$
\text { StepSize }=\frac{\Delta x}{2^{n}-1}
$$

Where: $\mathrm{n}$ is the number of bits in each design variable $\Delta \mathrm{x}$ is the range of the variables.

Therefore as $n$ increases, the step size decreases, and the total number of available points increases.

Once the design variables are discretized an initial population is randomly created. This initial population can vary in size, but for this paper the population size is given by equation 5 .

$$
\text { popsize }=3 * n * N
$$

Where: $\mathrm{n}$ is the number of bits in each design variable

$\mathrm{N}$ is the number of dimensions of the problem.

Once this initial population is instantiated the three biologically inspired processes are implemented on the population to create the next generation.

The first biologically inspired process is reproduction. Reproduction is the start of a new generation through a survival of the fittest type selection of the initial 
population. There are two main methods to accomplish reproduction. The first is proportional replication. In this process each design in the initial population is evaluated. Each is then proportionally compared to the fitness $(1 / \mathrm{F})$ of the entire population (equation 6). The number of times that design appears in the next population is then given by equation 7 .

$$
\begin{aligned}
P_{i} & =\frac{f_{i}}{\sum f_{i}} \quad \text { where: } \quad f_{i}=\frac{1}{F\left(x_{i}\right)} \\
N_{i} & =\operatorname{round}\left[\operatorname{popsize}^{*} P_{i}\right]
\end{aligned}
$$

The second method of reproduction is that of a tournament selection. Tournament selection randomly selects two "combatants" from the initial population. The one with the lower function value is determined the winner of the battle and is promoted to the next generation. This is then repeated until the population size is reached. Of the two methods proportional replication always forces the best designs into the subsequent population, unfortunately it is ill posed for a problem where $\mathrm{F}$ is less than or equal to 0 . The tournament method is more robust since there are no restrictions of F, yet there is a possibility for poor designs to propagate into further generations due to the randomness of the combatants. It should be noted that this is the only process where it is necessary to evaluate the function. Therefore the method chosen and the number of generations, determine the number of function calls for the entire program. For proportional replication there is exactly one function call per member of the population per generation. For tournament selection there are many methods to evaluate the function. In this 
instantiation of the tournament selection each time a combatant is chosen randomly the function value for that design is calculated. Therefore there are two calls per population size, per generation. This can be reduced through an evaluation of the initial population put into memory and the random number generator choosing from that array. Also since reproduction is where the designs are evaluated this is also where the best designs for a given generation are stored.

The second biologically inspired process of GA is that of crossover. Crossover is a way to genetically splice two parent strings in hopes of creating a better design. It is accomplished by randomly picking two parent strings from the post-reproduction population and applying a predetermined probability that the parent's genes undergo a crossover. This probability is usually about seventy percent of the time. After this crossover occurs the parents are eliminated from crossover consideration and the next crossover pair is determined until the entire population is evaluated. As in reproduction there are two different methods to perform a crossover. The first is known as two-point crossover. This involves taking the parents that have been selected for crossing over and applying two random numbers. The first is the bit where the parents will start crossover and the second is the amount of bits that will be crossed. Once this window is created the bits of the parents in this window are switched and returned to the population. The second form of crossover is uniform crossover. This involves going bit by bit in a string and applying the probability of crossover to the bit. The goals of the crossover process are to randomly splice the "good" designs of reproduction in the hopes of finding the best design. 
The third biologically inspired process is mutation. Mutation is the selection of a single bit in a genetic string to be changed. This process introduces some diversity into a population that has essentially been inbreeding in the previous two processes. Mutation allows designs that are outside the initial population to be more readily tested. A typical mutation rate is usually one to two percent. It has been found recently that higher percentages of mutation seem to work better for typical optimization problems so mutation percentages of twenty are typical.

This process can then be repeated for a given number of generations to determine the solution. This is done by storing the best design from each generation and saving it. The best of these best designs is then chosen as the best ever design and the resulting optimum. A diagram of the iteration loop is given as Figure 23.

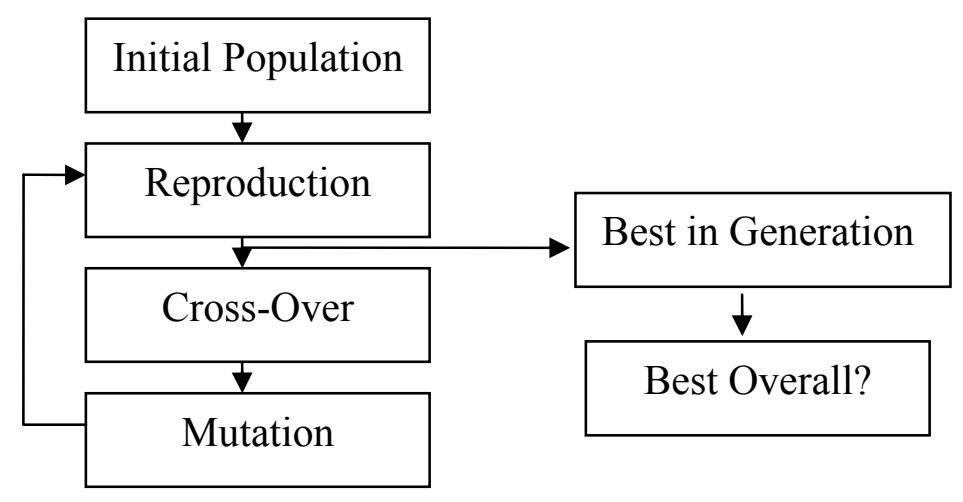

Figure 23: Genetic Algorithm Overview

The natural discritization of the GA process makes it an ideal optimization scheme for problems in which there are a low percentage of continuous variables. This optimization scheme, when combined with a multidisciplinary analysis such as Fixed Point Interation (FPI), is effective at finding ideal solutions for complicated conceptual design problems. 
For the research described in this paper, a GA is used with an Overall Evaluation Criteria (OEC) as the objective function to find the ideal Pareto Frontier for the lunar mission modes. A GA was chosen as the optimization scheme of choice because of the inherent ability of GAs to deal with discrete variables. In a lunar architecture selection problem, over $75 \%$ of the variables are discrete. A GA was chosen over a simple design space exploration because of the ability of the GA to find the ideal solution in less function calls.

\subsubsection{RESPONSE SURFACE METHODOLOGY}

Response Surface Methodology (RSM) is a relatively new method for approximating complex analyses. RSM is a multivariate regression techniques developed to model the response of a complex system using a simple algebraic equation. This response surface equation can then be used in place of the physics based design tool. This saves both time and resources, since the equation can be evaluated quickly and without the involvement of the discipline experts.

The RSM begins by identifying the design variables that the response surface will incorporate and the ranges in which these variables are to vary. This is an important step because the response is only as good as the included variables. If the proper variables are not modeled, the response will be meaningless. The ranges set for the design variables are also critical. If the ranges are set too narrow the response may be good, but the model will be less useful because the response surface equations should only be used within the ranges defined. If the ranges are set too wide the response fit will likely be bad since the regression analysis is trying to fit very diverse data. 
The second step of the RSM is to do a linear screening test to find the important design variables that were set in the first step. A two level Design of Experiments (DOE) is run as a screening test. A DOE is an organized approach to choose which sets of design variables to run so that the responses can be evaluated without the entire spectrum of design variables begin computed. Classical DOEs are comprised of one of five designs: Full Factorial, Latin Hyper Cube, Box-Behnken, Face-centered Central Composite Design (CCD), and D-optimal. Each of these designs uses a combination of the points shown in Figure 24 (full factorial runs the entire design space).
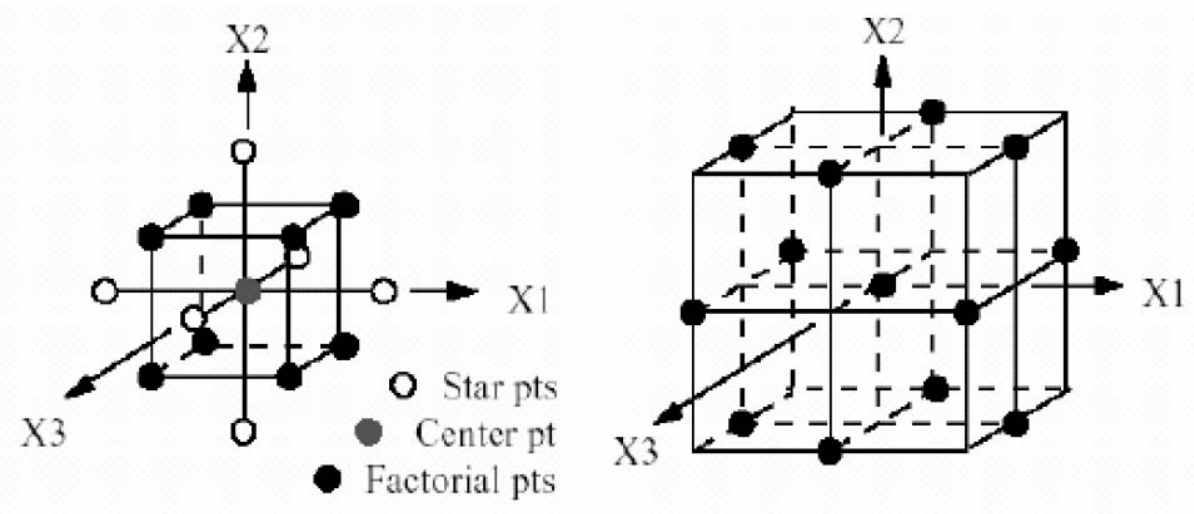

Figure 24: Dimensional Representation of Design of Experiments [44].

Once the model is chosen the design variable combinations are selected and the experiments conducted, a process called Analysis of Variance (ANOVA) is used to select the most appropriate variables for use in the actual response surface. The ANOVA process usually involves a line chart to show which variables have the most significance. 


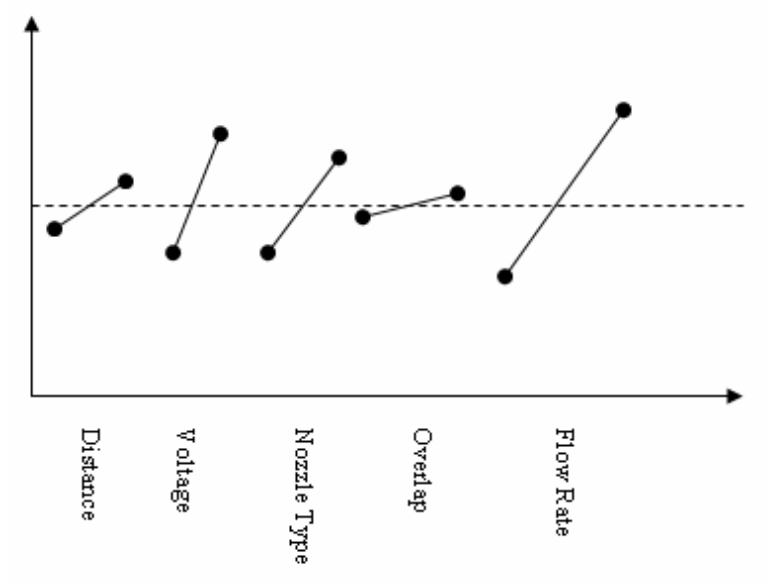

Figure 25: Results of the Screening Test for a Nozzle [45].

Line charts such as Figure 25 are then used to find the variables which have the most significant impact on the design. For this simple example, distance and overlap may be excluded if only three design variables are required.

Once the screening test is complete and the proper design variables and their respective ranges are defined, a three level DOE is completed for the appropriate design variables. This includes fitting the results with a Response Surface Equation (RSE). The typical form of a RSE is given below as equation 8 .

$$
R=b_{0}+\sum_{i=1}^{k} b_{i} x_{i}+\sum_{i=1}^{k} b_{i i} x_{i}^{2}+\sum_{i=1}^{k-1} \sum_{j=i+1}^{k} b_{i j} x_{i} x_{j}+\varepsilon
$$

Where: $k$ is number of variables

$x_{i}$ is the main effects

$x_{i}^{2}$ is the quadratic effects 
$x_{i} x_{j}$ is the second order interaction terms

$b_{0}$ is the intercept term (contains effect of all ignored variables)

$b_{i}$ is the regression coefficients for the first degree terms

$b_{i i}$ is the regression coefficients for the pure quadratic terms

$b_{i j}$ is the regression coefficients for the cross-product terms

$\varepsilon$ is the error associated with neglecting higher order effects

The resulting RSE must then be tested to assure that it is an accurate representation of the physics based model it is approximating. This is done by conducting an analysis of the goodness of the RSE fit. This check usually involves a visual inspection of the Actual points plotted against the RSE's predicted points, an analysis of the $\mathrm{R}^{2}$ of the fit, and a verification test where the actual physics based analysis is compared with the RSE model on design points that were not used to create the RSE. Once verified, the RSE can be used in place of the legacy design code for the ranges specified in the first step. This RSE provides a reasonably accurate approach to finding the optimal design. Once the design is selected, then the chosen set of design variables can be analyzed in the physics based code to verify the results.

\subsubsection{ROSETTA MODELS}

A Reduced Order Simulation for Evaluating Technologies and Transportation Architecture (ROSETTA) model is a parametric design tool utilized by the Space System Design Laboratory at the Georgia Institute of Technology to quickly evaluate how changing the design variable affects a vehicle. A ROSETTA model is a spreadsheet model that uses RSM to approximate each of the design disciplines described in section 2.6.1 [46]. This includes a RSE for the high fidelity tools such as POST, APAS, and GT 
Stress. The ROSETTA model can also be used to calculated the viability of a vehicle if the DDT\&E, operations costs, and economics of the vehicle are included in the model.

The NASA ATLAS project used the ROSETTA model extensively to calculate both lunar and Mars architectures. The ATLAS project was tasked with creating a framework to quantitatively evaluate the influence of different technologies on lunar and Mars architectures. ROSETTA models were created for each of the vehicle types and were integrated into the ATLAS framework via the Generic Model Template (GMT). The ATLAS code then passes the appropriate design variables, including technologies from each of the architecture elements to close the reference mission.

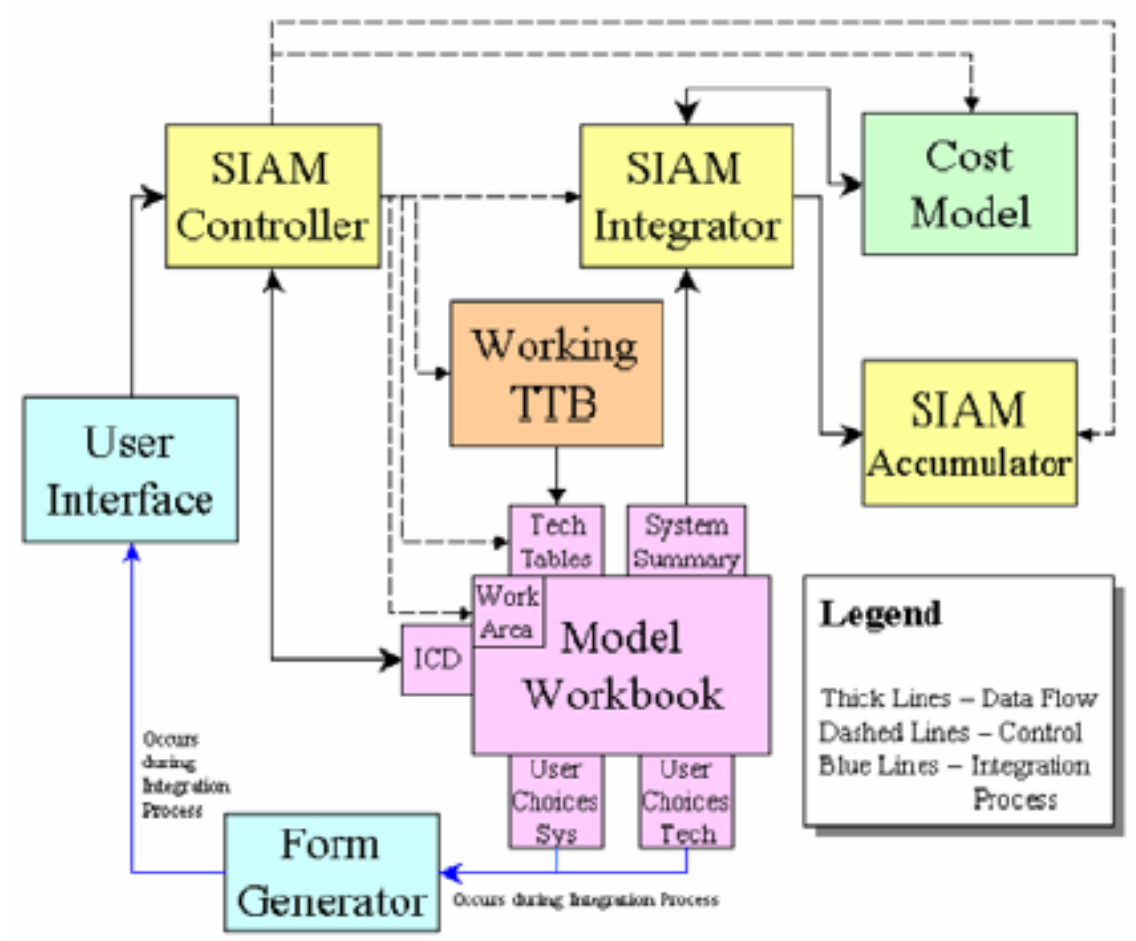

Figure 26: ROSETTA Models in the ATLAS Framework [47]. 
Figure 26 depicts the process flow of the ROSETTA model that was used in the ATLAS framework. The ROSETTA models are denoted in the figure as the model workbooks. As this diagram shows, the ATLAS framework calculated the costs and the technology data separately from the ROSETTA models. The ROSETTA model was ideal for the ATLAS application because the ROSETTA model can parametrically adjust to the changing design variables quickly. The fact that ROSETTA models can accurately and quickly evaluate the design of a vehicle makes uncertainty analysis feasible for an aerospace vehicle design.

ROSETTA models can very in the fidelity contained in the model. For this research, ROSETTA models will be split into three levels. These levels are derived from the ATLAS project and were slightly modified for use in this thesis.

- Level 1- Lowest fidelity model, in which most inputs are static or simple historical relationships. Most performance and cost relationships are qualitative with some quantitative physics based models (such as the modified rocket equation) are included.

- Level 2- Most widely used type of model. Includes historical as well as physics based mass estimating relationships. Initial trajectory and performance points are verified with a conceptual design tool to estimate non ideal parameters, but scaling relationships are used for off nominal points.

- Level 3- Highest fidelity model. Response Surface Equations are used to represent the conceptual design disciplinary tools. Design of Experiments completed for all necessary physics based codes. Trajectory and Engine performance are all simulated via these response surfaces. 
For this research, all of the ROSETTA models will be either level 2 or level 3 models. The fidelity of the model will depend on the complexity of the vehicle modeled. All lunar ascent and descent modules will be level 3 models because of the complexity of the trajectories and the importance of engine performance. Command modules will be level 2 because of the lack or propulsion and fact that all powered trajectories are performed by other vehicles.

ROSETTA models will be used in this research to parametrically evaluate the performance of the vehicles in the analyzed lunar architecture. A ROSETTA model will be created for each vehicle class that will be evaluated for this research. The ROSETTA models will then be combined in an integrated framework with a Genetic Algorithm optimizer to create the Pareto frontier for the costs and reliabilities for each mission mode.

\subsubsection{Monte Carlo Simulations}

In conceptual design, it is important to properly estimate the uncertainty associated with each design decision. This is true for the choices made for the performance variables as well as the programmatic variables associated with a lunar vehicle design. To accomplish an uncertainty analysis on a vehicle design a Monte Carlo simulation is typically used. A Monte Carlo simulation is a technique that simulates large numbers of random events [45]. This simulation is conducted by setting up distributions on the design variables in the ROSETTA model. These distributions assign a probability of choosing a certain value of the design variable, the higher density the distribution the higher the probability of selecting that value. The Monte Carlo 
simulation then runs the ROSETTA model hundreds of thousands of times with different inputs randomly selected via the distributions on the input variables.

There are many different distributions that can be used on the inputs of the Monte Carlo Analysis. For this research, a triangular distribution will be used on all of the subsystem reliabilities. A triangular distribution requires minimum, maximum, and nominal reliabilities to create the distribution. The nominal is the highest probability of occurring and the probability of occurrence decreases linearly on both sides from the maximum nominal value to a zero probability at the minimum and maximum value.

The result of a Monte Carlo analysis are Probability Density Functions (PDFs) for the designated output variables. A PDF is a graphical collection of the response variables. A PDF can then be integrated into a Cumulative Distribution Function (CDF). A CDF allows the user to see what percentage of Monte Carlo runs provided a minimum required response. This process is outlined in Figure 27.

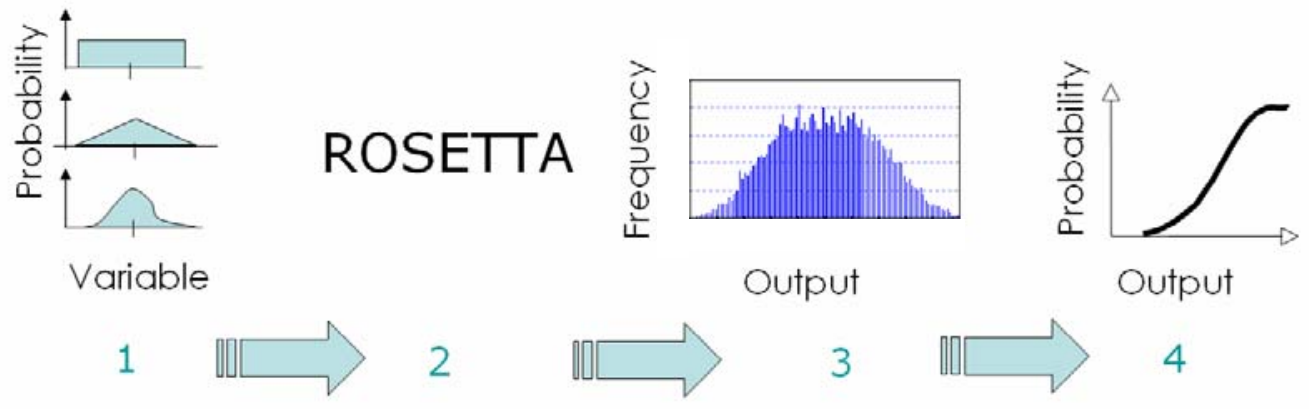

Figure 27: Monte Carlo Simulation Flow Chart.

In this figure, the assignment of distributions to the input variable is step 1. Step 2 is the application of the Monte Carlo analysis to the ROSETTA models. Step 3 is the creation 
of the PDF from the Monte Carlo output. Finally, step 4 is the integration of the PDF to create the $\mathrm{CDF}$. The $\mathrm{CDF}$ is then used to find the probability of reaching a certain performance level.

For this research, a Monte Carlo analysis will be conducted on the input subsystem reliabilities to get a confidence prediction on the resulting vehicle reliabilities and safeties. The resulting $90 \%$ confidence $(90 \%$ of the simulations have lower reliabilities and higher costs) and $10 \%$ confidence bands can then be compared with the baseline to get a better understanding of the robustness of the ideal Pareto Frontier.

\subsection{Curve FitTing of PAREto Frontiers}

Once the Pareto frontier is created it is useful to curve fit the solutions so that points on the Pareto frontier can be quickly evaluated. Unfortunately common curve fitting routines do a poor job of fitting the Pareto frontiers. To fit the Pareto frontiers accurately a Neural Network (NN) is used. A NN provides a fast and accurate way of curve fitting a series of data points. NNs are analog computational systems whose structure is inspired by studies of the brain [48]. This curve fitting technique involves the use of Multilayer Perceptions (MLP) to learn a set of training data and therefore curve fit that data. A MLP consists of a network of units with a series of information passing links or weightings. Each connection of the inputs to the outputs results in a hidden layer between the vectors. The weighting of this mapping between the inputs and the outputs is the learning algorithm used to optimize the network. The number of hidden layers determines the speed and accuracy that the $\mathrm{NN}$ will train. If there are too few hidden nodes then the network will not fit the data; if there are too many hidden nodes then the 
network will take forever to train [49]. A summary of a back-propagation network structure is shown below as Figure 28.

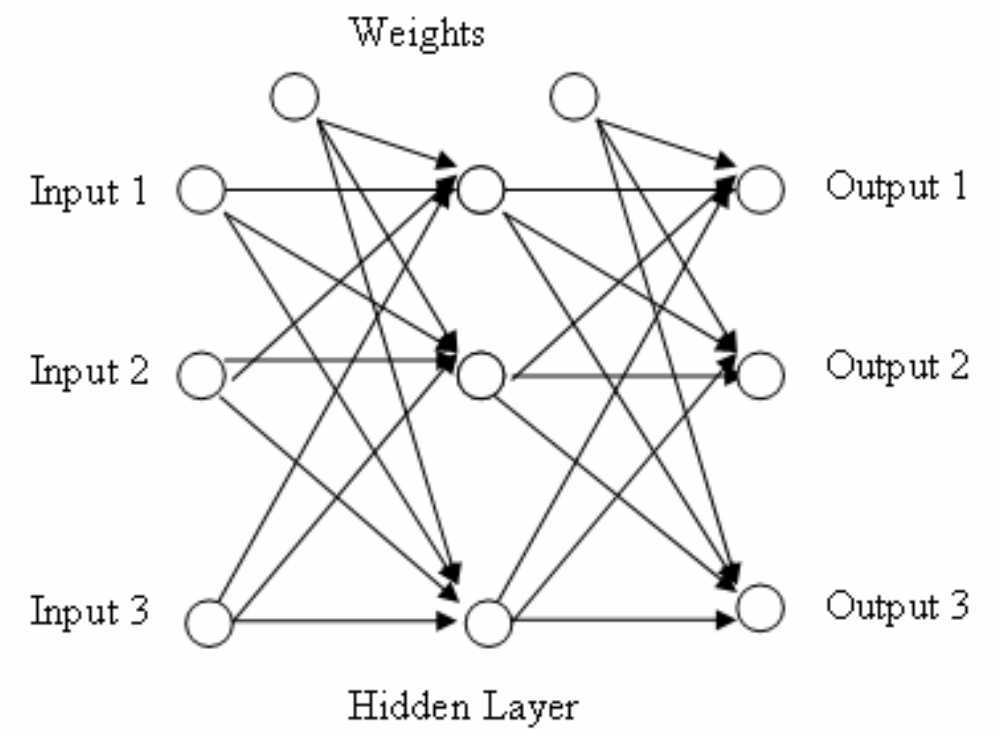

Figure 28: Back-Propagation Neural Network [49].

This figure depicts, a NN with one hidden layer. To curve fit a general Pareto frontier of a 3 hidden node $\mathrm{NN}$ will be used. The resulting equations for the $\mathrm{NN}$ follow as equations 9 and 10.

$$
\begin{gathered}
\text { Response }=\left[B+\left(A_{H 1} * H_{1}+A_{H 2} * H_{2}+A_{H 3} * H_{3}\right)\right] * \sigma_{\mathrm{Rel}}+\mu_{\mathrm{Re} l} \\
H_{i}=\frac{1}{1+e^{-\left(D_{i}+C_{i}^{*}\left(\frac{\text { Cost } \left.\left.-\mu_{\text {Cost }}\right)\right)}{\sigma_{\text {Cost }}}\right.\right.}}
\end{gathered}
$$

Where: A,B,C,D are fitting parameters $\mathrm{H}$ is the hidden node equations 
The resulting curve fits range in $\mathrm{R}^{2}$ values from 0.92 to 0.99 . These resulting curve fits far exceed the curve fits obtained by conventional curve fitting methods.

\subsection{INFORMATION TO DECISION MAKER}

There is a significant amount of research in the operations management community about the need for the decision maker to be provided with not only the optimal solution for the requirements, but also the optimal points for the off nominal requirements. This is especially true in spacecraft design, where life cycle costs can be in the billions of dollars and programs last for decades. The long and costly programs are subject to the whims of the public and funding can either increase or decrease depending on the decision maker leading the project at the time. Because of this instability it is important to have an understanding of the entire design space so that decisions can be made which will be robust to changing project budgets.

Unfortunately although optimal engineering solutions can be found for a given set of requirements, organizational inertia can push these new techniques aside in favor of old less efficient ways. The complications of applying new design techniques to industry are addressed in the literature [50,51,52 ]. As these references demonstrate adoption of a new method, especially a non-transparent method is very difficult regardless of the benefit offered. Because of this non-acceptance, the method proposed in this thesis attempts to address these issues by adapting conventional conceptual design tools into the ROSETTA models through response surface equations. This method also increases the transparency of the process by providing the decision maker with the entire set of solutions in the form of a Pareto frontier. This frontier of optimal solutions allows the decision maker to choose the optimal solution from the best data available and does not 
attempt to hand the decision maker a solution which he/she has not chosen. This allows the decision maker to be invested in the solution to the architecture while avoiding nonoptimal configurations. 


\section{CHAPTER 3}

\section{LUNAR ARCHITECTURE MODE SELECTION}

\section{METHODOLOGY}

Now that the motivation for lunar exploration is set (Chapter 1) and the aerospace design process and the current reliability theories are described (Chapter 2), a new methodology to pull the traditional design discriminators to the front of the design process will be presented. In this chapter, a description of the creation of the ROSETTA models use in this architecture tool will be covered. This description will include the performance models as well as the dynamically changing fault tree analysis and the cost estimation techniques.

The ROSETTA models combined with the conditioned inputs that define the lunar mode to be simulated and a life cycle cost calculation will be combined to provide the tools necessary to create a lunar architecture simulation. The integration of the tools into an integrated framework that can quickly and accurately evaluate the lunar architectures will then be presented. The results of this tool can then be combined into a Pareto frontier to guide the decision maker to producing the highest reliability architecture for a given life cycle cost. 


\subsection{PERFORMANCE ESTIMATION USING ROSETTA MODELS AND RESPONSE}

\section{SURFACE METHODOLOGY}

As noted in Chapter 2, ROSETTA models will be used to estimate the performance, cost, and reliability of the vehicles that constitute the lunar architecture to be simulated. Each of the disciplines in the design structure matrix must be evaluated to complete the performance calculations for the vehicle. As noted in Chapter 2 there are three different levels of fidelity for the ROSETTA models. In this section the creation of the performance section of a level three Lunar Module ROSETTA model will be outline.

\subsubsection{CASE STUDY-LUNAR MOdULE ROSETTA MODEL}

The Lunar Module ROSETTA model is given as an example to demonstrate the procedure to be followed in the creation of a ROSETTA model. The Lunar Module ROSETTA model is a level three model capable of modeling all of the lunar modules implemented in the Apollo mission modes and the ESAS architecture. The design structure matrix for the ROSETTA LM is given below as Figure 29. 


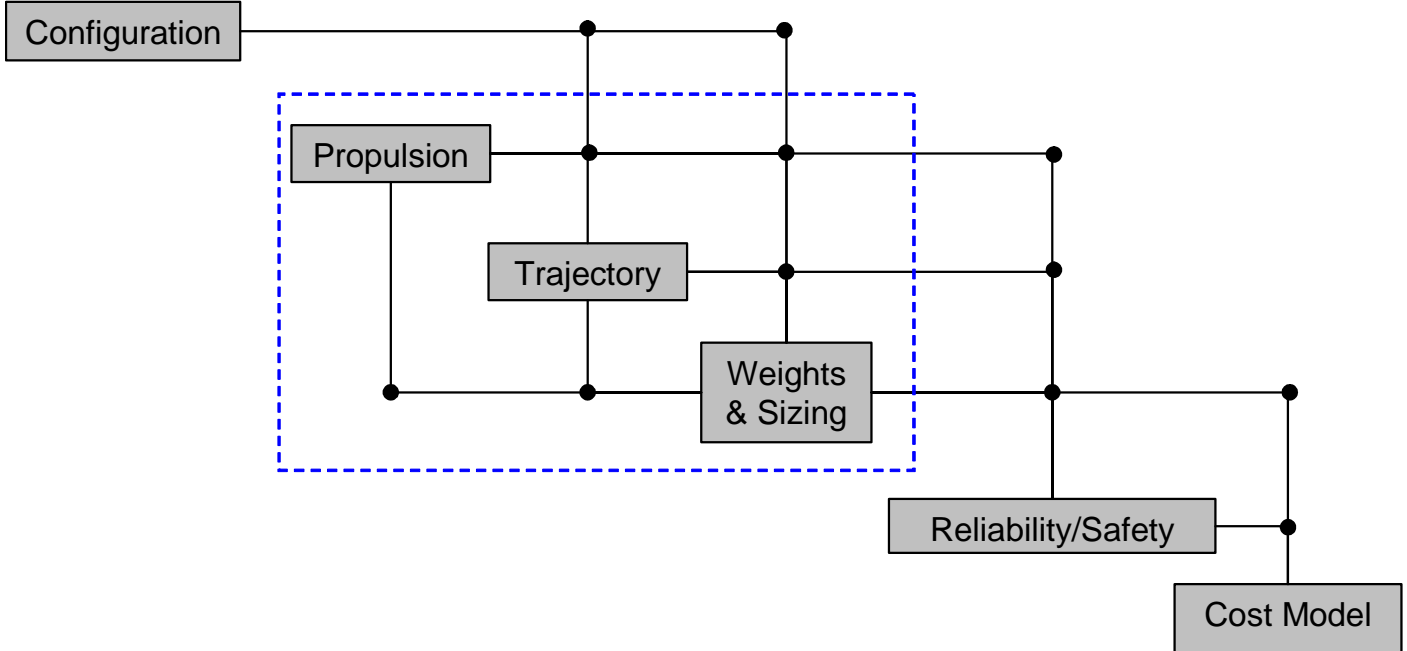

Figure 29: Design Structure Matrix for ROSETTA Lunar Module

As this DSM demonstrates, there are four separate disciplines are integrated to calculate the performance characteristics of the Lunar Module. The reliability and cost models will be addressed in subsequent sections of this chapter. As with most ROSETTA models, there is a strong coupling between the propulsion, trajectory and weights disciplines. This coupling is addressed in the ROSETTA model through iteration in the Excel spreadsheet. This iteration automatically occurs until the inputs to the different CAs are all consistent. This represents a closed vehicle solution.

A lunar module, as defined in this ROSETTA model, must be capable of transporting humans and/or cargo to and from the lunar surface. To be able to simulate all of the Apollo lunar modes as well as the ESAS lunar surface module the ROSETTA model must be robust enough to simulate all of the burns necessary to complete the missions. A summary of the burn profiles for the different lunar landers is included as Table 9 . 
Table 9: Burn Summary of Lunar Landers Simulated in ROSETTA Model

\begin{tabular}{lccccc}
\hline & LOI & Descent & Hover/Landing & Ascent & \# Stages \\
Apollo LOR & & $\mathrm{X}$ & $\mathrm{X}$ & $\mathrm{X}$ & 2 \\
Apollo EOR & & & $\mathrm{X}$ & & 1 \\
Apollo Direct & & & $\mathrm{X}$ & & 1 \\
ESAS & $\mathrm{X}$ & $\mathrm{X}$ & $\mathrm{X}$ & $\mathrm{X}$ & 2 \\
\hline
\end{tabular}

As this table shows, there are multiple configurations that the Lunar Module ROSETTA model must accommodate to simulate all of the lunar mission modes under consideration. In addition to the different trajectory data, the ROSETTA model must be generic enough to simulate manned and unmanned missions (in EOR and Direct the CM acts as the habitat) for any number of crew. The vehicles must also be able to support changing payload weights as the weights of the other vehicles in the mission change. To facilitate these different mission requirements, the ROSETTA model was setup to parametrically scale based upon changing mission requirements. A summary of the ranges of mission requirements that are defined by the ranges of the individual ROSETTA models is given as Table 10.

Table 10: Mission Parameters Ranges for ROSETTA Lunar Module

\begin{tabular}{lcc}
\hline Mission Ranges & Minimum & Maximum \\
Crew Number & 0 & 6 \\
Payload to LS & $500 \mathrm{~kg}$ & $50,000 \mathrm{~kg}$ \\
Payload to LLO & $100 \mathrm{~kg}$ & $50,000 \mathrm{~kg}$ \\
Payload for LOI Burn & $0 \mathrm{~kg}$ & $50,000 \mathrm{~kg}$ \\
Mission time & 3 days & 14 days \\
On Orbit Delay & 0 days & 180 days \\
Number of Stages & 1 & 2 \\
\hline
\end{tabular}


This table gives the mission specific parameters that the ROSETTA model is able to accommodate. There are also over 50 other performance based configuration inputs that the vehicle model uses to simulate a multitude of different lunar landers. These configuration parameters have a large effect on the performance of the vehicle. A summary of some of the configuration parameters is provided as Table 11.

Table 11: Configuration Inputs for ROSETTA Lunar Module

\begin{tabular}{ccc}
\hline Structural Types & Propellant Types & Engine Cycle Types \\
Aluminum & LOX/LH2 & Pressure Fed \\
Aluminum-Lithium & UDMH/N2O4 & Expander \\
Titanium & LOX/CH4 & \\
Graphite Epoxy & & \\
Metal Matrix Composite & & \\
\hline
\end{tabular}

The mission parameters (Table 10), the configuration inputs (Table 11), and the burn profiles (Table 9) are treated as inputs to the design structure matrix and define the configuration for the ROSETTA Lunar Module.

The propulsion discipline involves a Response Surface Equation (RSE) of REDTOP 2 engine performance conceptual design tool [53]. Each propellant combination and cycle type requires a different RSE. These RSEs require the propellant type, cycle type, thrust level, chamber pressure, and $\mathrm{O} / \mathrm{F}$ ratio as inputs. The model then calculates the resulting $\mathrm{I}_{\mathrm{sp}}$ and engine $\mathrm{T} / \mathrm{W}$ to the ROSETTA model [54]. This $\mathrm{I}_{\mathrm{sp}}$ and $\mathrm{T} / \mathrm{W}$ are then passed to the trajectories and weights disciplines.

The trajectory discipline involves a RSE of Program to Optimize Simulated Trajectories (POST 3D) [55]. This program is an industry standard three degrees of 
freedom trajectory simulator that optimizes the weight consumed in a trajectory. The RSE of the POST simulation requires the total vehicle stage weight from the weights analysis and the thrust and Isp from the propulsion analysis [56]. The resulting $\Delta \mathrm{V}$ for the trajectories are then passed to the weights analysis to calculate the total propellant required and the tank sizes.

The weights and sizing discipline uses a series of industry standard Mass Estimating Relationships (MERs) to approximate the vehicle weights [57]. These MERs combined with semi-empirical tank sizing relationships to calculate the size of the tanks necessary to hold the propellant [58]. The structural strengths and densities of the material types are defined from the Humble [59]. This propellant mass is calculated with the trajectory $\Delta \mathrm{V}$ and Isp from rocket equation.

$$
\Delta V_{\text {Traj }}=g_{0} I_{s p} L N\left(\frac{M_{\text {Propellant }}+M_{\text {Inert }}}{M_{\text {Inert }}}\right)
$$

Where: $\mathrm{g}_{0}$ is the gravitational constant

$\mathrm{M}_{\text {propellant }}$ is the propellant mass

$\mathrm{M}_{\text {inert }}$ is the inert mass of the vehicle

The calculated propellant mass is then combined with other MERs to produce the total vehicle mass of the system. This vehicle mass is then iterated with the propulsion (via thrust and $\mathrm{T} / \mathrm{W}$ ratios) and trajectory to close the lunar module vehicle design. A screenshot of the ROSETTA model for the LM is given as Figure 30. 


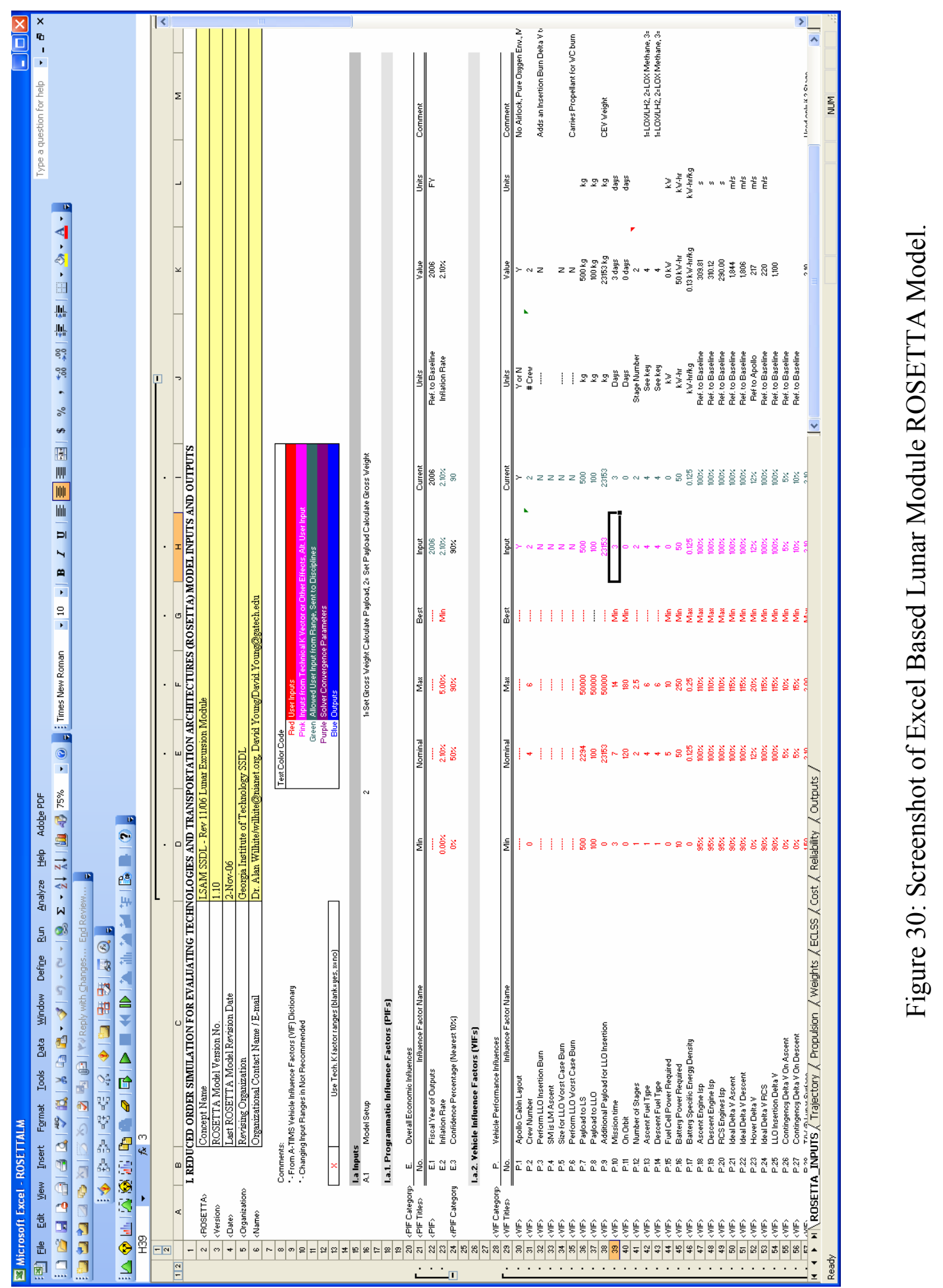


The Excel implementation of the ROSETTA model allows the vehicle's performance to be calculated quickly and is portable to any computer operating Microsoft Office. The resulting mass estimations for the vehicle weight can then be passed to the other ROSETTA models of the lunar architecture.

\subsection{Reliability Calculations Using A Dynamically Changing Fault}

\section{TREE}

To model the reliability and safety disciplines in the ROSETTA model, a new approach was needed. Traditionally, fault trees are used to calculate the reliability of a system based upon each assumed subsystem reliability. This static approach is generally sufficient for calculating the reliability of a system and has been used for all types of systems, including the Space Shuttle. Unfortunately, as described in Chapter 2, the FTA analysis is not sufficient to calculate the reliabilities of changing vehicle configurations since it requires that the number and placement of the subsystems be static to calculate the total reliability of the system. For the methodology of this thesis, it is imperative that the fault trees change dynamically to adjust to the changing vehicle configurations, such as number of engines, propellant types, etc. To accomplish this dynamically changing fault tree, a mathematical representation of the fault tree was created in Excel and was allowed to change based upon the input vehicle configurations. These dynamically changing fault trees will be implemented in every ROSETTA model to accurately calculate the resulting vehicle reliability based upon the changing configurations. The differences between a lunar lander with engine out and a generic lunar lander without engine out are shown as Figure 40 and Figure 41. 
These dynamically changing fault trees must be able to not only calculate the loss of mission reliability of a vehicle but also the loss of crew safety. These calculations differ by the severity of the failure and the ability of the crew to survive the failure type. For most instances, the difference between the types of failures will depend on the vehicle and the subsystem which failed. For most models, the difference between LOM and LOC will be established via a percentage of subsystem failures that result in a LOC event. Even if a LOC event occurs, it does not necessarily result in a loss of crew. Some vehicles have emergency abort systems that will save the crew in the event of a LOC event occurs. The success of these systems is still subject to the reliability of the crew abort system. For vehicles with such an abort system, the LOC number is inflated by the reliability of that abort system via a parallel reliability calculation.

$$
L O C=L O C_{\text {event }} *\left(1-L O C_{\text {event }}\right) * R_{C E S}
$$

Where: LOC is the loss of crew probability of the system

$\mathrm{LOC}_{\text {event }}$ is the probability of a loss of crew event with the vehicle

$\mathrm{R}_{\mathrm{CES}}$ is the reliability of the crew escape system

A summary of the individual subsystem reliability assumptions that are included in the reliability and safety analysis follow.

\subsubsection{GENERAL SUBSYSTEM RELIABILITY MODELING METHODS}

The dynamic fault tree analysis integrated into this methodology requires that the individual subsystem reliabilities be defined and input into the base of the fault tree. These individual subsystem reliabilities are generally considered to be inputs into the 
vehicle fault tree. In the case of a propulsion subsystem, the subsystem reliabilities are highly dependant on the configuration of the vehicle. The individual element reliabilities can be found in literature, but models have to be developed to calculate the total subsystem reliability that can be included in the dynamic fault tree analysis.

The propulsion subsystem reliability is a combination of different reliability models combined to account for the possible failure modes of the each subsystem. These reliability models include:

- Common Cause Failure Model

- Catastrophic Engine Failure Model

Each of these models is combined into one branch of the dynamically changing fault tree analysis to provide the overall propulsion system reliability.

\section{Common Cause Failure Model}

Common Cause Failure (CCF) is a reliability technique that attempts to model the failures that are inherent due to flaws in all redundant systems in an element. A standard definition of common cause failure is, "A subset of dependent events in which two or more component fault states exist at the same time, or in a short interval, and are direct result of a shared cause." [60]. These CCFs usually occur in systems where multiple, usually redundant, components all share the same flaw. This flaw can be a manufacturing, software, or other problem that is common to all redundant systems in an element. An example of a CCF is a flaw in the materials properties found in many propellant tanks, which cause all tanks in a system to fail when stressed. In this case a redundant system, such as multiple tanks of a fuel cell in a power system, can fail 
simultaneously due to a materials flaw in the construction of many propellant tanks. Common cause failures are not uncommon in today's spacecraft. The space shuttle program recently had a suspected instance in a common cause failure when the electronic fuel cutoff switches in the external tank delayed the July launch of the Space Shuttle Discovery [61]. There are four hydrogen fuel sensors in the space shuttle external tank. It was suspected when one of the sensors failed that a manufacturing flaw could have possibly caused all of the sensors to fail. This fear of a common cause failure caused the Discovery launch to be delayed nearly a month.

There are many proposed methods to model CCF. These methods include both explicit and implicit methods of modeling a CCF [62]. An explicit method treats the common cause as a separate event in the fault tree analysis. An implicit method treats the common cause failure as an algebraic unreliability expression, which is then included in the reliability calculation input into the fault tree analysis. In the methodology described in this thesis, an explicit method is chosen for extensibility because it is generally difficult to derive an algebraic expression of system unreliability [63].

There are many different explicit methods that can be chosen to model common cause failures in a dynamic fault tree analysis. In this methodology a single parameter or $\beta$ model will be used [64]. A single parameter model is a model that uses a single $\beta$ parameter in parallel with the redundant components in a subsystem. This $\beta$ parameter is defined as a combination of independent failure rates and common cause failure rates as given by equation 13 . 


$$
\beta=\frac{\lambda_{c}}{\lambda_{c}+\lambda_{I}}
$$

Where: $\lambda_{\mathrm{c}}=$ failure rate due to common cause failures $\lambda_{\mathrm{I}}=$ failure rate due to independent failures

This single parameter model is explicit and is therefore treated as an independent event in series with the component failure rate in the fault tree analysis. An example of a common cause in a simple two component redundant system is shown as Figure 31.

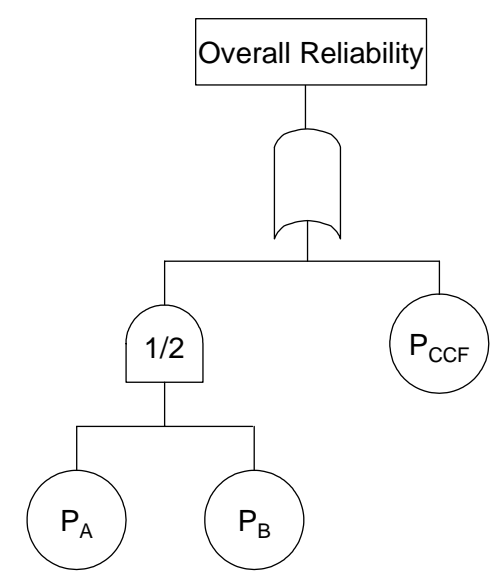

Figure 31: Common Cause Failure Implementation into a Single Redundant System.

As this fault tree shows the common cause failure is treated as a separate event, which is added in series with the redundant system. For most cases the single parameter failure rate can be treated as a set value. For this research a failure rate value of 0.10 was chosen based upon the work by Mosleh, et al. [60]. In this methodology, redundant systems will all be considered to have a common cause failure term. Redundancy will be treated as a 
$\mathrm{k}$ out of $\mathrm{n}$ system (for multiple redundancies) with a single parameter common cause failure added in series to this system.

\section{Catastrophic Engine Failure Rate}

Common cause failures are not the only specific breakdown of the subsystem system reliability used in this methodology. Failure rates are broken down in this research according to the four types of failures defined in Huang et al [66]. This breakdown divides failure rates into four categories:

- Catastrophic Failures

- Non-Catastrophic Failures

- Preventable Catastrophic Failures

- Unnecessary Shutdown Failures

The definition of these failures as well as their implementation within this research follows.

A catastrophic failure is defined as an uncontained failure the immediately results in a loss of vehicle. This, in turn, will result in a loss of crew unless a crew abort system is in place. Historically, catastrophic failure rates for engines range between $20 \%$ and $40 \%$ [66]. This catastrophic failure rate was generally derived for propulsion subsystems but is generalized for all subsystems.

Non-catastrophic failure rates are considered failures that are contained by the failing element. An example of this is a failure that causes the system to shutdown, but the vehicle can still operate with a redundant system. The aforementioned shuttle main engine cutoff sensors are an example of a non-catastrophic failure. If the sensor fails 
"wet," or the engine shuts down when fuel is still present, the failure is contained to this subsystem. Since the ECO sensors have redundancy, this failure would not result in a loss of mission.

The third type of failure is the preventable catastrophic failure. This type of failure would result in a catastrophic failure if the safety systems did not shut down the operating equipment. An example of this would be an engine health management system shutting down an engine when redlines are exceeded. If the health management system was not present, a catastrophic failure would have occurred; but it was prevented by the safety system.

The fourth and final type of failure is unnecessary shutdown failures. These failures are a result of the safety systems sensor failure, where the operating element is shut down when no real failure has occurred. This can be found in almost any avionics or health management system where redlines are too restrictive.

Each of these types of failures is represented as basic events in the fault tree analysis. These failure rates can then be combined with the common cause failure rate to produce an entire subsystem failure rate. A representation of what a propulsion system fault tree would look like with the CCF and catastrophic engine failure rates are included is shown as Figure 32. 


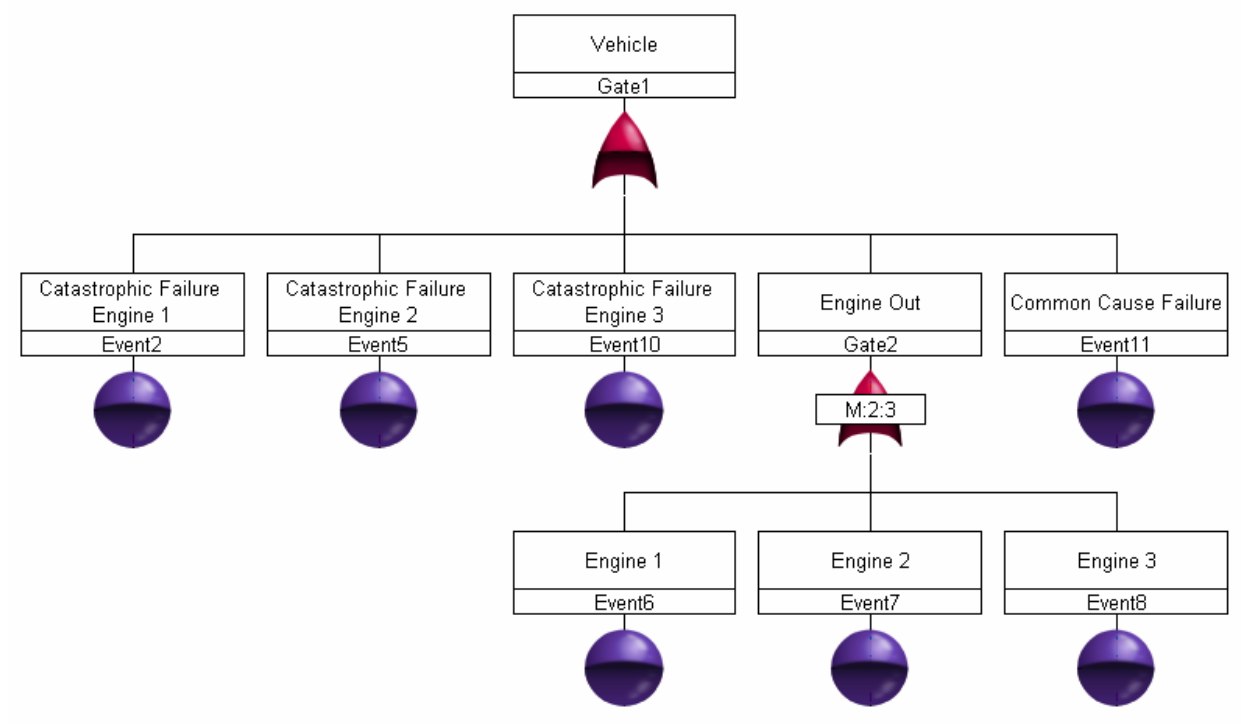

Figure 32: Fault Tree Representation of the Propulsion System for a Generic Launch Vehicle

This figure shows a three engine propulsion system with engine out vehicle configuration represented as fault tree. As this figure shows the catastrophic failure fraction rate for each engine must be treated as an independent event. A catastrophic failure of any of the engines causes a failure of the entire vehicle. Common cause failure is also treated much like a catastrophic failure rate in that it is an independent event which is in series with the actual elements of the vehicle. The engine out system is treated as a $\mathrm{k}$ out of $\mathrm{n}$ system. It should be noted that although the $\mathrm{k}$ out of $\mathrm{n}$ system increases the reliability, the addition of an extra engine catastrophic rate decreases the reliability over an ideal $k$ out of $n$ system.

To complete a dynamic fault tree analysis this fault tree must be mathematically described in the ROSETTA model. To mathematically model this reliability, a catastrophic engine failure fraction was added to a $\mathrm{k}$ out of $\mathrm{n}$ system. The mathematical model derived from Huang et al. is given [66]: 


$$
\begin{aligned}
& R_{P S}=\left[1-\left(C_{F}\left(1-R_{T}\right)+P_{F}\left(1-R_{T}\right)+U_{F}\left(1-R_{T}\right)+N C F_{F}\left(1-R_{T}\right)\right)\right]^{N} \\
& R_{P S_{(E O)}}=\left(1-C_{F}\left(1-R_{T}\right)\right)^{N}\left[\left(1-\left(1-C_{F}\right)\left(1-R_{T}\right)\right)^{N}+\right. \\
& \left.N\left(1-\left(1-C_{F}\right)\left(1-R_{T}\right)\right)^{N-1}\left(1-C_{F}\right)\left(1-R_{T}\right)\right]
\end{aligned}
$$

Where: $R_{P S}$ is the reliability of the propulsion system

$\mathrm{R}_{\mathrm{PS}(\mathrm{EO})}$ is the reliability of the propulsion system with engine out

$\mathrm{C}_{\mathrm{F}}$ is the catastrophic failure rate

$\mathrm{R}_{\mathrm{T}}$ is the single engine reliability

$\mathrm{P}_{\mathrm{F}}$ is the preventable failure fraction

$\mathrm{U}_{\mathrm{F}}$ is the unnecessary shutdown failure fraction

$\mathrm{NCF}_{\mathrm{F}}$ is the non-catastrophic failure fraction

$\mathrm{N}$ is the number of engines

This model for loss of mission reliability can be extended to calculate the loss of crew reliability. The loss of crew number includes a factor to account for the fact that the crew may survive a non-catastrophic failure. That factor is included as a vehicle stage specific percentage of the trajectory where the crew can successfully survive a non-catastrophic failure. The inclusion of this trajectory percentage for loss of crew follows.

$$
R_{P S}=\left[1-\left(C_{F}\left(1-R_{T}\right)+\left[P_{F}\left(1-R_{T}\right)+U_{F}\left(1-R_{T}\right)+N C F_{F}\left(1-R_{T}\right)\right]^{*}(1-\% T)\right)\right]^{N}
$$




$$
\begin{aligned}
& R_{P S_{(E O)}}=\left(1-C_{F}\left(1-R_{T}\right)\right)^{N}\left[\left(1-\left(1-C_{F}\right)\left(1-R_{T}\right)(1-\% T)\right)^{N}+\right. \\
& \left.N\left(1-\left(1-C_{F}\right)\left(1-R_{T}\right)(1-\% T)\right)^{N-1}\left(1-C_{F}\right)\left(1-R_{T}\right)(1-\% T)\right]
\end{aligned}
$$

Where: $\% \mathrm{~T}$ is the percentage of the trajectory where the crew can survive a non-catastrophic failure without a crew escape system $\mathrm{R}_{\mathrm{PS}}$ is the reliability of the propulsion system

$\mathrm{R}_{\mathrm{PS}(\mathrm{EO})}$ is the reliability of the propulsion system with engine out

These loss of mission and loss of crew systems representations can be combined with the common cause failure rate to calculate a total loss of mission and loss of crew expression. A summary of the analytical equations for the loss of mission and loss of crew reliabilities follow.

Loss of Mission:

$$
\begin{aligned}
& R_{P S}=\left[1-\left(C_{F}\left(1-R_{T}\right)+C C F\left(1-R_{T}\right)+P_{F}\left(1-R_{T}\right)+U_{F}\left(1-R_{T}\right)+N C F_{F}\left(1-R_{T}\right)\right)\right]^{N} \\
& R_{P S_{(E O)}}=\left(1-C_{F}\left(1-R_{T}\right)\right)^{N}\left[\left(1-\left(1-C_{F}-\beta\right)\left(1-R_{T}\right)\right)^{N}+\right. \\
& \left.N\left(1-\left(1-C_{F}-\beta\right)\left(1-R_{T}\right)\right)^{N-1}\left(1-C_{F}-\beta\right)\left(1-R_{T}\right)\right]\left(1-\beta^{*}\left(1-R_{T}\right)\right)
\end{aligned}
$$

\section{Loss of Crew:}

$$
\begin{aligned}
& R_{P S}=\left[1-\left(C_{F}\left(1-R_{T}\right)+C C F\left(1-R_{T}\right)+\left[P_{F}\left(1-R_{T}\right)+\right.\right.\right. \\
& \left.\left.\left.U_{F}\left(1-R_{T}\right)+N C F_{F}\left(1-R_{T}\right)\right]^{*}(1-\% T)\right)\right]^{N} \\
& R_{P S_{(E O)}}=\left(1-C_{F}\left(1-R_{T}\right)\right)^{N}\left[\left(1-\left(1-C_{F}-\beta\right)\left(1-R_{T}\right)(1-\% T)\right)^{N}+\right. \\
& \left.N\left(1-\left(1-C_{F}-\beta\right)\left(1-R_{T}\right)(1-\% T)\right)^{N-1}\left(1-C_{F}-\beta\right)\left(1-R_{T}\right)(1-\% T)\right]\left(1-\beta *\left(1-R_{T}\right)\right)
\end{aligned}
$$


Where: $\beta$ is the common cause failure percentage

These equations are implemented in the ROSETTA models to address the subsystem reliabilities that are compiled in the development of the dynamically changing fault tree analysis.

\subsubsection{PROPULSION SYSTEM RELIABILITY ModELING}

Propulsion systems have multiple drivers that can lead to an engine failure. This research breaks down the propulsion system reliabilities into different categories. The propulsion system can be affected by:

- Engine Cycle Type

- Propellant Type

- Reusability

- De-rating Engines

- Thrust Level

The engine cycle type can affect the engine system reliability because the cycle type is a good indication of the complexity of the engine. In this research, there will be two types of inspace engine cycle types considered and two types of launch vehicle engine cycle types considered. For inspace engines, both pressure fed and expander cycle engines are considered. These two types of engines are chosen to model all of the engines considered in the Apollo and ESAS mission mode architectures. A pressure fed engine is a simple system that stores the propellants at high pressures in the propellant tanks. This high 
pressure provides the propellant transfer from the tanks to the combustion chamber. This simple method results in a highly reliable engine but also results in higher weight, higher cost tanks. An expander cycle is an engine cycle that uses the heating of the fuel in the nozzle to power the turbines of the pumps. These pumps increase the complexity of the engine, but lower the tank weights since the tanks are allowed to operate at lower pressures. A summary of the expander cycle for inspace engines is given below as Figure 33.

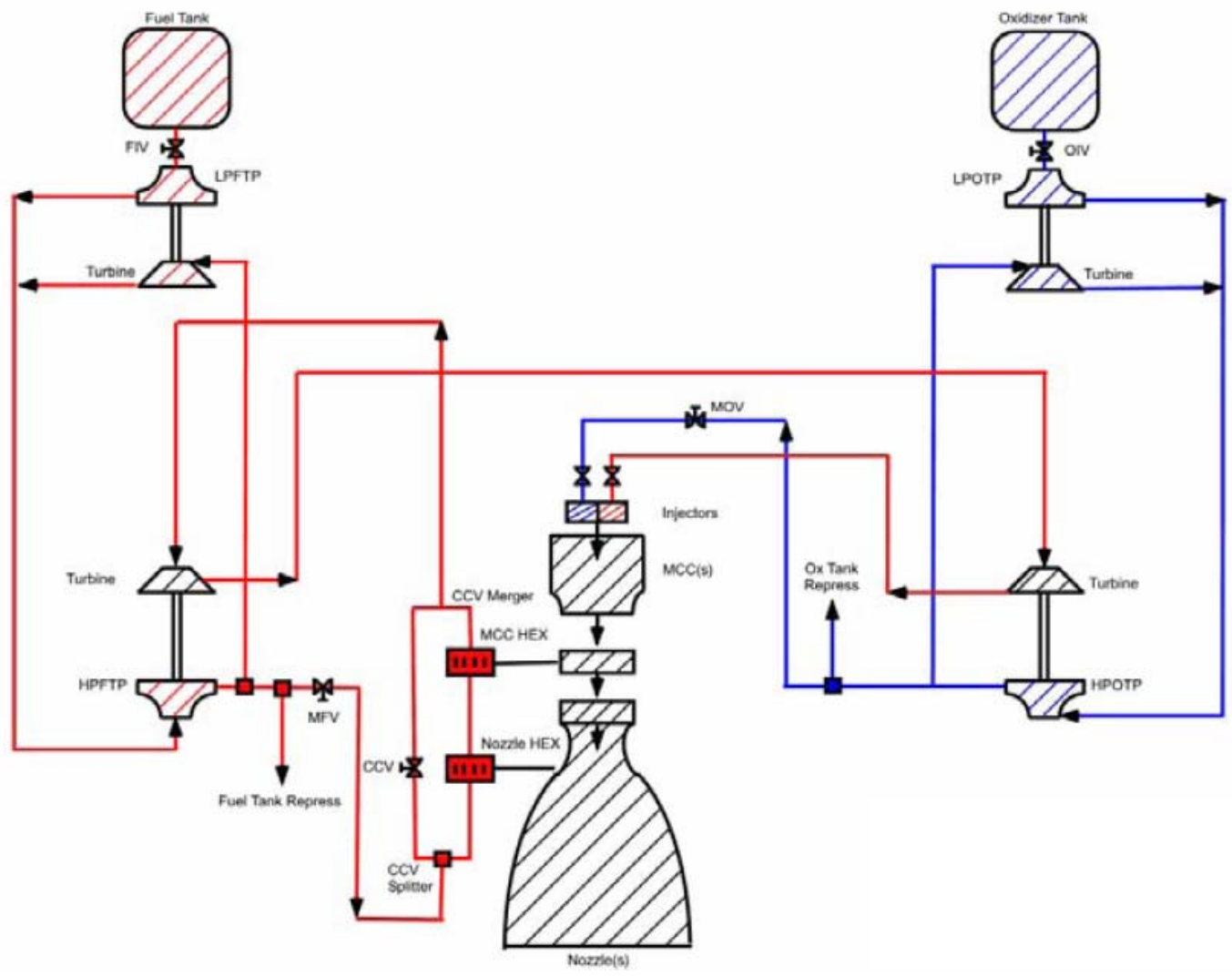

Figure 33: Expander Cycle Engine Schematic [65]. 
There are two types of launch vehicle engine cycle types considered. The first cycle type is the gas generator cycle. This cycle is widely used in launch vehicles and has been used on the Saturn and Delta family of launch vehicles. The gas generator cycle utilizes a smaller combustion chamber or gas generator that provides the pressures to run the turbine systems. These turbines then power the pumps that push the propellant to the combustion chamber and the gas generator. A schematic of the gas generator cycle is included as Figure 34.

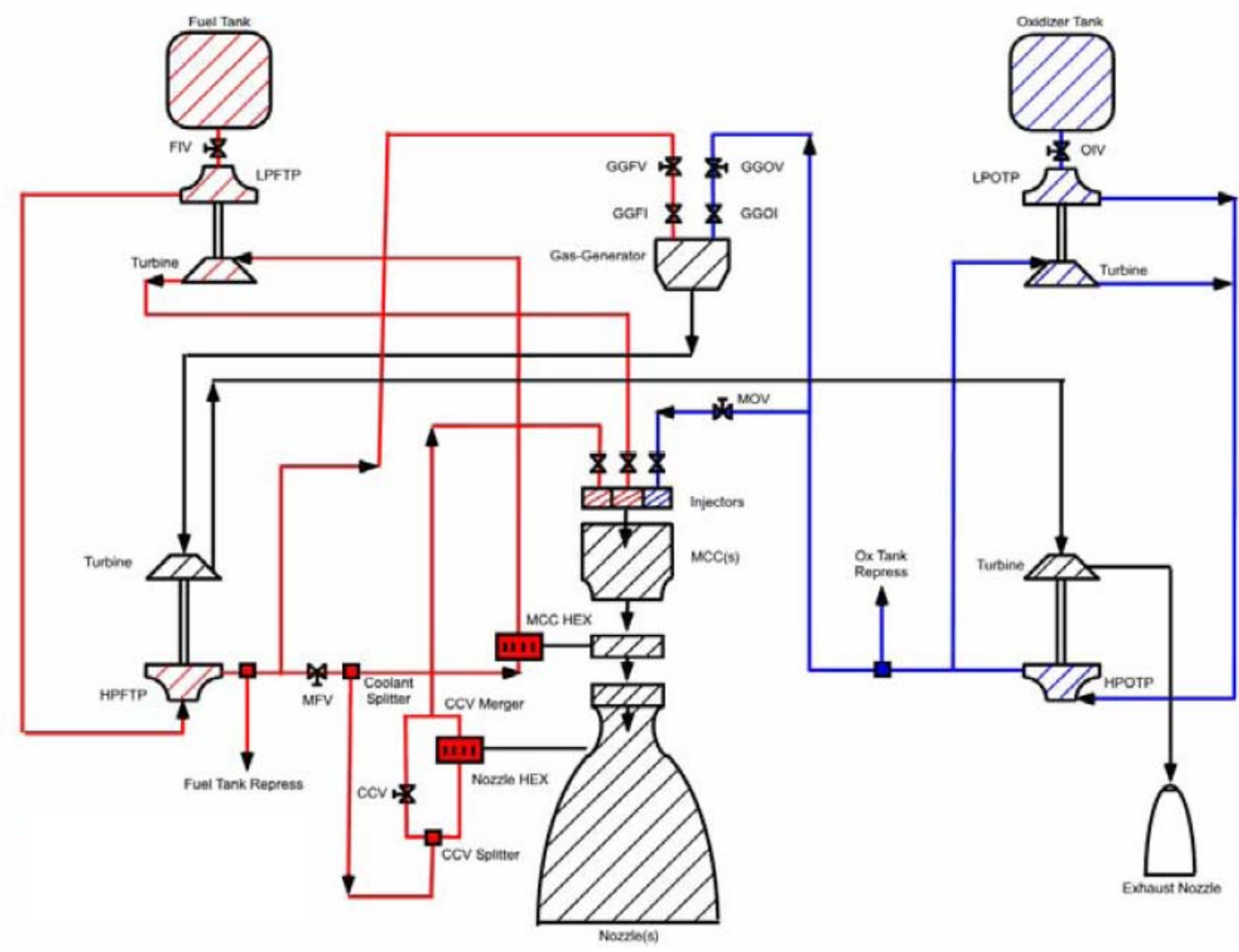

Figure 34: Schematic of Gas Generator Engine Cycle [65] 
The final type of engine cycle considered is the staged combustion cycle. This cycle involves the presence of a preburner, which begins the combustion process by burning a small amount of propellant. This combustion process drives the pumps and preheats the propellants as they enter the combustion chamber. A staged combustion cycle type is the most complicated of the cycle types; but, since all of the propellant travels through the main nozzle, it provides the highest efficiency. A schematic of a fuelrich staged combustion cycle is given as Figure 35.

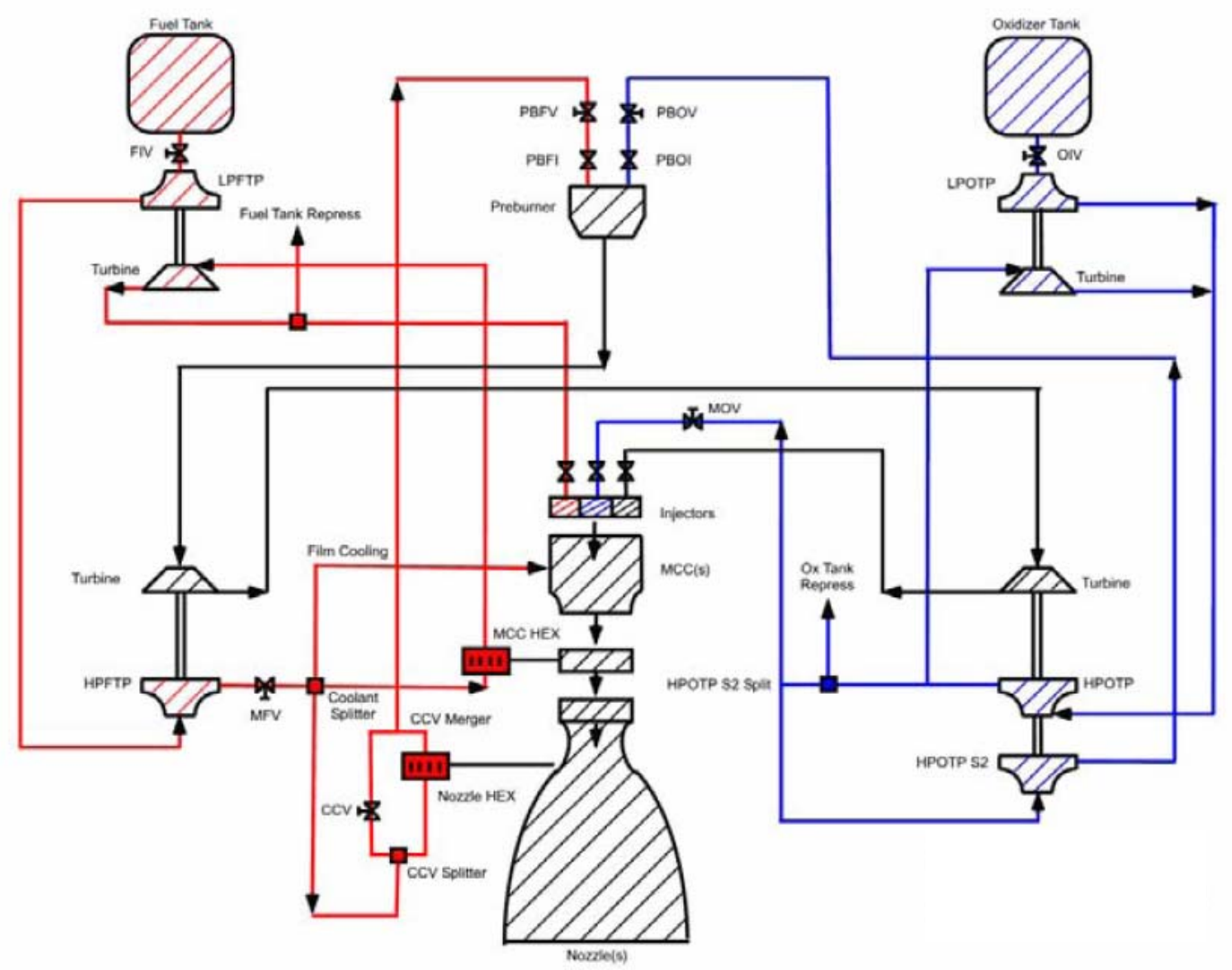

Figure 35: Schematic of Staged Combustion Engine Cycle [65] 
As these figures show, the complexity, and therefore reliability, of each of propulsion subsystems varies depending on the propellant and engine cycle type. These differences are accounted for by using table look-ups of propellant and engine cycle types as baseline reliabilities for the engines used in the ROSETTA models.

Reusability is another factor that can affect the reliability of a propulsion system. Reusability can increase the single flight reliability of an engine since reusable engines tend to be over designed for a single mission. This over design results in higher design margins and therefore increased reliabilities. Reusability also allows the engines to be studied post-flight and therefore possible problems can be addressed early and redesigns are possible before failures occur. In the lunar architectures addressed in this research, there are no reusable elements and therefore reusability is not directly addressed. Reusable engines are used in certain architectures addressed in this research and therefore the higher single mission reliability is accounted for in the reliability baselines.

De-rating of engines is another method that can be used to increase the reliability of a system. De-rating of a system involves operating an engine at a lower power level that the maximum design power level of the engine. Historical evidence indicates that about $90 \%$ of failure modes and causes are power level related [66]. Models exist that attempt to quantify the reliability change as a function of the power level of the engine. This model is defined as equation 22 [67]:

$$
P_{\text {Failure }}(R u b b)=P_{\text {Failure }}(\text { Nom })\left[\operatorname{TIFF}+(1-T I F F) E X P\left(\frac{\Delta P L}{R F}\right)\right]
$$

Where: TIFF is Throttle Insensitivity Failure Fraction 
$\mathrm{PL}$ is the operational power level

$\mathrm{RF}$ is reliability based factor dependant on fuel type

This equation defines the de-rated reliability of an engine as a function of the $100 \%$ throttle reliability and multiplicative factors that are a function of the propellant type. A graph of the reliability as a function of throttle level for the Space Shuttle Main Engine (SSME) is calculated from equation 10 and the TIFF factor defined in the ESAS final report [13]. The plot of this reliability for the SSME is given as Figure 36.

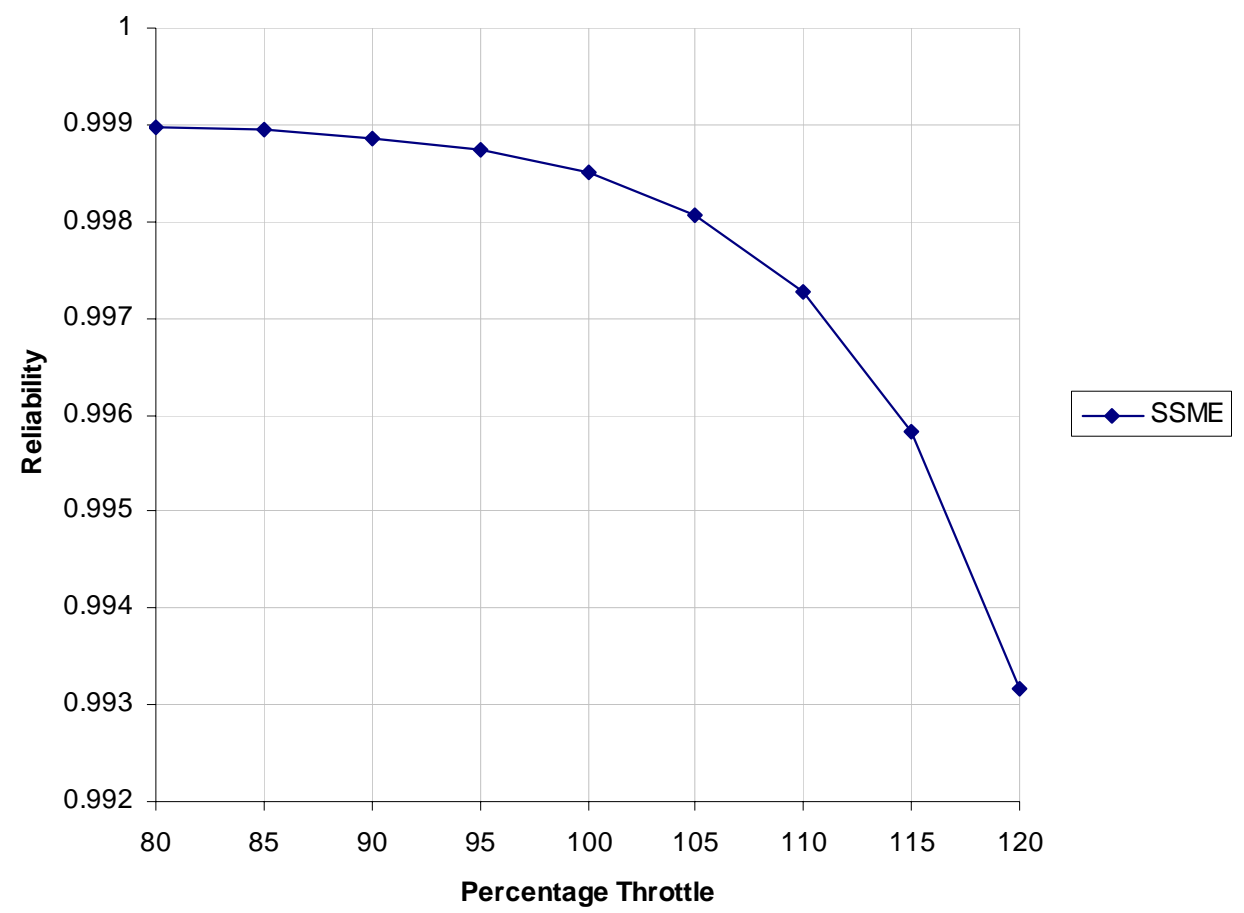

Figure 36: De-rating Engine Reliability for the Space Shuttle Main Engine.

As this figure indicates, the reliability of an engine falls off dramatically as the throttle percentage increases above $100 \%$. The de-rating of the engine only increases the 
reliability slightly; and therefore, all engines will be assumed to run at $100 \%$ throttle for this research.

A final driver considered for the propulsion analysis is the reliability of changing the thrust level of an engine when compared to the existing baseline engine. The reliability of every vehicle propulsion system changes as the vehicle scales. This occurs because the engine reliability is a function of the size (thrust) of an engine. In conceptual vehicle design, it is common to "rubberize" engines, or increase the engine thrust level as the vehicle grows. The relationship between engine size and engine failure rate is established as a power function when compared to a baseline engine [67]. This relationship is given below:

$$
P_{\text {Failure }}(\text { Rubb })=P_{\text {Failure }}(B L)\left(\frac{\text { RatedThrust }}{\text { RatedThrust }_{B L}}\right)^{0.1017}
$$

Where: $\mathrm{P}_{\text {Failure }}(\mathrm{Rubb})$ is the probability of failure of the rubberized engine $\mathrm{P}_{\text {Failure }}(\mathrm{BL})$ is the probability of failure of the baseline engine RatedThrust is the new thrust level for the rubberized engine RatedThrust $_{\mathrm{BL}}$ is the thrust level of the baseline engine

In this reliability model the baseline engine is the propellant type and engine cycle type of the rubberized engine being evaluated. This equation was evaluated for a staged combustion LOX/RP1 engine (RD-180) and a staged combustion LOX/LH2 engine (SSME). The resulting reliability as a function of rubberized engine thrust is given as Figure 37. 


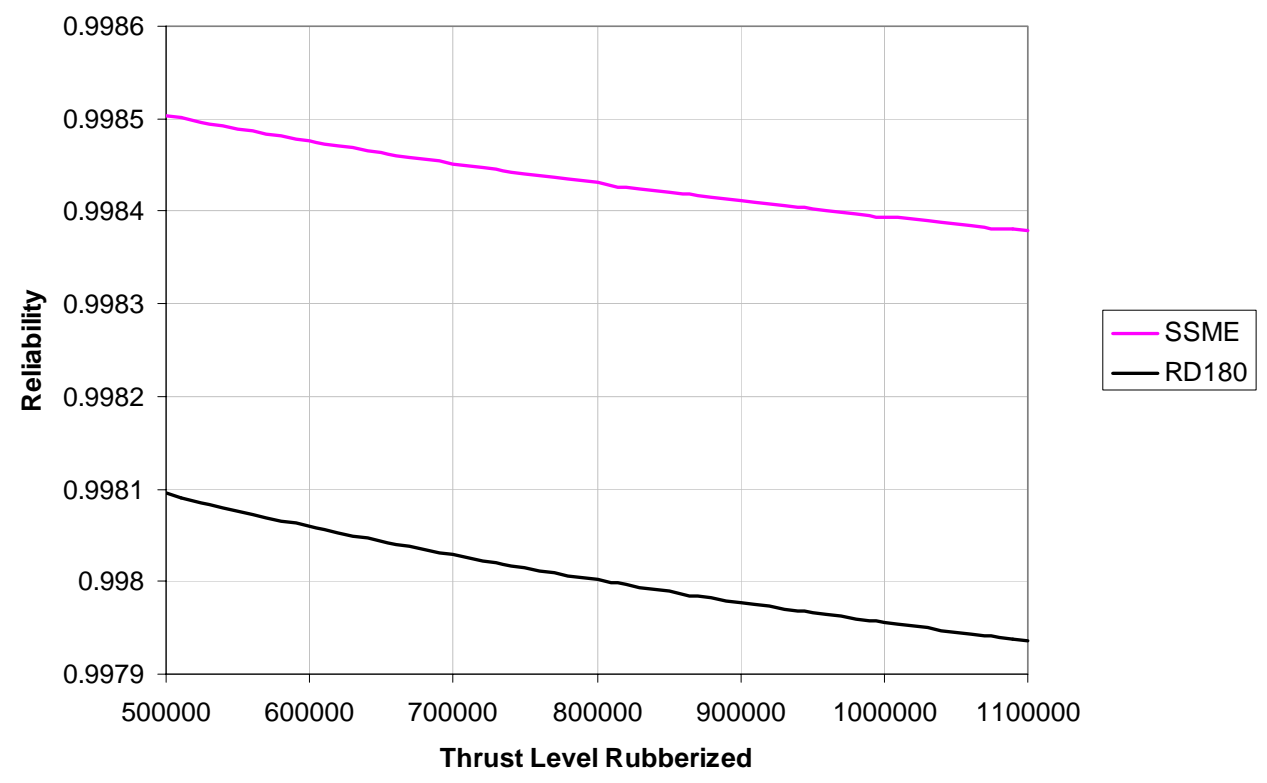

Figure 37: Rubberized Engine Reliability for the SSME and RD-180 based Engines

As this figure depicts, the total engine reliability is a weak function of the thrust of the engine. The RD-180 reliability is significantly lower than the SSME baseline reliability. This difference in reliability is due to the amount of inspection and evaluation that has gone into the reusable SSME as apposed to the expendable RD-180. This model was incorporated into the ESAS final report and will be used in the propulsion reliability calculations for the dynamically changing fault trees of the ROSETTA model.

At the beginning of this research, engine testing was also considered a major driver for the total reliability of an engine. An evaluation of existing engine data indicates that propulsion system configuration (propellant type and cycle type) is independent of the increase in reliability due to testing. This difference between the inherent reliability and the demonstrated reliability is therefore constant for all engine models. A summary of the testing model from Transcost is given below [38]: 


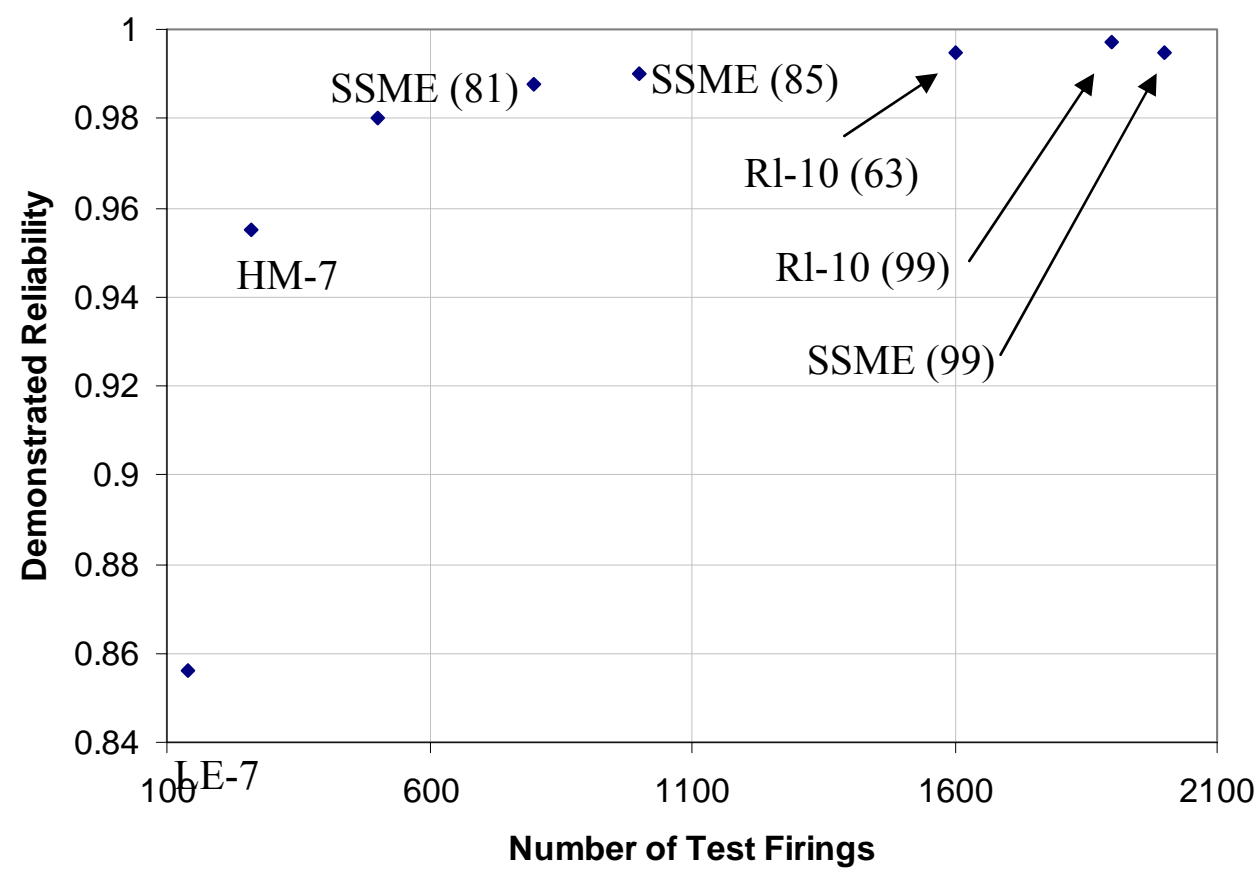

Figure 38: Engine Demonstrated Reliability as a Function of Testing

As figure shows, there is a relationship between the number of engine tests and the total demonstrated reliability of the engine. This relationship is independent of the number of test firings and will therefore not affect the lunar architecture selections that are made with this methodology.

The fact that there is a relationship between testing and reliability should not be completely ignored. Even though the lunar architecture selection tool will not optimize based upon engine testing, the ability to model the increased cost and reliability of the system due to testing should be incorporated into the ROSETTA models. To accomplish the increased cost with testing, a $\mathrm{K}$ factor was applied to the costing model as a function of the required number of engine tests to achieve the desired inputted engine reliability. This cost multiplier is described in Transcost and is presented below [38]. 


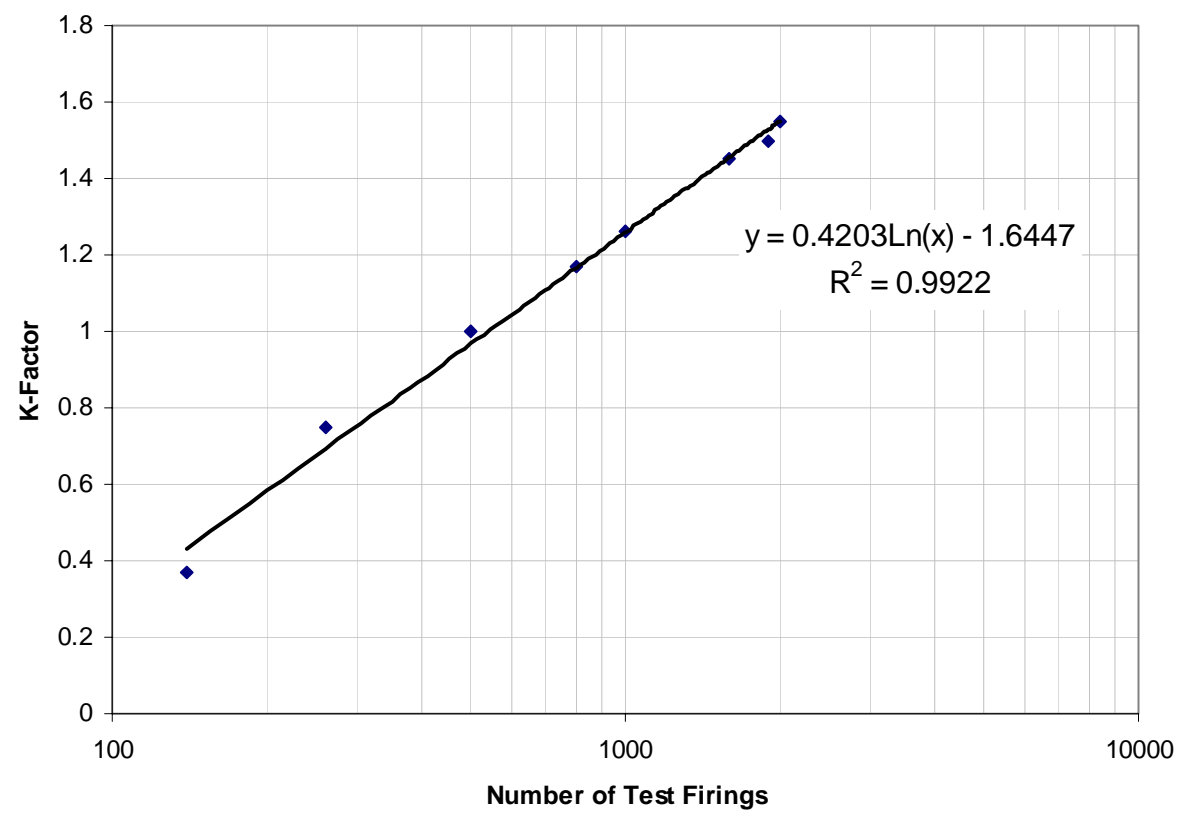

Figure 39: Costing K Factor for Engine Testing

As this figure shows the K-factor for the cost of engine testing can either increase or decrease the total design, development, testing, and evaluation costs by up to $50 \%$. The costing model included in the ROSETTA models will be discussed in detail in Section 3.3 .

As the vehicle propellant type changes the total reliability and cost of that vehicle changes. For example as the propellant of a Lunar module changes from a UDMH/N2O4 pressure fed engine to an LOX/LH2 expander cycle engine the performance, cost, and reliability of the vehicle changes. A comparison between each propellant type for a lunar lander using Apollo LOR requirements and pressure fed engines is given below as Table 12, while the expander cycle propellant comparisons are given as Table 13. 
Table 12: Comparison of Different Propellant Types for a Pressure Fed Apollo LM

\begin{tabular}{lccc}
\hline & UDMH/N2O4 & $L O X / C H 4$ & $L O X / L H 2$ \\
Dry Mass & $4,216 \mathrm{~kg}$ & $5,433 \mathrm{~kg}$ & $7,863 \mathrm{~kg}$ \\
Gross Mass & $16,183 \mathrm{~kg}$ & $18,114 \mathrm{~kg}$ & $19,875 \mathrm{~kg}$ \\
LOM & 0.8918 & 0.8297 & 0.7942 \\
LOV & 0.8941 & 0.8318 & 0.7962 \\
\hline
\end{tabular}

Table 13: Comparison of Different Propellant Types for an Expander Cycle Apollo LM

\begin{tabular}{lccc}
\hline & UDMH/N2O4 & LOX/CH4 & LOX/LH2 \\
Dry Mass & $3,202 \mathrm{~kg}$ & $3,423 \mathrm{~kg}$ & $3,738 \mathrm{~kg}$ \\
Gross Mass & $12,474 \mathrm{~kg}$ & $11,867 \mathrm{~kg}$ & $10,436 \mathrm{~kg}$ \\
LOM & 0.8818 & 0.8807 & 0.8652 \\
LOV & 0.8841 & 0.883 & 0.8674 \\
\hline
\end{tabular}

As these tables show, the dynamically changing fault trees account for the different engine cycle types and propellant types chosen in the ROSETTA models. In comparing the pressure fed engines the engine reliabilities are higher for the hypergolic propellants, but are lower for the cryogenic LOX/CH4 and LOX/LH2 propellants. This is a result of the increased tank sizes necessary for the pressure fed engines and the low densities of the cryogenic propellants. As the engine cycle type is changed to expander cycle the tank weights drop significantly and the engine reliabilities play a much larger role in the vehicle reliability. Therefore the hypergolic propellants have the highest reliability followed by the lower density higher energy cryogenic propellants. 


\subsubsection{STRUCTURAL SYSTEM RELIABILITY MODELING}

As with the propulsion subsystem the structural subsystem must also change with the changing vehicle configurations. Generally, five different structural materials will be considered in this methodology. These five structural materials are:

- Aluminum

- Aluminum-Lithium

- Titanium

- Graphite Epoxy (or Graphite Epoxy Wrapped Aluminums for propellant tanks)

- Metal Matrix Composites

Of these five materials, aluminum and aluminum-lithium are widely used in spacecraft structures. Titanium and graphite epoxy are widely used in aerospace systems, and metal matrix composites is new material that is being tested to be used in high strength light weight applications [68]. It is considered in the ROSETTA models that titanium, aluminum, and aluminum-lithium have high reliabilities due to the common use of these materials. Graphite epoxy has a slightly lower reliability due to the fact that it is relatively new in manned spacecraft applications. Metal matrix composites have the lowest reliability of any of the material due to the fact that they have never been used in manned spacecraft designs.

To model the changing reliabilities due to structural changes a table lookup is used to change the baseline reliability of the subsystem. This baseline reliability is then compiled with the catastrophic and common cause failure rates to get the reliability of the tank subsystems. The primary and secondary structural reliabilities are calculated much 
the same way as the tank calculations. A summary of these reliabilities is given in Appendix B.

\subsubsection{Other SubSystems Modeling TECHNIQUE}

Each of the subsequent subsystems in the vehicle ROSETTA models is modeled in much the same way as the structural reliabilities. The baseline reliability numbers are derived from the vehicle specific Apollo subsystem reliabilities [69]. These reliabilities are then adjusted for the ESAS ROSETTA models to adjust the Apollo reliabilities to account for increased reliability gained due to increased use and verification since the Apollo program. This factor is vehicle specific and will be addressed in Chapter 4. A summary of the subsystem reliabilities is given in Appendix B.

\subsubsection{CASE Study-Lunar Module ROSETTA Model RELIABILITY Model}

Once the individual subsystem reliabilities are mathematically modeled the subsystems are compiled into one vehicle model. This model is then incorporated into the ROSETTA mode and designed to change with the changing vehicle inputs. As these vehicle inputs change the fault tree must also change. The problem with static fault trees is demonstrated in the following figures Figure 40 and Figure 41. 


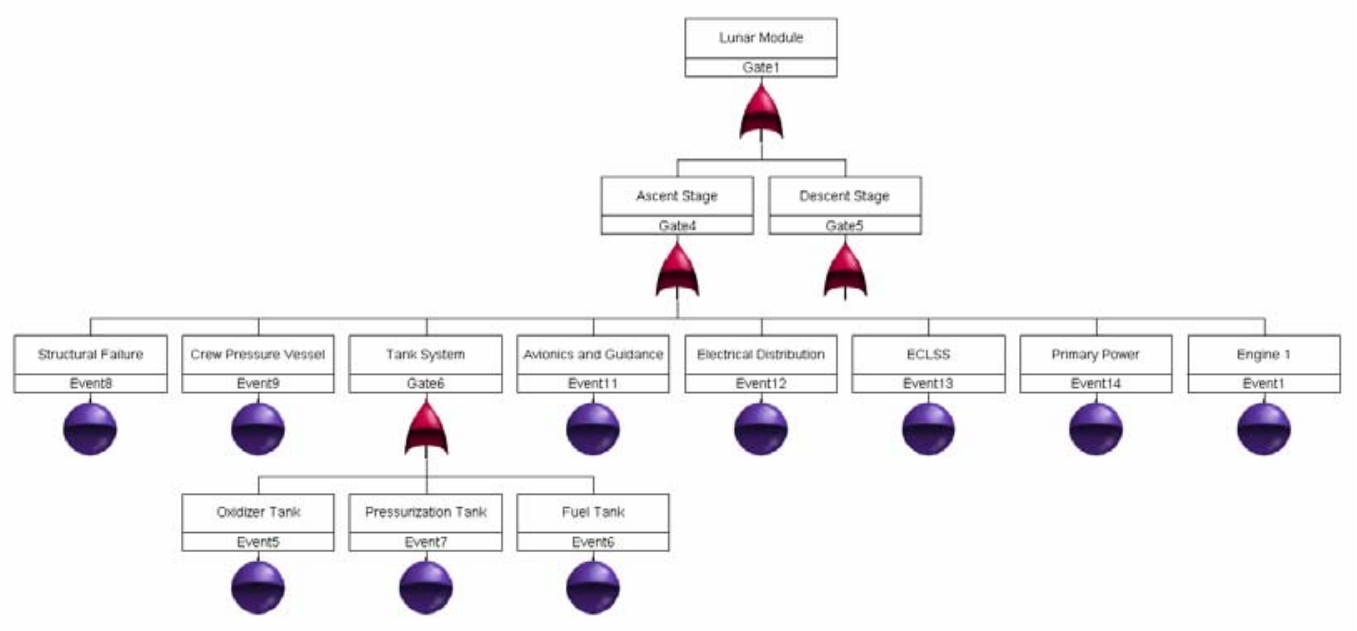

Figure 40: Fault Tree of Lunar Module

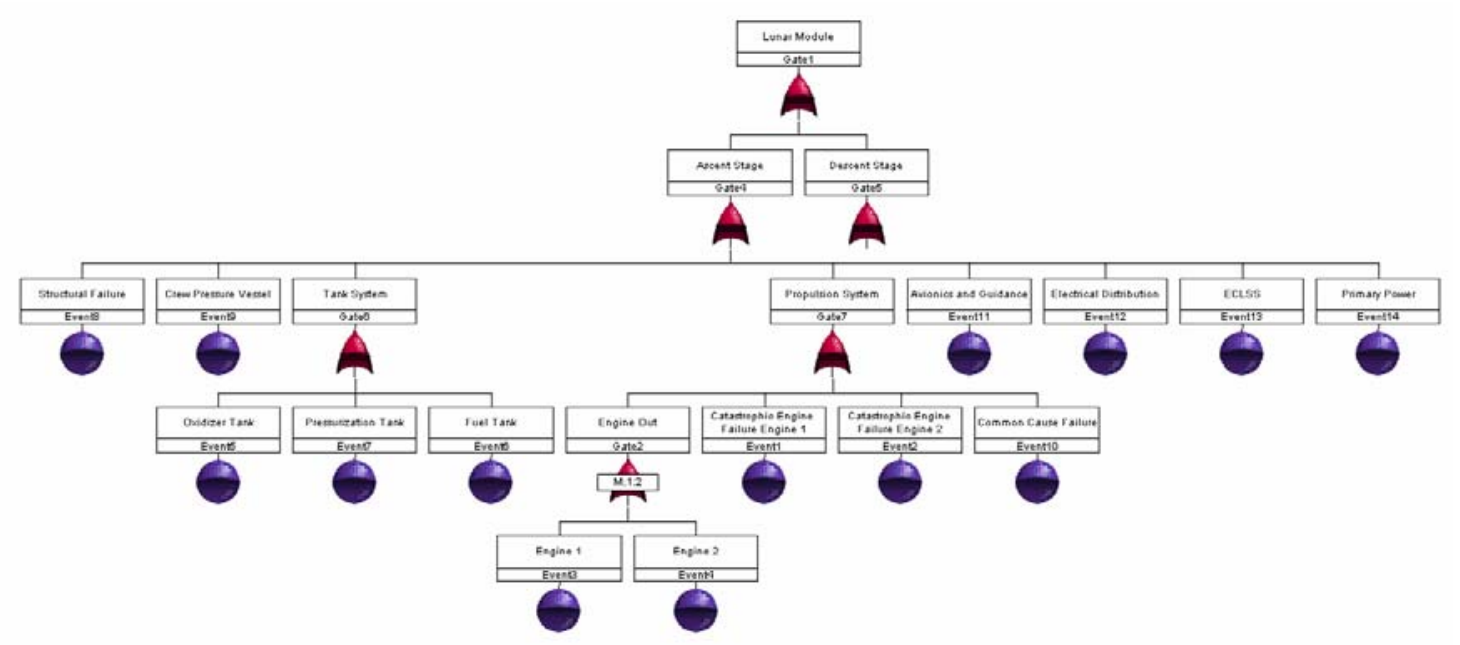

Figure 41: Fault Tree of Lunar Module with Engine Out Capability 
As these figures show adding a single engine out capability (a second engine to the operating engine) results in a drastic change to the fault tree. This is difficult and time consuming to set up all of the possible options in a fault tree analysis. The dynamically changing fault tree can adjust for redundancy (engine out) of any subsystem automatically in the spreadsheet tool.

To verify the spreadsheet tool, two discrete lunar module configurations were constructed in Relex [33]. Once confirmed with the fault tree tool the dynamically changing fault tree was integrated in the ROSETTA models. This model can accurately and quickly evaluate any vehicle configuration allowed by the performance models, including redundancy on any subsystem. A screenshot of the mathematical representation (spreadsheet) of the dynamically changing fault tree analysis is given as Figure 42 . 





This figure shows a screenshot of the spreadsheet model of the dynamically changing fault tree analysis. As this picture shows, each of the subsystem reliability inputs are adjusted by the redundancy and the catastrophic and common cause failure rates. The resulting loss of mission reliabilities and loss of crew safeties are then calculated for each ROSETTA model. These results are then passed to the architecture model to compile the lunar mission mode architecture reliability and safety. A comparison of the resulting reliabilities with the Apollo estimates is given in Chapter 4.

\subsection{Cost Estimation Using NAFCOM BASEd Cost Estimating RELATIONSHIPS}

To calculate the top level design discriminators, it is necessary to get an accurate cost model to predict the vehicle costs and compile architecture life cycle costs. The baseline cost models are cost estimating relationships described in Chapter 2. These CERs are weight based costing relationships that are based upon analogous systems to calculate the Design, Development, Testing, and Evaluation (DDT\&E) costs as well as the Theoretical First Unit (TFU) costs. These costs will then be compiled into a Life Cycle Cost (LCC) which will then be combined with the vehicle reliability to calculate the Pareto frontier which will be used to make the lunar architecture mode decision.

\subsubsection{Baseline NAFCOM Cost Estimating Relationships For lunaR}

\section{ARCHITECTURES}

As described in Chapter 2, the cost model used in the ROSETTA models is NAFCOM CER based. The $a$ and $b$ terms are derived from analogous vehicles which can be obtained from NAFCOM 04 for all of the Apollo vehicles [70]. These subsystem 
based costs are then compiled into a stage cost. This stage cost is then multiplied by NAFCOM relationships for Program Management, Integration, Assembly and Checkout, System Test Operations, Ground Support Equipment, and System Engineering and Integration. A cost margin of $20 \%$ is also applied to all vehicles which do not currently exist. The complexity factors for the CERs are all baselined at one and manipulated via the vehicle configuration choices and the cost penalties for using off-nominal reliabilities. These CERs are then linked with the weights calculation page in the ROSETTA models so that as the vehicle scales the costs automatically recalculate.

\subsubsection{COMPLEXITY FACTORS}

The complexity factor portion of the CERs is adjusted based upon the vehicle configuration. These complexity factors adjust for the fact that some materials are more expensive per pound than others. An example of this is that a tank made out of Titanium will weigh less than a tank made out of aluminum, but the titanium tank should cost more than the aluminum tank. A summary of the structural complexity factors is given as Table 14 [71].

Table 14: Complexity Factors for Tank Structural Materials

\begin{tabular}{|c|c|}
\hline Structure & $\begin{array}{c}\text { Complexity Factor } \\
\end{array}$ \\
\hline Al-Li & 1.5 \\
\hline $\mathrm{Ti}$ & 7 \\
\hline Graphite Epoxy Overwrapped & 5 \\
\hline MMC & 12 \\
\hline
\end{tabular}


As this table shows, the cost per pound of different spacecraft materials varies greatly. Aluminum is the cheapest and most widely used material. Graphite epoxy is currently estimated to be 5 times the price per pound of Al; however, the actual cost of a tank could be less than that of aluminum because the weight of the tank is significantly lighter due to the higher strength and lower density of graphite epoxy.

\subsubsection{COST ESTIMATION FOR OFF-NOMINAL RELIABILITIES}

Once the baseline costs are calculated, it is necessary to calculate the cost of off nominal reliabilities of subsystems. It is possible that the actual reliability may be higher or lower than the assumed reliability of the subsystem. If uncertainty is applied to the subsystem reliability, it is necessary to evaluate the costs of changing the reliabilities off of the baseline. To calculate the costs of off nominal reliabilities, a reliability cost model was formulated by the ReliaSoft Corporation [72]. This cost model assumes an exponential behavior of the cost of increasing the reliability of the system. The ReliaSoft model is given as equation 24 .

$$
C_{i}=\exp \left[(1-f)^{*}\left(\frac{R_{i}-R_{i, \text { min }}}{R_{i, \text { max }}-R_{i}}\right)\right]
$$

Where: $\mathrm{C}_{\mathrm{i}}$ is the subsystem cost

$f$ is the feasibility of increasing the subsystem reliability $\mathrm{R}_{\mathrm{i}}$ is the subsystem reliability $\mathrm{R}_{\mathrm{i}, \mathrm{min}}$ is the minimum reliability of the subsystem $\mathrm{R}_{\mathrm{i}, \max }$ is the maximum reliability of the subsystem 
The cost of increasing reliability is obtained from this model. Unfortunately this cost model does not take into account any information about the current state of the art reliability of the component. To account for this reliability, the exponential equation above was normalized by the exponential curve for the state of the art reliability. This results in a K-factor which can be applied to the CERs as a complexity factor to adjust the costs of increasing a component's reliability beyond the state of the art. This equation is given as equation 25 .

$$
K=\frac{\exp \left[(1-f) *\left(\frac{R_{i}-R_{i, \text { min }}}{R_{i, \max }-R_{i}}\right)\right]}{\exp \left[(1-f) *\left(\frac{R_{S O A}-R_{i, \min }}{R_{i, \max }-R_{S O A}}\right)\right]}
$$

Where: $\mathrm{K}$ is the complexity factor applied to the CER

$$
\mathrm{R}_{\mathrm{SOA}} \text { is the state of the art subsystem reliability }
$$

This model can then be directly applied to the CERs via equation 26 .

$$
\text { Cost }=K^{*} a(W)^{b}
$$

Where: $\mathrm{C}$ is the subsystem cost.

The resulting subsystem costs can be compiled and the total vehicle DDT\&E and TFU costs can be calculated. These vehicle costs can then be included into the lunar 
architecture selection tool to calculate the architecture Life Cycle Costs (LCC). A comparison of the resulting costs and the Apollo costs are given in Chapter 4.

\subsubsection{Life CyCle Cost CALCUlator}

Once the vehicle costs are calculated for each of the vehicles in the lunar architecture, the total life cycle costs for the architecture can be calculated. The life cycle cost calculator used in this thesis spreads the DDT\&E out over a specified number of years at the beginning of the program. This DDT\&E is spread as a Beta function over the specified number of years. Once the DDT\&E is paid, a flight rate is assumed and the cost per flight is calculated based upon the production costs for the elements. This production cost is calculated from the TFU costs and a learning curve rate as described in Chapter 2. This learning curve diminishes the cost of subsequent production vehicles because of the knowledge gained in producing the previous vehicle. For this research a learning curve rate of $90 \%$ was assumed. The top level inputs to the life cycle cost calculator are:

- Start year for the program

- Total number of years of the program

- Number of years to spread DDT\&E

- Flight rate (number of flights per year)

- Learning curve rate

From the top level data and the DDT\&E and TFU costs of the different architecture elements, a total life cycle cost can be calculated. A typical cost spreading for a lunar architecture is shown as Figure 43. 


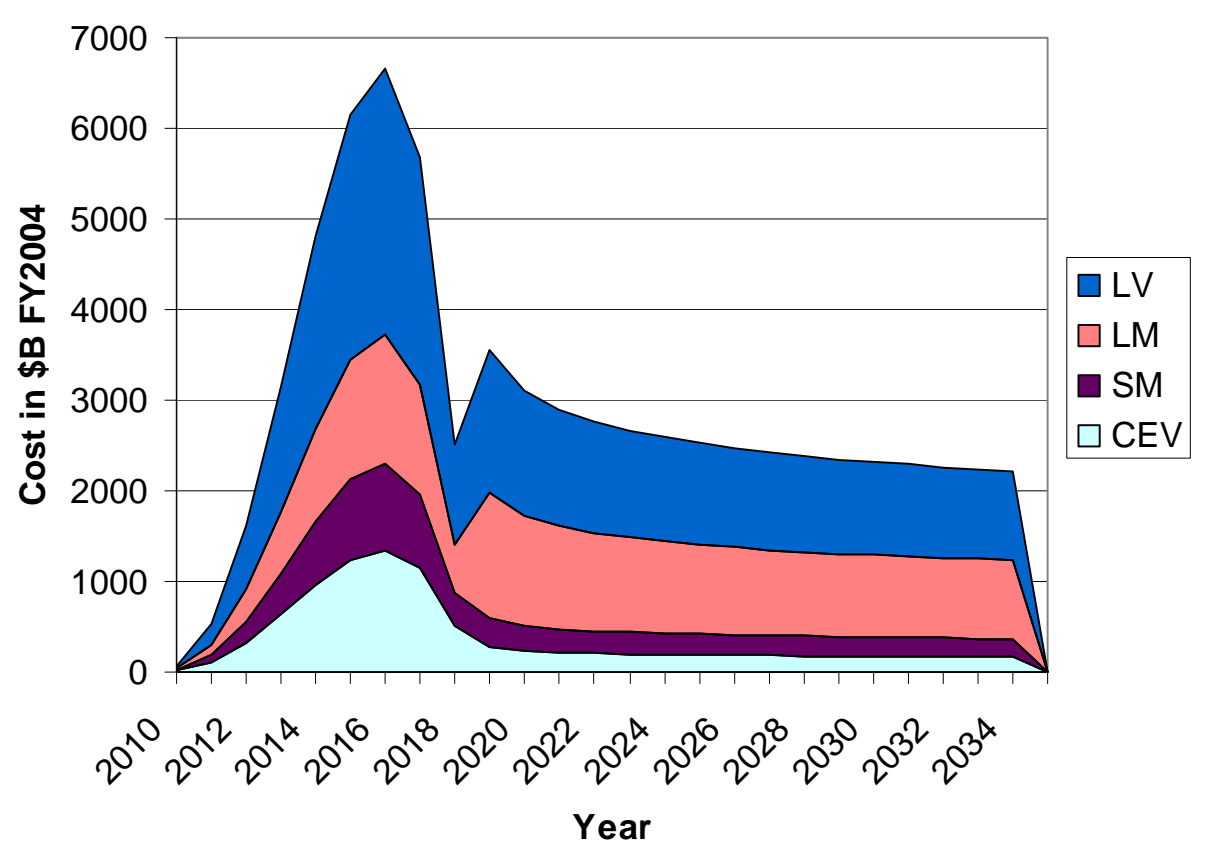

Figure 43: Life Cycle Cost Spreading for a Lunar Architecture

In this example, the four architecture elements' DDT\&E were spread over the first nine years of the program starting in 2010. The program continues for another 16 years to give a total program life of 25 years. For the 16 flight years, a flight rate of 2 flights per year is assumed. The resulting 32 flights are then compiled with the DDT\&E to calculate the total LCC. The production vehicles decrease in cost via the learning curve rate of $90 \%$. The total LCC is then used along with the loss of mission reliability to calculate the individual points on the Pareto frontier. This generic baseline mission profile (number of years, DDT\&E spreading, learning curve, and start year) will be used for all architecture comparisons made in this research.

The life cycle cost described in this methodology does not include an operations cost, nor does it include a disposal cost. The operations cost is highly dependant on the 
workforce and includes political aspects, both of which are difficult to quantify. The disposal cost is a small percentage of the LCC and is needed for all lunar architectures. Like the Apollo program and soon the space shuttle program, the program elements continue to be utilized long after the vehicle is retired. Because of these reasons the disposal cost is difficult to quantify and will not directly affect the lunar architecture decision.

\subsubsection{CASE STUdy-LUNAR Module ROSETTA Model CoSTING Model}

As with the reliability model described in the previous section, the cost model described above was implemented in the ROSETTA models included in the lunar architecture selection tool. The CERs are implemented in a spreadsheet along with the NAFCOM $a$ and $b$ coefficients to calculate the subsystem DDT\&E and TFU costs. The complexity factors for each of the subsystems are calculated as product of the original CER complexity factors calculated from the configuration type and the K-factor calculated from the reliability sheet. Each of these subsystem costs are then compiled to obtain the stage costs for the vehicle. These CERs are then linked with the weights calculated in the performance sections of the ROSETTA model to automatically update the subsystem costs as the vehicle scales.

The stage costs calculated from the NAFCOM relationships and the exponential cost-reliability model are then complied into an overall vehicle DDT\&E and TFU. The resulting DDT\&E and TFU are then passed to the architecture life cycle cost calculator to

compute the total LCC of the system. A screen shot of the spreadsheet implementation of the first stage of a LM cost model is included as Figure 44. 


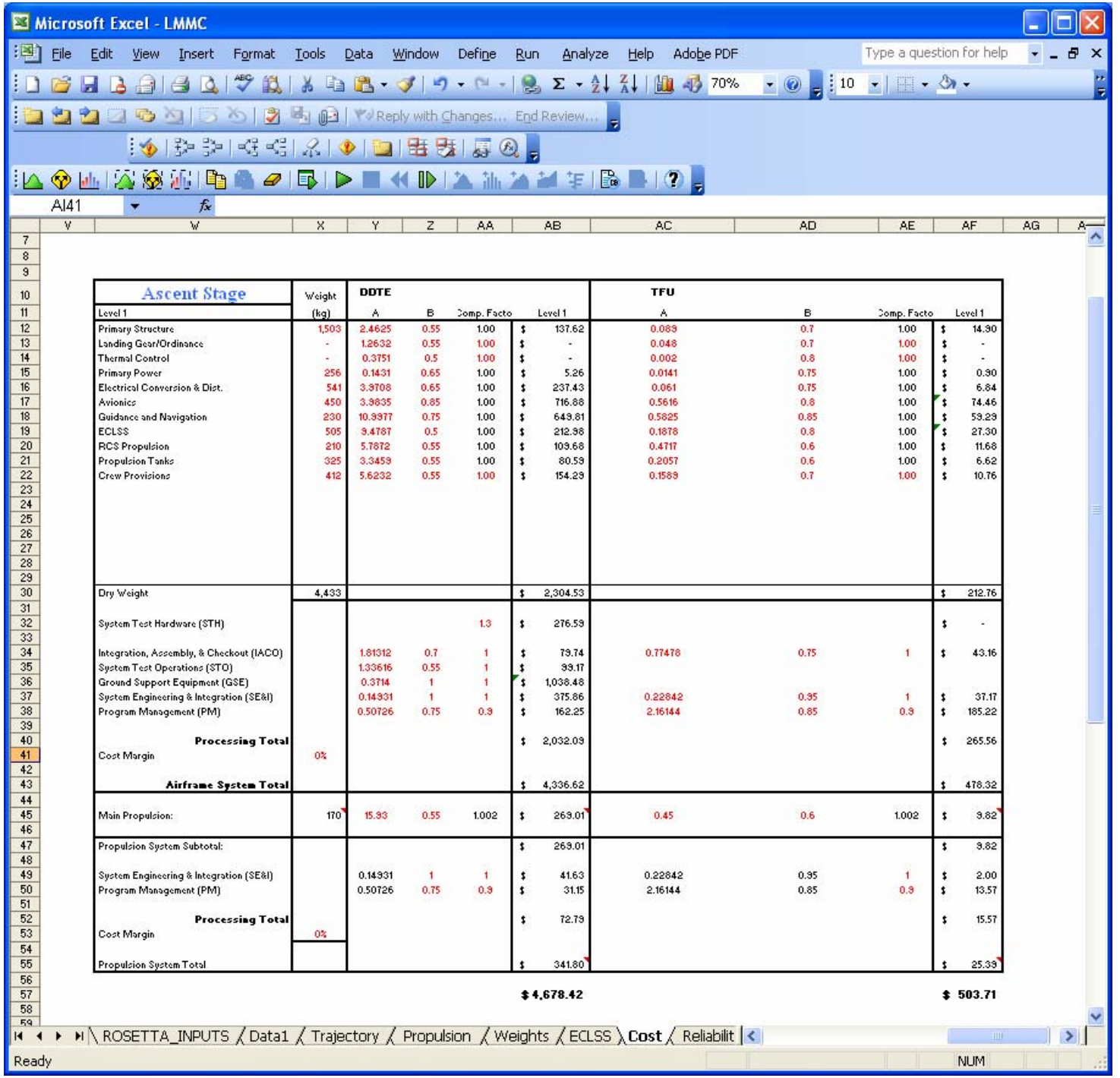

Figure 44: Screenshot of Lunar Module Cost Model 


\subsection{Implementation of ROSETTA Models In A LUNAR ARChitecture}

\section{SELECTION TOOL}

Once the ROSETTA models are completed for each of the vehicles required in the lunar mission mode, the vehicles must be integrated into a lunar architecture selection tool. To complete the lunar architecture DSM, the individual vehicle models must be included in an integrated framework to pass the vehicle mass, costs, and reliabilities between the elements of the architecture. ModelCenter is an integrated framework ideally set up to integrate multiple Excel based models and pass information between the models [73]. Each of the ROSETTA models were wrapped in ModelCenter wrappers and vehicle masses, costs, and reliabilities are then passed between the different ROSETTA models to complete the lunar architecture.

A separate inputs and cost accumulator module were also included in the integrated framework. The inputs module conditioned the vehicle level inputs so that the full lunar architecture is simulated. An example of this is that the Inputs page takes a user's selection of the lunar orbit rendezvous mission mode and sets the individual vehicles to perform the proper burns to complete the lunar orbit rendezvous. The cost accumulator module calculates the life cycle cost for the lunar architecture and performs the yearly cost spreading necessary to create Figure 43 . The resulting methodology is summarized in the flow chart presented as Figure 45. As this figure shows, the method involves a series of architecture inputs to be selected by the user. Once the mission mode and architecture inputs are selected, a Monte Carlo analysis randomly generates vehicle inputs which result in a design space exploration. The Pareto frontier is then taken from that design space exploration. 


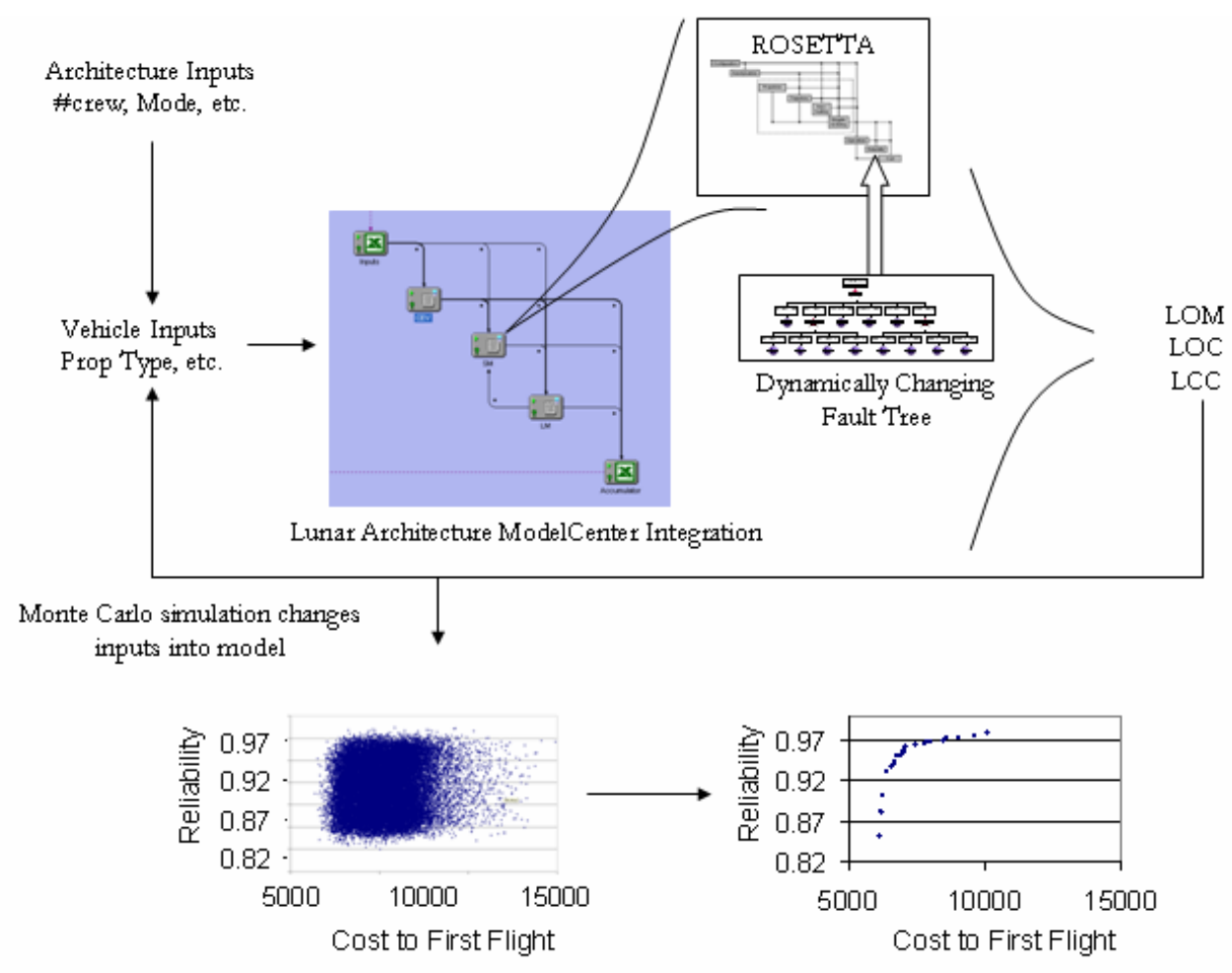

Figure 45: Monte Carlo Methodology of Lunar Architecture Selection

\subsubsection{OPTIMIZATION OF LUNAR VEHICLES}

The first design problem to be solved by this methodology is the lunar vehicle selection problem. For a given architecture mode, there are hundreds of thousands of different vehicle configurations that can accomplish the mission. Each of these vehicle configurations has an associated mass, cost, and reliability. To find the series of optimal Pareto solutions for the lunar vehicle a Monte Carlo analysis was completed for the vehicle ROSETTA model. This Monte Carlo analysis consisted of a series of 50,000 random sets of design variables created from a uniform distribution placed on each variable. The results of the Monte Carlo analysis for an Apollo-like lunar module are presented as Figure 46 and the Pareto frontier is presented as Figure 47. 


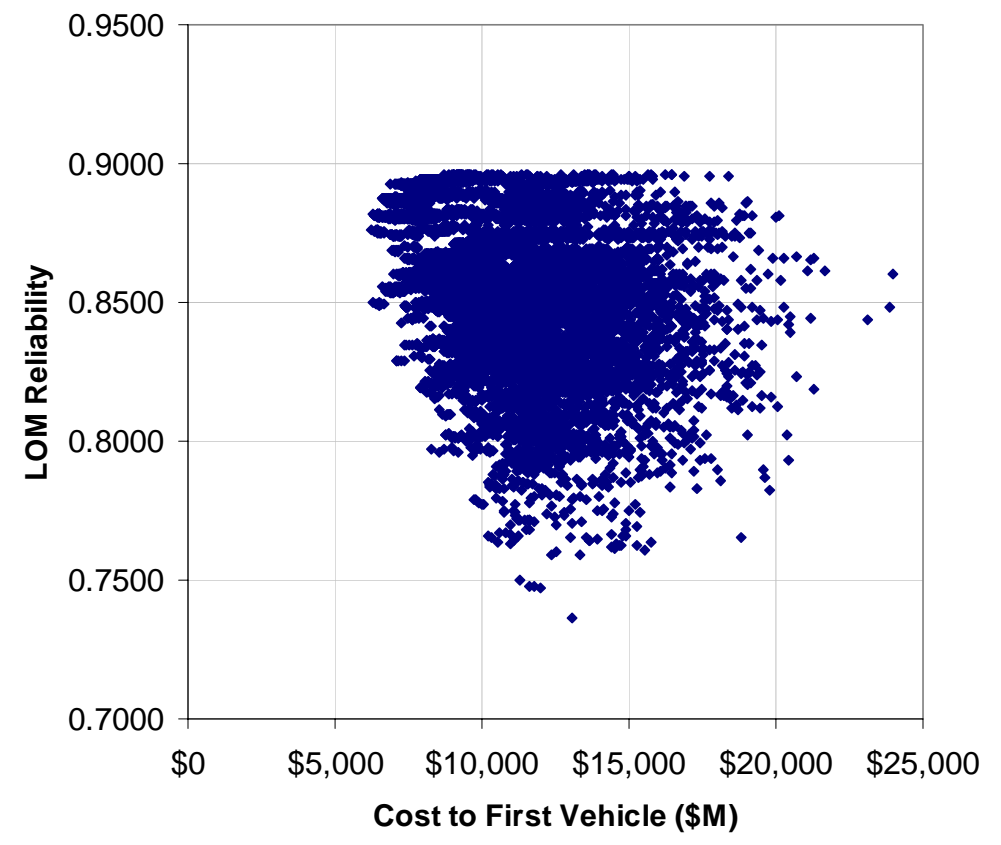

Figure 46: Monte Carlo Design Space Exploration of Apollo-like Lunar Module

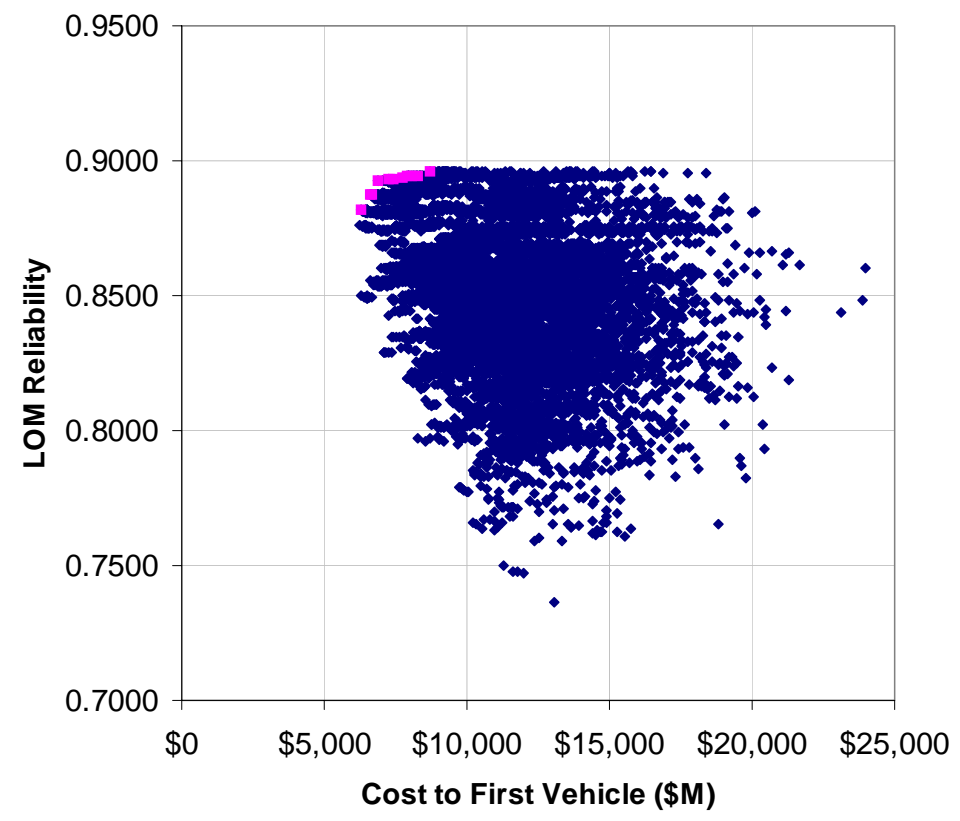

Figure 47: Pareto Frontier of Monte Carlo Design Space Exploration of Apollo-like Lunar Module 
The Pareto frontier created in Figure 47 depicts the optimal points from the design space exploration of the Apollo-like lunar module. As the number of design variables increases, the number of Monte Carlo simulation necessary to define the Pareto frontier increases exponentially. For a lunar module of 10 design variables, 50,000 runs is sufficient to define the design space. As the number of design variables increases to the 50 to 100 design variables necessary to define a lunar architecture, the Monte Carlo analysis exploration of the design space is no longer adequate. To investigate lunar architectures a Genetic Algorithm as described in Chapter 2 is applied to the lunar architecture framework. This integration of the GA and the lunar architecture model is shown as Figure 48.

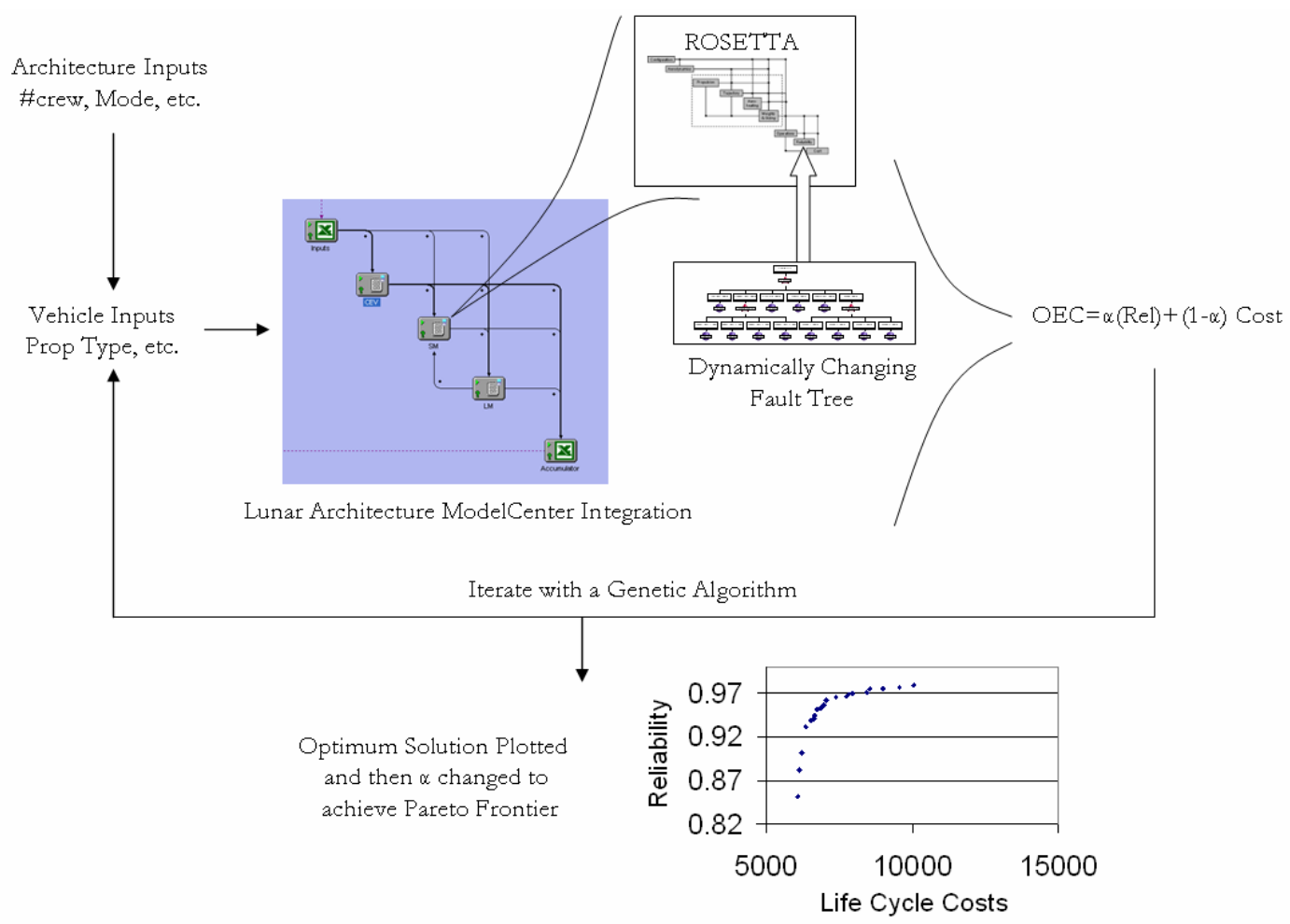

Figure 48: Integrated Lunar Architecture Selection Tool with Genetic Algorithm 
As this figure shows, the GA takes the place of the Monte Carlo analysis and an Overall Evaluation Criteria (OEC) is used as the objective function of the optimizer. This method is stochastic in that the initial population is randomly generated, but as a result of the higher objective function solution stay in the simulation longer, more points are evaluated around the Pareto frontier than a Monte Carlo analysis for a given number of simulations. The GA is solved for a given weighting $(\alpha)$ on the OEC. The OEC is given as equation 27.

$$
O E C=\alpha\left(\frac{R}{R_{B L}}\right)+(1-\alpha)\left(\frac{L C C_{B L}}{L C C}\right)
$$

The baseline LCC and R are different for the different lunar modes that are optimized. Each baseline is either the baseline numbers computed for the Apollo mission modes or the ESAS baseline. Each baseline was verified in Chapter 4 and then simulated using the generic lunar mission cost spreading shown in Figure 43. A summary of the LCC and reliability baselines is given as Table 15 .

Table 15: OEC Baseline Values for Each Lunar Mode

\begin{tabular}{lcc}
\hline Mission Mode & Baseline LCC & Baseline Reliability \\
Apollo LOR & $\$ 72,210 \mathrm{M}$ & 0.5245 \\
Apollo EOR & $\$ 96,392 \mathrm{M}$ & 0.3572 \\
Apollo Direct & $\$ 87,975 \mathrm{M}$ & 0.5315 \\
ESAS & $\$ 84,514 \mathrm{M}$ & 0.9427 \\
\hline
\end{tabular}


This method optimizes the Pareto frontier on an OEC constructed of loss of mission reliability and life cycle cost. This weighting $(\alpha)$ is then changed and the GA is rerun to find a second point on the Pareto frontier. The aforementioned procedure continues until enough points in the Pareto frontier are defined to adequately evaluate the Pareto frontier. A comparison of the Monte Carlo analysis and the GA implementation of the lunar architecture selection tool are given as Figure 49.

Figure 49 demonstrates the fact that the GA solves the Pareto frontier at least as accurately as the Monte Carlo analysis. In fact the GA finds slightly better solutions. This is a result of the fact that the GA is evaluating more points around the solution that the purely random Monte Carlo analysis. The GA also found the Pareto frontier in approximately 25,000 function calls. This was completed in half the time it took the Monte Carlo analysis to produce a less efficient Pareto frontier. 


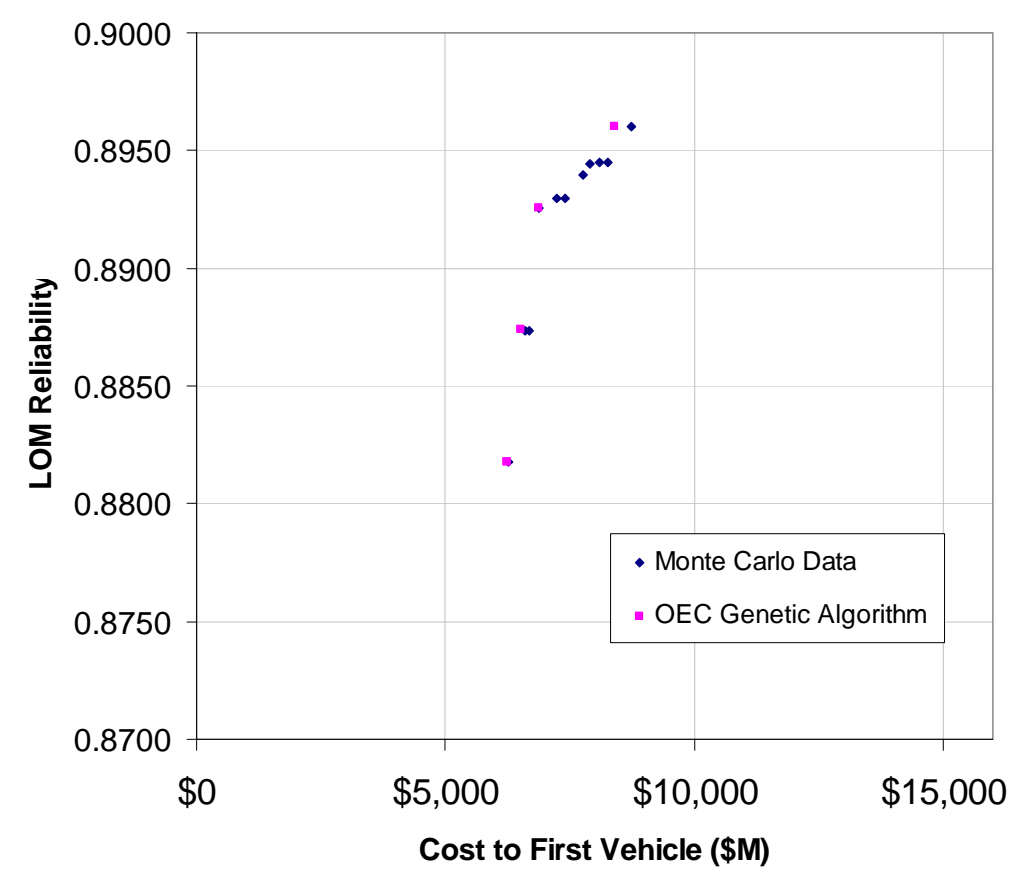

Figure 49: Comparison of Monte Carlo Analysis and Genetic Algorithm for an Apollolike Lunar Module

The given method of defining the Pareto frontier through the use of a GA will be implemented in all subsequent lunar architecture selection problems and the resulting Pareto frontiers will then be used by the decision maker to define the ideal lunar architecture for a given LCC.

\subsection{IMPLEMENTATION OF UNCERTAINTY IN LUNAR ARCHITECTURE} SELECTION TOOL

Once the methodology of optimizing a lunar architecture is set, it is necessary to address the uncertainty in the individual vehicle ROSETTA models. As noted in Chapter 2, the uncertainty in the ROSETTA models will be addressed with a Monte Carlo analysis. The Monte Carlo analysis will be conducted by applying triangular 
distributions to the subsystem reliabilities and the catastrophic engine fractions in each of the ROSETTA models. The range of the triangular distributions will be set to be 90 $110 \%$ of the baseline failure rates. The range of the catastrophic engine failure rates will be set to $10-20 \%$. Once these distributions are set each optimized Pareto points will be evaluated through a 10,000 run Monte Carlo simulation to address the uncertainty associated with reliability of the architecture.

As noted in Section 3.3.3, the cost of off-nominal reliabilities will also be addressed. As the Monte Carlo analysis changes the subsystem reliabilities, the cost model will also adjust the vehicle costs to take into account the increase or decrease in reliability. The result will be a Probability Density Function (PDF) on the cost and reliability of the system. Once this PDF is defined, it can be integrated and the Cumulative Distribution Function (CDF) can be found. Once this CDF is created error bars can be applied to the resulting Pareto frontiers to get a $10 \%$ and $90 \%$ confidence band. This resulting band will define the limits (best and worst case scenarios) of the lunar architecture mode. These best and worst case scenarios can then be compared much like the Pareto frontiers are compared to find the best lunar architecture for a any given budget. 


\section{CHAPTER 4}

\section{VALIDATIONS OF LUNAR ARCHITECTURE SIMULATION}

Chapter 3 described the method that is used to supply the decision maker with the ability to choose different optimized lunar architectures based upon the high level design discriminators such as cost, reliability, and safety. This method is then applied to different baseline lunar architecture modes to determine the Pareto frontier for each of these modes. The Pareto frontiers supply the decision maker with information about the most reliable architecture selections for changing budgets (life cycle costs) of the lunar program. To baseline this technique, the three Apollo modes that were under consideration in the 1960s (Earth Orbit Rendezvous, Lunar Orbit Rendezvous, and Lunar Direct Missions) were simulated and compared with the solutions obtained in the Apollo Program. To baseline the models with current vehicle design techniques; the Exploration System Architecture Study (ESAS) (EOR-LOR) mission mode was also simulated and compared with the baseline values. Once the procedure is verified, it was used to calculate the optimal Pareto frontiers for each of the mission modes.

To verify this design methodology and to validate the accuracy of the ROSETTA models, each of the mission modes under consideration was simulated and compared with the solutions obtained using the conventional conceptual design techniques. To accomplish this simulation a complete ROSETTA model was created for each of the vehicles in the lunar architectures. These ROSETTA models were individually validated 
and then compiled into a lunar architecture using a ModelCenter integration environment [73]. This ModelCenter based simulations will then be used to compute the performance, cost, reliability, and safety for each of the lunar mission modes. These results (performance, cost, and reliability) are then compared with the results from a conventional conceptual design process to validate the method and the results of each of the lunar architecture simulations.

\subsection{Apollo Lunar Orbit Rendezvous Baseline}

Perhaps the most recognizable mission mode for lunar exploration is the Apollo Lunar Orbit Rendezvous mission mode. The LOR mission mode was originally championed by John Houbolt, a NASA Langley engineer [7]. This mission mode involved the use of a single Saturn V (C-5) launch vehicle to launch a three person Command Module (CM), a hypergolic pressure fed service module (SM), and a two person, two stage hypergolic pressure fed Lunar Module (LM). This mission mode is demonstrated in Figure 50.

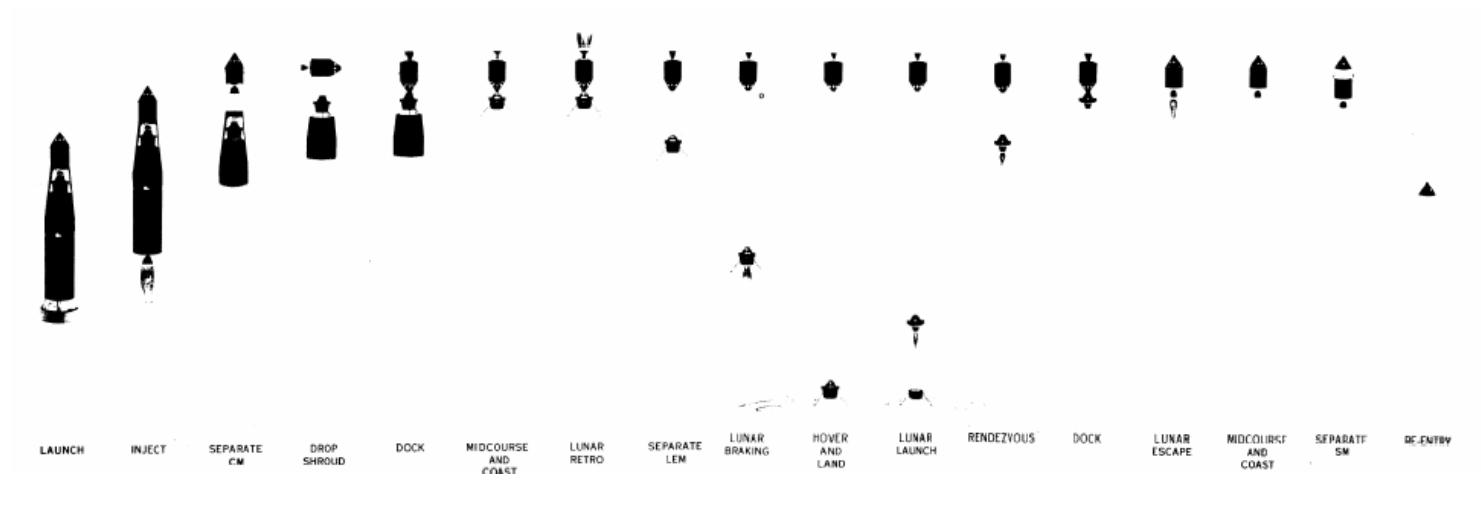

Figure 50: Lunar Orbit Rendezvous Mission Mode [4]. 
As this figure depicts, the Apollo LOR concept involves a single launch of a Saturn V launch vehicle. The Saturn V is a three stage launch vehicle consisting of an S-I C first stage, and S-II second stage and an S-IV B third stage. The three man crew resides in the Command Module (CM) during the ascent. The $\mathrm{CM}$ is an Aluminum capsule designed to carry the crew to the low lunar orbit and return them to the Earth. Once in Low Earth Orbit (LEO) the S-IV-B performs a burn (Trans Lunar Injection (TLI)) to propel the vehicle stack to the Moon. The Service Module (SM) enters the stack (Lunar Module (LM) and CM) into Low Lunar Orbit (LLO). The Lunar Module is designed to take two men (via a separate habitat) to the lunar surface from low lunar orbit. The third man remains in the $\mathrm{CM}$ for the duration of the lunar surface mission. The LM is a two stage vehicle that uses common propellants as the SM. The staging point for the LM is at the lunar surface. The Lunar Ascent Module then returns the two man lunar crew to LLO and rendezvous with the $\mathrm{CM}$ and the SM. The SM propels the CM to earth by performing the trans-earth injection (TEI) burn. The CM subsequently enters the Earth's atmosphere and descends via parachutes for a water touch down. A compilation of the design choices made in the Apollo LOR mission is given below as Table 16 and Table 17.

Table 16: Propellant Choices for Apollo LOR.

\begin{tabular}{lccc}
\hline Vehicle & Propellants & Engine & Engine Number \\
S-I-C & LOX/RP1 & F-1 (Gas Generator) & 5 \\
S-II & LOX/LH2 & J-2 (Gas Generator) & 5 \\
S-IV B & LOX/LH2 & J-2 (Gas Generator) & 1 \\
SM & UDMH/N204 & Pressure Fed & 1 \\
LM Descent & UDMH/N204 & Pressure Fed & 1 \\
LM Ascent & UDMH/N204 & Pressure Fed & 1 \\
\hline
\end{tabular}


Table 17: Structure Choices for Apollo LOR.

\begin{tabular}{lccc}
\hline Structure & Main Tanks & Pressurant Tanks & Other Structure \\
S-I-C & $\mathrm{Al}$ & $\mathrm{Ti}$ & $\mathrm{Al}$ \\
S-II & $\mathrm{Al}$ & $\mathrm{Ti}$ & $\mathrm{Al}$ \\
S-IV B & $\mathrm{Al}$ & $\mathrm{Ti}$ & $\mathrm{Al}$ \\
CM & $\mathrm{NA}$ & $\mathrm{Al}$ & $\mathrm{Al}$ \\
SM & $\mathrm{Al}$ & $\mathrm{Ti}$ & $\mathrm{Al}$ \\
LM Descent & $\mathrm{Al}$ & $\mathrm{Ti}$ & $\mathrm{Al}$ \\
LM Ascent & $\mathrm{Al}$ & $\mathrm{Ti}$ & $\mathrm{Al}$ \\
\hline
\end{tabular}

It is important to note that the Apollo LOR mission mode assumes the same engines for the second and third stages of the Saturn V. This is important because the DDT\&E for this engine can be shared by both stages. The same is true for the LM descent engine and the SM engine. These engines used the same propellant and are approximately the same thrust levels so a cost benefit also occurs.

The Apollo LOR was simulated in ModelCenter using ROSETTA models and the methods described in Chapter 3. The simulation involved the creation of ROSETTA models for the Command Module, the Service Module, the Lunar Module, and the Saturn V. These ROSETTA modules were then wrapped in ModelCenter to integrate the performance characteristics of the mission mode. The Architecture Cost Model (ACM) was also integrated to compile the costs of the different vehicles can then calculate the total Life Cycle Costs (LCC) of the mission mode.

Each of the ROSETTA models were constructed using Response Surface Methods for performance calculations, NAFCOM based cost estimations, and dynamically changing fault trees for the reliability analysis. The original subsystem reliabilities for the fault tree analyses were derived from the Apollo Reliability and 
Quality Assurance Program Quarterly Status Report [69]. These reliabilities were compiled in the dynamic fault trees to calculate the Loss of Mission (LOM) and Loss of Crew (LOC) values for each of the ROSETTA models. A screen shot of the ModelCenter tool is given as Figure 51.

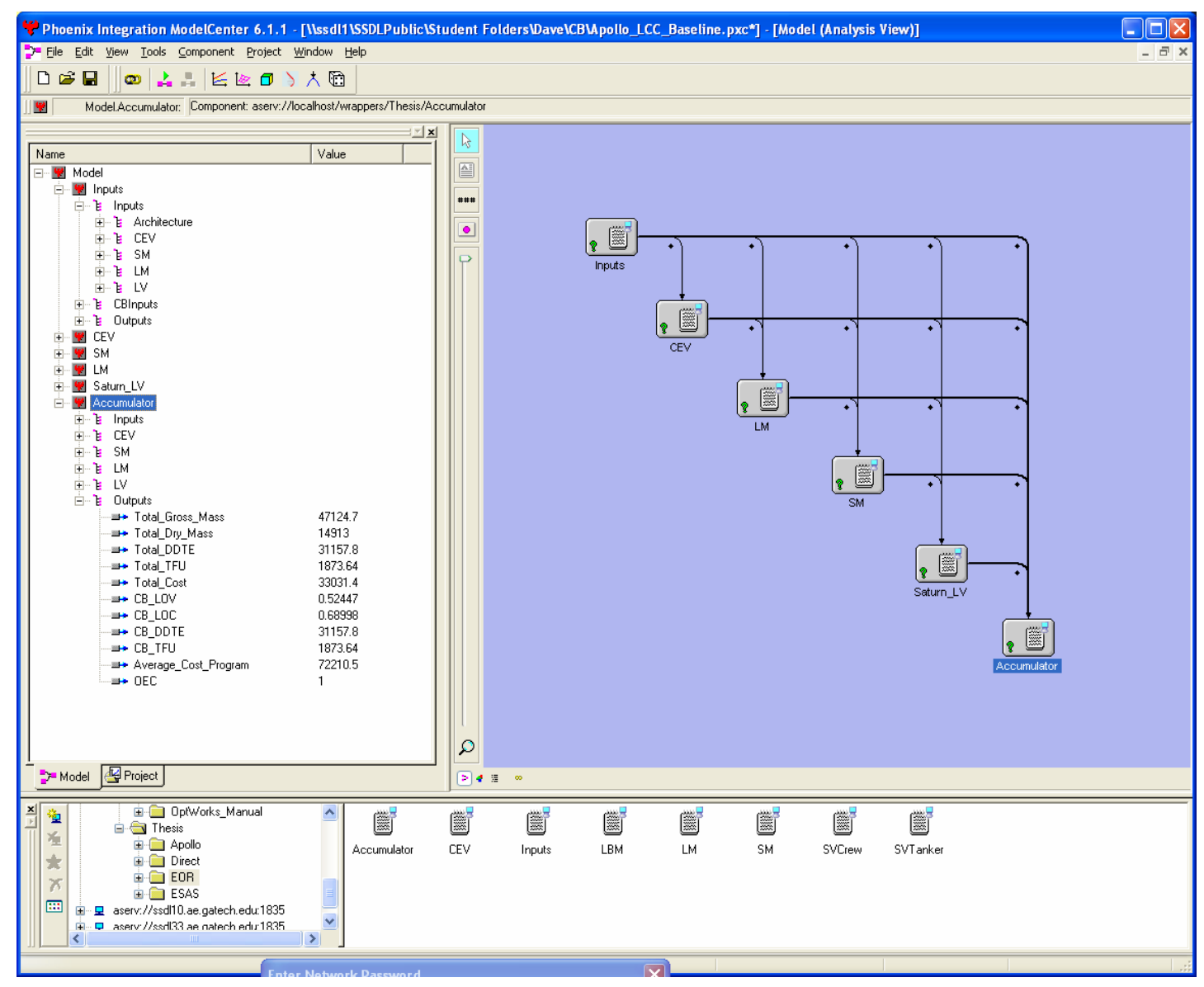

Figure 51: ModelCenter Based Lunar Orbit Rendezvous Simulation [73].

As this figure demonstrates, ModelCenter treats the design problem like a Design Structure Matrix (DSM). Each of the contributing analyses is a vehicle in the lunar architecture. The first box is a program to condition the inputs so that a single model can be used to evaluate each of the mission modes with minimal model changes. The final 
box is the Architecture Cost Model which compiles the performance, costs, and reliabilities and applies the cost spreading over the life of the architecture to obtain the Life Cycle Cost. The Apollo LOR DSM contributing analysis order is set up so the architecture can be computed as a completely feed-forward system. The resulting simulation has 83 design variables and each closed architecture can be computed in less than five seconds.

The Apollo LOR baseline was computed using this ROSETTA based simulation tool. The 83 design variables were set and the ROSETTA models were evaluated to close the Apollo LOR mission mode. The performance results were compared between the simulation results and the actual Apollo LOR vehicle weights [74]. The results are given as Table 18.

Table 18: Comparison of Apollo LOR Performance Results.

\begin{tabular}{lccc}
\hline & Apollo Baseline & ROSETTA Models & $\%$ Diff \\
CM & $5,922 \mathrm{~kg}$ & $6,024 \mathrm{~kg}$ & $1.72 \%$ \\
SM & $24,721 \mathrm{~kg}$ & $24,917 \mathrm{~kg}$ & $0.79 \%$ \\
LM & $16,437 \mathrm{~kg}$ & $16,183 \mathrm{~kg}$ & $-1.54 \%$ \\
Stack & $47,079 \mathrm{~kg}$ & $47,124 \mathrm{~kg}$ & $0.09 \%$ \\
S-I-C & $2,278,712 \mathrm{~kg}$ & $2,279,540 \mathrm{~kg}$ & $0.04 \%$ \\
S-II & $483,951 \mathrm{~kg}$ & $477,181 \mathrm{~kg}$ & $-1.40 \%$ \\
S-IV & $122,723 \mathrm{~kg}$ & $128,378 \mathrm{~kg}$ & $4.61 \%$ \\
CES & $4,161 \mathrm{~kg}$ & $4,106 \mathrm{~kg}$ & $-1.32 \%$ \\
On Pad & $2,936,627 \mathrm{~kg}$ & $2,936,329 \mathrm{~kg}$ & $-0.01 \%$ \\
\hline
\end{tabular}

As this table shows, the mass results for the ROSETTA models are within 5\% of the actual vehicle weights. The biggest discrepancy is on the Saturn IV-B stage. This large discrepancy may be due to the fact that any errors in the stack weight are compounded in 
the TEI stage since it must perform the large delta $V(\sim 10,000 \mathrm{ft} / \mathrm{s})$ required for that burn. The entire stack weight is within $0.01 \%$ of the actual mass of the Saturn V vehicle at the pad. These mass estimations are very accurate and have to do with the fact that very few manned space vehicles have been built and the MERs used in the ROSETTA models have been based upon this limited vehicle set which includes the Apollo vehicles.

The mass estimates for the Apollo LOR mission mode are very accurate; and therefore, a mass-based cost comparison can be made between the Apollo architecture costs and the ROSETTA model calculated costs. As noted previously, these Cost Estimating Relationships (CERs) are bottoms-up subsystem based costing methods. These NAFCOM based CERs scale according to the mass of the subsystems and the complexity of the subsystem as compared with the baseline. The resulting simulation costs are then compared with the NAFCOM published cost for the Apollo vehicles. The comparison of Design Development Testing \& Evaluation (DDT\&E) is given as Table 19 and the Theoretical First Unit (TFU) costs are given as Table 20.

Table 19: Apollo LOR Design Development Testing and Evaluation Cost Comparison.

\begin{tabular}{llll}
\hline$\$ M 2004$ & Apollo & ROSETTA Models & $\%$ Diff \\
CSM & $\$ 10,505 \mathrm{M}$ & $\$ 10,773 \mathrm{M}$ & $2.55 \%$ \\
LM & $\$ 6,752 \mathrm{M}$ & $\$ 6,639 \mathrm{M}$ & $-1.67 \%$ \\
Saturn V & $\$ 13,887 \mathrm{M}$ & $\$ 13,744 \mathrm{M}$ & $-1.03 \%$ \\
Total DDTE & $\$ 31,144 \mathrm{M}$ & $\$ 31,156 \mathrm{M}$ & $0.04 \%$ \\
\hline
\end{tabular}


Table 20: Apollo LOR Theoretical First Unit Cost Comparison.

\begin{tabular}{llll}
\hline \$M 2004 & Apollo & ROSETTA Models & $\%$ Diff \\
CSM & $\$ 294 \mathrm{M}$ & $\$ 309 \mathrm{M}$ & $5.10 \%$ \\
LM & $\$ 651 \mathrm{M}$ & $\$ 733 \mathrm{M}$ & $12.60 \%$ \\
Saturn V & $\$ 901 \mathrm{M}$ & $\$ 832 \mathrm{M}$ & $-7.71 \%$ \\
Total TFU & $\$ 1,846 \mathrm{M}$ & $\$ 1,874 \mathrm{M}$ & $1.49 \%$ \\
\hline
\end{tabular}

As these tables show, the costs are very close for each of the vehicles in the Apollo LOR. DDT\&E costs are within 3\% for all of the elements of the architecture. The error associated with the TFU costs is slightly higher. The main difference is in the TFU of the LM. This higher cost of $12 \%$ results in a difference of $\$ 82$ million dollars. Even with this difference, the total TFU is only off by $1.49 \%$, or $\$ 8$ million dollars, for the first vehicle. The ROSETTA calculated cost to first vehicle is $\$ 33.03$ Billion which is less than $0.1 \%$ off the actual cost to first vehicle of $\$ 32.99$ Billion.

The dynamically changing fault tree analysis incorporated in each ROSETTA model calculates the total vehicle reliability based upon the assumed subsystem reliabilities and the vehicle configurations. The reliability analysis was conducted for each of the ROSETTA models in the Apollo LOR, and the results were compared with those published in the Apollo Reliability and Quality Assurance Program Quarterly Status Report [69]. The resulting reliability comparisons are given in Table 21. 
Table 21: Apollo LOR Loss of Mission Reliability Comparison.

\begin{tabular}{llll}
\hline & Apollo & ROSETTA Models & \% Diff \\
CSM & 0.7662 & 0.7648 & $-0.19 \%$ \\
LM & 0.8894 & 0.8918 & $0.27 \%$ \\
Stack & 0.6815 & 0.6820 & $0.08 \%$ \\
Saturn V & 0.7639 & 0.7690 & $0.66 \%$ \\
LOM & 0.5206 & 0.5245 & $0.75 \%$ \\
\hline
\end{tabular}

As this table shows, the resulting Apollo LOR reliability calculations are within 1\% of the actual calculated Apollo LOR LOM reliabilities. This high level of accuracy is achieved because of the subsystem reliabilities are derived from the actual Apollo numbers. The total calculated LOM is 0.5245 , or a loss of mission in every 2.10 flights. The demonstrated LOM of the Apollo LOR system is 1 loss in 7 flights or 0.87. For comparison purposes, the different lunar mode reliabilities will be based upon the calculated reliabilities and not the demonstrated reliabilities of the LOR.

The ROSETTA model accurately calculates the performance, cost, and reliability of the Apollo LOR mission mode. These ROSETTA models, combined in the ModelCenter environment, will then be used to find the Pareto frontiers of the ideal reliabilities for the varying budgets of the LOR program.

\subsection{Apollo Direct Mission Mode BASELINE}

The Apollo Direct (DIR) is a second mission mode considered by the Apollo project. This mission mode was actually the first considered by NASA and requires the largest vehicles. This mission mode involved the use of a large Saturn derived NOVA launch vehicle. This massive launch vehicle would launch a vehicle stack which would take three men to the surface of the Moon and return them to Earth. The Nova launch 
vehicle launches a three person Command Module (CM), a hypergolic pressure fed service module (SM), a LOX/LH2 expander cycle Lunar Breaking Module (LBM) and a stage hypergolic pressure fed Lunar Touch Down Module (LTDM). This mission mode is demonstrated in Figure 52.

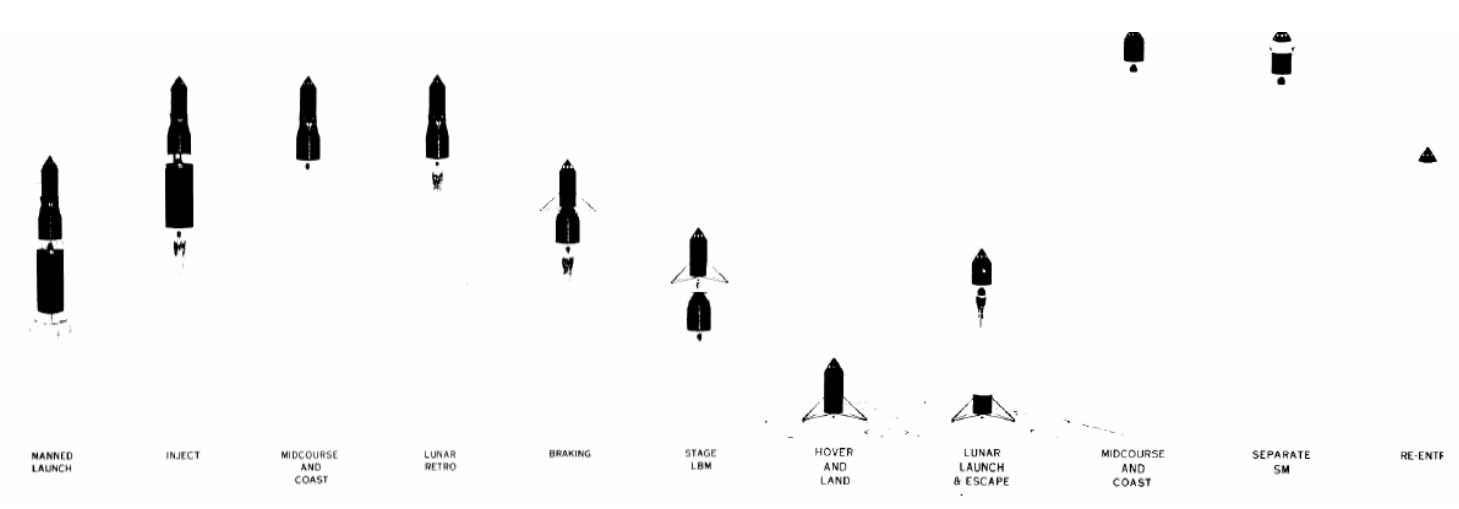

Figure 52: Direct Mission Mode [4].

As this figure depicts, the Direct mission concept involves a single launch of a Nova launch vehicle. The Nova launch vehicle is a three stage launch vehicle derived from the Saturn V launch vehicle. The Nova vehicle has 8 F-1 derived engines and $8 \mathrm{~J}-2$ derived engines on the second stage. The third stage of the Nova vehicle is similar to the S-IV-B stage with one $\mathrm{J}-2$ derived engine. The $\mathrm{CM}$ is similar to the $\mathrm{CM}$ used by the Apollo LOR in that it is a three man Aluminum capsule. Once in Low Earth Orbit (LEO), the third stage of the NOVA performs a burn (Trans Lunar Injection (TLI)) to propel the stack to the Moon. The LBM slows the vehicle stack at the moon and descends the stack towards the lunar surface. The LBM then stages and the LTDM hovers and lands the entire stack (CM and SM) on the lunar surface. Finally, the CEV acts as the lunar habitat on the lunar 
surface and the SM provides the power for the surface missions. After the lunar surface mission the SM performs the ascent from the lunar surface and the TEI burn. The CM then directly enters the Earth's atmosphere and descends via parachutes for a water touch down. A compilation of the design choices made in the Apollo Direct mission is given below as Table 22 and Table 23.

Table 22: Propellant Choices for Apollo Direct.

\begin{tabular}{lccc}
\hline Vehicle & Propellants & Engine & Engine Number \\
Nova $^{\text {st }}$ Stage & LOX/RP1 & F-1 Derived (Gas Generator) & 8 \\
Nova 2 ${ }^{\text {nd }}$ Stage & LOX/LH2 & J-2 Derived (Gas Generator) & 8 \\
Nova ${ }^{\text {rd }}$ Stage & LOX/LH2 & J-2 Derived (Gas Generator) & 1 \\
SM & UDMH/N204 & Pressure Fed & 1 \\
LBM & LOX/LH2 & Expander & 1 \\
LTDM & UDMH/N204 & Pressure Fed & 1 \\
\hline
\end{tabular}

Table 23: Structure Choices for Apollo Direct.

\begin{tabular}{lccc}
\hline Structure & Main Tanks & Pressurant Tanks & Other Structure \\
Nova $1^{\text {st }}$ Stage & $\mathrm{Al}$ & $\mathrm{Ti}$ & $\mathrm{Al}$ \\
Nova $2^{\text {nd }}$ Stage & $\mathrm{Al}$ & $\mathrm{Ti}$ & $\mathrm{Al}$ \\
Nova ${ }^{\text {rd }}$ Stage & $\mathrm{Al}$ & $\mathrm{Ti}$ & $\mathrm{Al}$ \\
CM & $\mathrm{NA}$ & $\mathrm{Al}$ & $\mathrm{Al}$ \\
SM & $\mathrm{Al}$ & $\mathrm{Ti}$ & $\mathrm{Al}$ \\
LBM & $\mathrm{Al}$ & $\mathrm{Ti}$ & $\mathrm{Al}$ \\
LTDM & $\mathrm{Al}$ & $\mathrm{Ti}$ & $\mathrm{Al}$ \\
\hline
\end{tabular}

As with the Apollo LOR mission mode, the Apollo Direct mission mode assumes the same engines for the second and third stages of the launch vehicle. This is important because the DDT\&E for this engine can be shared by both stages. It is interesting to note 
that the LBM uses RL-10 based LOX/LH2 expander cycle engines. This propellant choice is made to limit the weight of the launched vehicle stack

As with the Apollo LOR mission mode, the Direct mission was simulated in ModelCenter using ROSETTA models and the methods described in Chapter 3. The simulation involved the use of the same ROSETTA models as Apollo LOR for the Command Module, the Service Module, the Lunar Module, and the Saturn V. These ROSETTA models were made generic enough to change the burn structure in the models so that the same model could accomplish both mission modes. The main differences between the modes are that the $\mathrm{CM}$ had to be adapted to travel to the lunar surface, The LM descent stage acts as only a LTDM, eliminating the ascent stage and the crew compartment, and a LBM had to be added to the architecture. These ROSETTA modules were then wrapped in ModelCenter to integrate the performance characteristics of the mission mode. The Architecture Cost Model (ACM) was modified to accumulate the mass, cost, and reliabilities of all of the architecture vehicles to calculate the total Life Cycle Costs (LCC) of the mission mode.

As with the Apollo LOR mission mode, the subsystem reliabilities for the Direct mission modes were derived from the Apollo Manned Lunar Landing Program Mode Comparison [75]. These reliabilities were compiled in the dynamic fault trees to calculate the Loss of Mission (LOM) and Loss of Crew (LOC) values for each of the ROSETTA models. A screen shot of the Direct mission mode ModelCenter tool is given as Figure 53. 


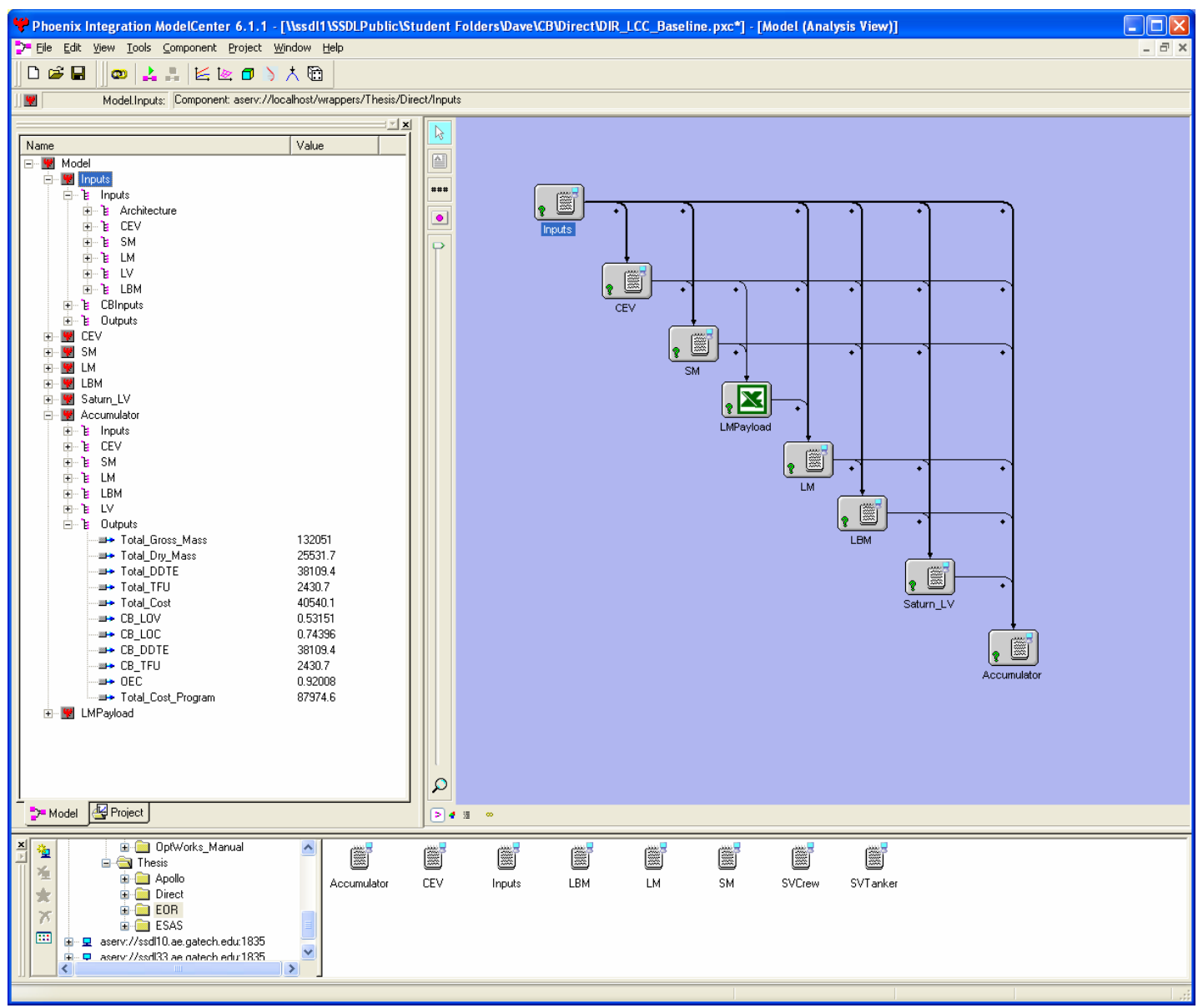

Figure 53: ModelCenter Based Direct Mission Simulation [73].

As this figure depicts, the Direct mission mode simulation is very similar to the Apollo LOR. The main differences are the order of the contributing analyses and introduction of a LBM. As with the Apollo LOR model the first box is a program to condition the inputs. This is important for the Direct mission mode since this contributing analysis changes the model inputs so that the individual vehicles perform the appropriate burns of the architecture. The final box is the Architecture Cost Model which compiles the performance, costs, and reliabilities and applies the cost spreading over the life of the architecture to get the Life Cycle Cost. The Direct DSM contributing analysis order is 
setup so the architecture can be computed as a completely feed-forward system. For the Direct mode the SM can be evaluated before the LM because in this mode the LM requires the mass of the SM to size the vehicle. In the Apollo LOR the LM mass must be calculated before the SM since the SM performs the LOI burn with the LM as payload. The LBM is calculated just before the launch vehicle since the LBM requires the closed masses of the CM, SM, and LTDM, since those elements are payloads for the LBM. The resulting simulation has 96 design variables and each closed architecture can be computed in less than five seconds.

The Apollo Direct baseline was computed using this ROSETTA based simulation tool. The 96 design variables were set and the ROSETTA models were evaluated to close the Apollo Direct mission mode. Because of the limited data availability of the actual Apollo Direct modes comparison and the similarity of the Apollo Direct models to the Apollo LOR models, a separate validation of the Apollo Direct results was not simulated.

Table 24: Comparison of Apollo Direct to Apollo LOR Performance.

\begin{tabular}{lcc}
\hline & Apollo LOR ROSETTA & Apollo Direct ROSETTA \\
CM & $6,024 \mathrm{~kg}$ & $6,661 \mathrm{~kg}$ \\
SM & $24,917 \mathrm{~kg}$ & $35,972 \mathrm{~kg}$ \\
LM & $16,183 \mathrm{~kg}$ & $15,278 \mathrm{~kg}$ \\
LBM & $\mathrm{NA}$ & $74,140 \mathrm{~kg}$ \\
Stack & $47,124 \mathrm{~kg}$ & $132,051 \mathrm{~kg}$ \\
LV & $2,279,540 \mathrm{~kg}$ & $7,416,468 \mathrm{~kg}$ \\
On Pad & $2,326,664 \mathrm{~kg}$ & $7,548,519 \mathrm{~kg}$ \\
\hline
\end{tabular}


As this table shows, the Direct mission has a huge weight penalty over the LOR mission. This is mainly due to the fact that the entire three crew CM is taken to the surface and modified for lunar surface operations. The Apollo Direct has an extra staging event (LBM) and the mass is still much greater that that of the Apollo LOR. The main problem of this mission mode is the enormous mass of the launch vehicle. The $7.5 \mathrm{M} \mathrm{kg}$ gross mass far exceeds that of the Saturn V. In fact the mass of the system is the main reason that the Direct mission mode was abandoned and the Earth Orbit Rendezvous mode was created [7].

The Direct mission costs and reliabilities were not well established during the Apollo mode selection process. It was qualitatively determined that the LOR mission mode would be cheaper and faster than the Direct mission mode because of the lower system mass and the continuation of existing programs in 1965. A comparison of the Apollo Direct and Apollo LOR mission mode costs calculated from the Apollo LOR based NAFCOM CERs is given below as Table 25 and Table 26.

Table 25: Apollo LOR and DDT\&E Cost Comparison.

\begin{tabular}{lcc}
\hline$\$ M 2004$ & Apollo LOR ROSETTA & Apollo Direct ROSETTA \\
CSM & $\$ 10,773 \mathrm{M}$ & $\$ 11,555 \mathrm{M}$ \\
LM & $\$ 6,639 \mathrm{M}$ & $\$ 4,086 \mathrm{M}$ \\
LBM & $\mathrm{NA}$ & $\$ 5,206 \mathrm{M}$ \\
LV & $\$ 13,744 \mathrm{M}$ & $\$ 17,627 \mathrm{M}$ \\
Total DDTE & $\$ 31,156 \mathrm{M}$ & $\$ 38,109 \mathrm{M}$ \\
\hline
\end{tabular}


Table 26: Apollo LOR and Direct Theoretical First Unit Cost Comparison.

\begin{tabular}{lcc}
\hline$\$ M$ 2004 & Apollo LOR ROSETTA & Apollo Direct ROSETTA \\
CSM & $\$ 309 \mathrm{M}$ & $\$ 392 \mathrm{M}$ \\
LM & $\$ 733 \mathrm{M}$ & $\$ 366 \mathrm{M}$ \\
LBM & $\mathrm{NA}$ & $\$ 337 \mathrm{M}$ \\
LV & $\$ 832 \mathrm{M}$ & $\$ 1,334 \mathrm{M}$ \\
Total TFU & $\$ 1,874 \mathrm{M}$ & $\$ 2,431 \mathrm{M}$ \\
\hline
\end{tabular}

As indicated by these tables, the Apollo Direct has significantly higher costs than the Apollo LOR. This is not surprising since the Apollo Direct has significantly higher mass than the Apollo LOR using similar technologies. The Apollo Direct cost just under \$7 Billion dollars (22\%) more to develop and over $\$ 500$ million (30\%) more to produce the first unit.

A dynamically changing fault tree analysis similar to the Apollo LOR mission mode was also incorporated into the Direct ROSETTA Model. This reliability analysis was conducted for each of the ROSETTA models in the Apollo Direct and the differences between the Apollo LOR and the Apollo Direct were compared with those published in the Apollo Mission Mode Analysis [75]. The resulting reliability comparisons are given in Table 27.

Table 27: Comparison of Apollo Direct and Apollo LOR Reliability.

\begin{tabular}{lcc}
\hline & Apollo LOR ROSETTA & Apollo Direct ROSETTA \\
CSM & 0.7648 & 0.8437 \\
LM & 0.8918 & 0.9393 \\
LBM & NA & 0.9279 \\
LV & 0.7690 & 0.7228 \\
LOM & 0.5245 & 0.5315 \\
\hline
\end{tabular}


As this table shows, the resulting Apollo Direct reliability calculations are slightly higher than the Apollo LOR reliabilities. This is mainly due to the higher CSM reliability caused by the elimination of the lunar rendezvous. The LM has a significantly higher reliability for the Apollo direct because the LM is strictly a touchdown stage, without a habitat. This simpler model results in a higher reliability. The Nova reliability is slightly lower than the Saturn $\mathrm{V}$ reliability due to the larger vehicle with more engines required for the Nova launch vehicle. The total loss of mission reliability is slightly higher for the Direct mission, but the higher cost and uncertainty with the larger launch vehicle resulted in the creation of a hybrid mission mode known as Earth Orbit Rendezvous.

\subsection{EARTH ORBIT RENDEZVOUS MISSION MODE}

As a result of the high weight and cost of the Nova launch vehicle an alternative mission mode was evaluated. Werner Von Braun championed the EOR approach as a viable alternative to the favored Direct approach [7]. This mission mode was very similar to the Direct mission mode except that the launches were split between two launch vehicles. The Earth Orbit Rendezvous (EOR) involves all of the elements of the Direct mission mode with two Saturn V launch vehicles. The first Saturn V launches a tanker vehicle in place of a S-IV-B containing all of the necessary oxidizer to perform the TLI burn by the second crewed S-IV-B. The second vehicle launches the same stack as the Direct mode except the S-IV only contains enough oxidizer for the ascent. A rendezvous occurs between the tanker vehicle and the crewed stack to transfer the liquid oxygen to the S-IVB and perform the TEI burn. This mission mode is demonstrated in Figure 54. 


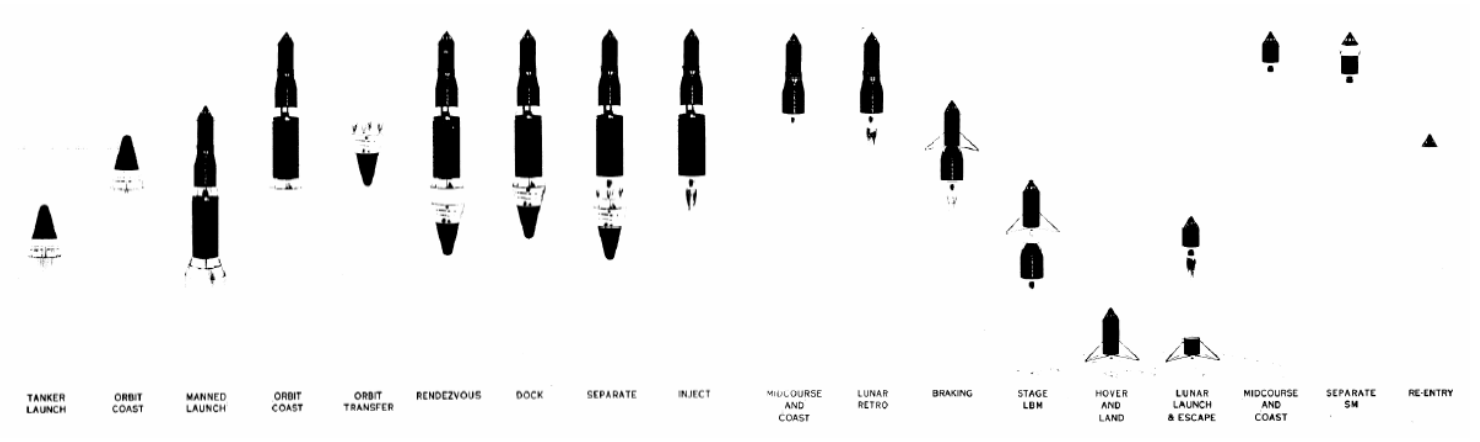

Figure 54: EOR Mission Mode [4].

As this figure shows, the EOR mission concept involves a single launch of two Saturn V launch vehicles. The Saturn V vehicles are common; and therefore, both versions of the vehicle have to be calculated for any stack weight and the larger vehicle used for both launches. A compilation of the design choices made in the Apollo EOR mission is given below as Table 28 and Table 29.

Table 28: Propellant Choices for Apollo EOR.

\begin{tabular}{lccc}
\hline Vehicle & Propellants & Engine & Engine Number \\
S-I-C & LOX/RP1 & F-1 (Gas Generator) & 5 \\
S-II & LOX/LH2 & J-2 (Gas Generator) & 5 \\
S-IVB & LOX/LH2 & J-2 (Gas Generator) & 1 \\
SM & UDMH/N204 & Pressure Fed & 1 \\
LBM & LOX/LH2 & Expander & 1 \\
LTDM & UDMH/N204 & Pressure Fed & 1 \\
\hline
\end{tabular}


Table 29: Structure Choices for Apollo EOR.

\begin{tabular}{lccc}
\hline Structure & Main Tanks & Pressurant Tanks & Other Structure \\
S-I-C & $\mathrm{Al}$ & $\mathrm{Ti}$ & $\mathrm{Al}$ \\
S-II & $\mathrm{Al}$ & $\mathrm{Ti}$ & $\mathrm{Al}$ \\
S-IVB & $\mathrm{Al}$ & $\mathrm{Ti}$ & $\mathrm{Al}$ \\
Tanker & $\mathrm{Al}$ & $\mathrm{Ti}$ & $\mathrm{Al}$ \\
CM & $\mathrm{NA}$ & $\mathrm{Al}$ & $\mathrm{Al}$ \\
SM & $\mathrm{Al}$ & $\mathrm{Ti}$ & $\mathrm{Al}$ \\
LBM & $\mathrm{Al}$ & $\mathrm{Ti}$ & $\mathrm{Al}$ \\
LTDM & $\mathrm{Al}$ & $\mathrm{Al}$ \\
\hline
\end{tabular}

As with the Apollo Direct mission mode, the EOR mission was simulated in ModelCenter using ROSETTA models and the methods described in Chapter 3. The simulation involved the use of the same ROSETTA models as Apollo Direct for the Command Module, the Service Module, the Lunar Module, LBM, and the Saturn V. These ROSETTA models were made generic enough to change the burn structure in the models so that the same model could accomplish both mission modes. The main difference is that there are two Saturn V launch vehicles. Each of these vehicle contains a calculation for both the manned and unmanned versions of the vehicle so that the larger vehicle can be used for both launches. These ROSETTA modules were then wrapped in ModelCenter to integrate the performance characteristics of the mission mode. The Architecture Cost Model (ACM) was modified to accumulate the mass, cost, and reliabilities of all of the architecture vehicles and calculate the total Life Cycle Costs (LCC) of the mission mode.

As with the Apollo Direct mission mode, the subsystem reliabilities for the EOR mission modes were derived from the Apollo Manned Lunar Landing Program Mode Comparison [75]. These reliabilities were compiled in the dynamic fault trees to 
calculate the Loss of Mission (LOM) and Loss of Crew (LOC) values for each of the ROSETTA models. A screen shot of the EOR mission mode ModelCenter tool is given as Figure 55.

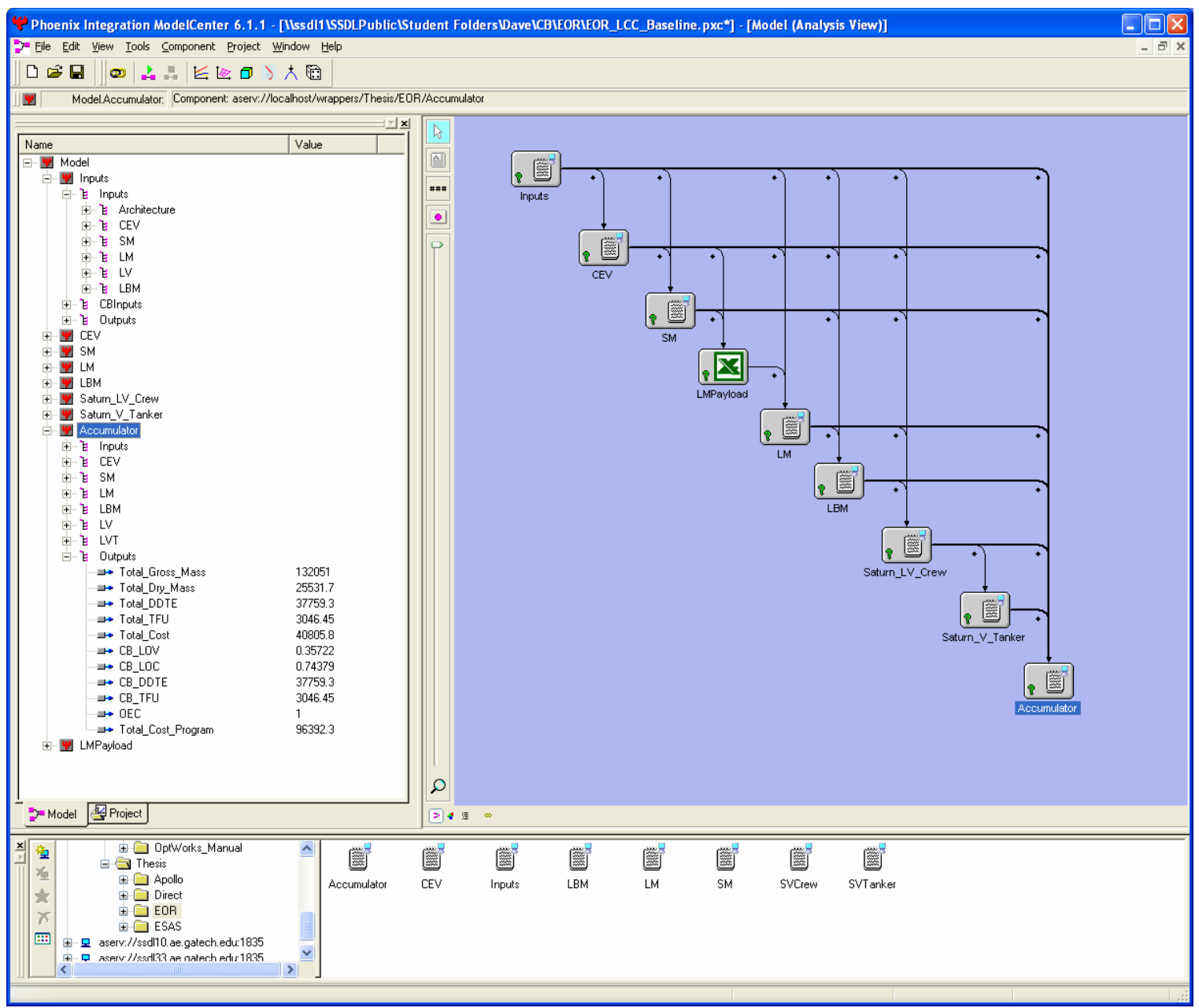

Figure 55: ModelCenter Based EOR Mission Simulation [73].

As this figure shows, the EOR mission mode simulation is very similar to the Apollo Direct mission mode. The main difference between the Apollo Direct and the Apollo EOR is the inclusion of a second Saturn V model, which has the ability to calculate both 
the manned S-IVB upper stage as well as the tanker upper stage. The Saturn V launch vehicle was also modified so that the S-II stage in the tanker provides enough delta V to travel to LEO without the assistance of a S-IVB. The Saturn V was further modified so that the S-IVB only carries enough oxidizer to perform the LEO insertion. The TLI oxidizer is then transferred by the tanker vehicle which is put into orbit by the unmanned Saturn V. Each of the Saturn V ROSETTA models calculates the masses of both variants so that the largest required stages can be used by both variants. The separate models are used only to calculate the different reliabilities and costs for the vehicles. The resulting simulation has 96 design variables (the Saturn V tanker shares the crewed inputs) and each closed architecture can be computed in less than five seconds.

The Apollo EOR baseline was computed using this ROSETTA based simulation tool. The 96 design variables were set and the ROSETTA models were evaluated to close the Apollo EOR mission mode. As with the Apollo Direct the Apollo EOR was compared with the Apollo LOR mission modes in Table 30.

Table 30: Comparison of Apollo Mission Modes.

\begin{tabular}{lccc}
\hline & Apollo LOR & Apollo Direct & Apollo EOR \\
& ROSETTA & ROSETTA & ROSETTA \\
CM & $6,024 \mathrm{~kg}$ & $6,661 \mathrm{~kg}$ & $6,661 \mathrm{~kg}$ \\
SM & $24,917 \mathrm{~kg}$ & $35,972 \mathrm{~kg}$ & $35,972 \mathrm{~kg}$ \\
LM & $16,183 \mathrm{~kg}$ & $15,278 \mathrm{~kg}$ & $15,278 \mathrm{~kg}$ \\
LBM & NA & $74,140 \mathrm{~kg}$ & $74,140 \mathrm{~kg}$ \\
Stack & $47,124 \mathrm{~kg}$ & $132,051 \mathrm{~kg}$ & $132,051 \mathrm{~kg}$ \\
LV Cargo & NA & NA & $3,781,757 \mathrm{~kg}$ \\
LV & $2,279,540 \mathrm{~kg}$ & $7,416,468 \mathrm{~kg}$ & $3,849,397 \mathrm{~kg}$ \\
On Pad & $2,326,664 \mathrm{~kg}$ & $7,548,519 \mathrm{~kg}$ & $7,763,205 \mathrm{~kg}$ \\
\hline
\end{tabular}


As this table shows, the EOR mission has the same spacecraft weight as the direct mission. The launch vehicle weight is split between the two launch vehicles. The overall mass on the pad is similar to the Direct mission, since there is a significant weight penalty associated with the separate launch vehicle (i.e. tanker vehicle).

As with the Apollo Direct mission, the Apollo EOR was compared with both the Apollo Direct and Apollo LOR mission modes. The comparison of the Apollo Direct, LOR, and Apollo EOR mission mode costs calculated from the Apollo LOR and based NAFCOM CERs is given below as Table 31 and Table 32.

Table 31: Apollo EOR, LOR and Direct Design Development Testing and Evaluation Cost Comparison.

\begin{tabular}{lccc}
\hline \$M 2004 & Apollo LOR & Apollo Direct & Apollo EOR \\
& ROSETTA & ROSETTA & ROSETTA \\
CSM & $\$ 10,773 \mathrm{M}$ & $\$ 11,555 \mathrm{M}$ & $\$ 11,555 \mathrm{M}$ \\
LM & $\$ 6,639 \mathrm{M}$ & $\$ 4,086 \mathrm{M}$ & $\$ 4,086 \mathrm{M}$ \\
LBM & $\mathrm{NA}$ & $\$ 5,206 \mathrm{M}$ & $\$ 5,206 \mathrm{M}$ \\
LV Cargo & $\mathrm{NA}$ & $\mathrm{NA}$ & $\$ 2,396 \mathrm{M}$ \\
LV & $\$ 13,744 \mathrm{M}$ & $\$ 17,627 \mathrm{M}$ & $\$ 14,880 \mathrm{M}$ \\
Total DDTE & $\$ 31,156 \mathrm{M}$ & $\$ 38,109 \mathrm{M}$ & $\$ 37,759 \mathrm{M}$ \\
\hline
\end{tabular}

Table 32: Apollo EOR, LOR, and Direct Theoretical First Unit Cost Comparison.

\begin{tabular}{lccc}
\hline \$M 2004 & Apollo LOR & Apollo Direct & Apollo EOR \\
& ROSETTA & ROSETTA & ROSETTA \\
CSM & $\$ 309 \mathrm{M}$ & $\$ 392 \mathrm{M}$ & $\$ 392 \mathrm{M}$ \\
LM & $\$ 733 \mathrm{M}$ & $\$ 366 \mathrm{M}$ & $\$ 366 \mathrm{M}$ \\
LBM & $\mathrm{NA}$ & $\$ 337 \mathrm{M}$ & $\$ 337 \mathrm{M}$ \\
LV Cargo & $\mathrm{NA}$ & $\mathrm{NA}$ & $\$ 944 \mathrm{M}$ \\
LV & $\$ 832 \mathrm{M}$ & $\$ 1,334 \mathrm{M}$ & $\$ 1,006 \mathrm{M}$ \\
Total TFU & $\$ 1,874 \mathrm{M}$ & $\$ 2,431 \mathrm{M}$ & $\$ 3,046 \mathrm{M}$ \\
\hline
\end{tabular}


As these tables show, the Apollo EOR does not offer significant cost savings over the Direct mission mode. The high cost is mainly due to the need to fly two launch vehicles per mission. The DDT\&E costs are very similar between the EOR and the Direct mission. The DDT\&E of the cargo version of the Saturn V is only $15 \%$ of the manned vehicle, since most of the components are common between the Saturn V crew and cargo and the development is already counted in the crewed vehicle. The TFU of the EOR is higher than that of the Direct mission because two Saturn V launch vehicles are $46 \%$ more costly than a single Nova vehicle. It should be noted that these costs are strictly DDT\&E and TFU. Facility costs and operations costs are not included in this analysis.

A dynamically changing fault tree analysis, similar to the Apollo Direct mission mode, was also incorporated into the EOR ROSETTA Models. This reliability analysis was conducted for each of the ROSETTA models in the Apollo EOR and the differences between the Apollo LOR and the Apollo Direct were compared with those published in the Apollo Mission Mode Analysis [75]. The resulting reliability comparisons are given in Table 33.

Table 33: Reliability Comparison of Apollo EOR, Direct, and Apollo LOR.

\begin{tabular}{lccc}
\hline & Apollo LOR & Apollo Direct & Apollo EOR \\
& ROSETTA & ROSETTA & ROSETTA \\
CSM & 0.7648 & 0.8437 & 0.8437 \\
LM & 0.8918 & 0.9393 & 0.9393 \\
LBM & NA & 0.9279 & 0.9279 \\
LV Cargo & NA & NA & 0.6653 \\
LV & 0.7690 & 0.7228 & 0.7301 \\
LOM & 0.5245 & 0.53151 & 0.3572 \\
\hline
\end{tabular}


As this table shows, the resulting Apollo EOR reliability calculations are far below that of both the Apollo LOR and the Apollo Direct. This low reliability number is mainly due to the fact that two of the lowest reliability vehicles are needed for EOR to successfully complete its mission. The spacecraft portion of the EOR architecture is exactly the same as that of the Direct missions.

\subsection{Apollo Lunar Architecture Decision}

In 1965, NASA set out with finding the ideal way of transporting humans to the lunar surface. To accomplish this goal, each of the different mission modes was compared based upon feasibility (performance), economics, and reliability. To calculate the differences each mode was evaluated independently and compared via trade studies. This decision can be reevaluated and confirmed using the models created in this thesis. From the performance results summarized in Table 30, it is obvious that the Direct mission mode requires a launch vehicle so large that it would dwarf all previous launch vehicles. This vehicle would require investments in new engine programs, facilities, and operations, which are far outside the limits of the experience in the 1960s and today. Even if these outside factors are ignored, the Direct mission is still costly for a reliability only slightly higher than that of LOR. A summary of the baseline Apollo results is given as Table 34 . 
Table 34: Summary of Apollo Lunar Architecture Decision

\begin{tabular}{lccc}
\hline & Apollo LOR & Apollo Direct & Apollo EOR \\
& ROSETTA & ROSETTA & ROSETTA \\
Total Mass & $2,326,664 \mathrm{~kg}$ & $7,548,519 \mathrm{~kg}$ & $7,763,205 \mathrm{~kg}$ \\
DDT\&E & $\$ 31,157 \mathrm{M}$ & $\$ 38,109 \mathrm{M}$ & $\$ 37,759 \mathrm{M}$ \\
TFU & $\$ 1,873 \mathrm{M}$ & $\$ 2,431 \mathrm{M}$ & $\$ 3,046 \mathrm{M}$ \\
Total Cost to First Vehicle & $\$ 33,031 \mathrm{M}$ & $\$ 40,540 \mathrm{M}$ & $\$ 40,805 \mathrm{M}$ \\
LOM & 0.5245 & 0.53151 & 0.3572 \\
LOC & 0.6900 & 0.7440 & 0.7438 \\
\hline
\end{tabular}

The LOC numbers for the different architectures are similar for the three modes. The main discrepancy between LOM and LOC for the EOR is due to the fact that the second launch has little effect on the safety of the crew. There is a small chance of LOC during the rendezvous in Earth orbit, but this risk is mitigated by the lower LOC for the smaller launch vehicle Saturn V as compared with the Nova vehicle of the Direct mission mode. From this data, LOR is obviously the lowest cost and mass between the Apollo lunar modes. This low cost and relatively high LOM reliability drove the LOR decision. This is verified in Von Braun's enumeration of why the LOR mode was chosen [7]:

1). LOR mode appeared most likely to be accomplished by the end of the decade

2). LOR has the highest performance margins (lowest mass)

3). The separation of the reentry vehicle and the lunar landing vehicle constituted the two most critical tasks in producing a lunar spacecraft and separating these two function into two separate elements was bound to simplify development of the spacecraft system....(lowest cost). 
The comments of Von Braun are verified in the analysis conducted via the ROSETTA models. The LOR has the lowest mass and cost. Reliability is not mentioned in Von Braun's comments, since reliability was treated qualitatively at this level of the Apollo decision. A separate issue that drove the LOR decision as noted in Von Braun's first comment is the Apollo program was under a strict schedule. This schedule aspect is not modeled in the ROSETTA models and is treated as an outside variable to the lunar architecture selection.

\subsection{EXPLORATION SYSTEM ARCHITECTURE STUDY BASELINE}

To verify that the methodology laid out in Chapter 3 can also be used on current lunar architecture designs the ESAS lunar architecture was simulated. The Exploration system architecture was laid out in Chapter 1. As Figure 3 shows the ESAS architecture is a combination of the Apollo EOR and LOR architectures. The ESAS architecture team set out with ambitious goals of sending 4 humans to the Moon for a total of 14 days. This far exceeds the Apollo mission goals of 3 people ( 2 to the lunar surface) for a total of 3 days on the surface. The ESAS team set out with the requirement to keep weight and costs to a minimum while designing the system to have a greater reliability than previous manned spaceflight projects. To accomplish these goals the ESAS architecture combined the LOR architecture with the EOR launch structure. Using a LOR spacecraft design allowed the spacecraft elements of the architecture to retain the low weight allowed by the leaving the command module (known as the Crew Exploration Vehicle $(\mathrm{CEV}))$ in low lunar orbit, while a dedicated lunar module travels to the surface. Unfortunately, there isn't a launch vehicle capable of reproducing the performance of the 
Saturn V launch vehicle. To solve this problem, the ESAS team split the launches of the spacecraft into two separate launches, much like the Apollo EOR architecture. The main difference between the Apollo EOR launch vehicles and the ESAS EOR is that the launch vehicles are not common. The Crew Launch Vehicle (CLV) has a much lower payload capacity than the Cargo Launch vehicle (CaLV). Because of this difference in payload capacity the CEV and the SM are launched on the CLV while the LM and Earth Departure Stage (EDS) are launched on the CaLV. The differences between Apollo and LOR architecture modes are given below as Table 35 .

Table 35: Comparison of Apollo LOR and EOR to ESAS (EOR-LOR)

\begin{tabular}{lccc}
\hline & Apollo LOR & Apollo EOR & ESAS \\
Crew to Surface & 2 & 3 & 4 \\
Lunar Surface Stay & 3 & 3 & 14 \\
Launch Vehicles & 1 (Saturn V) & 2 (Saturn V) & 2 (CLV \& CaLV) \\
TLI Burn & S-IVB & S-IVB & EDS (CaLV) \\
LOI Burn & SM & SM & LM \\
Decent to Lunar Surface & LM & LBM & LM \\
Ascent from Lunar Surface & LM & SM & LM \\
TEI & SM & SM & SM \\
Entry Descent and Landing & CM & CM & CEV (CM) \\
\hline
\end{tabular}

As this table shows, there is also a difference between the Apollo LOR and ESAS LOR burn structures in that the LM performs the breaking maneuver into LLO in the ESAS architecture, while the SM performs the breaking maneuver in the Apollo LOR architecture. This difference is driven by the fact that the SM gross mass is limited by the payload capacity of the CLV. To keep the SM gross mass down, the LOI insertion burn is performed by the LM and not the SM. 
The ESAS architecture team completed a series of trade studies to attempt to find the optimal configuration choices for the EOR-LOR architecture. A compilation of the design choices made in the ESAS architecture is given below as Table 36 and Table 37.

Table 36: Propellant Choices for ESAS.

\begin{tabular}{lccc}
\hline Vehicle & Propellants & Engine & Engine Number \\
CLV 1 $1^{\text {st }}$ Stage & HTPB & 4-Segment RSRM & 1 \\
CLV 2 Stage & LOX/LH2 & SSME (Staged Comb) & 1 \\
CaLV Booster & HTPB & 5-Segment RSRM & 2 \\
CaLV 1 ${ }^{\text {st }}$ Stage & LOX/LH2 & SSME (Staged Comb) & 5 \\
CaLV 2 ${ }^{\text {nd }}$ Stage & LOX/LH2 & J-2 (Gas Generator) & 2 \\
SM & LOX/CH4 & Pressure Fed & 1 \\
LM Descent & LOX/LH2 & Rl-10 Expander & 1 \\
LM Ascent & LOX/CH4 & Pressure Fed & 1 \\
\hline
\end{tabular}

Table 37: Structure Choices for ESAS.

\begin{tabular}{lccc}
\hline Structure & Main Tanks & Pressurant Tanks & Other Structure \\
CLV 2 ${ }^{\text {nd }}$ Stage & Al-Li & Gr-Ep & Al-Li \\
CaLV 1 $1^{\text {st }}$ Stage & Al-Li & Gr-Ep & Al-Li \\
CaLV 2 ${ }^{\text {nd }}$ Stage & Al-Li & Gr-Ep & Al-Li \\
CEV & NA & Gr-Ep & Al \\
SM & Gr-Ep & Gr-Ep & Al \\
LM Descent & Gr-Ep & Gr-Ep & Al-Li \\
LM Ascent & Gr-Ep & Gr-Ep & Al-Li \\
\hline
\end{tabular}

Graphite Epoxy wrapped tank are prevalent in the ESAS mission mode. This is a new technology that was not available in the Apollo era and is therefore only available in the ESAS architecture. The ESAS architecture also takes advantage of existing engine programs. The SSME and 4-segment solid rocket boosters are both products of the 
shuttle program and are therefore only a minimal amount of DDT\&E (air-start SSME, control for SRB, etc.). The J-2 engine also has a reduction in DDT\&E cost due to the fact that it is a derivative of an Apollo style engine that was already been proven. The RL-10 used on the LM descent engine also has spacecraft heritage (Atlas and Delta launch vehicles) and would only require minimal DDT\&E to be used as a lunar descent engine (deep throttleability). These engine cost reductions allow the EOR-LOR mission mode with seven different engines to be viable.

The EOR-LOR mission mode was simulated in ModelCenter using ROSETTA models and the methods described in Chapter 3. The simulation involved the use of the Apollo era ROSETTA models for the Command Module, the Service Module, the Lunar Module, as well as the creation of the CLV and CaLV models. The Apollo era models required modifications in subsystem reliabilities and engine reliabilities so that the resulting LOM and LOC numbers were correct for 2007 technologies. These ROSETTA modules were then wrapped in ModelCenter to integrate the performance characteristics of the mission mode. The Architecture Cost Model (ACM) was also integrated to compile the costs of the different vehicles to then calculate the total Life Cycle Costs (LCC) of the mission mode.

As with the Apollo modes, each of the different ROSETTA models were constructed using Response Surface Methods for performance calculations, NAFCOM based cost estimations, and dynamically changing fault trees for the reliability analysis. As noted above, the subsystem reliabilities (ECLSS, Avionics, Guidance and Navigation) were modified so that the baseline numbers were in line with current 2007 technologies. These reliabilities were compiled in the dynamic fault trees to calculate the 
LOM and LOC values for each of the ROSETTA models. A screen shot of the ModelCenter tool constructed for the ESAS mission mode is given as Figure 56.

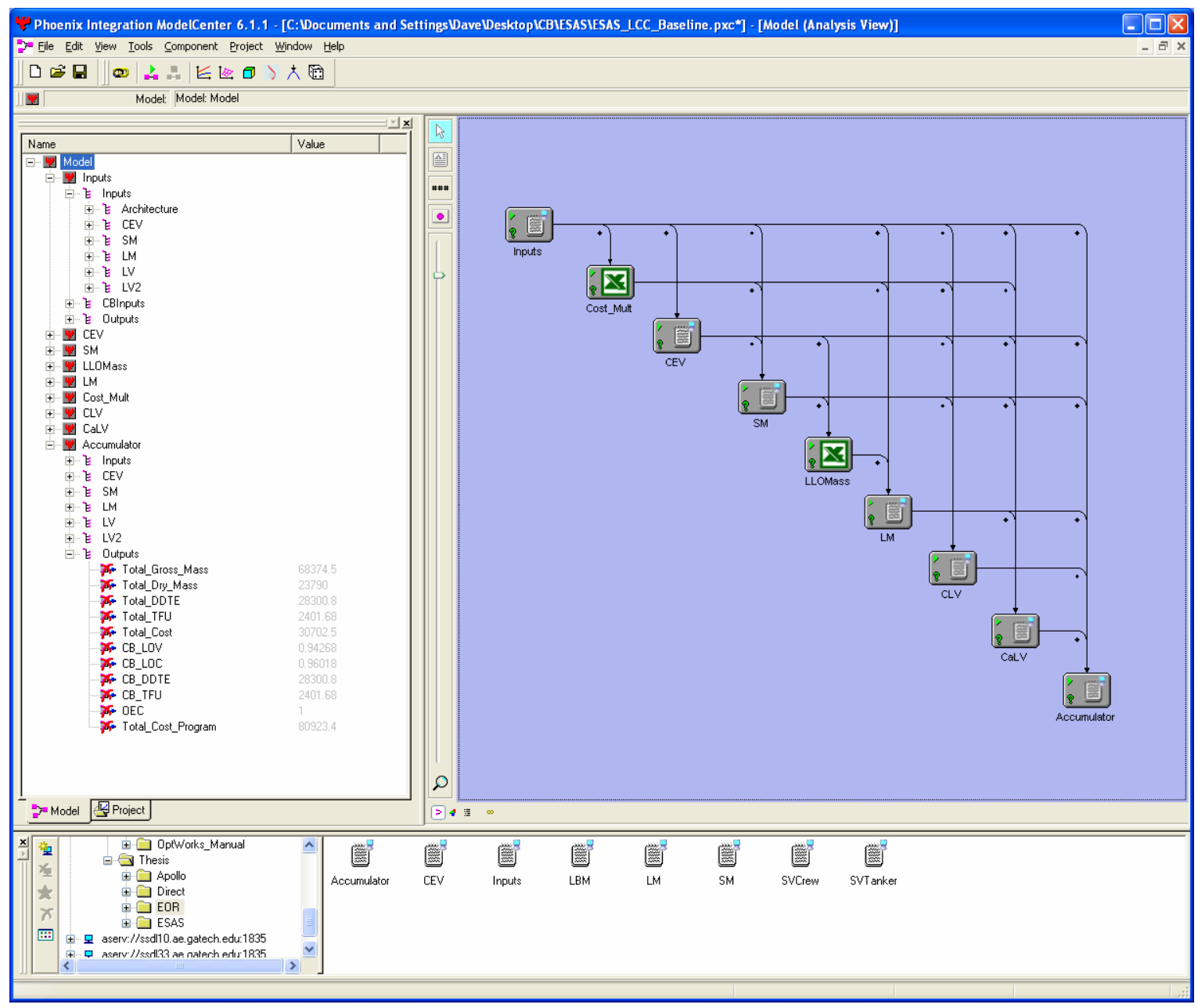

Figure 56: ModelCenter Based ESAS Simulation [73].

As with the Apollo mission modes, the ESAS mission mode was treated as a DSM in ModelCenter. The main difference between the ESAS architecture simulation and the Apollo simulation is the order of the contributing analyses. In the ESAS simulation, the SM must be calculated before the LM. This is because the LM must perform the LOI 
burn with the SM and CM (CEV) as payload. This LOR breaking performed by the LM is unique for the ESAS architecture.

The ESAS baseline was computed using this ROSETTA based simulation tool. The 86 design variables were set and the ROSETTA models were evaluated to close the ESAS mission mode. The performance results were compared between the simulation results and the actual ESAS vehicle weights as given in the ESAS final report [13]. The results are given as Table 38 .

Table 38: Comparison of Apollo LOR Performance Results.

\begin{tabular}{lccc}
\hline & ESAS (Report) & ROSETTA Models & $\%$ Diff \\
CEV & $9,506 \mathrm{~kg}$ & $9,526 \mathrm{~kg}$ & $0.21 \%$ \\
SM & $13,647 \mathrm{~kg}$ & $13,668 \mathrm{~kg}$ & $0.15 \%$ \\
LM & $45,864 \mathrm{~kg}$ & $45,180 \mathrm{~kg}$ & $-1.49 \%$ \\
Stack & $69,017 \mathrm{~kg}$ & $68,374 \mathrm{~kg}$ & $-0.93 \%$ \\
CLV & $805,309 \mathrm{~kg}$ & $803,473 \mathrm{~kg}$ & $-0.23 \%$ \\
CaLV & $2,900,288 \mathrm{~kg}$ & $2,861,257 \mathrm{~kg}$ & $-1.35 \%$ \\
Total & $3,774,614 \mathrm{~kg}$ & $3,733,104 \mathrm{~kg}$ & $-1.10 \%$ \\
\hline
\end{tabular}

As this table shows, the mass results for the ROSETTA models are within $2 \%$ of the vehicle weights as they are calculated in the ESAS final report. The biggest discrepancy is on the LM. This discrepancy is only $300 \mathrm{~kg}$ over a gross mass of over $45,000 \mathrm{~kg}$.

The mass estimates for the ESAS mission mode are very accurate and therefore a mass-based cost comparison can therefore be completed. Unfortunately, the calculated ESAS costs are not publicly available and therefore the ESAS costs can only be compared with the Apollo mission mode costs demonstrated in Table 39. 
Table 39: ESAS Design Development Testing and Evaluation Cost Comparison.

\begin{tabular}{lccc}
\hline$\$ M 2004$ & $E S A S$ & Apollo $L O R$ & Apollo EOR \\
CEV/SM & $\$ 10,763 \mathrm{M}$ & $\$ 10,505 \mathrm{M}$ & $\$ 11,555 \mathrm{M}$ \\
$\mathrm{LM}$ & $\$ 7,013 \mathrm{M}$ & $\$ 6,752 \mathrm{M}$ & $\$ 4,086 \mathrm{M}$ \\
$\mathrm{LBM}$ & $\mathrm{NA}$ & $\mathrm{NA}$ & $\$ 5,206 \mathrm{M}$ \\
Crew LV & $\$ 4,990 \mathrm{M}$ & $\$ 13,887 \mathrm{M}$ & $\$ 2,396 \mathrm{M}$ \\
Cargo LV & $\$ 9,174 \mathrm{M}$ & $\mathrm{NA}$ & $\$ 14,880 \mathrm{M}$ \\
Total & $\$ 32,663 \mathrm{M}$ & $\$ 31,144 \mathrm{M}$ & $\$ 37,759 \mathrm{M}$ \\
\hline
\end{tabular}

Table 40: ESAS Theoretical First Unit Cost Comparison.

\begin{tabular}{lccc}
\hline$\$ M 2004$ & $E S A S$ & Apollo LOR & Apollo EOR \\
CEV/SM & $\$ 528 \mathrm{M}$ & $\$ 294 \mathrm{M}$ & $\$ 392 \mathrm{M}$ \\
$\mathrm{LM}$ & $\$ 990 \mathrm{M}$ & $\$ 651 \mathrm{M}$ & $\$ 366 \mathrm{M}$ \\
$\mathrm{LBM}$ & $\mathrm{NA}$ & $\mathrm{NA}$ & $\$ 337 \mathrm{M}$ \\
Crew LV & $\$ 161 \mathrm{M}$ & $\$ 901 \mathrm{M}$ & $\$ 944 \mathrm{M}$ \\
Cargo LV & $\$ 719 \mathrm{M}$ & $\mathrm{NA}$ & $\$ 1,006 \mathrm{M}$ \\
Total TFU & $\$ 4,388 \mathrm{M}$ & $\$ 1,846 \mathrm{M}$ & $\$ 3,046 \mathrm{M}$ \\
\hline
\end{tabular}

As these tables show, the DDT\&E for the ESAS structure is simulated to be slightly more than that of the Apollo LOR, but less than the Apollo EOR. This is a surprising result since the ESAS architecture is billed as a low cost architecture. The high DDT\&E can be attributed to the size of the vehicles necessary to take 4 crew to the surface for 14 days with almost twice the volume per crew member as Apollo. That is twice the crew of the Apollo LOR and one and a half times the crew of the Apollo EOR. The surface stay time is almost five times the surface stay time of both Apollo missions. So taking these facts into account the ESAS architecture is actually an inexpensive architecture when compared to Apollo. This can be demonstrated as a cost per man hour on the moon. 
Table 41: Cost per Man Hour on the Moon

\begin{tabular}{lcc}
\hline & $E S A S$ & Apollo LOR \\
DDTE & $\$ 32,663 \mathrm{M}$ & $\$ 31,144 \mathrm{M}$ \\
TFU & $\$ 4,388 \mathrm{M}$ & $\$ 1,846 \mathrm{M}$ \\
Total $(\$ M)$ & $\$ 37,051 \mathrm{M}$ & $\$ 32,990 \mathrm{M}$ \\
People on Surface & 4 & 2 \\
Days on Surface & 14 & 3 \\
Man Hours on Moon & 1,344 & 144 \\
Cost per Man-Hour $(\$ \mathrm{M})$ & $\$ 28 \mathrm{M}$ & $\$ 229 \mathrm{M}$ \\
\hline
\end{tabular}

As this table shows the ESAS cost per man-hour is an order of magnitude less than the Apollo LOR architecture. As with the Apollo EOR, ESAS requires the development of two separate launch vehicles. These vehicles account for $43 \%$ of the total DDT\&E of the ESAS architecture. It should also be noted that the launch vehicles have heritage engines. These heritage engines are given a cost reduction to account for the decreased cost.

The dynamically changing fault tree analysis incorporated in each ROSETTA model calculates the total vehicle reliability based upon the assumed subsystem reliabilities and the vehicle configurations. This reliability analysis was conducted for each of the ROSETTA models in the ESAS mission mode and the results were compared with those published in the ESAS final report [13]. The resulting reliability comparisons are given in Table 42 . 
Table 42: Apollo LOR Loss of Mission Reliability Comparison.

\begin{tabular}{lccc}
\hline & ESAS (Report) & ROSETTA Models & $\%$ Diff \\
CEV & NA & 0.9957 & NA \\
SM & NA & 0.9884 & NA \\
LSAM & NA & 0.9719 & NA \\
CLV & 0.9976 & 0.9972 & $-0.04 \%$ \\
CaLV & 0.9911 & 0.9883 & $-0.28 \%$ \\
Total & 0.9380 & 0.9427 & $0.50 \%$ \\
\hline
\end{tabular}

As this table shows, the ROSETTA model LOM reliability calculations via the dynamically changing fault trees are very close to the calculated reliability from the ESAS report. The CEV, SM, and LSAM reliabilities are not publicly available, but the overall LOM reliabilities for the mission are publicly available via the ESAS final report. The overall calculated mission reliability is within $1 \%$ of the published ESAS numbers. The ESAS reliabilities are much higher than the Apollo LOM numbers due to the increased subsystem reliability through technologies that have matured between the 1960s and the today. ECLSS, Avionics, and Guidance systems have all matured throughout the Space Shuttle and International Space Station programs. Because of this maturity, the subsystem failure rate is assumed to be $10 \%$ of that of the Apollo systems. The engine reliabilities are assumed to be the same as that of the Apollo era.

The calculated ESAS reliabilities result in a LOM probability of 0.9427 or one loss of mission in every 17.5 missions. This reliability is far superior to the Apollo Era calculated and demonstrated reliabilities. This increased reliability is a result of the higher subsystem reliabilities as well as the improved launch vehicle reliabilities. The CLV has a calculated failure rate of 1 in 357 flights and the CaLV has a calculated failure rate of 1 in 85 flights. These vehicles have a combined launch vehicle reliability of 
0.9855 , or a failure rate of 1 in 69 missions. This reliability is far higher than the calculated Saturn V reliability of 0.7690 , or a failure rate of 1 in every 4 missions. This increased reliability is a function of the experience gained in the Space Shuttle program which matured most of the subsystems (engines, avionics, and structures) in use in the ESAS launch vehicles.

\subsection{COMPARISON OF EXPLORATION SYSTEM ARCHITECTURE STUDY MisSION Mode AND APOLlo LOR Mission ModE}

The ESAS architecture is a program which is touted as a mission mode that has greater capacities than Apollo at a much lower cost. As tables Table 39 and Table 40 show the DDT\&E and TFU of ESAS is comparable to that of the Apollo LOR mission mode. This is attributed to the higher capacity of the ESAS mission (4 crew, twice the volume per crew, 14 days on the surface) over the Apollo Missions. This section compares the Apollo LOR mission mode with the ESAS LOR-EOR mission mode for a 3

crew, 3 day lunar surface stay with a volume per crew of the Apollo era $3.315 \mathrm{~m}^{3}$. This comparison keeps the configurations choices (propellants, engine types, etc.) for the different mission modes, but compares the modes with the same architecture requirements. It should be noted that even with the architecture requirements change for the ESAS (Apollo) mission, the performance requirements are not completely the same since the ESAS architecture has the ability to reach any point on the moon whereas the Apollo architectures are restricted to an equatorial landing site. A comparison of the ESAS Report, Apollo LOR, and ESAS architecture with the Apollo mission parameters is included as Table 43 . 
Table 43: Comparison of ESAS and Apollo LOR Performance Results for a Three Crew

Mission.

\begin{tabular}{lccc}
\hline \$M 2004 & ESAS Report & ESAS (Apollo) & Apollo LOR \\
CEV & $9,526 \mathrm{~kg}$ & $5,184 \mathrm{~kg}$ & $6,024 \mathrm{~kg}$ \\
SM & $13,668 \mathrm{~kg}$ & $8,680 \mathrm{~kg}$ & $24,917 \mathrm{~kg}$ \\
LM & $45,180 \mathrm{~kg}$ & $35,854 \mathrm{~kg}$ & $16,183 \mathrm{~kg}$ \\
Spacecraft Total & $68,374 \mathrm{~kg}$ & $49,718 \mathrm{~kg}$ & $47,124 \mathrm{~kg}$ \\
Crewed Launch Vehicle & $803,473 \mathrm{~kg}$ & $725,818 \mathrm{~kg}$ & $2,279,540 \mathrm{~kg}$ \\
Cargo Launch Vehicle & $2,861,257 \mathrm{~kg}$ & $2,560,347 \mathrm{~kg}$ & NA \\
Total & $3,733,104 \mathrm{~kg}$ & $3,335,883 \mathrm{~kg}$ & $2,326,664 \mathrm{~kg}$ \\
\hline
\end{tabular}

As this table shows, the ESAS (Apollo) spacecraft weights are comparable with the Apollo LOR weights. The CEV weight is significantly lower due to the higher technology of the avionics and ECLSS systems over the Apollo systems. The SM weight is significantly lower for the SM because of the elimination of the LOI burn from the ESAS vehicle. The higher costs of the ESAS architecture could also be attributed to a conservative percentage (10\%) put on the DDT\&E of the heritage engines used in ESAS. The ESAS service module also has advantage of the use of higher performance propellants (LOX/CH4 vs. UDMH/N2O4). The LM is significantly heavier for the ESAS mission mode because of the addition of the LOI burn to the LM. This increased Delta V adds to the propellant requirements for the LM. The spacecraft stack weights are slightly higher for the ESAS (Apollo) since the ESAS mode requires increased hardware to break the spacecraft into two separate launches. This separation of launches, as well as the performance requirement to reach any point on the Moon, results in a slightly higher spacecraft weight. 
Even with the slightly higher weights of the ESAS (Apollo) architecture over the Apollo LOR, the resulting DDT\&E costs are significantly lower. This lower DDT\&E is due to the fact that the ESAS architectures use existing engines for most of the spacecraft and launch vehicles (SSME, RL-10, and SRBs). The decreased engine costs result in a significantly lower DDT\&E for the system. A summary of the DDT\&E costs for the different architectures is given as Table 44 .

Table 44: Comparison of ESAS and Apollo LOR Design, Development, Testing and Evaluation Results for a Three Crew Mission.

\begin{tabular}{lccc}
\hline$\$ M 2004$ & ESAS Report & ESAS (Apollo) & Apollo LOR \\
CEV & $\$ 5,356 \mathrm{M}$ & $\$ 4,306 \mathrm{M}$ & $\$ 6,258 \mathrm{M}$ \\
SM & $\$ 5,407 \mathrm{M}$ & $\$ 4,386 \mathrm{M}$ & $\$ 4,513 \mathrm{M}$ \\
LM & $\$ 7,014 \mathrm{M}$ & $\$ 6,259 \mathrm{M}$ & $\$ 6,639 \mathrm{M}$ \\
Crewed Launch Vehicle & $\$ 4,990 \mathrm{M}$ & $\$ 4,353 \mathrm{M}$ & $\$ 13,476 \mathrm{M}$ \\
Cargo Launch Vehicle & $\$ 9,174 \mathrm{M}$ & $\$ 8,411 \mathrm{M}$ & $\mathrm{NA}$ \\
Total & $\$ 31,941 \mathrm{M}$ & $\$ 27,714 \mathrm{M}$ & $\$ 31,158 \mathrm{M}$ \\
\hline
\end{tabular}

The Theoretical First Unit costs for ESAS (Apollo) architectures are similar to the Apollo LOR architecture. The ESAS (Apollo) TFU costs are higher because of the high costs of the pump fed engines used for the ESAS (Apollo). The Apollo LOR uses cheaper pressure fed engines. The ESAS (Apollo) also has more components (two launch vehicles) which required different manufacturing lines and usually result in higher costs. The comparison of the TFU for the ESAS and Apollo LOR architecture is given as Table 45. 
Table 45: Comparison of ESAS and Apollo LOR Theoretical First Unit Results for a Three Crew Mission.

\begin{tabular}{lccc}
\hline$\$ M 2004$ & ESAS Report & ESAS (Apollo) & Apollo LOR \\
CEV & $\$ 191 \mathrm{M}$ & $\$ 151 \mathrm{M}$ & $\$ 140 \mathrm{M}$ \\
SM & $\$ 337 \mathrm{M}$ & $\$ 260 \mathrm{M}$ & $\$ 169 \mathrm{M}$ \\
LM & $\$ 990 \mathrm{M}$ & $\$ 859 \mathrm{M}$ & $\$ 733 \mathrm{M}$ \\
Crewed Launch Vehicle & $\$ 162 \mathrm{M}$ & $\$ 141 \mathrm{M}$ & $\$ 832 \mathrm{M}$ \\
Cargo Launch Vehicle & $\$ 719 \mathrm{M}$ & $\$ 645 \mathrm{M}$ & $\mathrm{NA}$ \\
Total & $\$ 2,399 \mathrm{M}$ & $\$ 2,056 \mathrm{M}$ & $\$ 1,874 \mathrm{M}$ \\
\hline
\end{tabular}

When the ESAS (Apollo) and the Apollo LOR architectures are compared to the ESAS (Apollo) architectures, the ESAS vehicles have a slightly higher weight due to the addition of a second launch vehicle and the requirement to land at any point on the surface of the Moon. The DDT\&E costs for the ESAS (Apollo) vehicles are lower than those of the Apollo LOR vehicles because of the use of existing engines to lower the engine DDT\&E costs. The TFU costs are higher for the ESAS (Apollo) architecture because of the use of complex pump fed engines over the cheaper and simpler pressure fed engines.

\subsection{VALIDATION CONCLUSIONS}

The lunar architecture selection tool created in this research provides accurate answers for the performance, cost, and reliability of the lunar architectures simulated. These lunar architectures included both historical and current designs with varying mission modes. When actual costs were available, the performance, cost, and reliability calculations were all within $10 \%$ for all of the simulated architectures. The mass of the architectures was particularly accurate, falling within $2 \%$ for all mission modes. When 
the comparison data was not available for an architecture mode it was baselined against the other modes calculated and differences in mass, cost, and reliability were scrutinized. As expected, the EOR and Direct spacecraft were the heaviest of the Apollo modes. The EOR mission provided the lowest reliability due to the two launch vehicles, but the loss of crew more comparable with the other modes since the second launch has little effect on the safety of the crew. The Direct mission LOR had a similar loss of crew number as the EOR, but the single larger launch vehicle actually resulted in a lower life cycle cost than the two smaller vehicles. This result was surprising. The DDT\&E was higher for the Direct mission, but the cost of two launch vehicles per mission pushed the LCC cost of the EOR higher than the LCC of the Direct mission.

The ESAS mission was also validated against the ESAS final report findings. The ESAS ROSETTA simulation was found to be within $2 \%$ of the total spacecraft mass. The reliability findings were also within $1 \%$ for the entire mission. The cost numbers were not available for comparison, so the ESAS mode was compared with the Apollo cost numbers. It was found that the ESAS mode was more expensive than expected for the baseline missions (approximately that of Apollo). This high cost was a result of the greater mission capabilities of the ESAS mission over the Apollo. To compare apples to apples, the ESAS mission was resimulated with the Apollo mission requirements. This comparison resulted in a lower DDT\&E cost for ESAS mission as expected. The TFU cost was still significantly higher for the ESAS mission because of the two launch vehicles necessary to complete the mission. 


\section{CHAPTER 5}

\section{APPLICATION OF LUNAR ARCHITECTURE METHODOLOGY FOR DIFFERENT LUNAR MODES}

Chapter 3 described the method that is used to supply the decision maker with the ability to choose different optimized lunar architectures based upon the high level design discriminators. This tool was then created in an integrated environment with a combination of ROSETTA models. Chapter 4 provided a validation of this tool against the historical Apollo lunar architecture modes and the current design methodology of the ESAS team. This chapter applies the lunar architecture tool to the various mission modes described in Chapter 4 to create the ideal Pareto frontiers. These Pareto frontiers contain the idea architecture element configurations for the mission modes, which will be used by the decision maker to find the ideal reliability for a given architecture cost.

\subsection{Creation of Pareto Frontiers for Different Lunar Mission} MODES

In Chapter 4, the methodology of this thesis was baselined against the existing lunar architectures. Now that the ROSETTA models and the ModelCenter tool has been successfully verified against existing vehicles and design techniques, the methodology outlined in Chapter 3 will be implemented to find the ideal Pareto frontier for each of the 
mission modes under consideration. To create the ideal Pareto frontiers of the reliability and costs of each of the lunar architectures, a genetic algorithm was added to the models verified in Chapter 4 to find the optimal vehicle configurations for a specific weighting on the Overall Evaluation Criteria (OEC). The weightings on the OEC was then changed systematically from a $100 \%$ cost-centric to $100 \%$ reliability-centric design and then optimized at each weighting point. The combination of these points creates the ideal Pareto frontier for the mission mode.

The GA was configured to control the design variables for each of the vehicles in the lunar mode. The objective function is the OEC comprised of LOM reliability and Life Cycle Cost (LCC) which was given as equation 27. A GA was chosen as the optimization scheme of choice due to the high percentage of discrete variables (propellant type, structure type, engine cycle type, etc.). The GA was set up to have a population size of 200 with 15 participants in the tournament portion of the GA. The crossover rate probability was set at $70 \%$ with a mutation rate of $10 \%$. The GA was required to continue iterating until 10 generations are completing without improving the OEC.

To compare the different Pareto frontiers, generic lunar mission requirements were created and compiled in a lunar campaign. Each mission mode was compared on for a 20 year lunar campaign. This campaign involved a spread of the DDT\&E over the first 9 years of the campaign. After this initial development phase, a flight rate of 2 missions per year was assumed for the remaining 16 years of the campaign. The resulting costs for the 32 flights and the DDT\&E were compiled to create a total Life Cycle Cost (LCC) of the campaign. This LCC was then compiled into the OEC, via an Excel worksheet, to be optimized by the GA. 
The lunar architecture selection tool requires top level performance requirements to evaluate the lunar architectures. To compare each of the ideal Pareto frontiers to the baseline Apollo modes each of the Apollo architectures simulated had the same Apollo top level performance requirements. A summary of those requirements for each of the Apollo modes is given as Table 46.

Table 46: Architecture Inputs for the Apollo Lunar Architecture modes

\begin{tabular}{lccc}
\hline & LOR & DIR & $E O R$ \\
Total Crew & 3 & 3 & 3 \\
Crew to Surface & 2 & 3 & 3 \\
Number of Lunar Flights/Yr & 2 & 2 & 2 \\
Number of Program Yrs & 20 & 20 & 20 \\
Time on Lunar Surface & 3 & 3 & 3 \\
Number of Days SM Provides Power & 9 & 3 & 3 \\
Number of Days LM Provides Power & 3 & 9 & 9 \\
Crew Escape System & $\mathrm{Y}$ & $\mathrm{Y}$ & $\mathrm{Y}$ \\
CM Volume/Crew & 4.69 & 4.69 & 4.69 \\
Commonality w/SM and LM Engines & $\mathrm{Y}$ & $\mathrm{Y}$ & $\mathrm{Y}$ \\
\hline
\end{tabular}

Once the top level constraints are defined, there are a number of vehicle level design variables that can be changed by the lunar architecture selection tool. These variables change the performance, cost, and reliability of each of the vehicle elements. These vehicle changes then cascade throughout the entire architecture and affect the performance, cost, and reliability of all of the other vehicle elements of the system. A morphological matrix of the design variables that can be changed for each of the elements in a lunar architecture is included as Table 47 for the CM, Table 48 for the LM, Table 49 for the SM, and Table 50 for the launch vehicle. 
Table 47: Morphological Matrix for Command Module ROSETTA Model

\begin{tabular}{cccccc}
\hline Structure Type & $\mathrm{Al}$ & $\mathrm{Al}-\mathrm{Li}$ & $\mathrm{Ti}$ & $\mathrm{Gr}-\mathrm{Ep}$ & $\mathrm{MMC}$ \\
Battery Power & $0 \mathrm{~kW}-\mathrm{hr}$ & $150 \mathrm{~kW}-\mathrm{hr}$ & & & \\
Battery SED & $0 \mathrm{~kW}-\mathrm{hr} / \mathrm{kg}$ & $0.25 \mathrm{~kW}-\mathrm{hr} / \mathrm{kg}$ & & \\
RCS Type & LOX/LH2 & LOX/CH4 & UDMH/N2O4 & \\
Avionics Redundancy & 0 & 1 & & \\
ECLSS Redundancy & 0 & 1 & \\
Power Redundancy & 0 & 1 & \\
Parachute Redundancy & 0 & 1 & & \\
\hline
\end{tabular}

Table 48: Morphological Matrix for Lunar Module ROSETTA Model

\begin{tabular}{lcclll}
\hline Payload to LS & 0 & 50000 & & & \\
Payload to LLO & 0 & 10000 & & & \\
Number of Stages & 1 & 2 & & \\
Ascent Fuel Type & LOX/LH2 & LOX/CH4 & UDMH/N2O4 & \\
Fuel Type & LOX/LH2 & LOX/CH4 & UDMH/N2O4 & \\
Fuel Cell Power & $0 \mathrm{~kW}$ & $100 \mathrm{~kW}$ & & & \\
Battery Power & $0 \mathrm{~kW}-\mathrm{hr}$ & $150 \mathrm{~kW}-\mathrm{hr}$ & & & \\
Battery SED & $0 \mathrm{~kW}-\mathrm{hr} / \mathrm{kg}$ & $0.25 \mathrm{~kW}-\mathrm{hr} / \mathrm{kg}$ & & & \\
T/W @ LS & 1.80 & 3 & & & \\
T/W @ LLO Descent & 1.80 & 5 & & & \\
EO on Ascent Stage & 0 & 1 & & & \\
EO on Descent Stage & 0 & 1 & & & \\
\# Ascent Engines & 1 & 5 & & & \\
\# Descent Engines & 1 & 5 & & & \\
Area Ratio Ascent & 50 & 150 & & & \\
Area Ratio Descent & 50 & 150 & & & \\
Ascent Cycle Type & Expander & Pressure & & & \\
Descent Cycle Type & Expander & Pressure & & & \\
Ascent Tanks & $\mathrm{Al}$ & $\mathrm{Al}-\mathrm{Li}$ & $\mathrm{Ti}$ & Gr-Ep & MMC \\
Descent Tanks & $\mathrm{Al}$ & $\mathrm{Al}-\mathrm{Li}$ & $\mathrm{Ti}$ & Gr-Ep & MMC \\
Crew Comp. Material & $\mathrm{Al}$ & $\mathrm{Al}-\mathrm{Li}$ & $\mathrm{Ti}$ & Gr-Ep & MMC \\
He Tank Material & $\mathrm{Al}$ & $\mathrm{Al}-\mathrm{Li}$ & $\mathrm{Ti}$ & Gr-Ep & MMC \\
Structure Material & $\mathrm{Al}$ & Al-Li & Ti & Gr-Ep & MMC \\
\hline
\end{tabular}


Table 49: Morphological Matrix for Service Module ROSETTA Model

\begin{tabular}{lccccc}
\hline Propellant Type & LOX/LH2 & LOX/CH4 & UDMH/N2O4 & & \\
Engine Type & Expander & Pressure & & & \\
Structural Material & $\mathrm{Al}$ & $\mathrm{Al}-\mathrm{Li}$ & $\mathrm{Ti}$ & $\mathrm{Gr}-\mathrm{Ep}$ & $\mathrm{MMC}$ \\
Engine Redundancy & 0 & 1 & & & \\
Power Redundancy & 0 & 1 & & & \\
TPS Type & $\mathrm{MLI}$ & $\mathrm{Cryo-cooler}$ & & & \\
Pressurant Material & $\mathrm{Al}$ & $\mathrm{Al}-\mathrm{Li}$ & $\mathrm{Ti}$ & $\mathrm{Gr}-\mathrm{Ep}$ & $\mathrm{MMC}$ \\
RCS Type & $\mathrm{LOX} / \mathrm{LH} 2$ & $\mathrm{LOX} / \mathrm{CH} 4$ & $\mathrm{UDMH} / \mathrm{N} 2 \mathrm{O} 4$ & & \\
Number of Engines & 1 & 5 & & & \\
\hline
\end{tabular}

Table 50: Morphological Matrix for Launch Vehicle ROSETTA Model

\begin{tabular}{lcclll}
\hline Stage 1 Engine Type & Gas Generator & Staged-Combustion & & & \\
Stage 1 Propellant Type & LOX/LH2 & LOX/RP1 & & & \\
Stage 1 Structure Type & $\mathrm{Al}$ & $\mathrm{Al}-\mathrm{Li}$ & $\mathrm{Ti}$ & $\mathrm{Gr}-\mathrm{Ep}$ & $\mathrm{MMC}$ \\
Stage 1 \# Engines & 1 & 10 & & & \\
Stage 1 T/W & 1.2 & 2 & & & \\
Stage 2 Engine Type & Gas Generator & Staged-Combustion & & & \\
Stage 2 Propellant Type & LOX/LH2 & LOX/RP1 & & & \\
Stage 2 Structure Type & $\mathrm{Al}$ & $\mathrm{Al}-\mathrm{Li}$ & $\mathrm{Ti}$ & $\mathrm{Gr}-\mathrm{Ep}$ & $\mathrm{MMC}$ \\
Stage 2 \# Engines & 1 & 10 & & & \\
Stage 2 T/W & 0.5 & 2 & & & \\
Stage 3 Engine Type & Gas Generator & Staged-Combustion & & & \\
Stage 3 Propellant Type & LOX/LH2 & LOX/RP1 & & & \\
Stage 3 Structure Type & $\mathrm{Al}$ & $\mathrm{Al}-\mathrm{Li}$ & $\mathrm{Ti}$ & $\mathrm{Gr}-\mathrm{Ep}$ & $\mathrm{MMC}$ \\
Stage 3 \# Engines & 1 & 10 & & & \\
Stage 3 T/W & 0.5 & 2 & & & \\
Crew Escape System & $\mathrm{Y}$ & $\mathrm{N}$ & & & \\
EO 1st Stage & 0 & 1 & & & \\
EO 2nd Stage & 0 & 1 & & & \\
EO 3rd Stage & 0 & 1 & & & \\
\hline
\end{tabular}


These morphological matrices give a subset of the total amount of inputs that the lunar architecture selection tool can manipulate to arrive at the ideal Pareto frontier for a given mission mode.

\subsection{LunAR ORbit RENDEZVOUS MisSion Mode OPTIMIZATION}

The optimization scheme outlined in Chapter 3 and validated in Chapter 4 is implemented into a lunar architecture selection tool to simulate the Apollo Lunar Orbit Rendezvous mission mode. The design variables above are optimized via a genetic algorithm to find the optimal OEC for a given weighting. This weighting on the OEC is then systematically changed from $100 \%$ reliability-centric to $100 \%$ cost-centric. Each

optimized point is then plotted to produce a Pareto frontier of the Apollo LOR architecture. The optimized Pareto frontier for 10 different weightings of the OEC is given as Figure 57. The actual inputs chosen for by the genetic algorithm represented by the points on the Pareto frontier is given as Figure 58. 


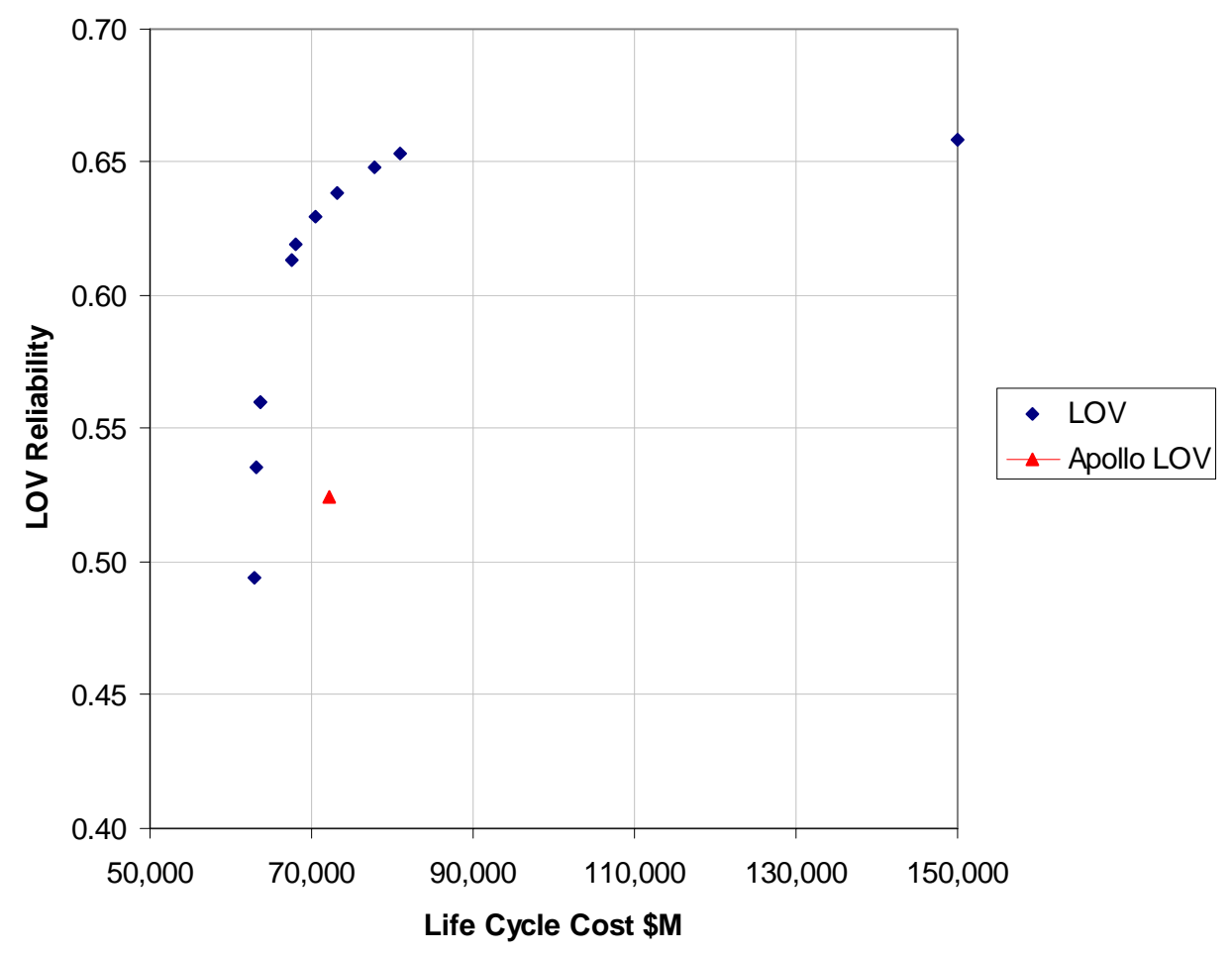

Figure 57: Pareto Frontier of the Apollo LOR Architecture

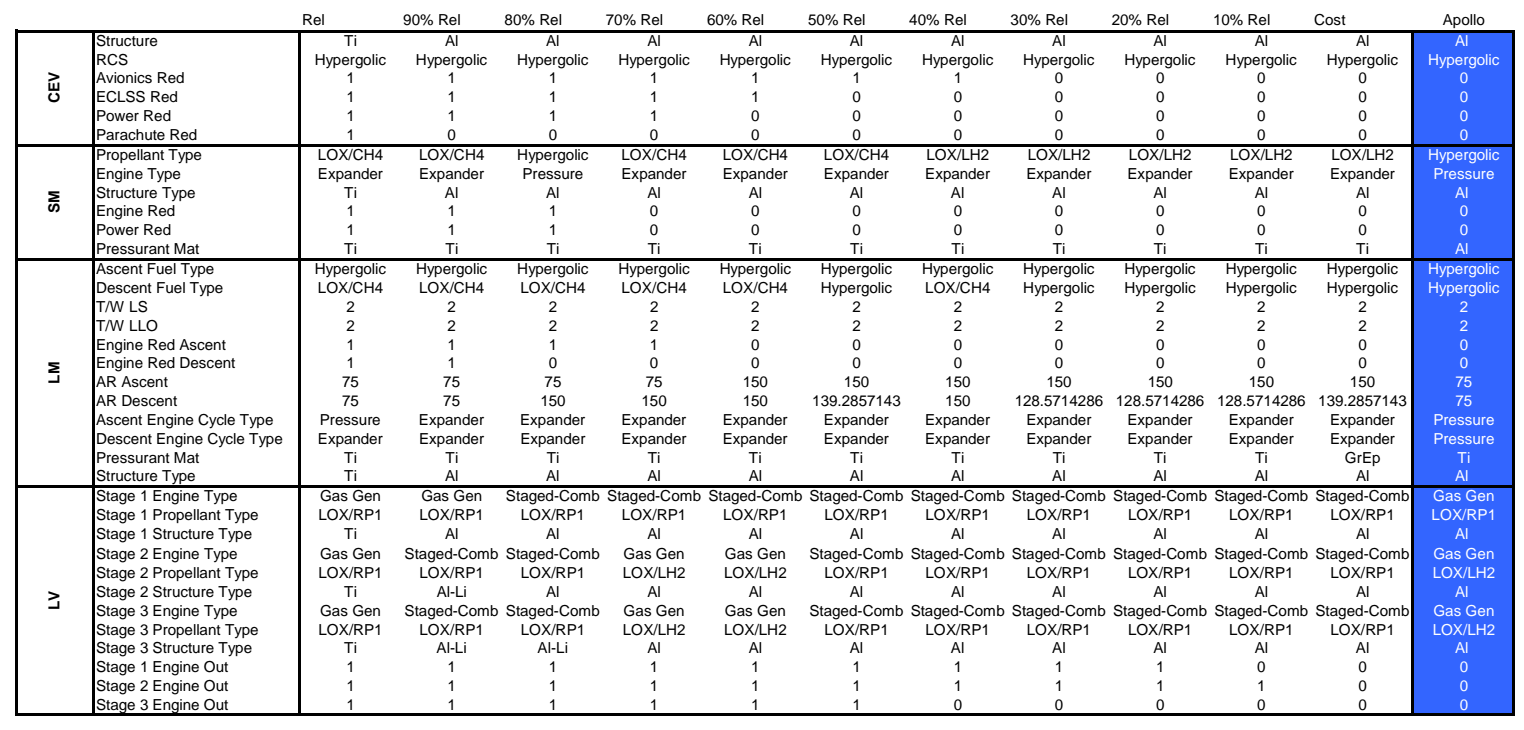

Figure 58: Summary of GA Produced Inputs for Apollo LOR Architecture 
This Pareto frontier shows the expected dimensioning returns shape of increased reliability as a function of cost. The summary of the inputs shows how the different configuration choices are made as the weighting for the OEC changes. Some interesting observations can be made from the changing inputs driven by the GA and the weighting on the OEC. First, the redundancy changes dramatically as the weighting on the OEC changes. In the $100 \%$ reliability-centric optimization, redundancy exists on all 11 elements of the architecture. This is expected since the solution is independent of cost and therefore any increase in reliability (especially through redundancy) is chosen. This $100 \%$ reliability centric model utilizes mostly titanium structures in the vehicle designs. This is because titanium results in a lighter vehicle and with lower thrust engines, and therefore a higher reliability. Propellant types vary throughout the architecture vehicles, but LOX/CH4 and the hypergolic propellants are most prevalent. Methane becomes an option because it offers higher performance (smaller engines with greater reliability) than hypergolic propellants, yet is more reliable than hydrogen due to the increased density of the fuel.

As the weighting increased towards $100 \%$ cost-centric, the propellant and structure types change as well as the redundancy in the system. The all cost optimization results in an all aluminum main structure with higher strength materials for the high pressure pressurant tanks. These structures are chosen because Al is the cheapest material per pound, while the higher strength materials are needed for the pressurant tanks due to the high weight (and cost) associated with the use of aluminum for those tanks. 
It is interesting to look at the results of the changes in redundancy as the weighting on the $\mathrm{OEC}$ is changed from $0 \%$ reliability-centric to $100 \%$ reliability-centric. As expected, there are no redundant components in the $0 \%$ reliability-centric weighting. As the weighting increases to $10 \%$, redundancy is added to the second stage of the Saturn launch vehicle. This is the first element of the architecture to gain redundancy. This is the case because of a balance between the reliability gained and the cost in mass and actual dollars of adding a redundant component. The second stage engine is added first because the engines on the second stage are generally lower reliability than that of the first stage engines (due to air start) and have a lower reliability than the third stage, due to the fact that 5 engines are operating instead of 1 engine on the third stage.

The second element to gain redundancy is the first stage of the Saturn launch vehicle. This occurs because of the fact that there are again 5 engines operating and the addition of a redundant engine adds significantly to the reliability of the system. This also occurs because there is very little mass penalty for adding a first stage engine because that extra weight is only carried by the first stage.

As the weighting is increased more component and subsystem redundancies are added as a compromise between weight, cost, and reliability. A general rule is that the lower reliability of the system the earlier a redundant component is going to be added. Unfortunately it is not that simple, since an extra pound on the CM does translates too many extra pounds on the launch vehicle due to the gear ratio of the architecture. This gear ratio simply states that an extra pound on the $\mathrm{CM}$, must be pushed by the SM which adds several extra pounds, which must then be launched which adds pounds exponentially according to the rocket equation (equation 9). This exponential 
relationship greatly affects the mass and therefore cost of the system. This gear ratio for the Apollo LOR components is demonstrated in Table 51.

Table 51: Gear Ratio for Apollo LOR Architecture Elements.

\begin{tabular}{lcccccc}
\hline & $\begin{array}{c}C M \\
(+1000 \mathrm{~kg})\end{array}$ & $\begin{array}{c}S M \\
(+1000 \mathrm{~kg})\end{array}$ & $\begin{array}{c}\text { LM } \\
(+1000 \mathrm{~kg})\end{array}$ & $\begin{array}{c}S-I V B \\
(+1000 \mathrm{~kg})\end{array}$ & $\begin{array}{c}S-I I \\
(+1000 \mathrm{~kg})\end{array}$ & $\begin{array}{c}S-I-C \\
(+1000 \mathrm{~kg})\end{array}$ \\
$\mathrm{CM}$ & $1,167 \mathrm{~kg}$ & $0 \mathrm{~kg}$ & $0 \mathrm{~kg}$ & $0 \mathrm{~kg}$ & $0 \mathrm{~kg}$ & $0 \mathrm{~kg}$ \\
SM & $1,697 \mathrm{~kg}$ & $3,595 \mathrm{~kg}$ & $3,614 \mathrm{~kg}$ & $0 \mathrm{~kg}$ & $0 \mathrm{~kg}$ & $0 \mathrm{~kg}$ \\
LM & $0 \mathrm{~kg}$ & $0 \mathrm{~kg}$ & $7,521 \mathrm{~kg}$ & $0 \mathrm{~kg}$ & $0 \mathrm{~kg}$ & $0 \mathrm{~kg}$ \\
S-I-C & $127,400 \mathrm{~kg}$ & $125,100 \mathrm{~kg}$ & $454,900 \mathrm{~kg}$ & $34,780 \mathrm{~kg}$ & $11,310 \mathrm{~kg}$ & $4,312 \mathrm{~kg}$ \\
S-II & $27,200 \mathrm{~kg}$ & $26,700 \mathrm{~kg}$ & $103,000 \mathrm{~kg}$ & $7,427 \mathrm{~kg}$ & $3,415 \mathrm{~kg}$ & $0 \mathrm{~kg}$ \\
S-IVB & $7,600 \mathrm{~kg}$ & $7,460 \mathrm{~kg}$ & $23,120 \mathrm{~kg}$ & $3,075 \mathrm{~kg}$ & $0 \mathrm{~kg}$ & $0 \mathrm{~kg}$ \\
Total & $165,000 \mathrm{~kg}$ & $162,800 \mathrm{~kg}$ & $592,200 \mathrm{~kg}$ & $45,280 \mathrm{~kg}$ & $14,730 \mathrm{~kg}$ & $4,312 \mathrm{~kg}$ \\
GR & 165 & 163 & 592 & 45 & 15 & 4 \\
\hline
\end{tabular}

This table demonstrates the effect of $1000 \mathrm{~kg}$ of dry weight growth on a vehicle element and the resulting stack growth. The gear ratio is highest for the lunar module. This is because of the high $\Delta \mathrm{V}$ that must be performed by the stages as well as the fact that the LM is payload for both the SM and the Saturn V elements. Because the CM is not payload for the lunar module, but is for the SM and the Saturn lowers the gear ratio when compared to the LM. The CM gear ratio is higher than that of the SM because of the fact that the $\mathrm{CM}$ is carried as payload of the CM. The gear ratio of the $\mathrm{CM}$ is not higher because of the fact that the $\mathrm{CM}$ has little onboard propellant, so the increase in dry weight has a small affect on the gross weight of the CM. The Saturn stages have increasing gear ratios as you increase the stage of the vehicle. As with the LM, this is because the uppers stages act as payload for the lower stages. This table shows that a pound growth on the LM has the greatest impact on the total vehicle stack for the Apollo LOR architecture. 
This directly impacts the order of increasing subsystem redundancy in the Apollo LOR architecture. A summary of the increase of redundant components as the weighting on the OEC becomes more reliability-centric is shown as Figure 59.

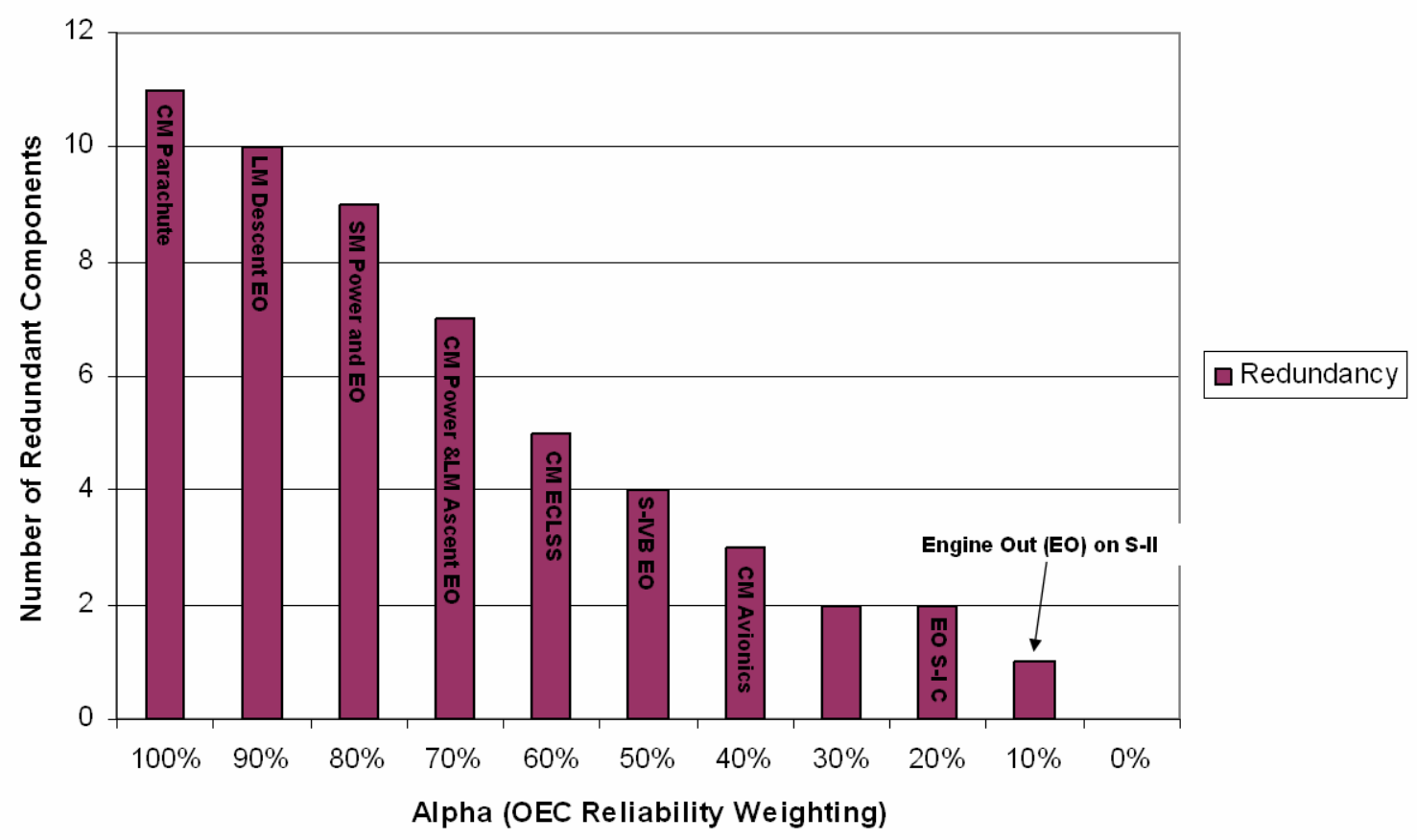

Figure 59: Redundancy in the Apollo LOR Pareto Frontier

As figure shows, the redundancy steadily increases to the maximum of eleven redundant components as the weighting on the reliability increases. The final redundant component added is the parachute redundancy. This is the last redundant component added because the descent system for the CM is generally considered a high reliability system and an extra parachute adds a significant weight to the top of the lunar architecture.

As noted in Figure 57, the optimal Pareto frontier is significantly higher reliability and lower cost than the baseline Apollo architecture. These optimal points are able to retrieve higher reliabilities and lower costs than the baseline Apollo because of the 
constraints placed on the Apollo project. Chapter 1 discussed how the Apollo lunar mode decision was made under intense schedule pressure placed on it by the John F. Kennedy's decree that the Apollo program would send a man to the moon before the end of the decade. This schedule constraint is not modeled in this methodology and therefore pushes the Apollo LOR Pareto frontier optimal past the Apollo baseline. The schedule based design choices of hypergolic propellants and limited redundancy pushed the Apollo baseline off the optimal result. A comparison of the Apollo baseline to the optimal reliability Pareto point for the same Apollo cost, and the optimal cost point to the Apollo cost is given as Figure 60 .

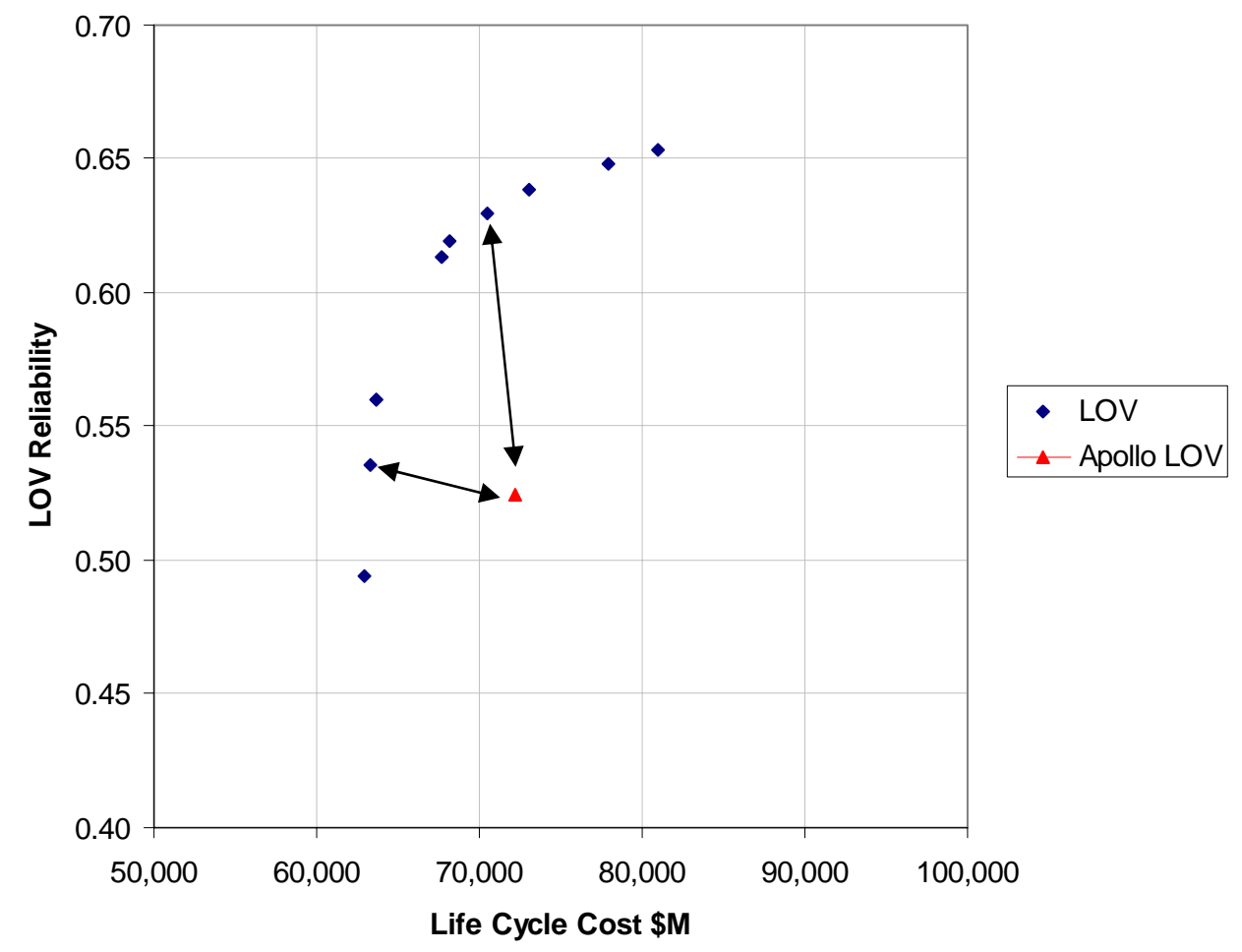

Figure 60: Comparison of Apollo Baseline to Pareto Frontier Optimal Points 
Table 52: GA Input Differences Between Apollo Baseline and Pareto Frontier

\begin{tabular}{llccc}
\hline & & $\begin{array}{c}\text { Improved } \\
\text { Reliability }\end{array}$ & $\begin{array}{c}\text { Improved } \\
\text { Cost }\end{array}$ & Apollo \\
\hline \multirow{3}{*}{ Avionics Red } & 1 & 0 & 0 \\
& ECLSS Red & 1 & 0 & 0 \\
& Power Red & 1 & 0 & 0 \\
\hline \multirow{5}{*}{ Propellant Type } & LOX/CH4 & LOX/LH2 & Hypergolic \\
Engine Type & Expander & Expander & Pressure \\
\hline Descent Fuel Type & LOX/CH4 & Hypergolic & Hypergolic \\
Engine Red Ascent & 1 & 0 & 0 \\
Engine Red Descent & 0 & 0 & 0 \\
AR Ascent & 75 & 150 & 75 \\
AR Descent & 150 & 128.5714286 & 75 \\
Ascent Engine Cycle Type & Expander & Expander & Pressure \\
Descent Engine Cycle Type & Expander & Expander & Pressure \\
\hline Stage 1 Engine Type & Staged-Comb & Staged-Comb & Gas Gen \\
Stage 1 Propellant Type & LOX/RP1 & LOX/RP1 & LOX/RP1 \\
Stage 1 Structure Type & Al & Al & Al \\
Stage 2 Engine Type & Gas Gen & Staged-Comb & Gas Gen \\
Stage 2 Propellant Type & LOX/LH2 & LOX/RP1 & LOX/LH2 \\
Stage 2 Structure Type & Al & Al & Al \\
Stage 3 Engine Type & Gas Gen & Staged-Comb & Gas Gen \\
Stage 3 Propellant Type & LOX/LH2 & LOX/RP1 & LOX/LH2 \\
Stage 3 Structure Type & Al & Al & Al \\
Stage 1 Engine Out & 1 & 1 & 0 \\
Stage 2 Engine Out & 1 & 1 & 0 \\
Stage 3 Engine Out & 1 & 0 & 0 \\
\hline
\end{tabular}


Figure 60 demonstrates that a cost improvement of almost \$9 B FY2004 dollars can be saved over the 20 year life of the program, while keeping the same reliability as the baseline Apollo architecture. If the life cycle cost was kept constant, an increase in LOM reliability of 0.1137 can be obtained. This also translates into an increase in LOC safety of 0.0803 .

As with reliability, LOC safety Pareto frontier can also be created. The Pareto frontier is very similar to the curve calculated for LOM since the LOM and LOC are similar for a given architecture. To calculate this Pareto frontier, the LOC safety numbers were calculated for the given LOM Pareto frontier points. The simulation could be reoptimized for LOC, but since the dependencies on LOM and LOC are interrelated and the Pareto frontier is of similar shape, the LOC of the optimized LOM points was used for the Pareto frontier. The Pareto frontier for LOC is included as Figure 61.

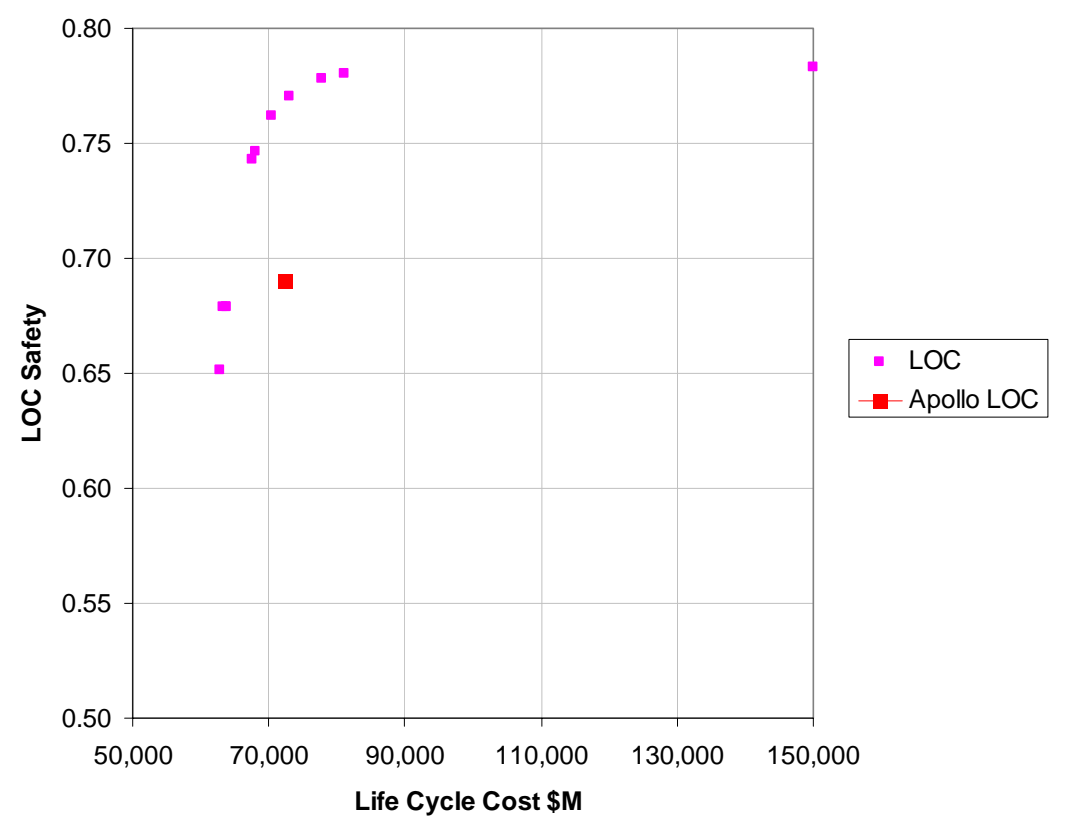

Figure 61: LOC Pareto Frontier for Apollo LOR 
The Pareto frontier can be translated into a single curve fit to accurately predict the optimal reliability or safety for a given life cycle cost. To fit this analysis a 3 node neural network was used to fit the Pareto frontier. The resulting $\mathrm{R}^{2}$ of this neural network was 0.962 . A summary of the resulting curve fit is given as Figure 62 .

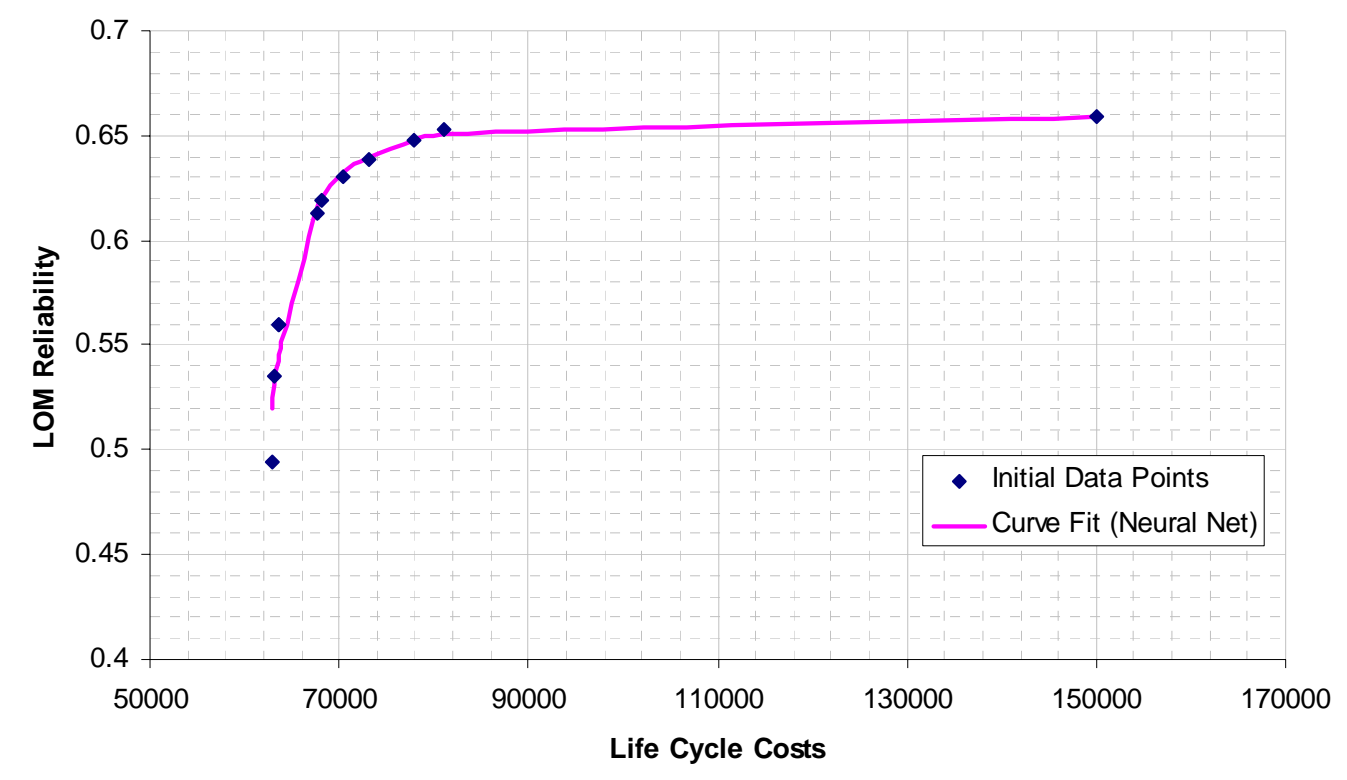

Figure 62: Neural Network Curve Fit of Pareto Frontier

An uncertainty analysis can also be performed on the optimal Pareto frontiers for both LOM and LOC. Monte Carlo simulation was completed to accomplish this uncertainty analysis. This Monte Carlo simulation was accomplished with triangular distributions on the subsystem reliabilities and catastrophic engine fractions. The resulting $90 \%$ confidence (highest cost, lowest reliability) and 10\% confidence (lowest cost, highest reliability) are plotted against the baseline results. A summary of the LOM and LOC results is given as Figure 63 and Figure 64. 




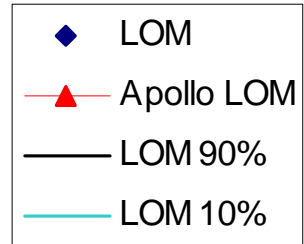

Figure 63: LOM Pareto Frontier Uncertainty

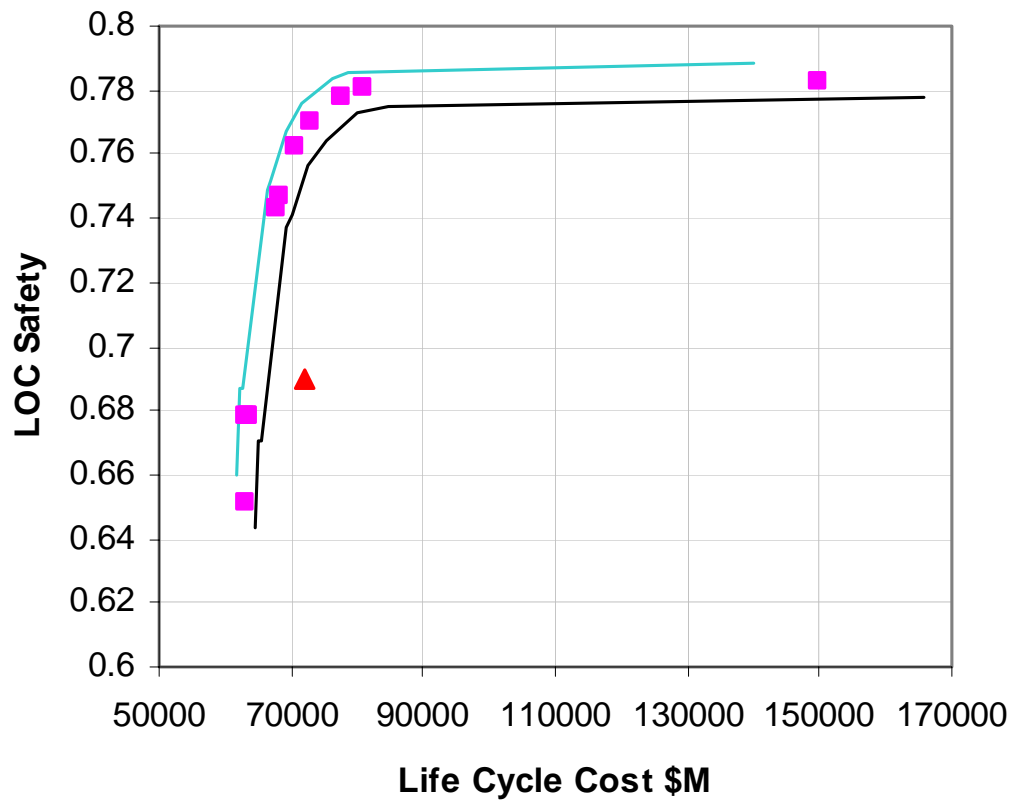

Figure 64: LOC Pareto Frontier Uncertainty 
The resulting Pareto frontiers and their uncertainties can now be presented to the decision maker to show the affect of increasing or decreasing the cost of the system affects the reliability and the safety of the system. As shown with the Apollo baseline when the decision maker is not bogged down with the details of the performance, huge savings in cost and reliability can result.

\subsection{EARTH ORBIT RENDEZVOUS MISSION MODE OPTIMIZATION}

As with the LOR, the Apollo EOR mission mode as described in Chapter 4 is optimized using the methodology outline in Chapter 3. Design variables similar to those presented in section 5.1were optimized via a genetic algorithm to find the optimal OEC for a given weighting. This weighting on the OEC is then systematically changed from $100 \%$ reliability-centric to $100 \%$ cost-centric. Each optimized point is plotted to produce a Pareto frontier of the Apollo EOR architecture. The optimized Pareto frontier for 10 different weightings of the OEC is given as Figure 57. The actual inputs chosen for by the genetic algorithm represented by the points on the Pareto frontier is given as Figure 65. 


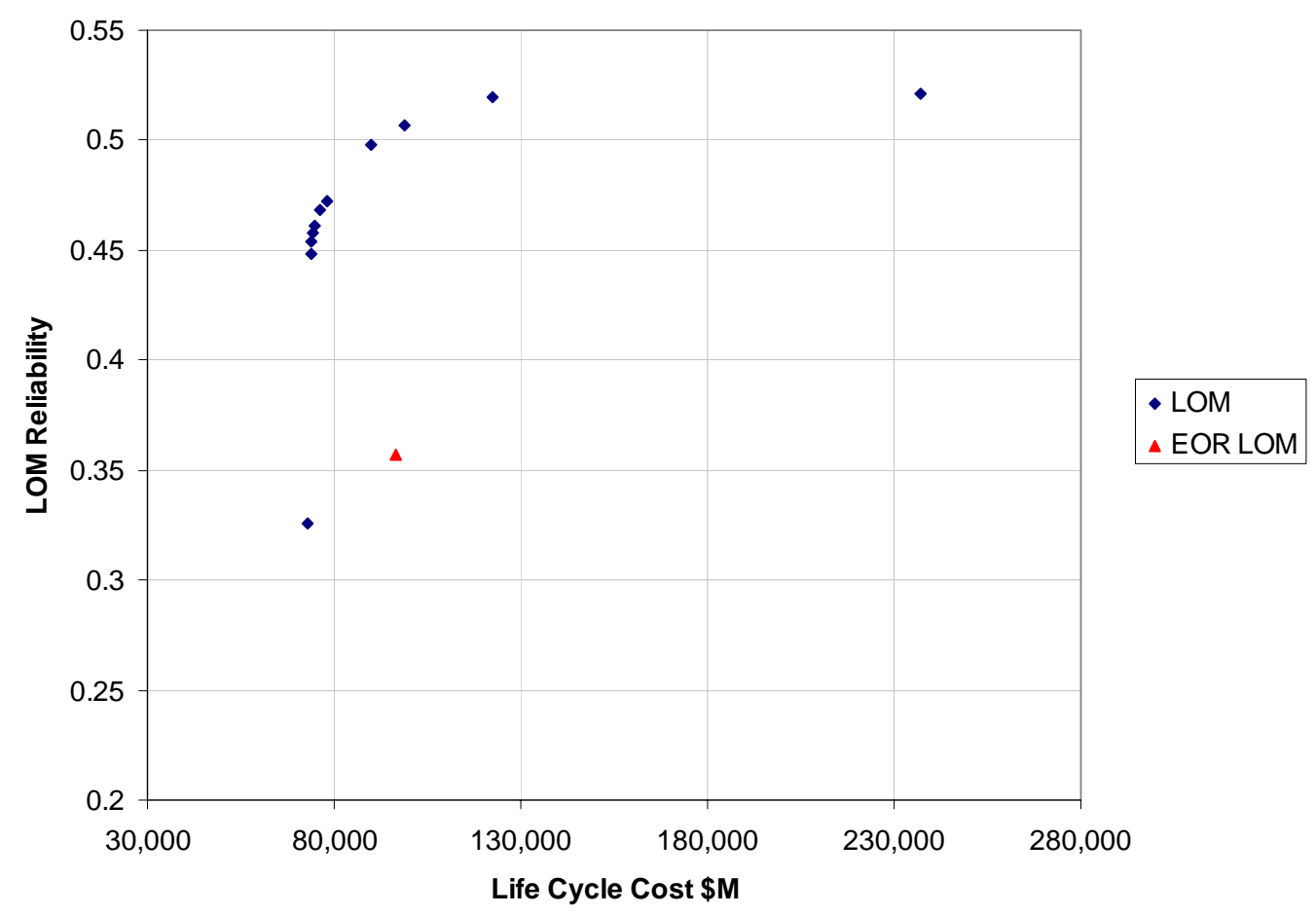

Figure 65: Pareto Frontier of the Apollo EOR Architecture

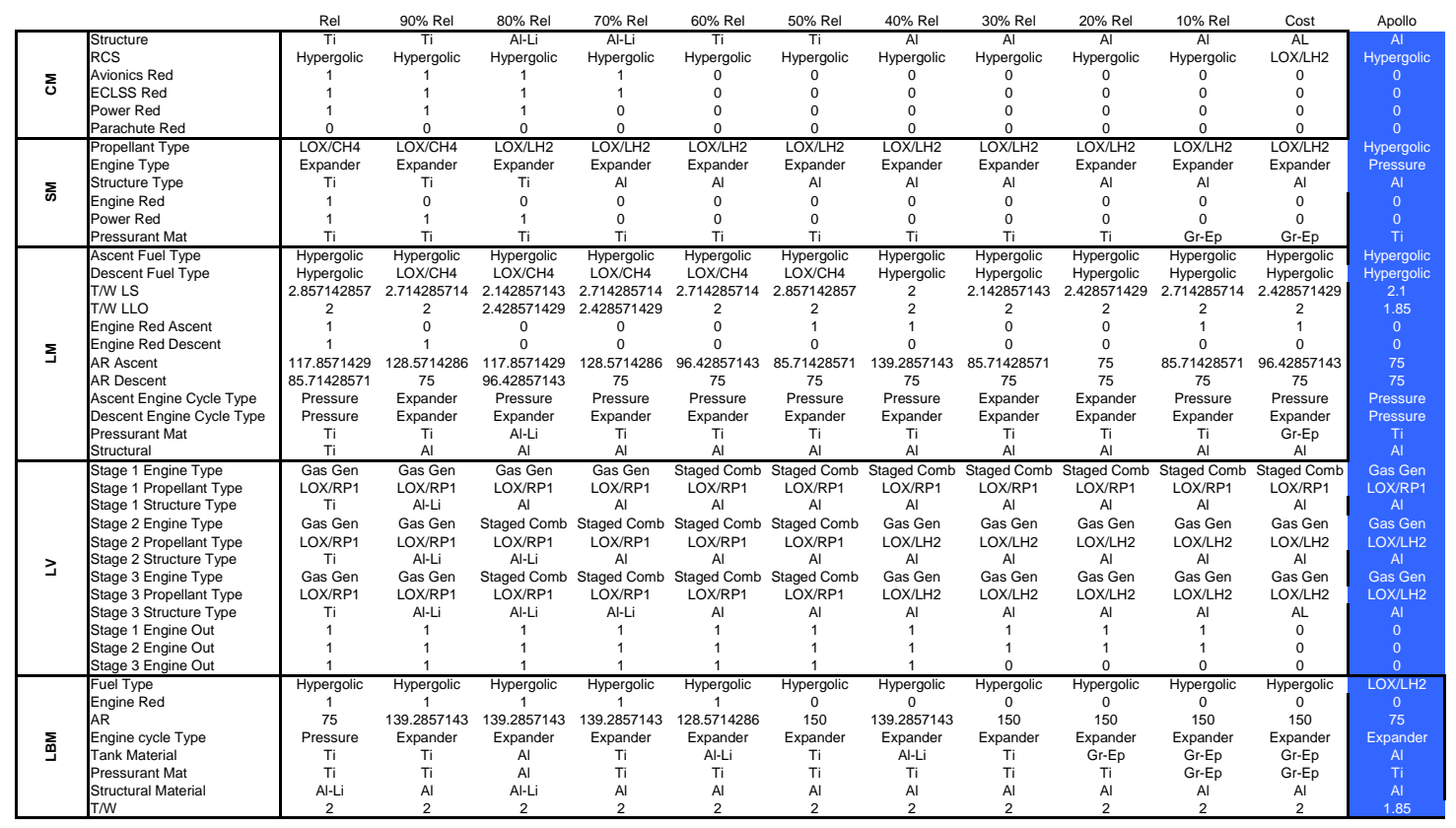

Figure 66: Summary of GA Produced Inputs for Apollo EOR Architecture 
As with the LOR architecture, this Pareto frontier of the EOR architecture shows the expected dimensioning returns shape of increased reliability as a function of cost. The summary of the inputs shows how the different configuration choices are made as the weighting for the OEC changes. As noted in Chapter 4, the main burn profiles, and therefore vehicle sizes, are significantly different between the EOR and LOR modes. The EOR model also includes a second launch vehicle and a Lunar Breaking Module (LBM). Some interesting observations can be made from the changing inputs driven by the GA and the weighting on the OEC. As with the Apollo LOR, the redundancy changes dramatically as the weighting on the OEC changes. In the $100 \%$ reliabilitycentric optimization, redundancy exists in 11 of the 12 elements of the architecture. The one redundant element which is not included is the parachute redundancy. This is because the gear ratio is higher for the EOR mission mode and the added weight of the extra parachute actually increases the size of the vehicles so much that the degradation in the other subsystem reliabilities outweighs added reliability to the parachute system. Propellant types vary throughout the architecture vehicles. LOX/LH2 is much more prevalent in the EOR system because of the higher weight penalties with taking the entire system to the lunar surface. This makes the higher performing hydrogen propellants enter into the optimized system.

It is interesting to look at the results of the changes in redundancy as the weighting on the $\mathrm{OEC}$ is changed from $0 \%$ cost-centric to $100 \%$ reliability-centric. As with the Apollo LOR architecture, there are no redundant components in the cost-centric weighting. As the weighting increases to $10 \%$, redundancy is added to both the first and second stages of the Saturn launch vehicle. This happens because of the higher 
percentage of the architecture reliability that results from the launch vehicle due to the fact that there are two separate launch vehicles in the architecture. Command module redundancy does not appear until the weighting is $70 \%$ reliability centric. This is due to the high gear ratio of the EOR architecture and the fact that the command module must be taken all the way to the lunar surface.

As with the Apollo EOR, the optimal Pareto frontier is significantly higher reliability and lower cost than the baseline EOR architecture. These optimal points are able to retrieve higher reliabilities and lower costs than the baseline Apollo because of the constraints placed on the Apollo project. A comparison of the Apollo EOR baseline to the optimal reliability Pareto point for the same Apollo EOR cost, and the optimal cost point to the Apollo cost is given as Figure 67, and the inputs to these points are given as Table 53.

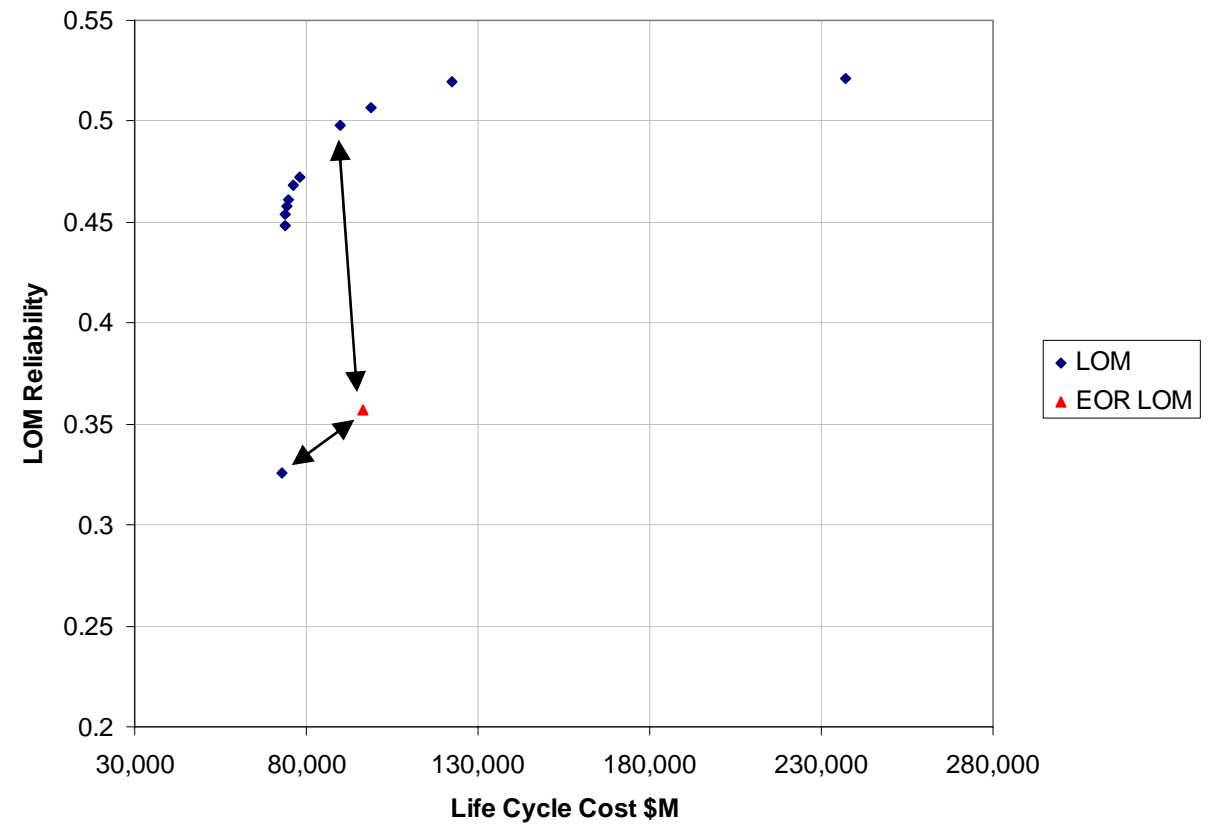

Figure 67: Comparison of Apollo EOR Baseline to Pareto Frontier Optimal Points 
Table 53: GA Input Differences Between Apollo EOR Baseline and Pareto Frontier

\begin{tabular}{|c|c|c|c|c|}
\hline & & $\begin{array}{l}\text { Improved } \\
\text { Reliability }\end{array}$ & Improved Cost & EOR \\
\hline \multirow{6}{*}{$\sum_{U}$} & Structure & Al-Li & $\overline{\mathrm{AL}}$ & $\overline{\mathrm{Al}}$ \\
\hline & $\mathrm{RCS}$ & Hypergolic & LOX/LH2 & Hypergolic \\
\hline & Avionics Red & 1 & 0 & 0 \\
\hline & ECLSS Red & 1 & 0 & 0 \\
\hline & Power Red & 0 & 0 & 0 \\
\hline & Parachute Red & 0 & 0 & 0 \\
\hline \multirow{5}{*}{$\sum_{\infty}$} & Propellant Type & LOX/LH2 & LOX/LH2 & Hypergolic \\
\hline & Engine Type & Expander & Expander & Pressure \\
\hline & Engine Red & 0 & 0 & 0 \\
\hline & Power Red & 0 & 0 & 0 \\
\hline & Pressurant Mat & $\mathrm{Ti}$ & Gr-Ep & $\mathrm{Ti}$ \\
\hline \multirow{10}{*}{$\sum_{-}$} & Ascent Fuel Type & Hypergolic & Hypergolic & Hypergolic \\
\hline & Descent Fuel Type & LOX/CH4 & Hypergolic & Hypergolic \\
\hline & T/W LS & 2.7 & 2.4 & 2.1 \\
\hline & T/W LLO & 2.4 & 2 & 1.85 \\
\hline & Engine Red Ascent & 0 & 0 & 0 \\
\hline & Engine Red Descent & 0 & 0 & 0 \\
\hline & AR Ascent & 129 & 96 & 75 \\
\hline & AR Descent & 75 & 75 & 75 \\
\hline & Descent Engine Cycle Type & Expander & Expander & Pressure \\
\hline & Pressurant Mat & $\mathrm{Ti}$ & Gr-Ep & $\mathrm{Ti}$ \\
\hline \multirow{10}{*}{ 主 } & Stage 1 Engine Type & Gas Gen & Staged Comb & Gas Gen \\
\hline & Stage 1 Propellant Type & LOX/RP1 & LOX/RP1 & LOX/RP1 \\
\hline & Stage 2 Engine Type & Staged Comb & Gas Gen & Gas Gen \\
\hline & Stage 2 Propellant Type & LOX/RP1 & LOX/LH2 & LOX/LH2 \\
\hline & Stage 3 Engine Type & Staged Comb & Gas Gen & Gas Gen \\
\hline & Stage 3 Propellant Type & LOX/RP1 & LOX/LH2 & LOX/LH2 \\
\hline & Stage 3 Structure Type & Al-Li & $\mathrm{AL}$ & $\mathrm{Al}$ \\
\hline & Stage 1 Engine Out & 1 & 0 & 0 \\
\hline & Stage 2 Engine Out & 1 & 0 & 0 \\
\hline & Stage 3 Engine Out & 1 & 0 & 0 \\
\hline \multirow{8}{*}{ 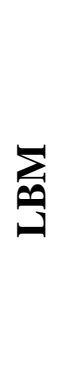 } & Fuel Type & Hypergolic & Hypergolic & LOX/LH2 \\
\hline & Engine Red & 1 & 0 & 0 \\
\hline & $\mathrm{AR}$ & 139 & 150 & 75 \\
\hline & Engine cycle Type & Expander & Expander & Expander \\
\hline & Tank Material & $\mathrm{Ti}$ & Gr-Ep & $\mathrm{Al}$ \\
\hline & Pressurant Mat & $\mathrm{Ti}$ & Gr-Ep & $\mathrm{Ti}$ \\
\hline & Structural Material & $\mathrm{Al}$ & $\mathrm{Al}$ & $\mathrm{Al}$ \\
\hline & $\mathrm{T} / \mathrm{W}$ & 2 & 2 & 1.85 \\
\hline
\end{tabular}


From Figure 67 it is shown that a cost improvement of almost \$24 B FY2004 dollars can be saved over the 20 year life of the program, while keeping approximately the same reliability as the baseline Apollo architecture. If the life cycle cost was kept constant an increase in LOM reliability of 0.1406 can be obtained. This also translates into an increase in LOC safety of 0.0512 .

As with reliability, LOC safety Pareto frontier can also be created. The Pareto frontier is very similar to the curve calculated for LOM since the LOM and LOC are similar for a given architecture. To calculate this Pareto frontier the LOC safety numbers were calculated for the given LOM Pareto frontier points. As with LOR, the EOR simulation was optimized for LOM with LOC calculated as an output. The Pareto frontier for LOC is included as Figure 68.

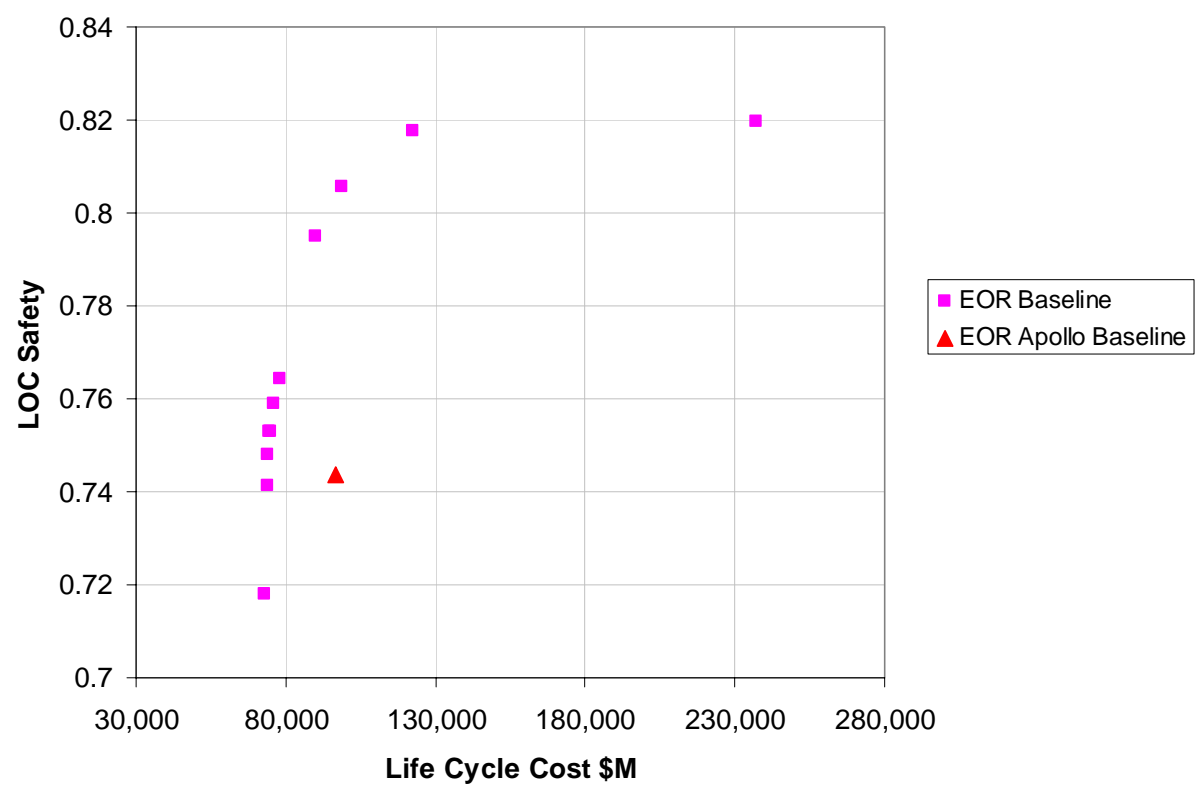

Figure 68: LOC Pareto Frontier for Apollo EOR 
As with the LOR analysis, an uncertainty analysis can also be performed on the optimal Pareto frontiers for both LOM and LOC. To accomplish this uncertainty analysis a Monte Carlo simulation was completed. This Monte Carlo simulation was accomplished with triangular distributions on the subsystem reliabilities and catastrophic engine fractions. The resulting 90\% confidence (highest cost, lowest reliability) and $10 \%$ confidence (lowest cost, highest reliability) are plotted against the baseline results. As with LOR, the uncertainties increased as the cost and reliabilities increased. This uncertainty band can then be used to determine the limits of the cost and reliabilities for the different Pareto points used for the lunar architecture selection. A summary of the LOM and LOC results is given as Figure 69 and Figure 70.

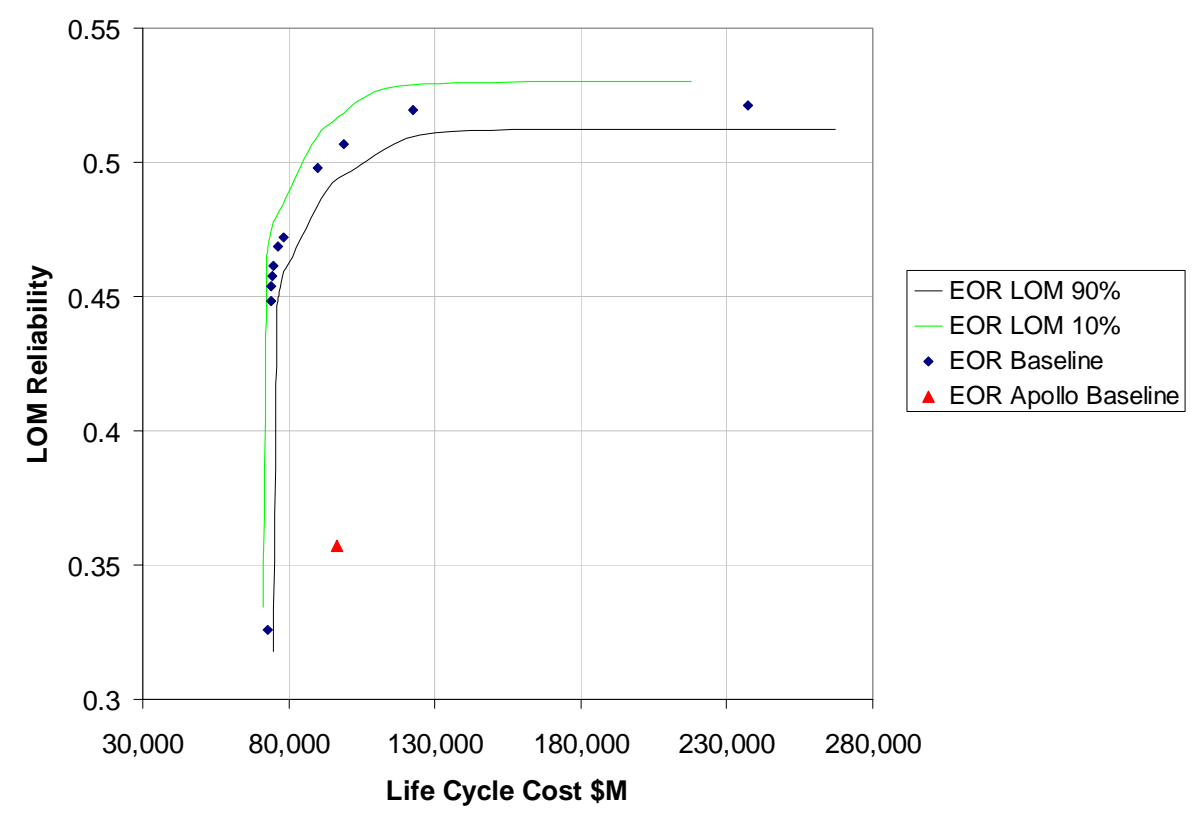

Figure 69: EOR Mission LOM Pareto Frontier Uncertainty 


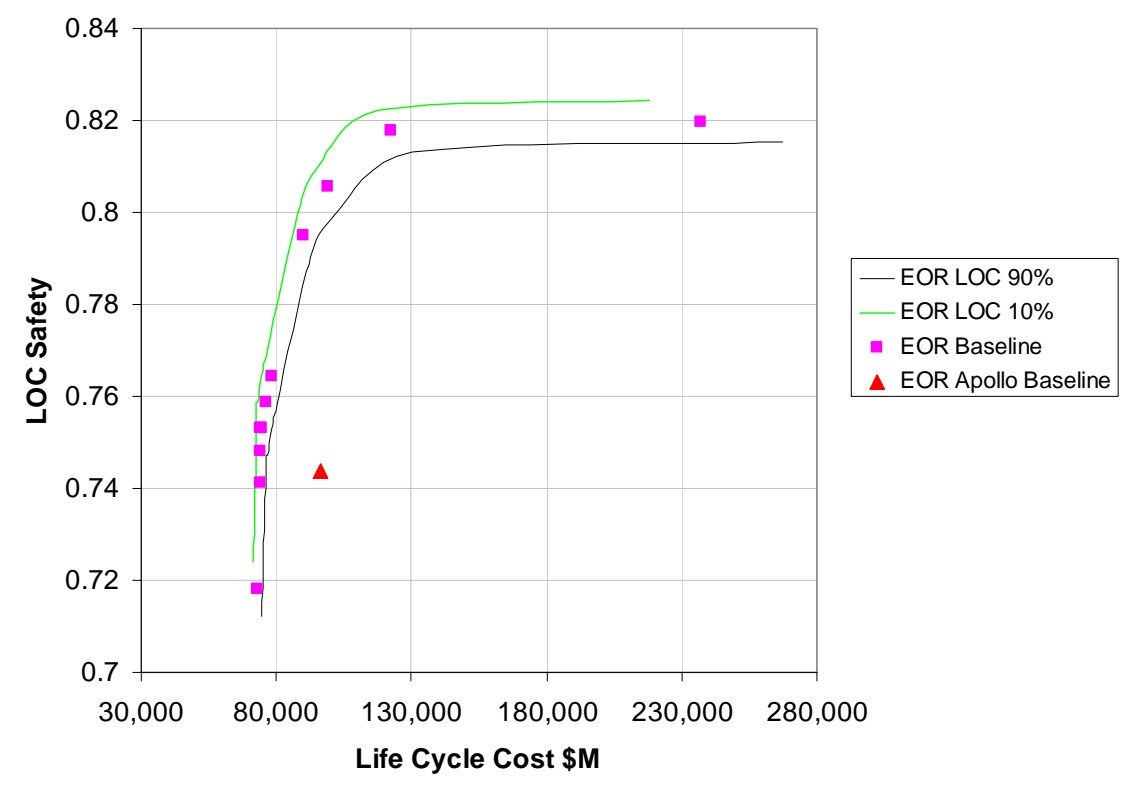

Figure 70: EOR Mission LOC Pareto Frontier Uncertainty

The resulting Pareto frontiers and their uncertainties can now be presented to the decision maker to show that increasing or decreasing the cost of the system affects the reliability and the safety of the system.

\subsection{Direct Mission Mode Optimization}

The Apollo Direct mission mode was optimized in much the same way as the Apollo LOR and EOR architectures. In fact, the Apollo Direct mission is very similar to the EOR mission except that the two launches of the EOR mission are combined into one large launch vehicle. Because of the similarities between the EOR and Direct missions, the simulations of the two modes are almost exactly the same, with the exception of the aforementioned launch vehicle ROSETTA models. The design variables similar to those presented in section 5.1 were optimized via a genetic algorithm to find the optimal OEC 
for a given weighting. This weighting on the OEC is then systematically changed from $100 \%$ reliability-centric to $100 \%$ cost-centric. Each optimized point is plotted to produce a Pareto frontier of the Apollo EOR architecture. The optimized Pareto frontier for 10 different weightings of the OEC is given as Figure 71. The actual inputs chosen for the genetic algorithm represented by the points on the Pareto frontier are given as Figure 72.

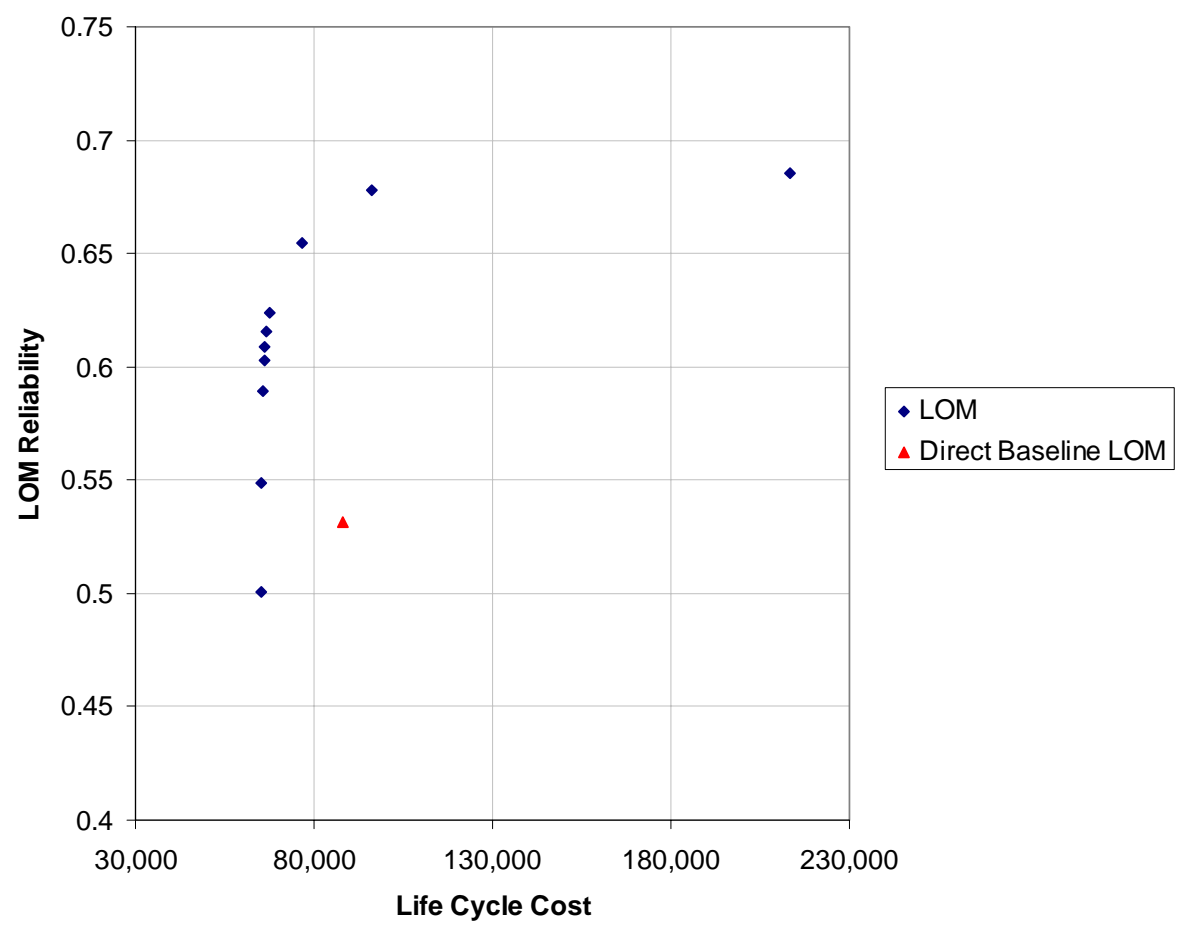

Figure 71: Pareto Frontier of the Apollo Direct Architecture 


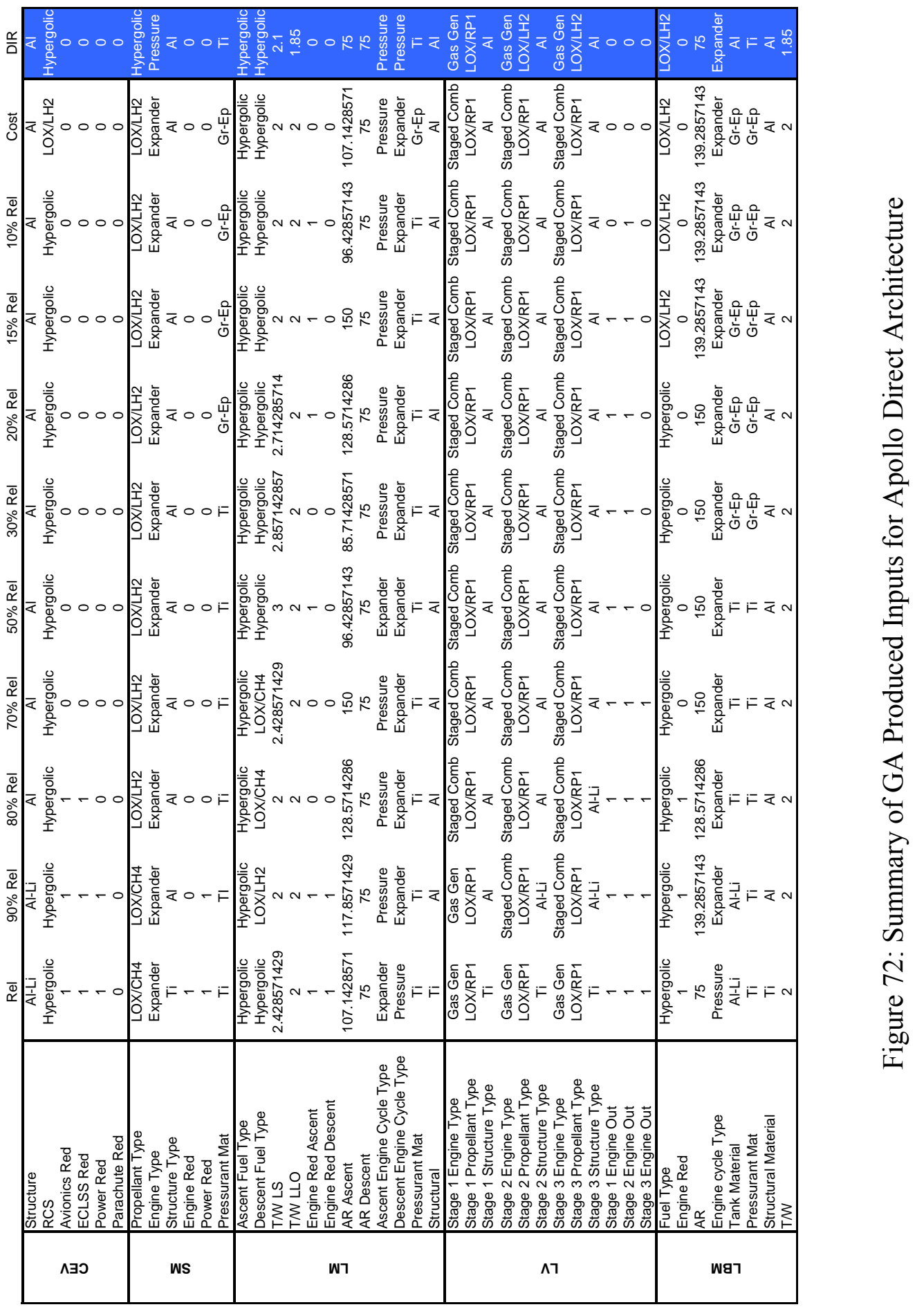


The Apollo Direct Pareto frontier has the same dimensioning returns shape as the other Apollo mission modes analyzed in this research. Some interesting observations can be made from the changing inputs driven by the GA and the weighting on the OEC. The redundancy changes dramatically as the weighting on the OEC changes. As with the Apollo EOR mission, in the $100 \%$ reliability centric optimization redundancy exists in 11 of the 12 elements of the architecture. The one excluded redundant element is the parachute redundancy. Propellant types vary throughout the architecture vehicles and LOX/LH2 is prevalent in the Direct mission due to the high gear ratio of this mission mode. The subsystem redundancy increases as the weighting on the OEC is changed from $0 \%$ cost-centric to $100 \%$ reliability-centric. The Apollo Direct mission is unique in that the LBM required LOX/LH2 propellants for the low cost solutions. This propellant choice is necessary to reduce the overall weight of the spacecraft stack and therefore the size of the Nova launch vehicle. Another interesting note in the Direct mission mode is that the Nova launch vehicle consistently uses LOX/RP1 propellants. These lower performing propellants are used because of the immense tank sizes necessary for the LOX/LH2. The LOX/RP1 requires a heavier overall vehicle, but a lower dry weight which is the variable which directly affects cost.

The optimal Pareto frontier is significantly higher reliability and lower cost than the baseline EOR architecture. These optimal points are able to retrieve higher reliabilities and lower costs than the baseline Apollo because of the constraints placed on the Apollo project. A comparison of the Apollo Direct baseline to the optimal reliability Pareto point for the same Apollo Direct cost, and the optimal cost point to the Apollo cost is given as Figure 71. 


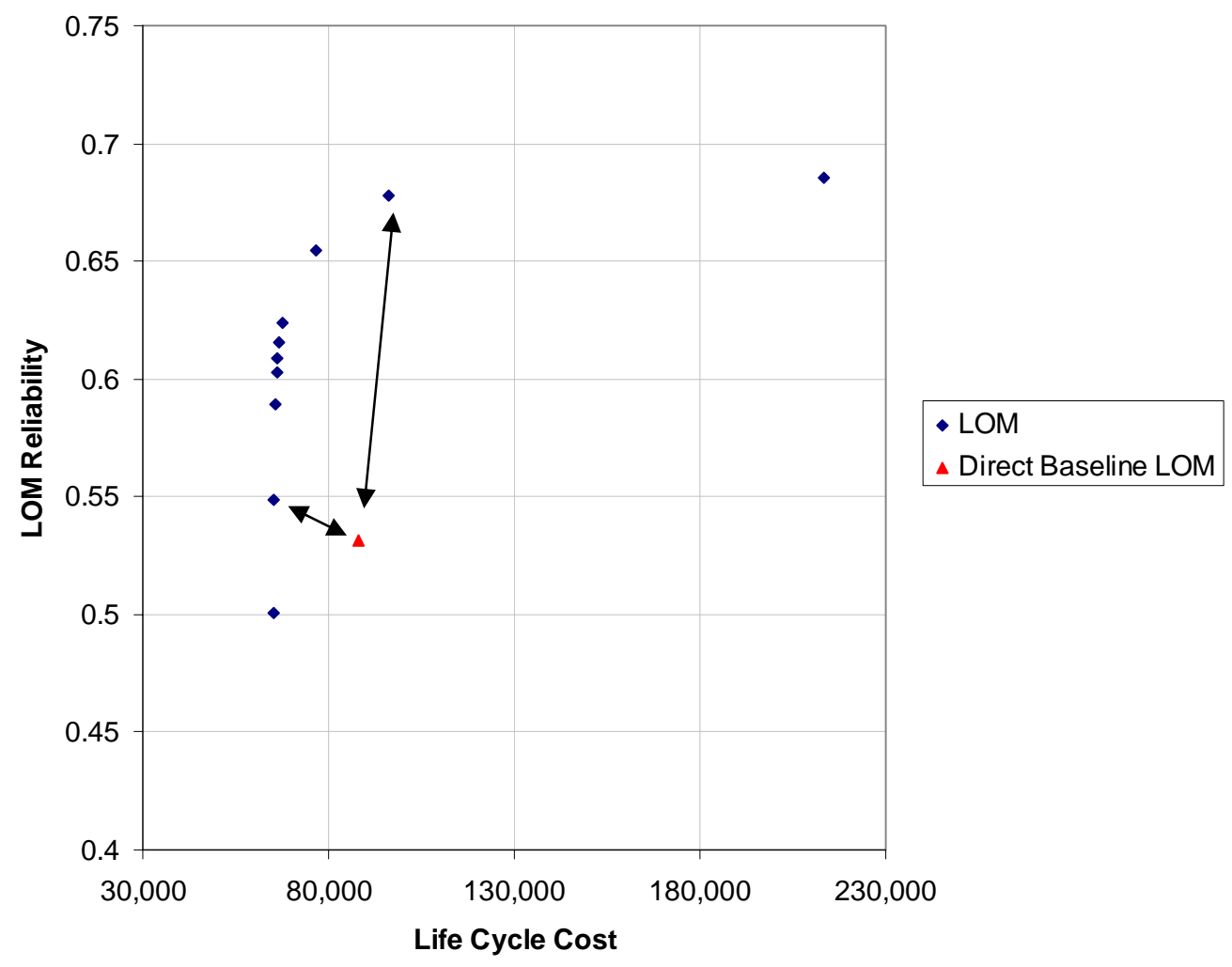

Figure 73: Comparison of Apollo Direct Baseline to Pareto Frontier Optimal Points 
Table 54: GA Input Differences Between Apollo Direct Baseline and Pareto Frontier

\begin{tabular}{|c|c|c|c|c|}
\hline & & $\begin{array}{l}\text { Increased } \\
\text { Reliability }\end{array}$ & Improved Cost & Direct \\
\hline \multirow{5}{*}{ 空 } & Structure & $\mathrm{Al}-\mathrm{Li}$ & $\overline{\mathrm{Al}}$ & $\overline{\mathrm{Al}}$ \\
\hline & $\mathrm{RCS}$ & Hypergolic & Hypergolic & Hypergolic \\
\hline & Avionics Red & 1 & 0 & 0 \\
\hline & ECLSS Red & 1 & 0 & 0 \\
\hline & Power Red & 1 & 0 & 0 \\
\hline \multirow{4}{*}{$\sum_{\infty}$} & Propellant Type & $\mathrm{LOX} / \mathrm{CH} 4$ & LOX/LH2 & Hypergolic \\
\hline & Engine Type & Expander & Expander & Pressure \\
\hline & Power Red & 1 & 0 & 0 \\
\hline & Pressurant Mat & $\mathrm{TI}$ & Gr-Ep & $\mathrm{Ti}$ \\
\hline \multirow{8}{*}{$\sum_{-1}$} & Ascent Fuel Type & Hypergolic & Hypergolic & Hypergolic \\
\hline & Descent Fuel Type & LOX/LH2 & Hypergolic & Hypergolic \\
\hline & T/W LS & 2 & 2 & 2.1 \\
\hline & T/W LLO & 2 & 2 & 1.85 \\
\hline & Engine Red Ascent & 1 & 1 & 0 \\
\hline & Engine Red Descent & 1 & 0 & 0 \\
\hline & AR Ascent & 117.8571429 & 96.42857143 & 75 \\
\hline & Descent Engine Cycle Type & Expander & Expander & Pressure \\
\hline \multirow{11}{*}{ ב } & Stage 1 Engine Type & Gas Gen & Staged Comb & Gas Gen \\
\hline & Stage 1 Propellant Type & LOX/RP1 & LOX/RP1 & LOX/RP1 \\
\hline & Stage 2 Engine Type & Staged Comb & Staged Comb & Gas Gen \\
\hline & Stage 2 Propellant Type & LOX/RP1 & LOX/RP1 & $\mathrm{LOX} / \mathrm{LH} 2$ \\
\hline & Stage 2 Structure Type & Al-Li & $\mathrm{Al}$ & $\mathrm{Al}$ \\
\hline & Stage 3 Engine Type & Staged Comb & Staged Comb & Gas Gen \\
\hline & Stage 3 Propellant Type & LOX/RP1 & $\mathrm{LOX} / \mathrm{RP} 1$ & LOX/LH2 \\
\hline & Stage 3 Structure Type & Al-Li & $\mathrm{Al}$ & $\mathrm{Al}$ \\
\hline & Stage 1 Engine Out & 1 & 0 & 0 \\
\hline & Stage 2 Engine Out & 1 & 1 & 0 \\
\hline & Stage 3 Engine Out & 1 & 0 & 0 \\
\hline \multirow{7}{*}{ 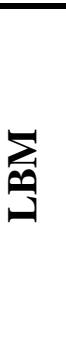 } & Fuel Type & Hypergolic & LOX/LH2 & LOX/LH2 \\
\hline & Engine Red & 1 & 0 & 0 \\
\hline & $\mathrm{AR}$ & 139.2857143 & 139.2857143 & 75 \\
\hline & Engine cycle Type & Expander & Expander & Expander \\
\hline & Tank Material & Al-Li & Gr-Ep & $\mathrm{Al}$ \\
\hline & Pressurant Mat & $\mathrm{Ti}$ & Gr-Ep & $\mathrm{Ti}$ \\
\hline & $\mathrm{T} / \mathrm{W}$ & 2 & 2 & 1.85 \\
\hline
\end{tabular}


From Figure 73 it is shown that a cost improvement of almost \$ 23 B FY2004 dollars can be saved over the 20 year life of the program while keeping approximately the same reliability as the baseline Apollo architecture. If the life cycle cost was kept constant an increase in LOM reliability of 0.1465 can be obtained. This also translates into an increase in LOC safety of 0.0722 .

As with reliability, LOC safety Pareto frontier can also be created. The Pareto frontier is very similar to the curve calculated for LOM, since the LOM and LOC are similar for a given architecture. The Pareto frontier for LOC is included as Figure 74.

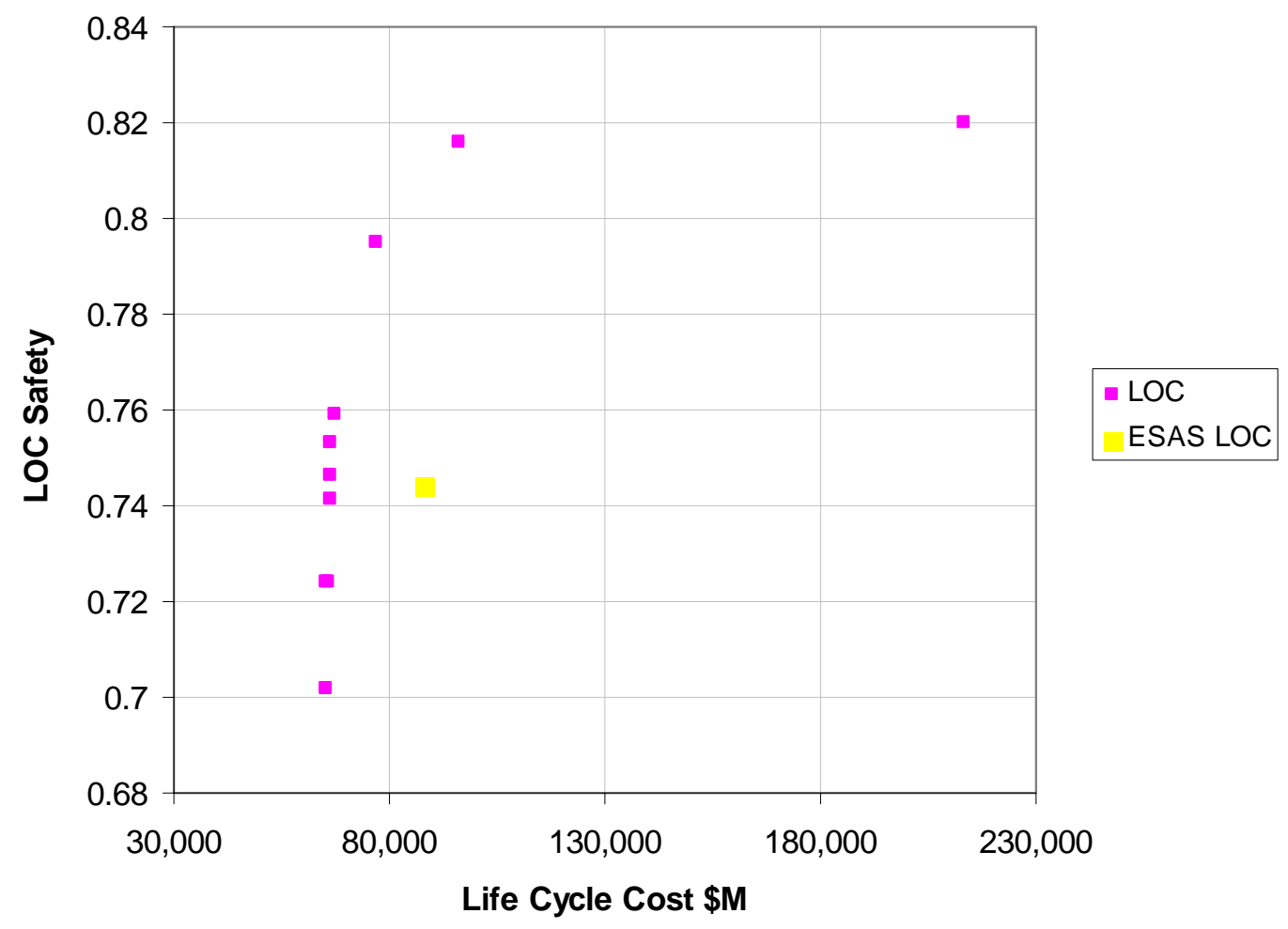

Figure 74: LOC Pareto Frontier for Apollo Direct 
This Pareto frontier is calculated from the safety analysis in the ROSETTA model on the optimized LOM Pareto frontier. This safety analysis results in a LOC of 0.8161 for the same cost as the Apollo Direct baselines LOC of 0.7440. This translates into a decrease of one loss of crew over the 20 year life of the program.

As with the other Apollo modes, an uncertainty analysis can also be performed on the optimal Pareto frontiers for both LOM and LOC. To accomplish this uncertainty analysis a Monte Carlo simulation was completed. This Monte Carlo simulation was accomplished with triangular distributions on the subsystem reliabilities and catastrophic engine fractions. The resulting 90\% confidence (highest cost, lowest reliability) and 10\% confidence (lowest cost, highest reliability) are plotted against the baseline results. A summary of the LOM and LOC results is given as Figure 75 and Figure 76.

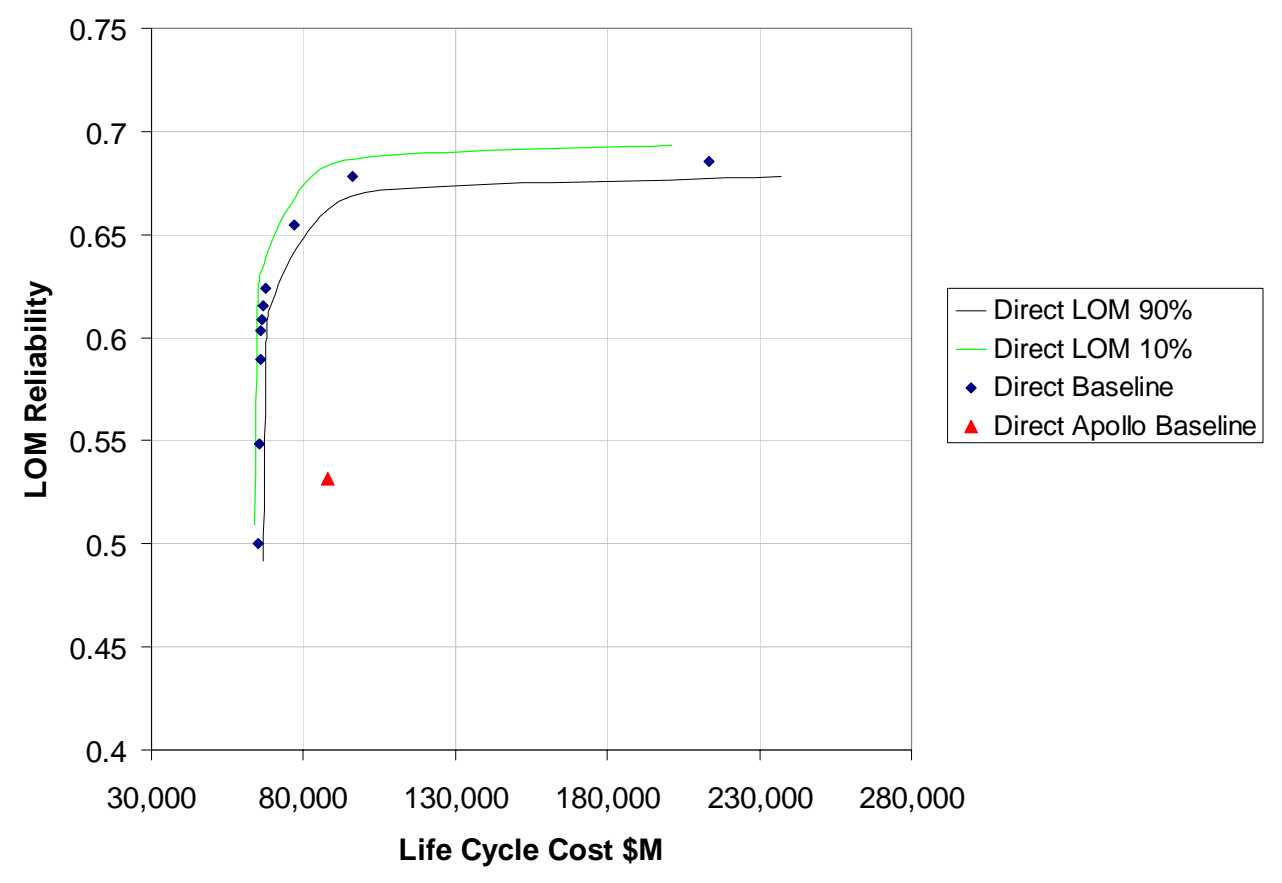

Figure 75: Direct Mission LOM Pareto Frontier Uncertainty 


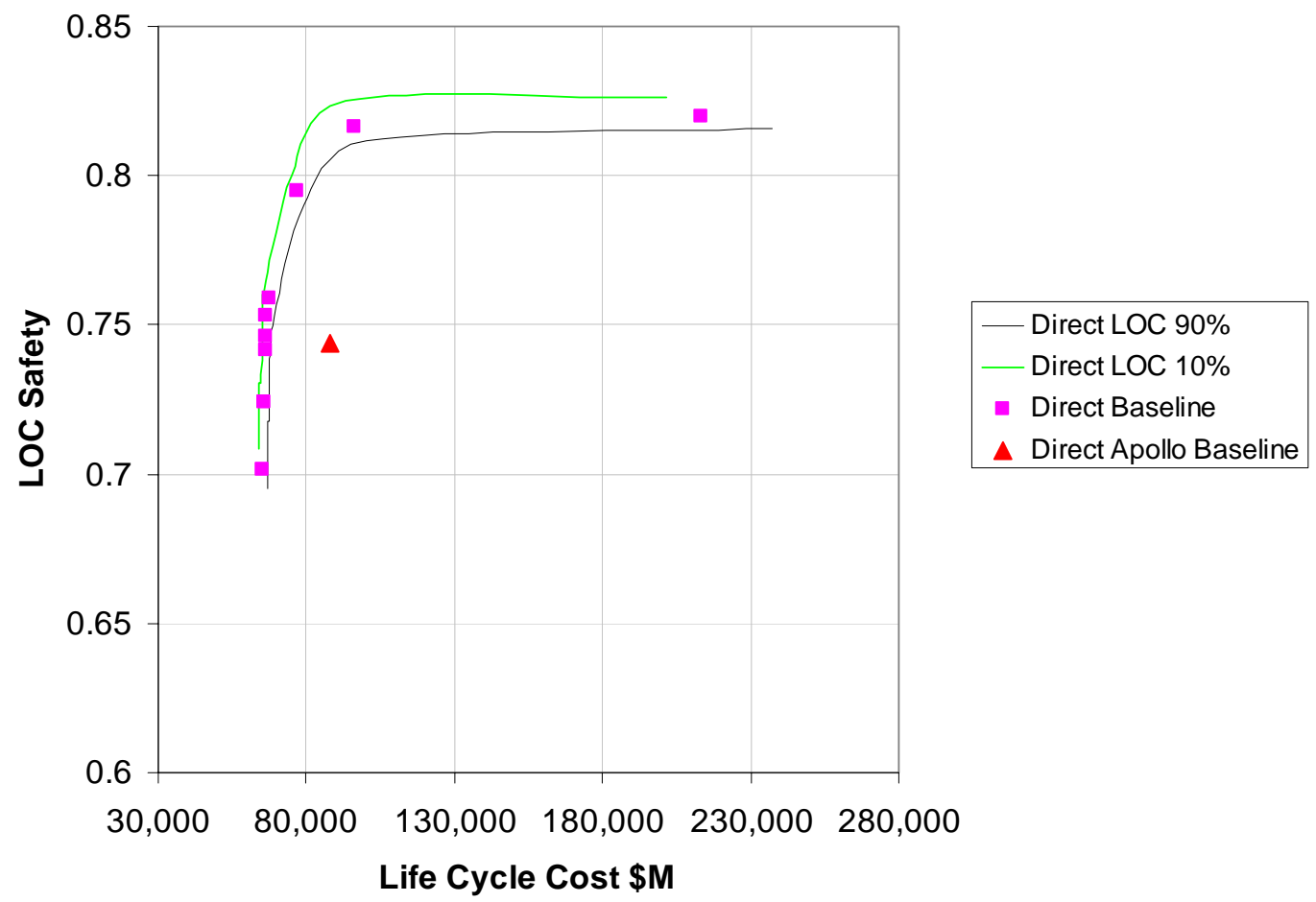

Figure 76: Direct Mission LOC Pareto Frontier Uncertainty

The uncertainty analysis provides the decision maker with some certainty about the cost and reliability that can be obtained with the optimal lunar architectures. The resulting Pareto frontiers and their uncertainties can now be presented to the decision maker to show how increasing or decreasing the cost of the system affects the reliability and the safety of the system.

\subsection{Summary OF Apollo Mission Modes}

Each of the Apollo mission modes evaluated in 1965 as a solution to the Apollo program have been evaluated with the new methodology described in this research. The Apollo analysis was reproduced in this tool, the decision reevaluated, and a series of 
optimal solutions were found. In each mode, a Pareto frontier was found that exceeded the reliability estimates and cost goals. A summary of the cost savings or reliability increases that can be achieved by evaluating points around the baseline is given as Table 55.

Table 55: Differences Between Pareto Frontiers and Apollo Baselines

\begin{tabular}{lcccccc}
\hline$\$ M$ & LOR & $\%$ Diff & EOR & $\%$ Diff & Direct & $\%$ Diff \\
LCC & $\$ 8,958$ & $12 \%$ & $\$ 23,582$ & $25 \%$ & $\$ 22,536$ & $26 \%$ \\
LOM & 0.11 & $21 \%$ & 0.14 & $39 \%$ & 0.15 & $28 \%$ \\
LOC & 0.08 & $12 \%$ & 0.05 & $7 \%$ & 0.07 & $9 \%$ \\
\hline
\end{tabular}

As this tables shows, the cost savings to achieve the same reliability as the baseline is at least $\$ 8.9$ B FY2004. The reliability gains can be on the order of $20-40 \%$ for reliability and $7-10 \%$ for safety. Cost savings of up to $36 \%$ over the Apollo baselines can be achieved with the application of this methodology.

\subsection{Current Design Practices Comparison-Exploration System}

\section{ARCHITECTURE STUdy (EOR-LOR) MISSION MODE}

Now that the each Apollo lunar mode has been evaluated, a current lunar architecture can be examined. The ESAS architecture is an EOR-LOR based mission architecture currently being evaluated in response to President Bush's Vision for Space Exploration (VSE). As described in Chapter 4, the ESAS architecture expands upon the Apollo mission with a 4 crew, 14 day lunar surface stay. The ESAS architecture was 
simulated in much the same way as the Apollo lunar modes. A summary architecture inputs is given as Table 56 .

Table 56: Architecture Inputs for the ESAS Lunar Architecture

\begin{tabular}{lc}
\hline & ESAS \\
Total Crew & 4 \\
Crew to Surface & 4 \\
Number of Lunar Flights/Yr & 2 \\
Number of Program Yrs & 20 \\
Time on Lunar Surface & 14 \\
Number of Days SM Provides Power & 6 \\
Number of Days LM Provides Power & 14 \\
Crew Escape System & $\mathrm{Y}$ \\
CM Volume/Crew & 7.95 \\
Commonality w/SM and LM Engines & $\mathrm{N}$ \\
\hline
\end{tabular}

As this table shows, the ESAS architecture inputs are more ambitious than the Apollo inputs. The uncertainty associated with the definition of the lunar campaign that will be used for the ESAS architecture resulted in the same baseline architecture of a 20 year, 32 flight campaign to be used,

The design variables for the ESAS spacecraft are exactly the same as the variables for the Apollo spacecraft. This is because the same ROSETTA models (with different burn profiles) was used. The main difference is the launch vehicle ROSETTA models. In the case of ESAS, a CLV and CaLV model was created to evaluate the launch vehicle performance, cost, and reliability. A summary of the morphological matrices for the CLV and CaLV are given as Table 57 and Table 58. 
Table 57: Morphological Matrix for Crew Launch Vehicle ROSETTA Model

\begin{tabular}{lcclll}
\hline Stage 1 Engine Type & 4-seg SRB & 5-seg SRB & & & \\
Stage 2 Engine Type & Gas Generator & Staged-Combustion & & & \\
Stage 2 Propellant Type & LOX/LH2 & LOX/RP1 & & & \\
Stage 2 Structure Type & $\mathrm{Al}$ & $\mathrm{Al}-\mathrm{Li}$ & Ti & Gr-Ep & MMC \\
Stage 2 \# Engines & 1 & 10 & & & \\
Stage 2 T/W & 0.5 & 2 & & & \\
Crew Escape System & $\mathrm{Y}$ & $\mathrm{N}$ & & & \\
EO 2nd Stage & 0 & 1 & & & \\
\hline
\end{tabular}

Table 58: Morphological Matrix for Cargo Launch Vehicle ROSETTA Model

\begin{tabular}{lcclll}
\hline Stage 1 Engine Type & 4-seg SRB & 5-seg SRB & & & \\
Core Engine Type & Gas Generator & Staged-Combustion & & & \\
Core Propellant Type & LOX/LH2 & LOX/RP1 & & & \\
Core Structure Type & $\mathrm{Al}$ & $\mathrm{Al}-\mathrm{Li}$ & $\mathrm{Ti}$ & $\mathrm{Gr}-\mathrm{Ep}$ & $\mathrm{MMC}$ \\
Core \# Engines & 1 & 10 & & & \\
Core T/W & 0.5 & 2 & & & \\
EDS Engine Type & Gas Generator & Staged-Combustion & & & \\
EDS Propellant Type & LOX/LH2 & LOX/RP1 & & & \\
EDS Structure Type & $\mathrm{Al}$ & $\mathrm{Al}-\mathrm{Li}$ & $\mathrm{Ti}$ & $\mathrm{Gr}-\mathrm{Ep}$ & $\mathrm{MMC}$ \\
EDS \# Engines & 1 & 10 & & & \\
EDS T/W & 0.5 & 2 & & & \\
Crew Escape System & $\mathrm{Y}$ & $\mathrm{N}$ & & & \\
EO Core & 0 & 1 & & & \\
EO EDS & 0 & 1 & & & \\
\hline
\end{tabular}


The design variables are optimized via a genetic algorithm to find the optimal OEC for a given weighting. This weighting on the OEC is then systematically changed from $100 \%$ reliability-centric to $100 \%$ cost-centric. Each optimized point is then plotted to produce a Pareto frontier of the ESAS architecture. The optimized Pareto frontier for 10 different weightings of the OEC is given as Figure 77. The actual inputs chosen by the genetic algorithm represented by the points on the Pareto frontier is given as Figure 78.

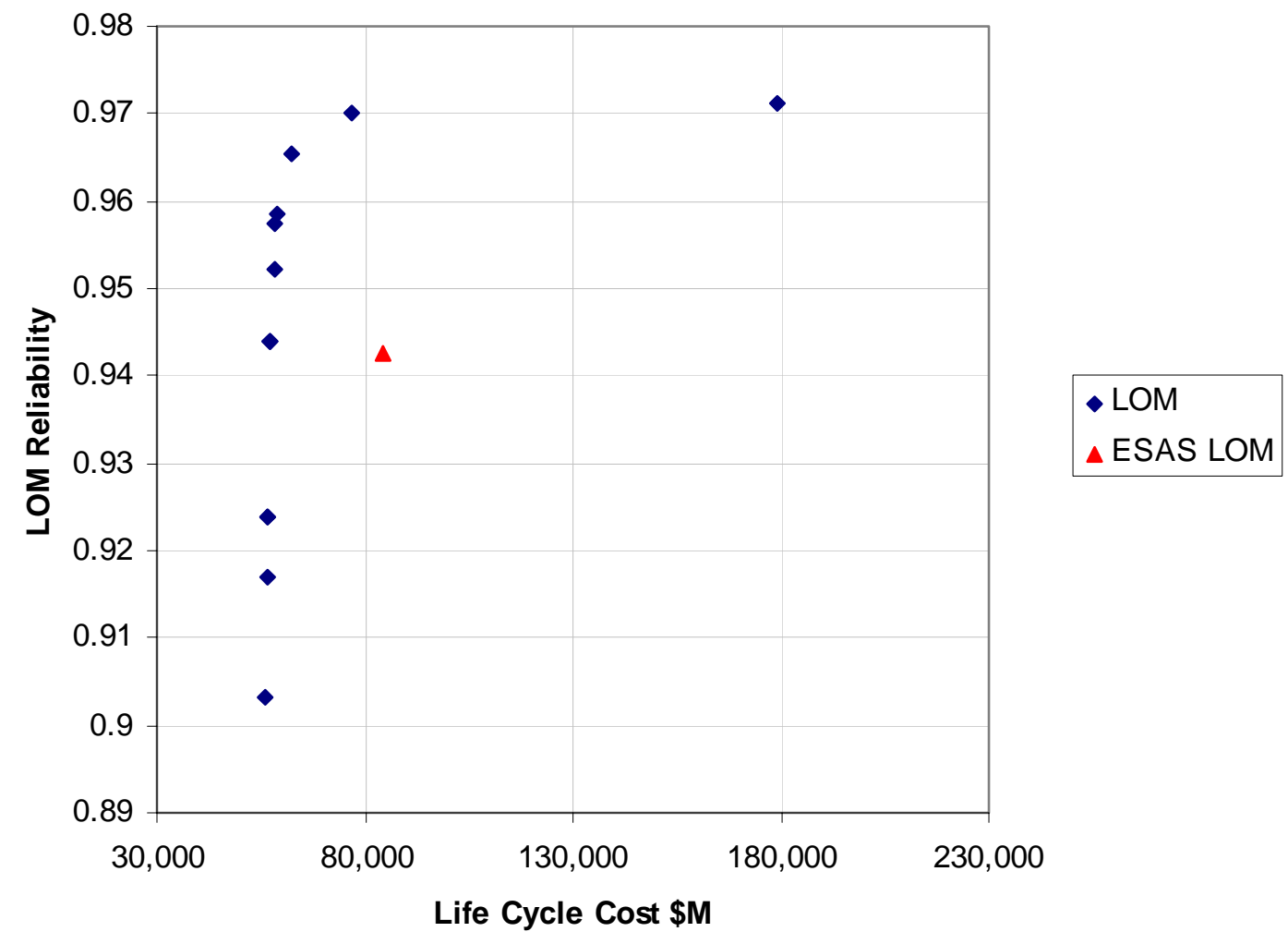

Figure 77: Pareto Frontier of the ESAS Architecture 


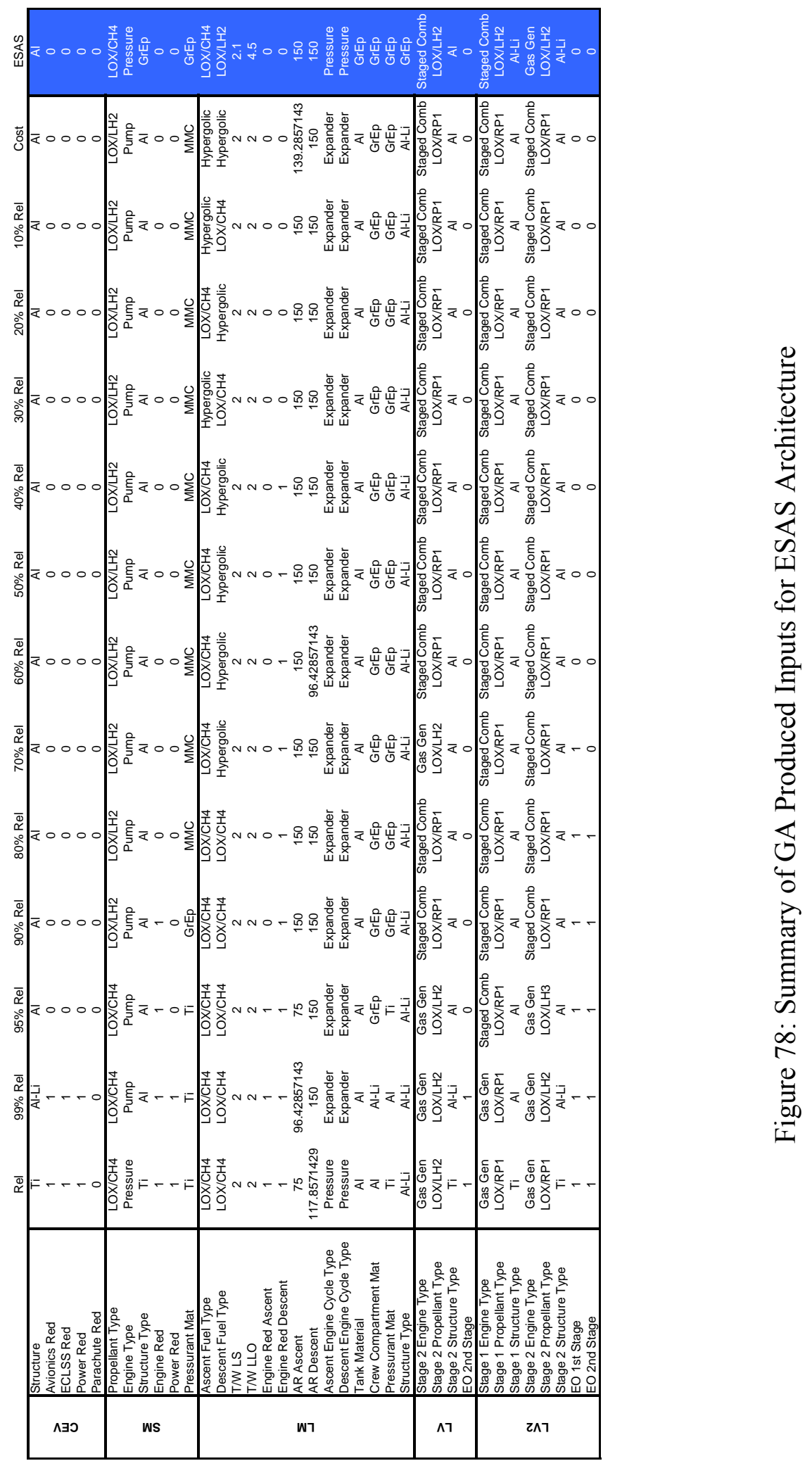


For the ESAS architecture, 13 GA points were evaluated as opposed to the standard 10 points of the Apollo architecture modes. These extra GA points were to fully fill out the Pareto frontier and add points near the ends of the Pareto frontiers. The summary of the inputs shows how the different configuration choices are made as the weighting for the OEC changes. Some interesting observations can be made from the changing inputs as driven by the GA and the weighting on the OEC. First, the redundancy disappears much earlier (lower cost weightings) than in the Apollo architectures. This is because the subsystem reliabilities are much higher for the current vehicles and thus the cost of adding redundancy is not justified.

As with the EOR and Direct Apollo architectures, the 100\% reliability-centric optimization redundancy exists on 10 of 11 elements of the architecture. The parachute redundancy is again omitted because it decreases the overall reliability of the system as shown in the EOR architecture. Launch vehicle engine redundancy first appears on the CaLV ( $1^{\text {st }}$ than $2^{\text {nd }}$ stages) and then on the CLV. This occurs because the CaLV is a completely rubberized (can parametrically scale) system, whereas the CLV is limited in scalability by the solid rocket booster first stage which is considered static. Redundancy on the CEV does not occur until the weighting is $99 \%$ reliability centric due to the high weight penalty and minimal resulting reliability increase with the already high reliability of the subsystem.

The LSAM or LM descent engine redundancy appears at a much lower reliability weighting than any of the Apollo modes. This is due to the fact that the LSAM has a four engine configuration on the descent propulsion system. This four engine system has a much lower reliability than a single engine system of the same thrust. Therefore the 
weight penalty of adding an extra engine is only a quarter of the weight of adding a similar thrust single engine system. As with Apollo, the propellant types vary throughout the architecture vehicles, but LOX/CH4 and LOX/LH2 are most prevalent. The methane and hydrogen propellants both offer a higher performance $\left(I_{\mathrm{sp}}\right)$ over the hypergolic propellants; and, because of the ambitious requirements for the ESAS architecture, the higher performance is needed. A summary of the redundancy in the ESAS architecture is given as Figure 79.

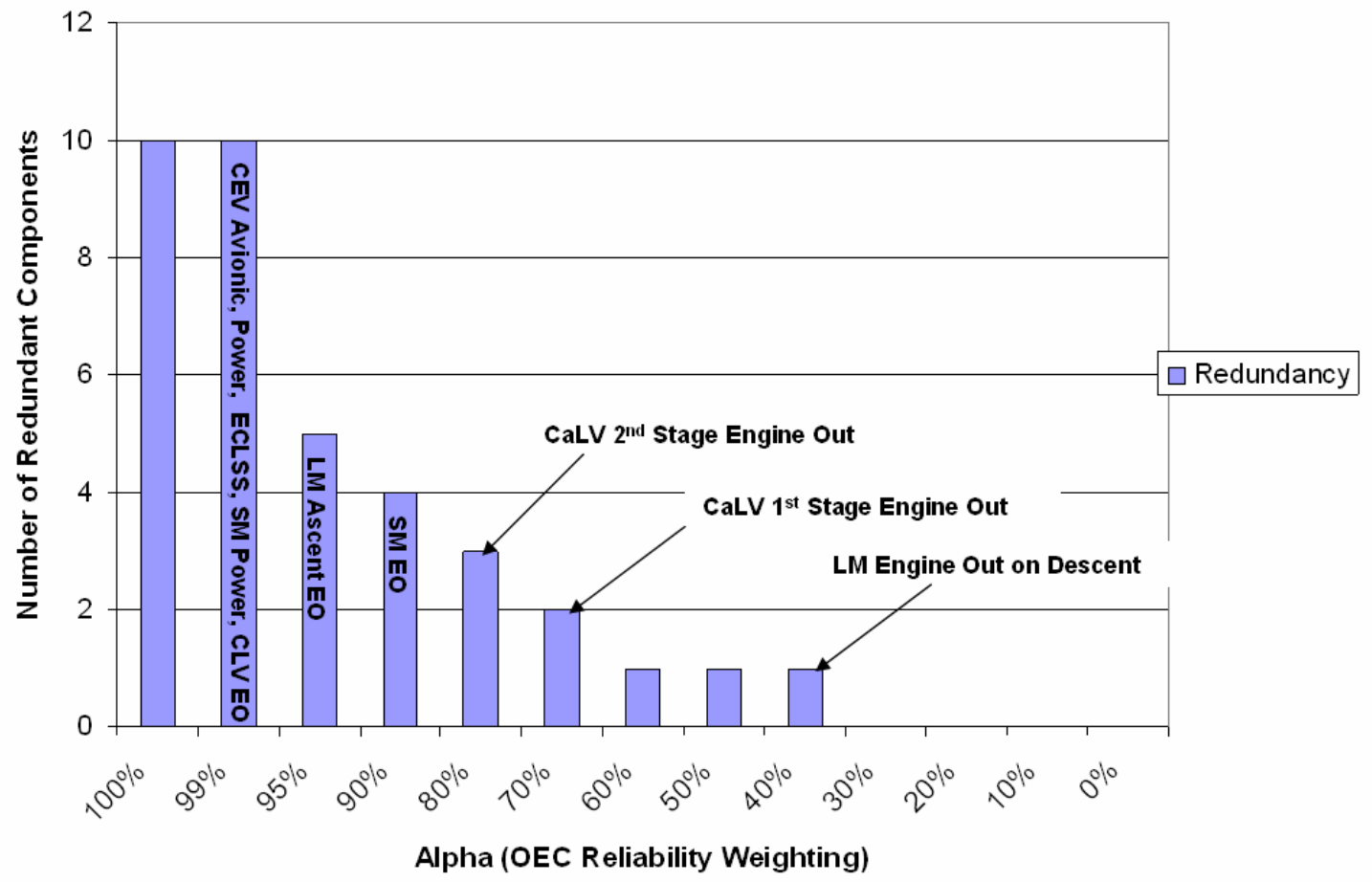

Figure 79: Redundancy in the ESAS Pareto Frontier

As this figure shows, the redundancy steadily increases to the maximum of ten redundant components as the weighting on the reliability increases. The final components added are 
the CM redundancies, due the high gear ratio of the ESAS design and therefore the high weight penalty associated with adding CEV mass to the system.

As noted in Figure 57, the optimal Pareto frontier has significantly higher reliability and lower cost than the baseline ESAS architecture. These optimal points are able to retrieve higher reliabilities and lower costs than the baseline ESAS architecture. This difference in solution could be due to political constraints placed on the ESAS project, but are more likely a result of the design process used to evaluate the ESAS baseline. As noted earlier in this thesis, the ESAS architecture team used an old fashioned philosophy of calculating baseline cases and trade studies off the baseline case. This methodology allows the entire design space to be evaluated and the best solutions chosen without the biases of the involved designers. A comparison of the Apollo baseline to the optimal reliability Pareto point for the same Apollo cost, and the optimal cost point to the Apollo cost is given as Table 59.

Figure 80 shows that a cost improvement of \$27 B FY2004 dollars can be saved over the 20 year life of the program, while keeping the same reliability as the baseline ESAS architecture. If the life cycle cost was kept constant, an increase in LOM reliability of 0.0274 can be obtained. This also translates into an increase in LOC safety of 0.0148 . The smaller increase in reliability and safety can be attributed to the already high reliability of the baseline system. In terms of flights, this small reliability increase changes the LOM from 1 in 17 flights to a 1 in 33 flights and the LOC from 1 in 25 flights to 1 in 40 flights. 


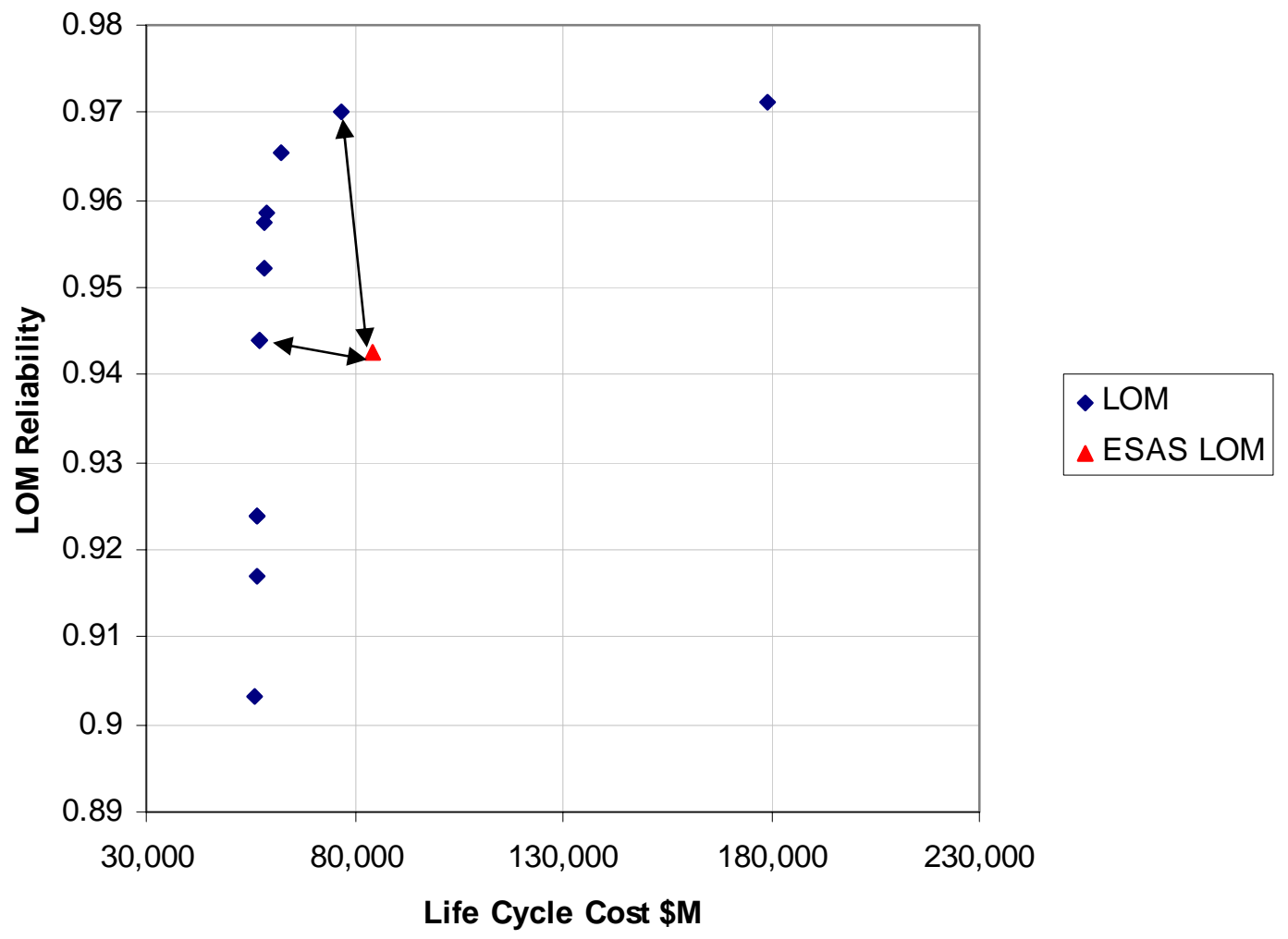

Figure 80: Comparison of ESAS Baseline to Pareto Frontier Optimal Points 
Table 59: GA Input Differences Between ESAS Baseline and Pareto Frontier

\begin{tabular}{|c|c|c|c|c|}
\hline & & $\begin{array}{l}\text { Increased } \\
\text { Reliability }\end{array}$ & $\begin{array}{c}\text { Improved } \\
\text { Cost }\end{array}$ & ESAS \\
\hline \multirow{4}{*}{ 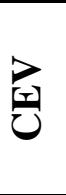 } & Structure & $\mathrm{Al}-\mathrm{Li}$ & $\mathrm{Al}$ & $\mathrm{Al}$ \\
\hline & Avionics Red & 1 & 0 & 0 \\
\hline & ECLSS Red & 1 & 0 & 0 \\
\hline & Power Red & 1 & 0 & 0 \\
\hline \multirow{6}{*}{$\sum_{\infty}$} & Propellant Type & $\mathrm{LOX} / \mathrm{CH} 4$ & LOX/LH2 & LOX/CH4 \\
\hline & Engine Type & Pump & Pump & Pressure \\
\hline & Structure Type & $\mathrm{Al}$ & $\mathrm{Al}$ & GrEp \\
\hline & Engine Red & 1 & 0 & 0 \\
\hline & Power Red & 1 & 0 & 0 \\
\hline & Pressurant Mat & $\mathrm{Ti}$ & MMC & GrEp \\
\hline \multirow{14}{*}{$\sum_{-1}$} & Ascent Fuel Type & LOX/CH4 & LOX/CH4 & LOX/CH4 \\
\hline & Descent Fuel Type & $\mathrm{LOX} / \mathrm{CH} 4$ & Hypergolic & LOX/LH2 \\
\hline & T/W LS & 2 & 2 & 2.1 \\
\hline & T/W LLO & 2 & 2 & 4.5 \\
\hline & Engine Red Ascent & 1 & 0 & 0 \\
\hline & Engine Red Descent & 1 & 1 & 0 \\
\hline & AR Ascent & 96 & 150 & 150 \\
\hline & AR Descent & 150 & 150 & 150 \\
\hline & Ascent Engine Cycle Type & Expander & Expander & Pressure \\
\hline & Descent Engine Cycle Type & Expander & Expander & Pressure \\
\hline & Tank Material & $\mathrm{Al}$ & $\mathrm{Al}$ & GrEp \\
\hline & Crew Compartment Mat & Al-Li & GrEp & GrEp \\
\hline & Pressurant Mat & $\mathrm{Al}$ & GrEp & GrEp \\
\hline & Structure Type & $\mathrm{Al}-\mathrm{Li}$ & Al-Li & GrEp \\
\hline \multirow{4}{*}{ ن } & Stage 2 Engine Type & Gas Gen & Staged Comb & Staged Comb \\
\hline & Stage 2 Propellant Type & LOX/LH2 & LOX/RP1 & LOX/LH2 \\
\hline & Stage 2 Structure Type & Al-Li & $\mathrm{Al}$ & $\mathrm{Al}$ \\
\hline & EO 2nd Stage & 1 & 0 & 0 \\
\hline \multirow{8}{*}{ ) } & Stage 1 Engine Type & Gas Gen & Staged Comb & Staged Comb \\
\hline & Stage 1 Propellant Type & LOX/RP1 & LOX/RP1 & LOX/LH2 \\
\hline & Stage 1 Structure Type & $\mathrm{Al}$ & $\mathrm{Al}$ & Al-Li \\
\hline & Stage 2 Engine Type & Gas Gen & Staged Comb & Gas Gen \\
\hline & Stage 2 Propellant Type & LOX/LH2 & LOX/RP1 & LOX/LH2 \\
\hline & Stage 2 Structure Type & Al-Li & $\mathrm{Al}$ & Al-Li \\
\hline & EO 1st Stage & 1 & 0 & 0 \\
\hline & EO 2nd Stage & 1 & 0 & 0 \\
\hline
\end{tabular}


As with reliability, a LOC safety Pareto frontier can also be created. The Pareto frontier is very similar to the curve calculated for LOM, since the LOM and LOC are similar for a given architecture. The Pareto frontier for LOC is included as Figure 81.

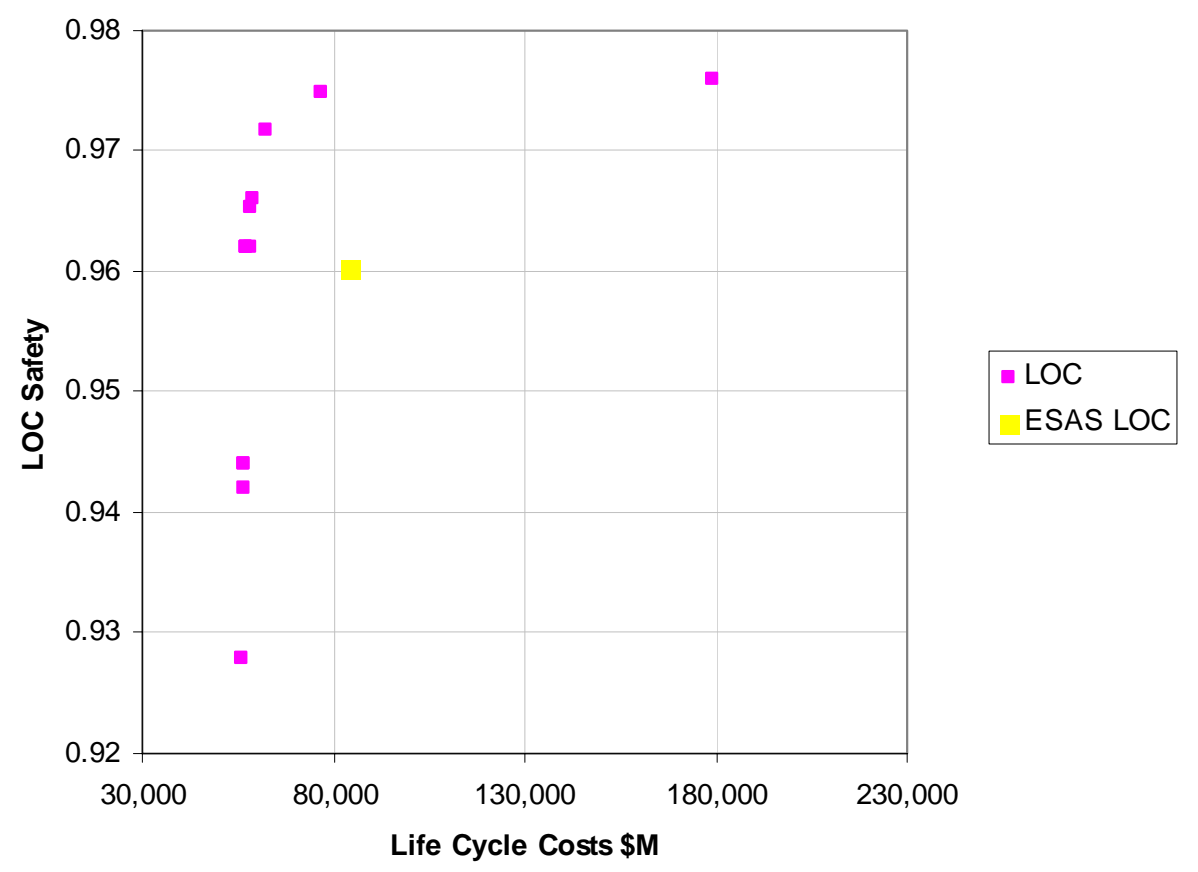

Figure 81: LOC Pareto Frontier for ESAS

As with the Apollo architectures, in this Pareto frontier the LOC safety numbers were calculated for the given LOM Pareto frontier points. The simulation could be reoptimized for LOC, but since the dependencies on LOM and LOC are interrelated and the Pareto frontier is of similar shape, the LOC of the optimized LOM points was used for the Pareto frontier.

An uncertainty analysis can also be performed on the optimal Pareto frontiers for both LOM and LOC. To accomplish this uncertainty analysis, a Monte Carlo simulation 
was completed. This Monte Carlo simulation was accomplished with triangular distributions on the subsystem reliabilities and catastrophic engine fractions. The resulting 90\% confidence (highest cost, lowest reliability) and 10\% confidence (lowest cost, highest reliability) are plotted against the baseline results. A summary of the LOM and LOC results is given as Figure 82 and Figure 83

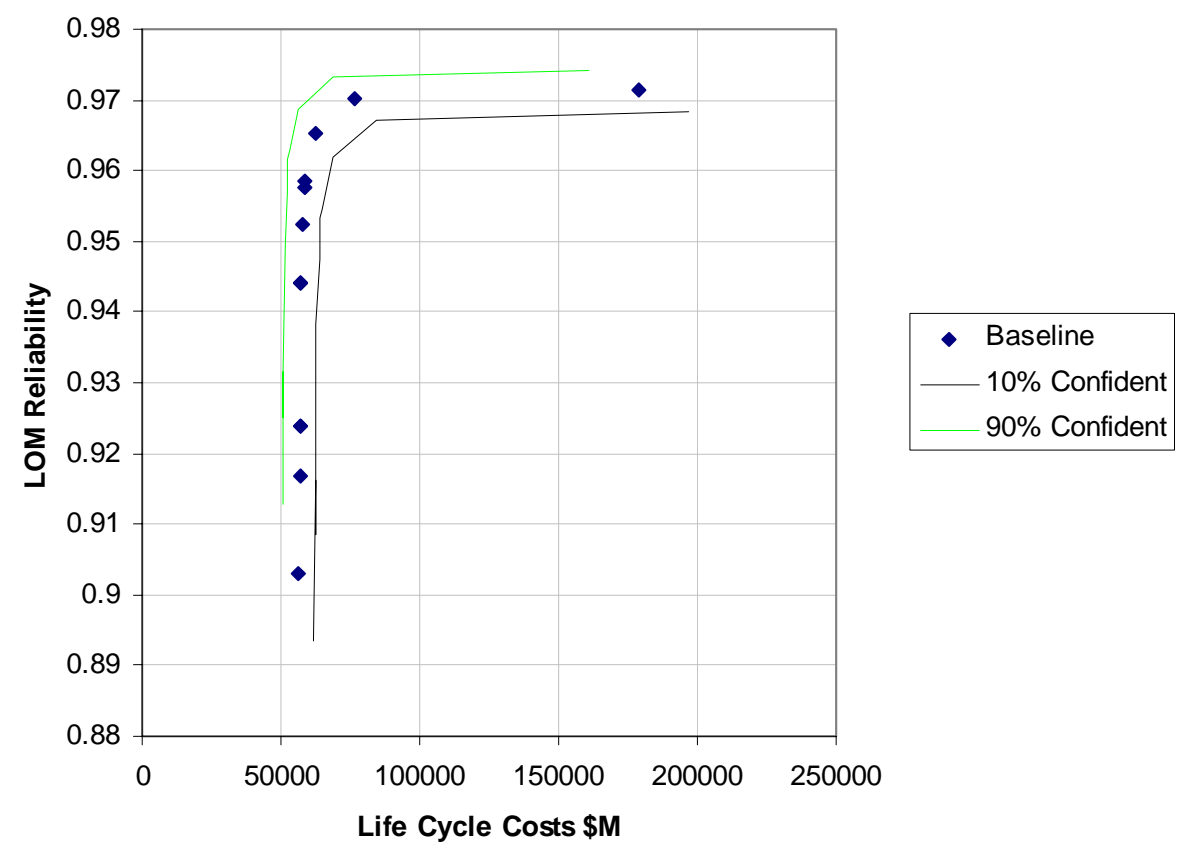

Figure 82: ESAS LOM Pareto Frontier Uncertainty 


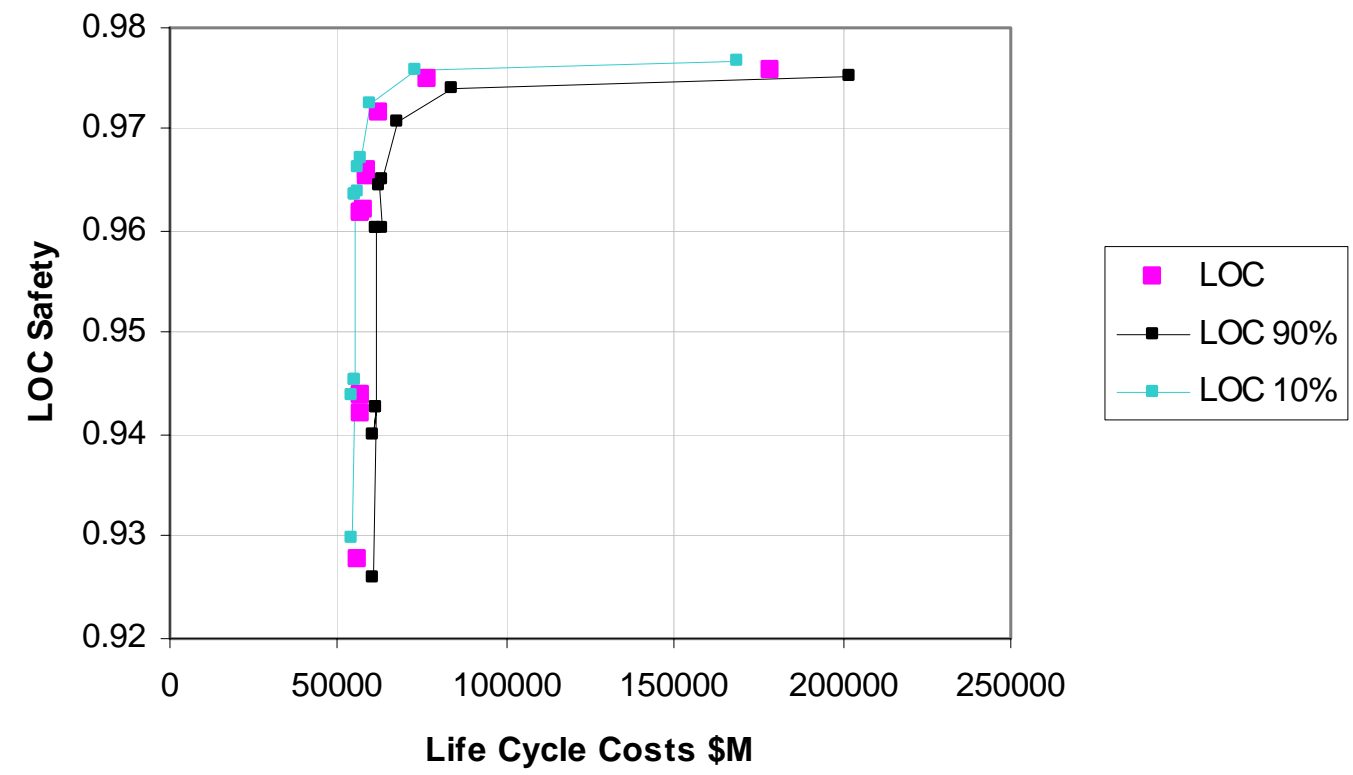

Figure 83: ESAS LOC Pareto Frontier Uncertainty

The resulting Pareto frontiers and their uncertainties can now be presented to the decision maker to demonstrate how increasing or decreasing the cost of the system affects the reliability and the safety of the system. As shown with the Apollo baseline, when the decision maker is not bogged down with the details of the performance, large savings in cost and reliability can result.

\subsection{SUMmary OF ESAS LUNAR ARCHITECTURE SELECTION}

In section 5.5, the Apollo architecture modes were found to be suboptimal. This sub-optimality was attributed to the design methodology and the schedule constraint placed upon the design team. The ESAS architecture study was also found to be suboptimal. The ESAS architecture achieved a suboptimal solution in much the same way as the Apollo architecture. The same trade study methodology was used to evaluate 
the politically driven baseline case, and the trade studies off of that baseline did not investigate the whole design space. The method proposed in this research identifies the ideal set of solutions outside the political biases of the design team. The resulting ESAS architecture is $\$ 27$ Billion less expensive over the entire life of the program. A summary of the ESAS savings is given as Table 60.

Table 60: Pareto Frontier and ESAS Baselines Comparison

\begin{tabular}{lccccc}
\hline$\$ M$ & $\begin{array}{c}\text { ESAS } \\
\text { Final }\end{array}$ & $\begin{array}{c}\text { ESAS } \\
\text { (Cost) }\end{array}$ & $\%$ Diff & $\begin{array}{c}\text { ESAS } \\
(\text { Rel) }\end{array}$ & $\%$ Diff \\
DDTE & $\$ 31,941$ & $\$ 31,538$ & $1 \%$ & $\$ 20,075$ & $37 \%$ \\
TFU & $\$ 2,399$ & 2,064 & $14 \%$ & $\$ 1,550$ & $35 \%$ \\
LCC & $\$ 84,514$ & $\$ 76,760$ & $9 \%$ & $\$ 57,104$ & $32 \%$ \\
LOM & 0.9427 & 0.9701 & $3 \%$ & 0.9440 & $0 \%$ \\
LOC & 0.9602 & 0.9749 & $2 \%$ & 0.9619 & $0 \%$ \\
\hline
\end{tabular}

Table 61: Pareto Frontier and ESAS Baselines Failure Rate Comparisons

\begin{tabular}{lccc}
\hline$\$ M$ & ESAS & ESAS & $\%$ Diff \\
& Final & (Cost) & \\
LOM & 0.0573 & 0.0299 & $48 \%$ \\
LOC & 0.0398 & 0.0251 & $37 \%$ \\
\hline
\end{tabular}

In this table, the ESAS (Cost) column denotes the optimal ESAS architecture that has approximately the same cost as the ESAS baseline. The ESAS (Rel) column denotes the ESAS architecture that has approximately the same reliability as the ESAS architecture. Table 61 shows the percentage decrease in the failure rate as opposed to the traditional 
reliability percent difference. This is a true measure in the reduction of failures due to the increased reliability of the system.

As these results show, the Pareto Frontier method eliminates the biases of the designers and the decision maker and presents only the important design criteria to the decision maker (cost, reliability, and safety). The results are up to $40 \%$ reduction of cost while maintaining the equivalent reliability and 50\% reduction in failure rate keeping the same costs.

A second advantage is that the family of solutions is presented to the decision maker. Because this Pareto frontier plots the entire range of solutions as the proposed budget changes, the solution for the optimal reliability is already solved. This allows the decision maker to make choices based not only on his point solution budget, but also based on his projected project budget. 


\title{
CHAPTER 6
}

\section{LUNAR ARCHITECTURE MODE SELECTION AND}

\begin{abstract}
ANALYSIS
Chapter 5 described the usefulness of the methodology presented in this thesis to solve the ideal architecture elements for a given lunar architecture mode. Once the optimal architectures are selected through the Pareto frontier, it naturally follows that the different lunar modes can be compared to find the ideal lunar architecture mode, as well as the ideal elements in the mode to produce the ideal lunar campaign. This campaign selection method is a useful extension of the architecture analysis presented in the previous chapter. Chapter 6 describes the method to compare the different lunar modes and portrays the results of this analysis for the Apollo lunar mode selection.
\end{abstract}

\subsection{Comparison of Apollo Lunar ARCHITECTURE SELECTION}

The three Pareto frontiers calculated for the Apollo lunar architecture modes can be presented to the decision maker on the same axis. The combination of these modes will show the decision maker the difference between the optimal architecture elements of the different lunar architecture mission modes. This analysis will then be compared with the Apollo mission mode analysis of the early 1960s.

As noted early in this thesis, the Apollo LOR mission mode was selected by NASA as the preferred way to travel to the moon. This decision was made based upon the LOR mode having the smallest vehicles and therefore the highest performance 
margins. LOR also offered similar costs and reliabilities to the other lunar modes considered. Chapter 5 described how the more optimal solutions could be obtained for all three mission modes. These ideal Pareto frontiers can then be compared to see if the Apollo decision changes as a result of the comparison of the ideal solutions. This combination of Pareto frontiers is shown as Figure 84.

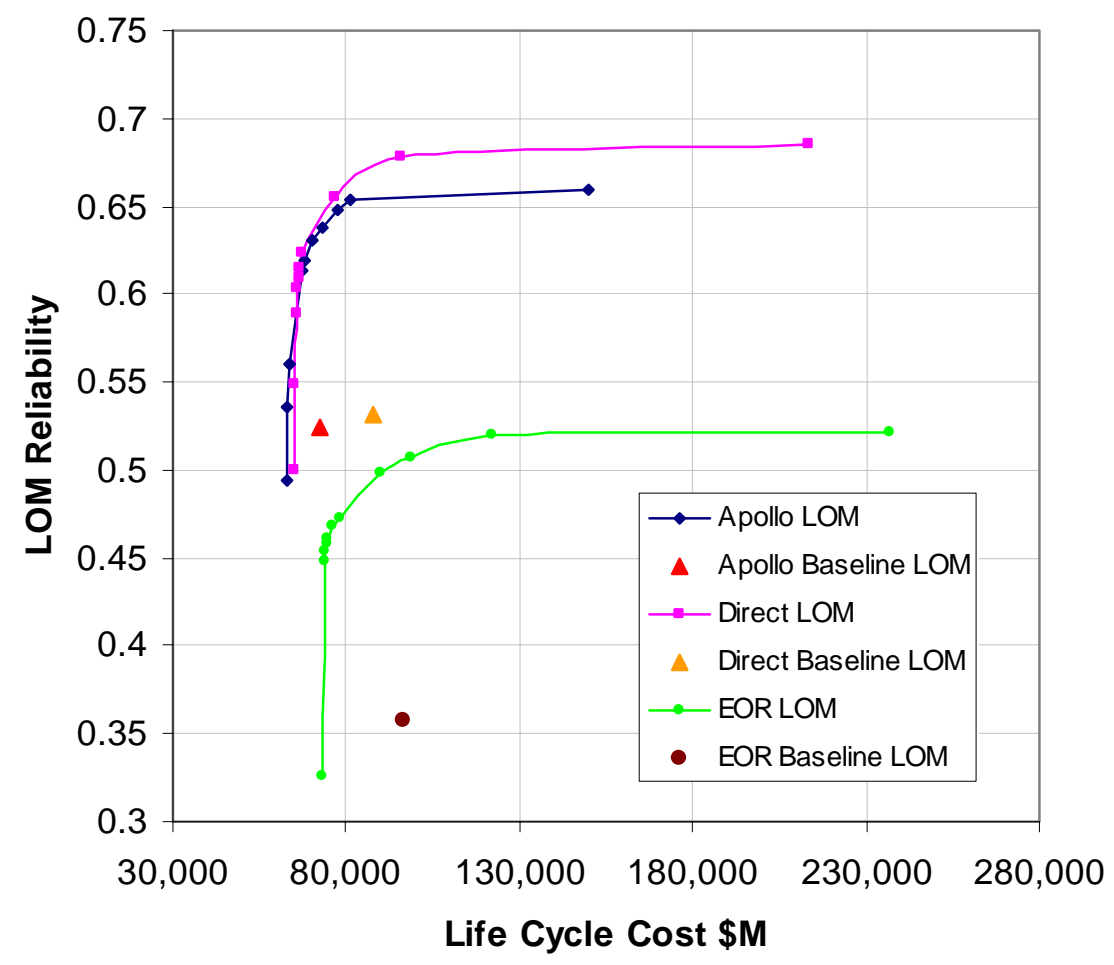

Figure 84: Mission Mode Selection for Apollo Lunar Architectures LOM

As this figure indicates, the Apollo LOR and Apollo Direct mission modes both have similar LOM reliabilities for the low cost optimal solutions. As the LCC increases and the reliability increases, the Direct mission mode results in a higher reliability than the LOR mission mode. The EOR mission mode has the same spacecraft mission as the 
Direct but breaks the launch into two separate launch vehicles. This results in significantly lower mission reliability than both the Direct and EOR mission modes.

As this mission mode selection shows, the solutions for the Pareto frontiers have the same general trend as the original Apollo baselines in the Apollo mission mode selection of the 1960s. The LOR and Direct have similar LOM reliabilities yet the Direct has a slightly higher cost. The EOR mission mode, treated as an alternative for the LOR mode, never approaches the reliability of the other two modes and is therefore never considered.

The Apollo Direct Pareto frontier and the Apollo LOR Pareto frontiers are very similar for the low LCC costs. As Figure 85 shows, the LOR mission mode has the least expensive solutions of the 3 mission modes. This low cost is attributed to the low mass the of the LOR architecture when compared with the Direct architecture that must take the entire CM to the surface of the moon. The Direct mission, in turn, has a slightly higher reliability due to the use of a single pressure vessel for the crew and the fact that a lunar orbit rendezvous is not necessary for this mode. As the LCC increases past \$66 B FY2006, the Direct mode becomes the most optimal solution. Unfortunately, due to the nature of the two mission modes, both modes can not be pursued concurrently. Therefore, the decision maker must decide whether the budget is likely to be greater than or less than the $\$ 66 \mathrm{~B}$ crossover. If the budget is likely to be higher the Direct mission should be chosen, but if the budget is likely to be lower, the LOR mission mode should be chosen. 


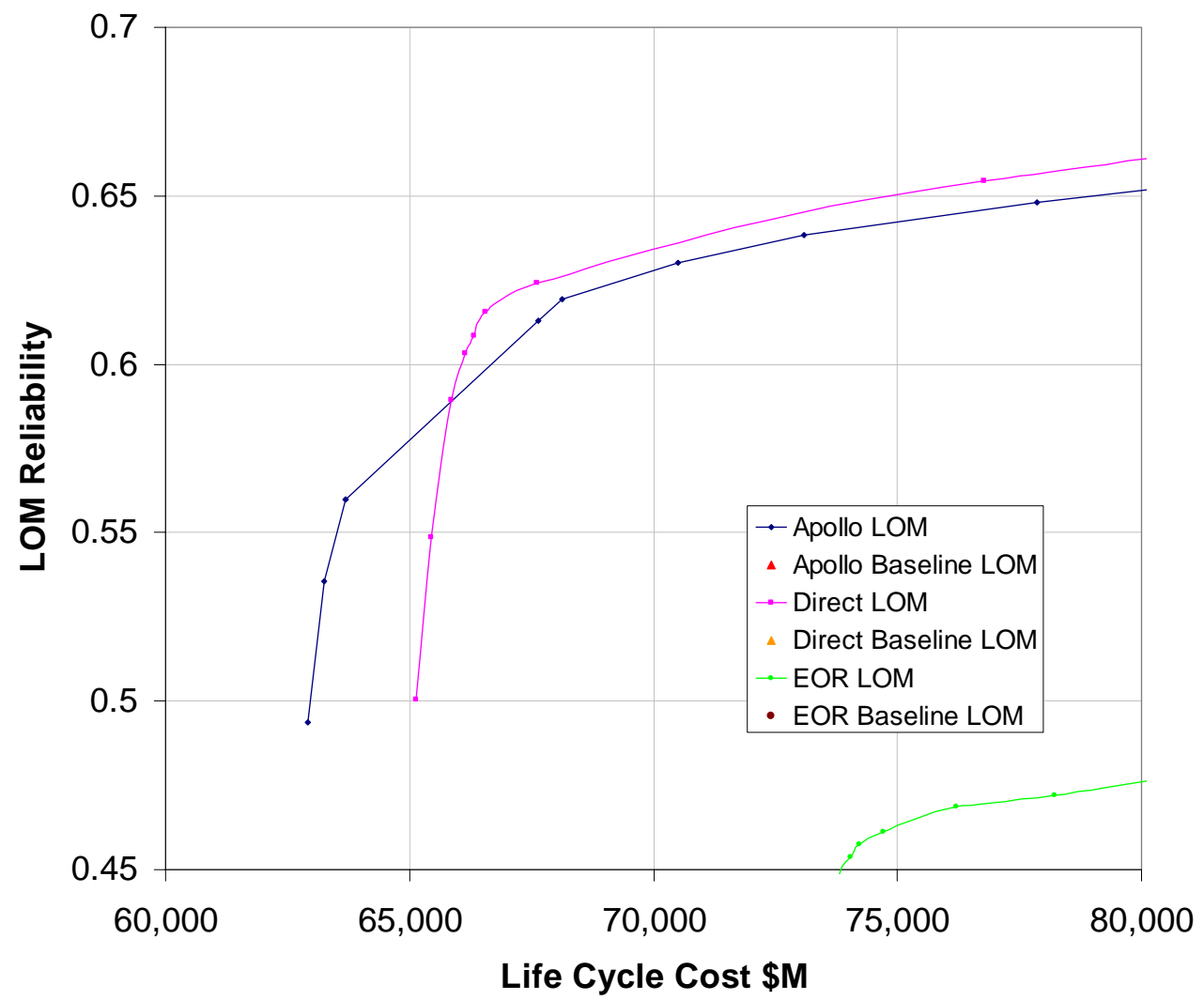

Figure 85: Zoomed in Comparison of Pareto Frontiers for Apollo LOR and Direct

This combination of mission modes shows the decision maker exactly how each mission mode's optimal solution changes with increasing LCC. The mission mode can be chosen based upon the projected budget of the project. This result verifies the Apollo decision maker's solution in the early 1960s. The Apollo LOR mission has the lowest cost for a similar reliability to the direct missions for the low cost solutions.

This combination of Pareto frontiers can also be completed for the LOC safety for the various Apollo lunar architectures. As noted in Chapter 5, these LOC safety numbers are not optimized, but rather based upon the optimal LOM solutions. A summary of the combination of LOC Pareto frontiers is given as Figure 86. 


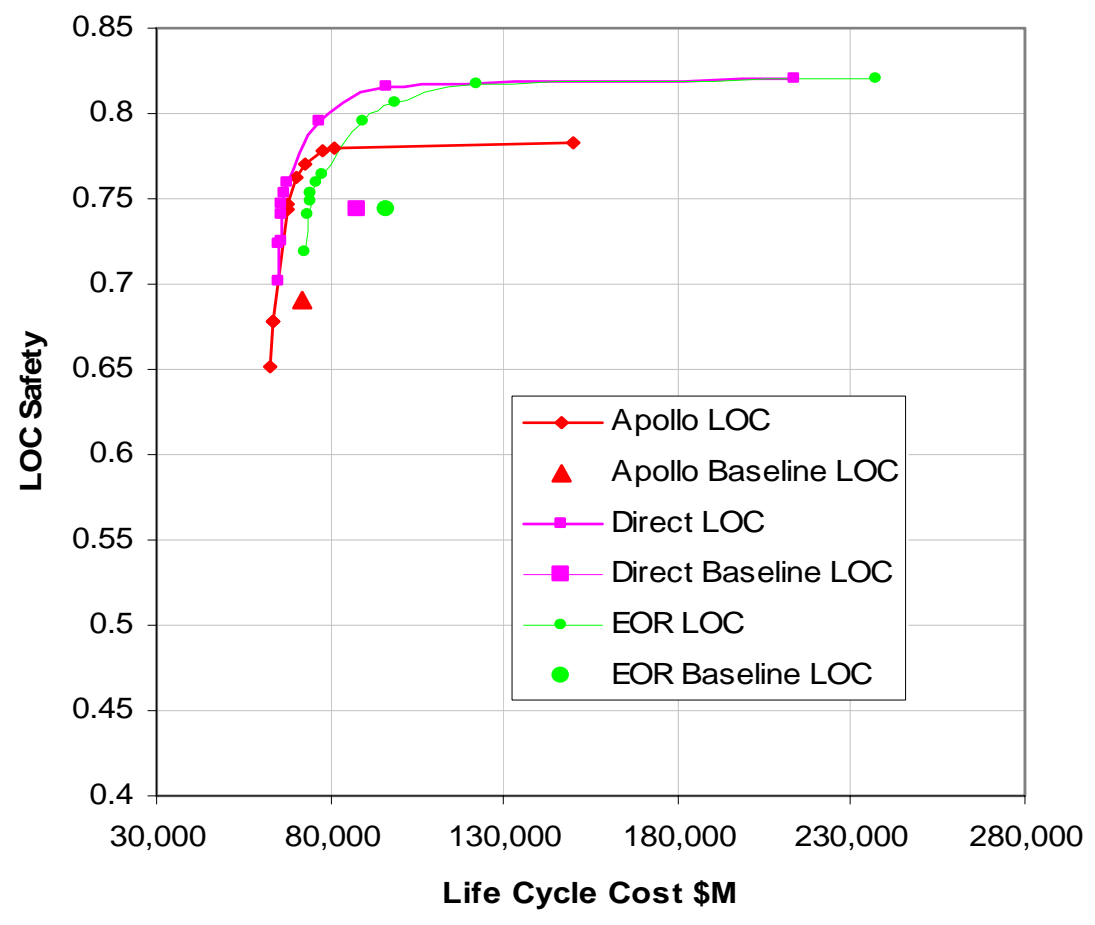

Figure 86: Mission Mode Selection for Apollo Lunar Architectures LOC

This LOC solution is very different than the LOM solution presented as Figure 84. In this model, the difference between the LOC solutions and the LOM solutions is mainly in the EOR architecture. The drastic increase in the LOC over the LOM of the EOR architecture is due to the unmanned launch of the Saturn V-like launch vehicle. The launch vehicle is the lowest reliability system in all of the Apollo lunar architectures. The EOR system requires two of these low reliability launches; and, therefore the reliability of the system is decreased. The LOC safety of each of the missions is not as greatly affected by the launch vehicle due to the crew abort systems, which exist on all crewed launch vehicles considered. Also, the cargo launch of the EOR system has very little impact on the LOC of the system since the cargo launch carries a tanker and 
therefore decreases the safety of the system due to the rendezvous of the tanker and the spacecraft stack in LEO. This affect is somewhat mitigated by the smaller launch vehicle of the EOR architecture when compared with the Direct architecture.

The resulting safety solutions of Figure 86 show that the EOR, although closer than the LOM, still is never the ideal solution for the Apollo system. The LOR is again the solution for the lowest cost system. As the LCC increases, the safety solution quickly changes to the Direct mission. This is because of the low safety associated with two habitats and the lunar orbit rendezvous. It can be argued that the two habitats actually increase the reliability of the system due to the lifeboat nature of the second habitat. The fact that the $\mathrm{CM}$ is a single habitat that is taken to the surface results in a system that is designed to be robust and more survivable than a smaller, lower margin habitat on the LM.

\subsection{UnCERTAINTy ANALySIS APPLIED To ARCHITECTURE SELECTION}

The lunar mode selection problem is similar to the lunar architecture selection problem in that uncertainty can be applied to the systems to determine the ideal solution with uncertainty bands on the mission modes. As with the individual Pareto frontiers, the $90 \%$ and $10 \%$ confidence bands will be applied to the lunar architecture modes to determine the best and the worst case scenarios. As with the original Pareto frontiers, triangular distributions were placed on the subsystem reliabilities and catastrophic engine failure rates. A Monte Carlo simulation was then completed on the models by running 10,000 points for each element in the optimized architecture. The $10 \%$ confidence solution is defined as the design point where $10 \%$ of the costs are below and $10 \%$ of the reliabilities are higher. This is considered the best case solution. The $90 \%$ confidence 
solution is defined as the design point where $90 \%$ of the costs are below and $90 \%$ of the reliabilities are above the baseline. This is considered the worst case solution. These uncertainties can be plotted with the baseline solutions to determine the most robust lunar mode solution with respect to uncertainty. The best case scenarios for LOM are presented as Figure 87.

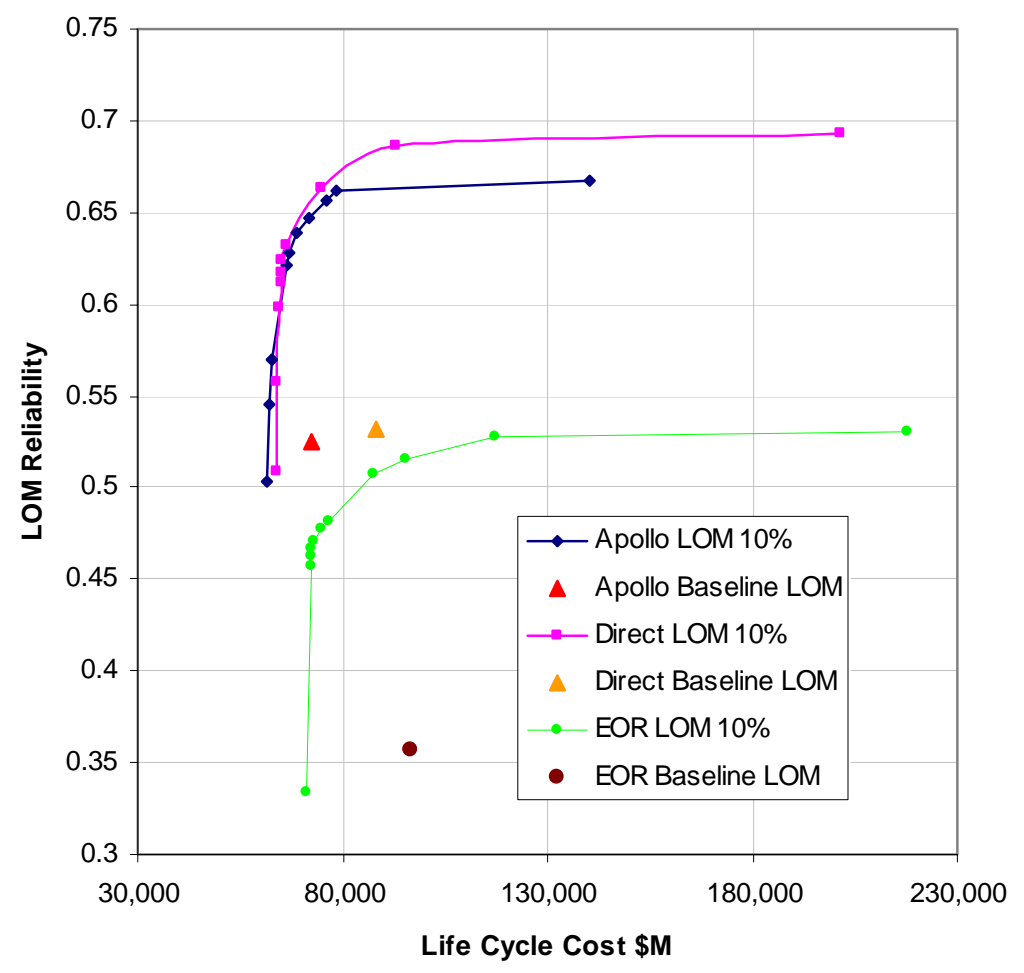

Figure 87: Best Case LOM Scenario for Apollo Lunar Modes

As this figure shows, the best case scenario solution is very similar to the baseline solution. The costs are significantly lower and the reliabilities are higher but the relative answers between the different mission modes are the same. The lowest cost solution is still the LOR and the Direct solution quickly overtakes the LOR as the LCC increases 
past $\$ 64$ Billion. This is slightly lower than the $\$ 67$ Billion of the baseline mission. The worst case scenario is also similar in trends to the baseline solution. The worst case LOM scenarios are presented as Figure 88.

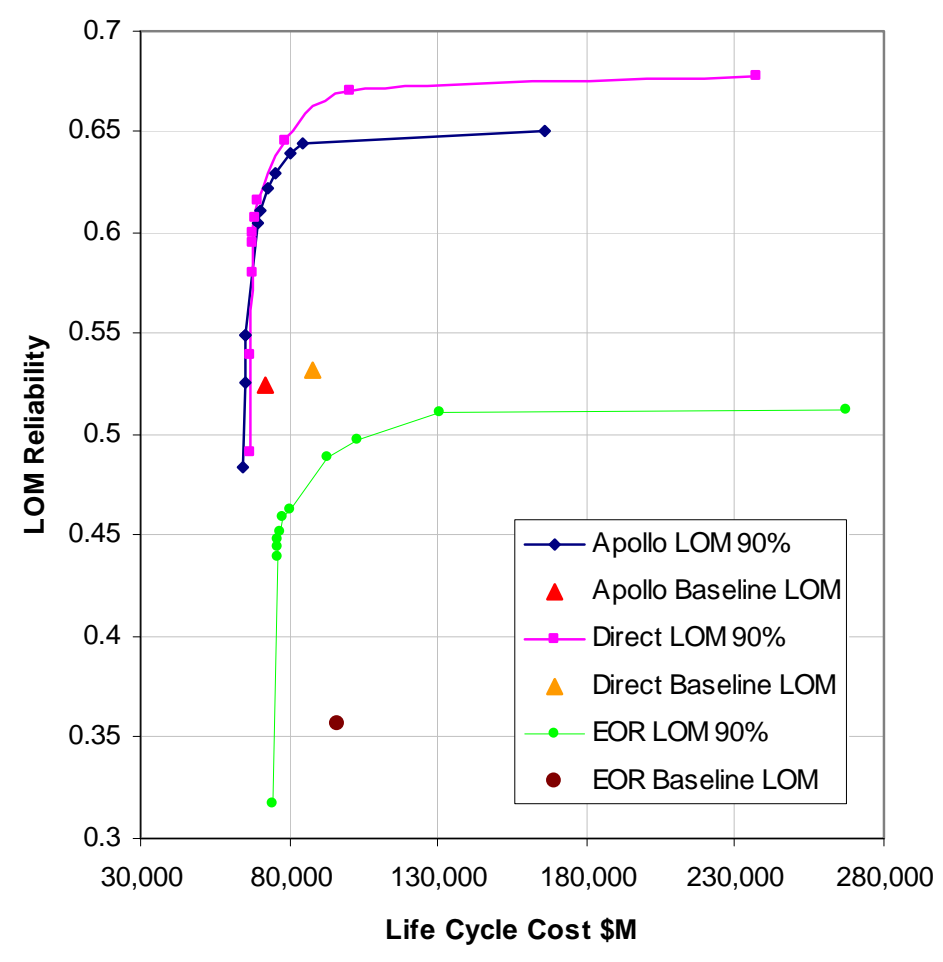

Figure 88: Worst Case LOM Scenarios for Apollo Mission Modes

As noted for the best case scenario, the worst case scenarios have the same trends as the baseline mission mode selections. The crossover point for the Direct and LOR missions is now at a higher $\$ 67.4$ Billion. The comparison of the crossover points is given as Table 62. 
Table 62: Comparison of Crossover Points for Apollo LOR and Direct Mission Modes

\begin{tabular}{lcc}
\hline & LCC & Reliability \\
Best Case & $\$ 64,628$ & 0.5985 \\
Worst Case & $\$ 67,445$ & 0.5807 \\
Baseline & $\$ 65,684$ & 0.5896 \\
\hline
\end{tabular}

As this table shows, the crossover point changes slightly due the Monte Carlo uncertainty calculations.

Due to the small differences between the LOR and Direct mission modes, the combination of the best and worst case scenarios can be a useful selection tool for the selection of the mission modes. If the uncertainty bands overlap a definitive selection cannot be made between the modes. A summary of these mission mode comparisons based upon uncertainty is given as Figure 89. As this figure shows, the uncertainty bands for LOR and Direct overlap for the low LCC solutions. This shows that both LOR and Direct are equally good solutions for the low cost mission. As the LCC increases, the Direct mission becomes significantly higher than the LOR mission. Therefore as the LCC increases past \$ 90 B the Direct worst case scenario has a higher solution than even the best case LOR solution. 


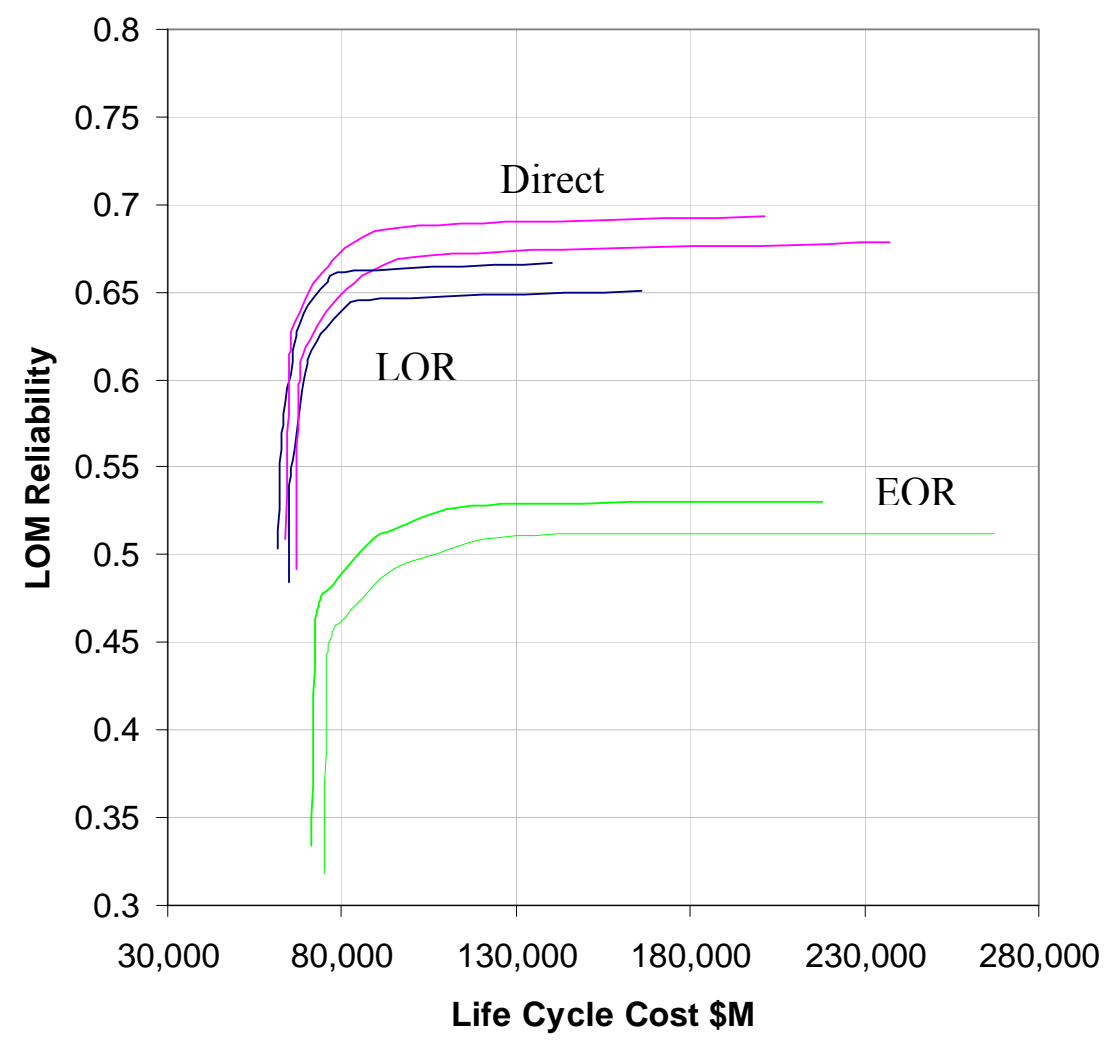

Figure 89: Uncertainty Bands for Apollo Mission Mode Selections

The same analysis can be completed for the best and worst case scenarios for the LOC for the Apollo mission modes. This analysis is completed based upon a Monte Carlo simulation of 10,000 runs of each element in the optimized Pareto frontier solutions. This simulation is actually the same as the LOM simulation since the LOC numbers are based upon the reliabilities of the subsystems and the different configurations in the ROSETTA models. A summary of the best and worst case scenarios for the LOC of the Apollo mission modes is given as Figure 90. 


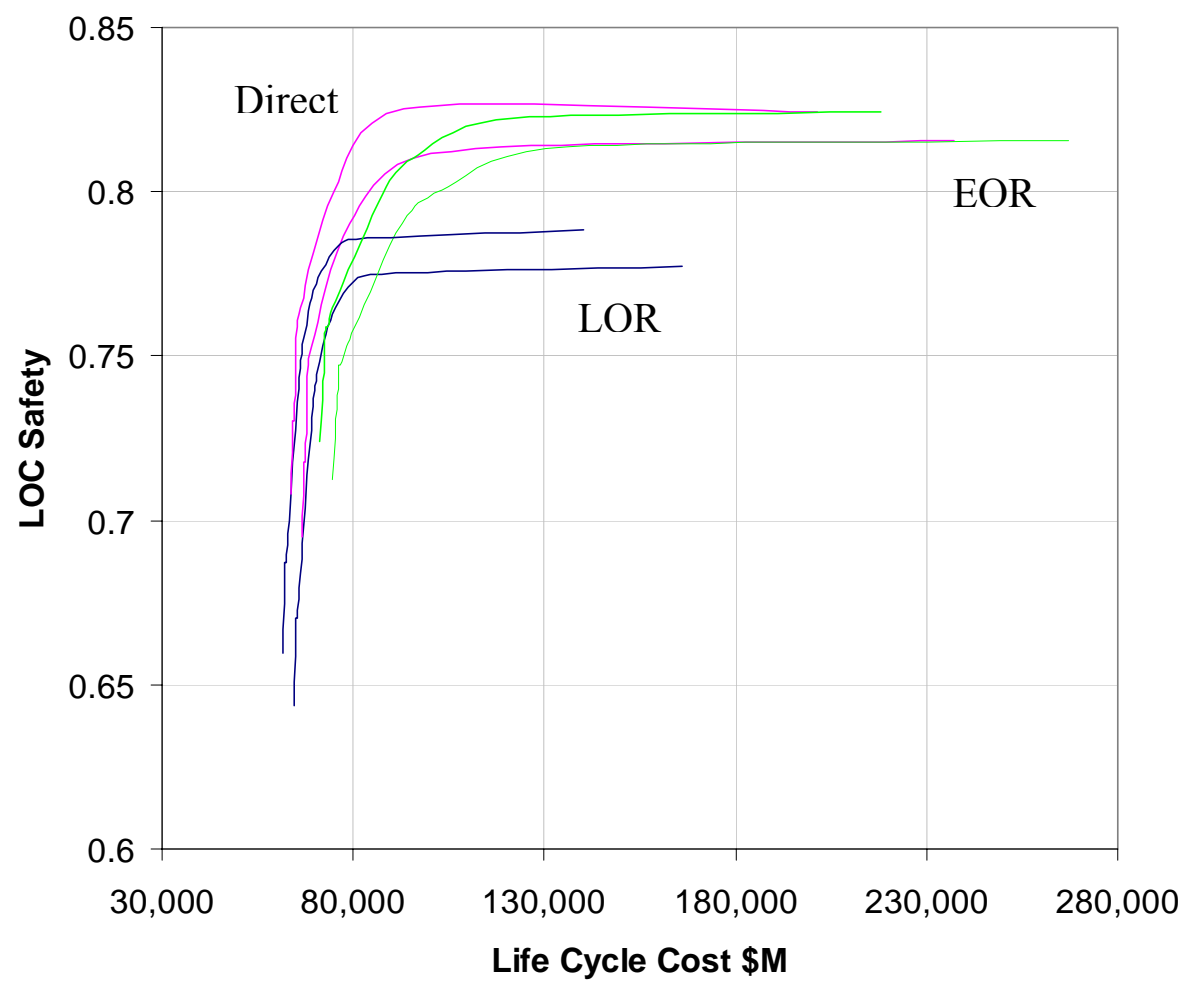

Figure 90: Comparison of Uncertainty Bands for LOC of Apollo Mission Modes

As this figure shows, the LOR mission safety is still the only low cost solution for optimal points. As the LCC increases, the Direct mission mode and the LOR overlap and therefore either mission mode can be selected. As the LCC increases, the Direct becomes the clear LOC solution from $\$ 75 \mathrm{~B}$ to $\$ 100 \mathrm{~B}$ range. As the LCC increase past $\$ 100 \mathrm{~B}$, both the EOR and Direct mission modes uncertainties make either mode a viable solution for the optimal Pareto frontiers. The Direct mode is very flat from the \$100 B LCC range and up. The configuration elements change but the optimal LOC solution is then driven to the $\$ 100$ B solution. 


\subsection{Lunar Mode Selection Via Pareto Frontiers}

As described in the previous sections, different mission modes can be evaluated via the combination of the Pareto frontiers. These Pareto frontiers can then be combined to find the optimal mission mode for a given LCC range. The mission mode solution obtained with these Pareto frontiers is very similar to the solution obtained in the 1960s Apollo mission mode evaluation. The LOR architecture provides the highest LOM for the low cost LCC solutions. As the LCC is increased, the mission mode solution became the Direct mission. Unfortunately, the Direct mission was considered unfeasible due to large launch vehicle needed and the schedule constrictions of the Apollo era [7]. This constraint forced the EOR architecture to be considered. This architecture had a significantly higher cost and lower reliability than the LOR architecture. The infeasibility of the Direct mission with respect to schedule, and the low reliability and high cost of the EOR mission led to the choice of the LOR architecture for the Apollo mission mode. 


\section{CHAPTER 7}

\section{CONCLUSIONS}

The goal of this research was to develop a lunar architecture selection tool that would revolutionize the design process by presenting the decision maker with the ideal cost and reliability solutions. This goal was accomplished through the adaptation of the current design process to include fast acting parametric ROSETTA models with dynamically changing cost and reliability analyses. These new parametric tools were then combined in an integrated framework to evaluate the optimal Pareto frontiers for a given lunar mode. The developed Pareto frontiers present the optimal cost and reliability solutions to the decision maker without bogging down the decision maker with the performance details of the architecture elements.

\subsection{CONCLUSIONS}

The objectives of this research were specified in three different goals set out in section 1.3. The first goal is restated below:

\section{- Goal 1: Create a methodology to bring design discriminators, such as cost and reliability to the forefront of the design process.}

This goal was accomplished by completing a survey of existing design techniques and adapting these techniques to create an optimal Pareto frontier of the costs and reliabilities of a given architecture. These Pareto frontiers present the decision maker with the top 
level design discriminators of cost and reliability without cluttering the decision with specific performance design decisions. These design decisions are automatically chosen by the optimizer to find the ideal cost and reliability solutions for a given weighting on the overall evaluation criteria.

- Goal 2: Create and validate a tool that can quickly and efficiently evaluate lunar architectures to provide accurate reliability and cost information to the decision maker.

This goal was achieved through the combination of ROSETTA models and the creation of a dynamically changing reliability and cost analysis. The approximation of the performance of each of the elements of the architectures was obtained using a response surface methodology of the existing legacy conceptual design tools. The dynamically changing fault trees were created to accurately model the reliability and the safety of each of the vehicle elements as the configurations changed. Each of these vehicles ROSETTA models was then combined in an integrated framework to quickly pass the performance, cost, and reliability variables between the different architecture elements. A life cycle costing tool was also included to calculate the costs of each architecture configuration for a generic lunar campaign. Once the architecture was closed for a given set of design variables, a genetic algorithm is used to find the optimal set of design variables for a given weighting on the overall evaluation criteria. The weighting on the OEC were then changed to create the Pareto frontier for the given architecture mode. A summary of this methodology is given as Figure 91. 


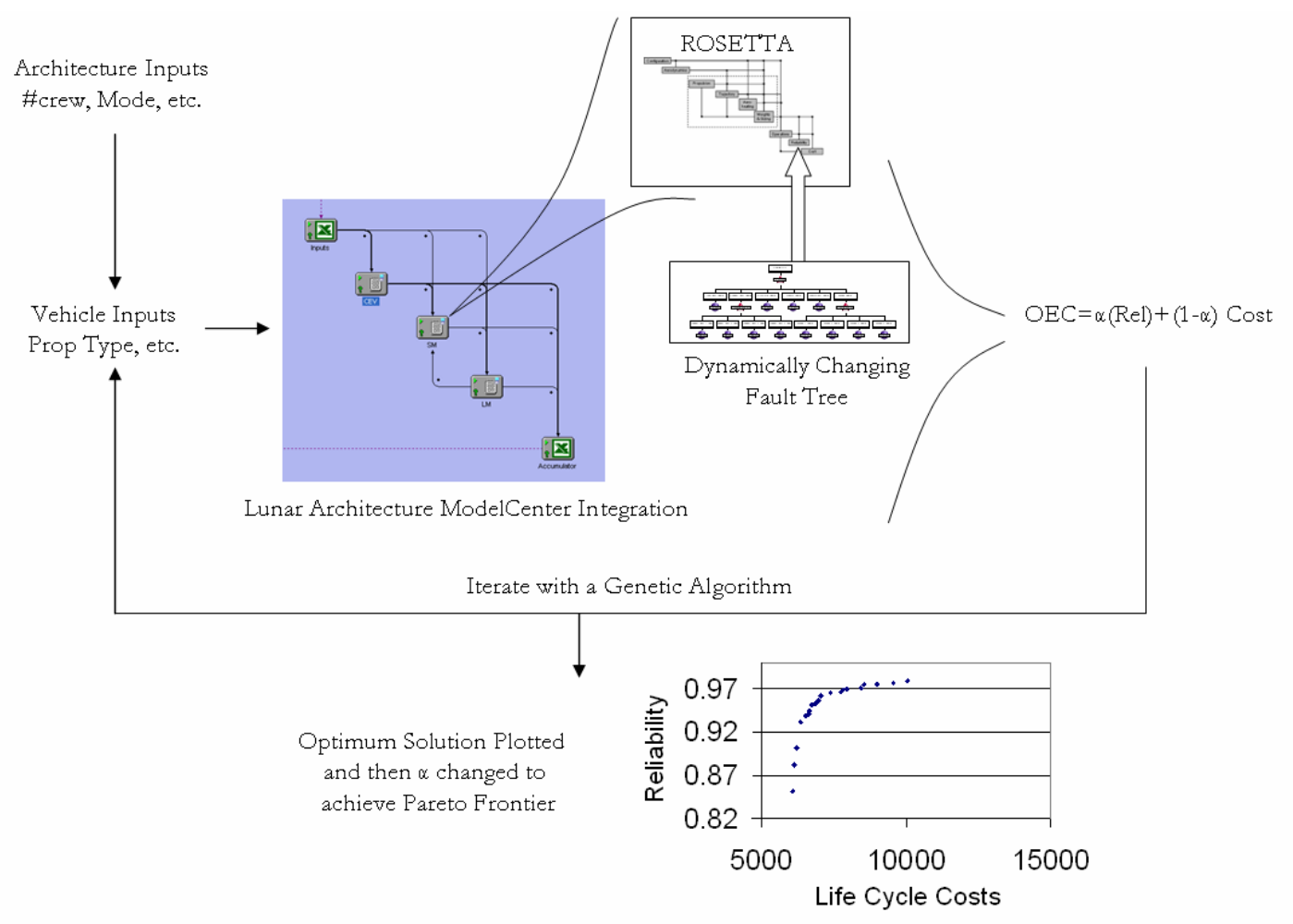

Figure 91: Integrated Lunar Architecture Selection Tool (Revisited)

This method is validated against historical lunar architectures (Apollo) as well as current design studies (ESAS). The results of the tool are within $2 \%$ for the mass, reliability and costs of the lunar architectures.

- Goal 3: Apply the tool to existing lunar architecture modes so that the optimal reliability lunar mode can be evaluated for changing life cycle costs

This third goal was evaluated for the three Apollo architectures considered in the Apollo mode selections as well as the ESAS final report architecture. A series of optimal Pareto solutions were found for each of the lunar architecture modes. In each mode, a 
Pareto frontier was found that exceeded the reliability estimates and cost goals. A summary of the cost savings or reliability increases that can be obtained using this methodology is given as Table 63 .

Table 63: Differences Pareto Frontiers and Baselines

\begin{tabular}{lcccccccc}
\hline$\$ M$ & LOR & $\%$ Diff & EOR & $\%$ Diff & Direct & $\%$ Diff & ESAS & Diff \\
LCC & $\$ 8,958$ & $12 \%$ & $\$ 23,582$ & $25 \%$ & $\$ 22,536$ & $26 \%$ & $\$ 27,410$ & $32 \%$ \\
LOM & 0.11 & $21 \%$ & 0.14 & $39 \%$ & 0.15 & $28 \%$ & 0.0274 & $3 \%$ \\
LOC & 0.08 & $12 \%$ & 0.05 & $7 \%$ & 0.07 & $9 \%$ & 0.0147 & $2 \%$ \\
\hline
\end{tabular}

As this tables shows, the cost savings to achieve the same reliability as the baseline is at least $\$ 8.9$ B FY2004. The reliability gains can be on the order of $20-40 \%$ for reliability and $7-10 \%$ for safety on the Apollo modes. The increase in reliability and safety is smaller for the ESAS architecture because the ESAS system has included reliability analysis in the design methodology and the baseline already has a high reliability. Cost savings of up to $32 \%$ over the baselines can be achieved with the application of this methodology.

These point solutions do not fully convey the power of this methodology. The Pareto frontier created can present the decision maker with all the possible optimal cost, reliability, and safety solutions. Therefore, the decision maker is not constrained to a single cost solution. All of the cost solutions for a mission mode are presented; and, therefore the architecture can be chosen based upon the projected budget as well as the current budget solution. 
Optimizing a single lunar mode can be achieved, but this method can be implemented for multiple mission modes to find the ideal architecture and mission mode for a lunar campaign. This method of decision making is new because it can provide the decision maker with a comparison of the different ideal architectures for the mission modes. The ideal mission mode can then be determined from this analysis. This was verified with the Apollo lunar mission mode selection results. It was found that even with the new optimized solutions, the Apollo LOR has the best low LCC solutions. The Apollo Direct mission becomes the optimal solution as the LCC increases. The EOR solution never enters as the optimal solution.

This mode analysis changed slightly as LOC was calculated. For these Pareto frontiers, the EOR and Direct analyses had almost identical LOC numbers due to the commonality of the vehicles used in the architectures. The LOR solution was only optimal for the minimal LCC, while the Direct mission was optimal for all LCC greater than $\$ 65 \mathrm{~B}$.

Uncertainty analysis is added to the Pareto frontiers to find the most robust, optimal solution. This uncertainty analysis proved that at the lowest cost solutions either LOR or Direct could be chosen. As the LCC increases, the Direct mission mode and the LOR overlap and therefore either mission mode can be selected. As the LCC increases, the Direct becomes the clear LOC solution from $\$ 75 \mathrm{~B}$ to $\$ 100 \mathrm{~B}$ range. As the LCC exceeds $\$ 100 \mathrm{~B}$, both the EOR and Direct mission modes uncertainties make either mode a viable solution for the optimal Pareto frontiers. The Direct mode is very flat from the $\$ 100$ B LCC range and up. The configuration elements change, but the optimal LOC solution is then driven to the $\$ 100 \mathrm{~B}$ solution. 
In conclusion, this method can accurately and quickly evaluate lunar architectures. The dynamic calculations of performance, cost, and reliability allow the decision maker to be presented with information about the cost and reliability of multiple optimal solutions. These optimal solutions, presented as a Pareto frontier, can be used to select the best lunar architecture and mission mode for any design budget. The resulting solutions can save between $\$ 9$ and $\$ 27$ B for the same reliability when compared over a 32 flight lunar campaign. For the same cost, this method can increase the reliability from $3 \%$ to $28 \%$. These reliability numbers are somewhat misleading due to the high reliability of the ESAS architecture. The 3\% reliability increase for the ESAS LOM translates into a $48 \%$ decrease in the failure rate of the ESAS architecture.

\subsection{RECOMMENDATIONS FOR FUTURE WORK}

This methodology produced a series of ideal solutions for different lunar architectures. These solutions, presented as a Pareto Frontier, allow the decision maker to view the ideal reliability as a function of the life cycle cost of the system. This methodology was verified and validated against the Apollo mission modes and the ESAS final mission mode. Although successful, there are certain areas of this process that can be improved. These improvements are enumerated below:

\section{- $\quad$ Evaluate other potential lunar campaigns.}

Although the ESAS and each of the Apollo mission modes were considered in this research, there are plenty of other potential candidate campaigns to consider for future lunar initiatives. This includes changing the number of crew and lunar stay 
durations to evaluate which lunar campaign could provide the lowest cost and highest reliability for other lunar campaigns.

\section{- Include safety in the overall evaluation criteria and optimize.}

This improvement would add a third axis to the lunar architecture selection tool. For this research, LOC was treated as a byproduct of the reliability of the system. This method worked well for the architectures considered, but as the lunar campaigns and lunar architectures become more exotic, it may become necessary to optimize with LOC included in the OEC. This addition would add a third axis to the Pareto frontiers and would somewhat complicate the presentation to the decision maker. Because of this complication, the inclusion of safety in the optimization should only be considered if it adds to the information the decision maker needs to choose an ideal architecture.

\section{- $\quad$ Evaluate the scientific benefit of a lunar architecture.}

This improvement would add a second layer to the lunar architecture selection tool. The scientific benefit of a mission can be treated as a benefit of the lunar campaign which would allow the architecture level variables to be traded based upon increasing the scientific benefit with cost, safety, and reliability as constraints. This would allow the scientific benefit to be optimized, while the Pareto frontiers created in this analysis can be treated as constraints. 


\section{- $\quad$ Add the time value of money to the life cycle cost calculator}

This simple addition to the method would allow the different funding profiles to be compared. The timeframe of the investments would affect the selection of the ideal lunar architecture. Although this did not affect the lunar architectures evaluated in this research, other architectures could have competing DDT\&E and TFU costs. This improvement would account for the difference between high DDT\&E and high TFU architectures.

\section{- $\quad$ Add a operations cost model to the ROSETTA models}

This improvement to the cost model would complete the life cycle costing model. This improvement will allow the total costs of the lunar architecture to be calculated. This change will allow the cost model to include facility changes and workforce changes necessary to implement the lunar architecture. 
APPENDIX A

\section{Lunar Architecture Tool Simulation Results}

\begin{tabular}{|c|c|c|c|c|c|c|c|c|c|c|c|c|c|}
\hline & & Rel & $90 \%$ Rel & $80 \%$ Rel & $70 \%$ Rel & $60 \%$ Rel & $50 \%$ Rel & $40 \%$ Rel & $30 \%$ Rel & $20 \%$ Rel & $10 \%$ Rel & Cost & Apollo \\
\hline \multirow{6}{*}{ 兊 } & Structure & $\pi$ & Al & Al & Al & Al & Al & Al & Al & Al & Al & Al & Al \\
\hline & RCS & Hypergolic & Hypergolic & Hypergolic & Hypergolic & Hypergolic & Hypergolic & Hypergolic & Hypergolic & Hypergolic & Hypergolic & Hypergolic & Hypergolic \\
\hline & Avionics Red & 1 & 1 & 1 & 1 & 1 & 1 & 1 & 0 & 0 & 0 & 0 & 0 \\
\hline & ECLSS Red & 1 & 1 & 1 & 1 & 1 & 0 & 0 & 0 & 0 & 0 & 0 & 0 \\
\hline & Power Red & 1 & 1 & 1 & 1 & 0 & 0 & 0 & 0 & 0 & 0 & 0 & 0 \\
\hline & Parachute Red & 1 & 0 & 0 & 0 & 0 & 0 & 0 & 0 & 0 & 0 & 0 & 0 \\
\hline & Propellant Type & LOX/CH4 & LOXICH4 & Hypergolic & LOX/CH4 & LOXICH4 & $\mathrm{LOX} / \mathrm{CH} 4$ & LOX/LH2 & LOX/LH2 & LOX/LH2 & LOX/LH2 & LOX/LH2 & Hypergolic \\
\hline & Engine Type & Expander & Expander & Pressure & Expander & Expander & Expander & Expander & Expander & Expander & Expander & Expander & Pressure \\
\hline & Structure Type & $\mathrm{Ti}$ & Al & Al & Al & Al & Al & Al & Al & $\mathrm{Al}$ & Al & Al & Al \\
\hline क & Engine Red & 1 & 1 & 1 & 0 & 0 & 0 & 0 & 0 & 0 & 0 & 0 & 0 \\
\hline & Power Red & 1 & 1 & 1 & 0 & 0 & 0 & 0 & 0 & 0 & 0 & 0 & 0 \\
\hline & Pressurant Mat & $\mathrm{Ti}$ & $\mathrm{Ti}$ & $\mathrm{Ti}$ & $\mathrm{Ti}$ & $\mathrm{Ti}$ & $\mathrm{Ti}$ & $\mathrm{Ti}$ & $\mathrm{Ti}$ & $\mathrm{Ti}$ & $\mathrm{Ti}$ & $\mathrm{Ti}$ & Al \\
\hline & Ascent Fuel Type & Hypergolic & Hypergolic & Hypergolic & Hypergolic & Hypergolic & Hypergolic & Hypergolic & Hypergolic & Hypergolic & Hypergolic & Hypergolic & Hypergolic \\
\hline & Descent Fuel Type & LOX/CH4 & $\mathrm{LOX} / \mathrm{CH} 4$ & LOX/CH4 & $\mathrm{LOX} / \mathrm{CH} 4$ & LOX/CH4 & Hypergolic & LOX/CH4 & Hypergolic & Hypergolic & Hypergolic & Hypergolic & Hypergolic \\
\hline & TM LS & 2 & 2 & 2 & 2 & 2 & 2 & 2 & 2 & 2 & 2 & 2 & 2 \\
\hline & TM LLO & 2 & 2 & 2 & 2 & 2 & 2 & 2 & 2 & 2 & 2 & 2 & 2 \\
\hline & Engine Red Ascent & 1 & 1 & 1 & 1 & 0 & 0 & 0 & 0 & 0 & 0 & 0 & 0 \\
\hline$\Sigma$ & Engine Red Descent & 1 & 1 & 0 & 0 & 0 & 0 & 0 & 0 & 0 & 0 & 0 & 0 \\
\hline 5 & AR Ascent & 75 & 75 & 75 & 75 & 150 & 150 & 150 & 150 & 150 & 150 & 150 & 75 \\
\hline & AR Descent & 75 & 75 & 150 & 150 & 150 & 139.2857143 & 150 & 128.5714286 & 128.5714286 & 128.5714286 & 139.2857143 & 75 \\
\hline & Ascent Engine Cycle Type & Pressure & Expander & Expander & Expander & Expander & Expander & Expander & Expander & Expander & Expander & Expander & Pressure \\
\hline & Descent Engine Cycle Type & Expander & Expander & Expander & Expander & Expander & Expander & Expander & Expander & Expander & Expander & Expander & Pressure \\
\hline & Pressurant Mat & $\mathrm{Ti}$ & $\mathrm{Ti}$ & $\mathrm{Ti}$ & $\mathrm{Ti}$ & $\mathrm{Ti}$ & $\mathrm{Ti}$ & $\mathrm{Ti}$ & $\mathrm{Ti}$ & $\mathrm{Ti}$ & $\mathrm{Ti}$ & GrEp & $\mathrm{Ti}$ \\
\hline & Structure Type & $\mathrm{Ti}$ & $\mathrm{Al}$ & $\mathrm{Al}$ & Al & $\mathrm{Al}$ & Al & Al & Al & Al & Al & $\mathrm{Al}$ & Al \\
\hline & Stage 1 Engine Type & Gas Gen & Gas Gen & Staged-Comb & Staged-Comb & Staged-Comb & Staged-Comb & $\sqrt{\text { Staged-Comb }}$ & Staged-Comb & Staged-Comb & Staged-Comb & Staged-Comb & Gas Gen \\
\hline & Stage 1 Propellant Type & LOX/RP1 & LOX/RP1 & LOX/RP1 & LOX/RP1 & LOX/RP1 & LOX/RP1 & LOX/RP1 & LOX/RP1 & LOX/RP1 & LOX/RP1 & LOX/RP1 & LOXIRP1 \\
\hline & Stage 1 Structure Type & $\mathrm{Ti}$ & & & Al & $\mathrm{Al}$ & $\mathrm{Al}$ & Al & Al & $\mathrm{Al}$ & Al & Al & Al \\
\hline & Stage 2 Engine Type & Gas Gen & Staged-Comb & Staged-Comb & Gas Gen & Gas Gen & Staged-Comb & Staged-Comb & Staged-Comb & Staged-Comb & Staged-Comb & Staged-Comb & Gas Gen \\
\hline & Stage 2 Propellant Type & LOX/RP1 & LOX/RP1 & LOX/RP1 & LOX/LH2 & LOX/LH2 & LOX/RP1 & LOX/RP1 & LOX/RP1 & LOX/RP1 & LOX/RP1 & LOX/RP1 & LOX/LH2 \\
\hline$\geq$ & Stage 2 Structure Type & $\mathrm{Ti}$ & Al-Li & Al & Al & Al & Al & Al & Al & Al & Al & Al & $\mathrm{Al}$ \\
\hline & Stage 3 Engine Type & Gas Gen & Staged-Comb & Staged-Comb & Gas Gen & Gas Gen & Staged-Comb & Staged-Comb & Staged-Comb & Staged-Comb & Staged-Comb & Staged-Comb & Gas Gen \\
\hline & Stage 3 Propellant Type & LOX/RP1 & LOX/RP1 & LOX/RP1 & LOX/LH2 & LOX/LH2 & LOX/RP1 & LOX/RP1 & LOX/RP1 & LOX/RP1 & LOX/RP1 & LOX/RP1 & LOX/LH2 \\
\hline & & $\mathrm{Ti}$ & $\begin{array}{c}\text { Al-Li } \\
1\end{array}$ & Al-Li & Al & Al & Al & Al & Al & Al & Al & $\mathrm{Al}$ & \\
\hline & $\begin{array}{l}\text { Stage } 1 \text { Engine Out } \\
\text { Stage } 2 \text { Engine Out }\end{array}$ & $\begin{array}{l}1 \\
1\end{array}$ & $\begin{array}{l}1 \\
1\end{array}$ & 1 & 1 & 1 & 1 & 1 & 1 & 1 & 0 & 0 & 0 \\
\hline & $\begin{array}{l}\text { Stage } 2 \text { Engine Out } \\
\text { Stage } 3 \text { Engine Out }\end{array}$ & 1 & $\begin{array}{l}1 \\
1\end{array}$ & $\begin{array}{l}1 \\
1\end{array}$ & $\begin{array}{l}1 \\
1\end{array}$ & $\begin{array}{l}1 \\
1\end{array}$ & $\begin{array}{l}1 \\
1\end{array}$ & $\begin{array}{l}1 \\
0\end{array}$ & $\begin{array}{l}1 \\
0\end{array}$ & $\begin{array}{l}1 \\
0\end{array}$ & $\begin{array}{l}1 \\
0\end{array}$ & $\begin{array}{l}0 \\
0\end{array}$ & $\begin{array}{l}0 \\
0\end{array}$ \\
\hline & Gross Mass & 7,738 & 7,972 & 7,972 & 7,972 & 7,201 & 6,638 & 6,638 & 6,024 & 6,024 & 6,024 & 5,987 & 6024 \\
\hline & Dry Mass & 6,747 & 6,977 & 6,977 & 6,977 & 6,220 & 5,776 & 5,776 & 5,174 & 5,174 & 5,174 & 5,168 & 5174 \\
\hline & DDTE & 9,958 & 9,535 & 9,535 & 9,535 & 9,012 & 8,686 & 8,686 & 6,259 & 6,259 & 6,259 & 6,255 & 6259 \\
\hline & TFU & 231 & 204 & 204 & 204 & 188 & 163 & 163 & 140 & 140 & 140 & 140 & 140 \\
\hline 西 & Total Cost & 10,190 & 9,740 & 9,740 & 9,740 & 9,200 & 8,849 & 8,849 & 6,399 & 6,399 & 6,399 & 6,395 & 6399 \\
\hline $\bar{U}$ & CB LOV & 1 & 1 & 1 & 1 & 1 & 1 & 1 & 1 & 1 & 1 & 1 & 1 \\
\hline & CB LOC & 1 & 1 & 1 & 1 & 1 & 1 & 1 & 1 & 1 & 1 & 1 & 1 \\
\hline & CB DDTE & 9,958 & 9,535 & 9,535 & 9,535 & 9,012 & 8,686 & 8,686 & 6,259 & 6,259 & 6,259 & 6,255 & 6259 \\
\hline & CB TFU & 231 & 204 & 204 & 204 & 188 & 163 & 163 & 140 & 140 & 140 & 140 & 140 \\
\hline & Gross Mass & 26,507 & 27,720 & 31,191 & 20,575 & 19,280 & 18,866 & 14,505 & 14,169 & 14,169 & 14,169 & 14,025 & 24917 \\
\hline & Dry Mass & 7,727 & 8,266 & 7,952 & 4,758 & 4,653 & 4,629 & 4,327 & 4,302 & 4,302 & 4,302 & 4,239 & 5523 \\
\hline & $\begin{array}{l}\text { DDTE } \\
\text { DDTE }\end{array}$ & 6,238 & 5,356 & 5,426 & 4,365 & 4,343 & 4,333 & 4,278 & 4,268 & 4,268 & 4,268 & 4,257 & 4514 \\
\hline & TFU & 260 & 164 & 195 & 123 & 122 & 121 & 121 & 120 & 120 & 120 & 119 & 169 \\
\hline$\sum_{\infty}$ & Total Cost & 6,498 & 5,520 & 5,622 & 4,489 & 4,465 & 4,454 & 4,399 & 4,389 & 4,389 & 4,389 & 4,376 & 4682 \\
\hline & CB LOV & 1 & 1 & 1 & 1 & 1 & 1 & 1 & 1 & 1 & 1 & 1 & 1 \\
\hline & CB LOC & 1 & 1 & 1 & 1 & 1 & 1 & 1 & 1 & 1 & 1 & 1 & 1 \\
\hline & CB DDTE & 6,238 & 5,356 & 5,426 & 4,365 & 4,343 & 4,333 & 4,278 & 4,268 & 4,268 & 4,268 & 4,257 & 4514 \\
\hline & CB TFU & 260 & 164 & 195 & 123 & 122 & 121 & 121 & 120 & 120 & 120 & 119 & 169 \\
\hline & Gross Mass & 14,337 & 14,201 & 12,929 & 12,929 & 11,708 & 12,199 & 11,708 & 12,213 & 12,213 & 12,213 & 12,154 & 16183 \\
\hline & Dry Mass & 4,055 & 4,231 & 3,676 & 3,676 & 3,349 & 3,223 & 3,349 & 3,221 & 3,221 & 3,221 & 3,204 & 4216 \\
\hline & DDTE & 11,036 & 7,015 & 6,567 & 6,567 & 6,249 & 5,655 & 6,249 & 5,653 & 5,653 & 5,653 & 5,645 & 6639 \\
\hline & TFU & 1,278 & 665 & 640 & 640 & 615 & 609 & 615 & 609 & 609 & 609 & 608 & 733 \\
\hline$\sum$ & Total Cost & 12,314 & 7,681 & 7,207 & 7,207 & 6,863 & 6,264 & 6,863 & 6,262 & 6,262 & 6,262 & 6,253 & 7372 \\
\hline & CB LOV & 1 & 1 & 1 & 1 & 1 & 1 & 1 & 1 & 1 & 1 & 1 & 1 \\
\hline & СB LOC & 1 & 1 & 1 & 1 & 1 & 1 & 1 & 1 & 1 & 1 & 1 & 1 \\
\hline & CB DDTE & 11,036 & 7,015 & 6,567 & 6,567 & 6,249 & 5,655 & 6,249 & 5,653 & 5,653 & 5,653 & 5,645 & 6639 \\
\hline & CB TFU & 1,278 & 665 & 640 & 640 & 615 & 609 & 615 & 609 & 609 & 609 & 608 & 733 \\
\hline & Gross Mass & $5,329,494$ & $4,260,028$ & $3,640,152$ & $2,359,303$ & $2,210,576$ & $2,793,673$ & $2,488,652$ & $2,445,466$ & $2,445,466$ & $2,414,226$ & $2,380,747$ & 2936359 \\
\hline & Dry Mass & 286,762 & 248,176 & 235,181 & 175,115 & 166,811 & 187,047 & 170,140 & 167,924 & 167,924 & 157,910 & 155,019 & 181532 \\
\hline & DDTE & 29,125 & 15,039 & 14,201 & 13,555 & 13,241 & 12,870 & 12,552 & 12,401 & 12,401 & 12,385 & 12,347 & 13746 \\
\hline & TFU & 2,507 & 978 & 883 & 815 & 794 & 776 & 738 & 733 & 733 & 713 & 704 & 832 \\
\hline 3 & Total cost & 31,632 & 16,017 & 15,084 & 14,370 & 14,035 & 13,646 & 13,290 & 13,134 & 13,134 & 13,098 & 13,051 & 14578 \\
\hline & CB LOV & 1 & 1 & 1 & 1 & 1 & 1 & 1 & 1 & 1 & 1 & 1 & 1 \\
\hline & CB LOC & 1 & 1 & 1 & 1 & 1 & 1 & 1 & 1 & 1 & 1 & 1 & 1 \\
\hline & CB DDTE & 29,125 & 15,039 & 14,201 & 13,555 & 13,241 & 12,870 & 12,552 & 12,401 & 12,401 & 12,385 & 12,347 & 13746 \\
\hline & CB TFU & 2,507 & 978 & 883 & 815 & 794 & 776 & 738 & 733 & 733 & 713 & 704 & 832 \\
\hline & ASS & 48,582 & 49,893 & 52,093 & 41,476 & $38,18 \mathrm{~s}$ & 37,703 & 32,851 & 32,407 & 32,407 & 32,407 & 32,166 & 47125 \\
\hline & Dry Mass & 18,529 & 19,474 & 18,605 & 15,411 & 14,222 & 13,628 & 13,452 & 12,697 & 12,697 & 12,697 & 12,612 & 14913 \\
\hline & DDTE & 56,358 & 36,946 & 35,729 & 34,023 & 32,844 & 31,543 & 31,764 & 28,581 & 28,581 & 28,565 & 28,504 & 31158 \\
\hline & TFU & 4,276 & 2,011 & 1,923 & 1,783 & 1,719 & 1,670 & 1,637 & 1,603 & 1,603 & 1,583 & 1,571 & 1874 \\
\hline & Total Cost & 60,634 & 38,957 & 37,653 & 35,805 & 34,563 & 33,213 & 33,401 & 30,184 & 30,184 & 30,149 & 30,075 & 33031 \\
\hline$\frac{\bar{v}}{\bar{c}}$ & $\mathrm{CE}$ & 0.6588 & 0.6534 & 0.6479 & 0.6382 & 0.6300 & 0.61 & 0.6130 & 0.55 & 0.5599 & 0.5356 & 0.4939 & 0.5245 \\
\hline$\overline{4}$ & CB LOC & 0.7830 & 0.7803 & 0.7782 & 0.7702 & 0.7619 & 0.7465 & 0.7430 & 0.6786 & 0.6786 & 0.6786 & 0.6515 & 0.6900 \\
\hline & CB DDTE & 56,358 & 36,946 & 35,729 & 34,023 & 32,844 & 31,543 & 31,764 & 28,581 & 28,581 & 28,565 & 28,504 & 31158 \\
\hline & СB TFU & 4,276 & 2,011 & 1,923 & 1,783 & 1,719 & 1,670 & 1,637 & 1,603 & 1,603 & 1,583 & 1,571 & 1874 \\
\hline & Average/Yr & 150,048 & 81,017 & 77,869 & 73,085 & 70,510 & 68,130 & 67,633 & 63,701 & 63,701 & 63,253 & 62,925 & 72210 \\
\hline & OEC & 1.2561 & 1.2103 & 1.1737 & 1.1482 & 1.1304 & 1.1205 & 1.1081 & 1.1138 & 1.1204 & 1.1296 & 1.1476 & 1.0000 \\
\hline
\end{tabular}

Figure 92: Simulation Outputs for Apollo LOR Architecture 


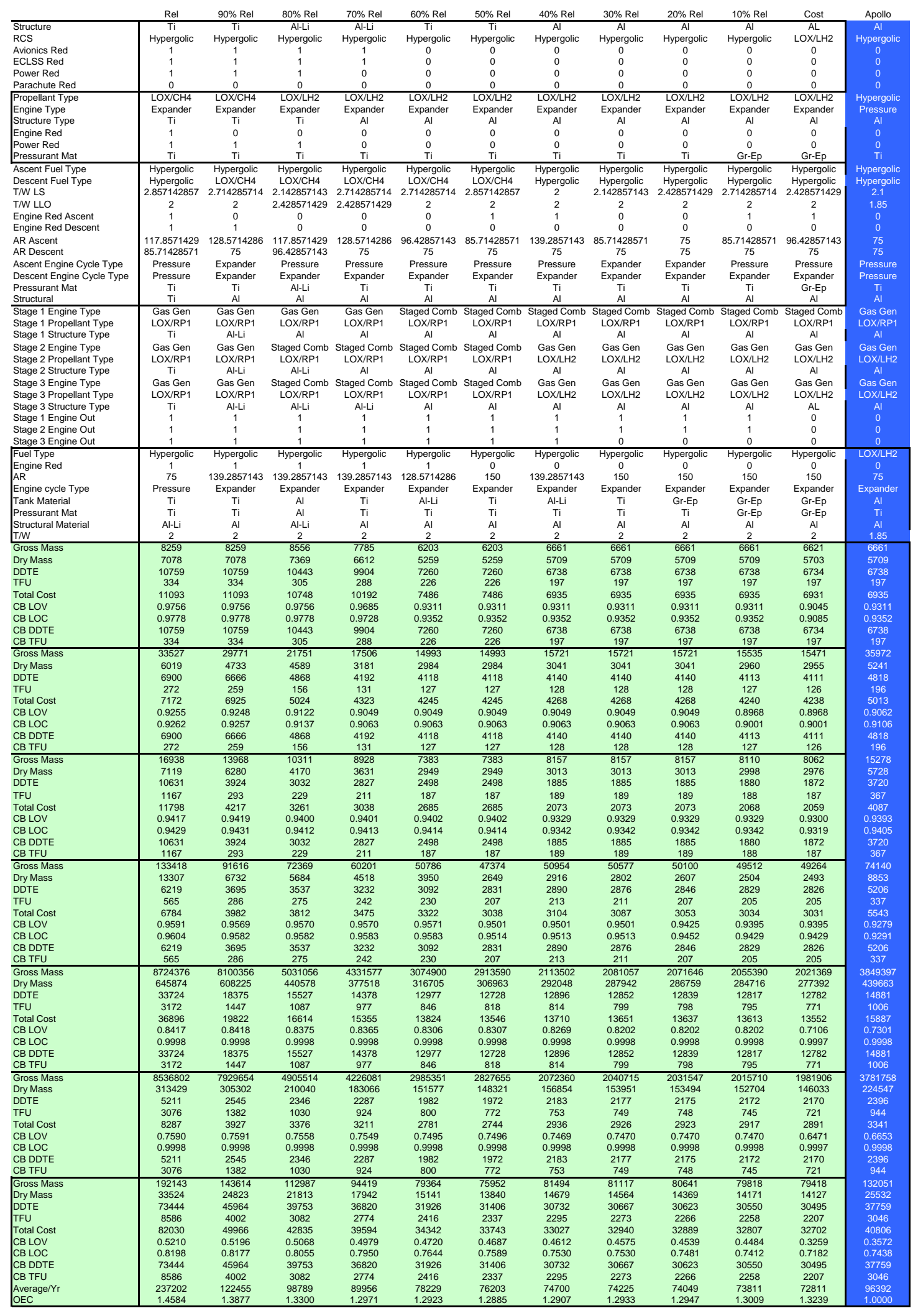

Figure 93: Simulation Outputs for Apollo EOR Architecture 


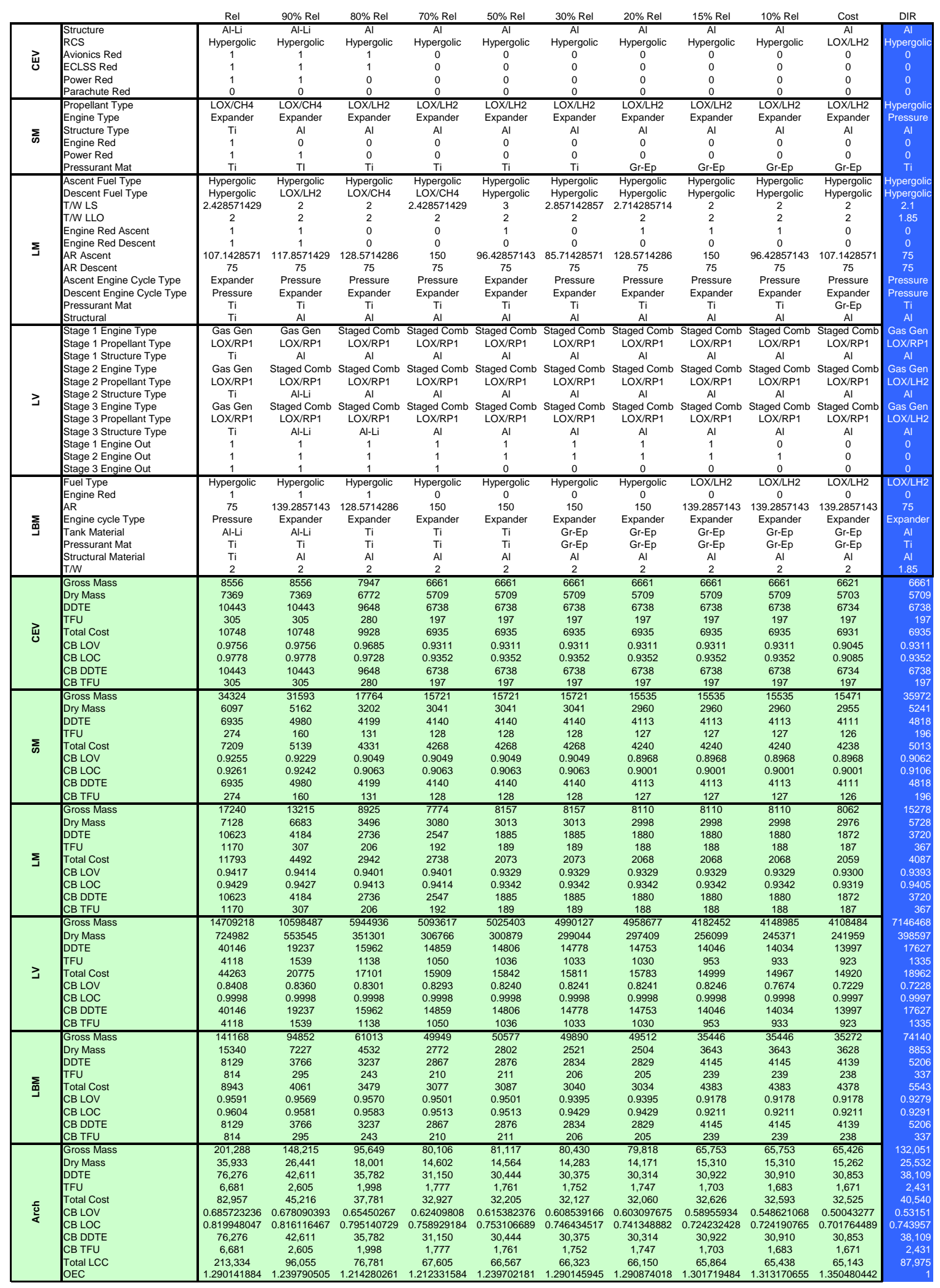

Figure 94: Simulation Outputs for Apollo Direct Architecture 


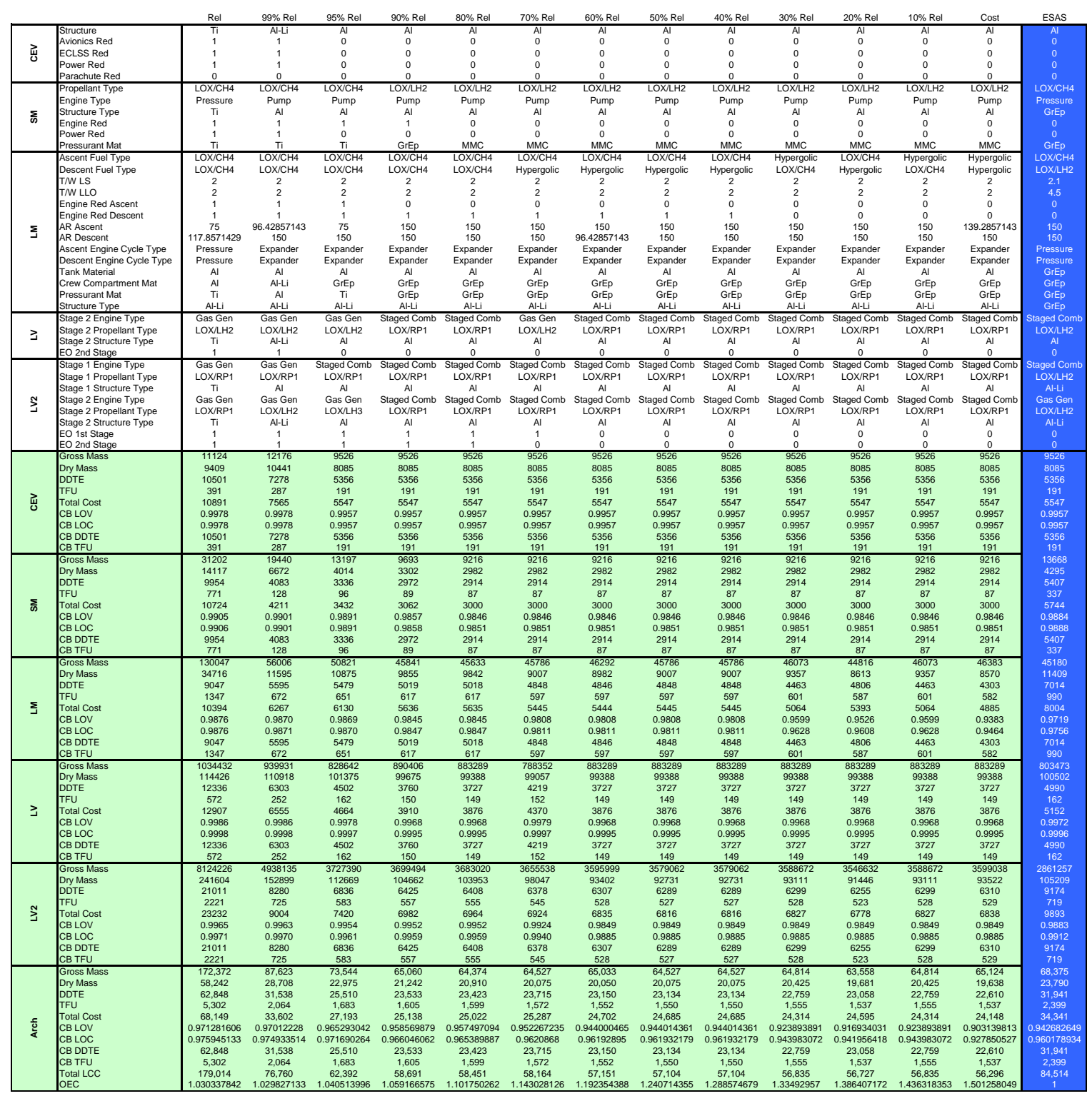

Figure 95: Simulation Outputs for ESAS EOR-LOR Architecture 


\section{APPENDIX B}

\section{Assumed Subsystem Reliability Summary}

Table 64: Engine Subsystem Reliability

\begin{tabular}{lcccc}
\hline Propellant & Cycle & Engine & Reliability & Source \\
LOX/LH2 & Pressure & NA & 0.95 & NA \\
LOX/LH2 & Expander & Rl-10 & 0.996 & 13 \\
LOX/LH2 & Staged Comb & SSME & 0.9985 & 13 \\
LOX/LH2 & Gas Gen & J-2/RS-68 & 0.9987 & 13 \\
LOX/CH4 & Pressure & ESAS & 0.965 & 13 \\
LOX/CH4 & Expander & Rl-10 Deriv & 0.9974 & 13 \\
LOX/RP1 & Staged Comb & RD-180 & 0.998 & NA \\
LOX/RP1 & Gas Gen & F-1 & 0.999 & 69 \\
UDMH/N2O4 & Pressure & Apollo SPS & 0.9969 & 69 \\
UDMH/N2O4 & Expander & NA & 0.99 & NA \\
\hline
\end{tabular}

Table 65: Launch Vehicle Tank Subsystem Reliability

\begin{tabular}{lc}
\hline Tank Type & Structure Rel Baseline \\
Al & 0.99976 \\
Al-Li & 0.99991 \\
Ti & 0.99991 \\
Graphite Epoxy Overwrapped & 0.99975 \\
MMC & 0.999 \\
\hline
\end{tabular}

Table 66: Inspace Tank Subsystem Reliability

\begin{tabular}{lc}
\hline Tank Type & Structure Rel Baseline \\
$\mathrm{Al}$ & 0.9991 \\
$\mathrm{Al}-\mathrm{Li}$ & 0.9991 \\
$\mathrm{Ti}$ & 0.9991 \\
Graphite Epoxy Overwrapped & 0.9975 \\
MMC & 0.99 \\
\hline
\end{tabular}


Table 67: Inspace Structure Subsystem Reliability (SM \& CM)

\begin{tabular}{cc}
\hline Structure Type & Structure Rel Baseline \\
$\mathrm{Al}$ & 0.99999 \\
$\mathrm{Al}-\mathrm{Li}$ & 0.99998 \\
$\mathrm{Ti}$ & 0.99997 \\
Graphite Epoxy Overwrapped & 0.99992 \\
MMC & 0.9999 \\
\hline
\end{tabular}

Table 68: Inspace Structure Subsystem Reliability (LM)

\begin{tabular}{cc}
\hline Structure Type & Structure Rel Baseline \\
$\mathrm{Al}$ & 0.9982 \\
$\mathrm{Al}-\mathrm{Li}$ & 0.9982 \\
$\mathrm{Ti}$ & 0.9982 \\
Graphite Epoxy Overwrapped & 0.995 \\
MMC & 0.99 \\
\hline
\end{tabular}

Table 69: RCS Subsystem Reliability

\begin{tabular}{cc}
\hline Propellant & Pressure Fed \\
LOX/LH2 & 0.995 \\
LOX/Methane & 0.998 \\
UDMH/N2O4 & 0.989 \\
\hline
\end{tabular}

Table 70: Command Module Other Subsystem Reliability Baseline [69].

\begin{tabular}{cc}
\hline Thermal Protection System & 0.99996 \\
Primary Power & 0.99954 \\
Electrical Conversion & 0.99954 \\
Avionics & 0.99954 \\
ECLSS & 0.99825 \\
RCS Propulsion & 0.99985 \\
Landing & 0.99995 \\
\hline
\end{tabular}

Table 71: Service Module Other Subsystem Reliability Baseline [69].

\begin{tabular}{cc}
\hline Avionics & 0.9969 \\
ECLSS & 0.9983 \\
Primary Power Failure & 0.9982 \\
\hline
\end{tabular}


Table 72: Lunar Module Other Subsystem Reliability Baseline [69].

\begin{tabular}{lr}
\hline Landing Leg Failure & 0.99999 \\
Avionics & 0.98756 \\
Electrical Distribution & 0.982 \\
ECLSS & 0.9889 \\
Primary Power Failure & 0.975 \\
Guidance and Navigation & 0.9873 \\
\hline
\end{tabular}

Table 73: Saturn V Other Subsystem Reliability Baseline [69].

\begin{tabular}{lr}
\hline Avionics & 0.968 \\
Electrical Power & 0.9204 \\
Guidance Navigation & 0.9631 \\
\hline
\end{tabular}




\section{REFERENCES}

1 Lewis, R. S., The Voyages of Apollo: The Exploration of the Moon. Quadrangle, New York, NY 1975.

2 Sputnik and The Dawn of the Space Age, Online. Internet 25 June 2006. Available: http://history.nasa.gov/sputnik/

3 John F. Kennedy Presidential Library and Museum Historical Resources, Online. Internet. 20 June 2006. Available:

http://www.jfklibrary.org/Historical+Resources/Archives/Reference+Desk/Speec hes/JFK/Urgent+National+Needs + Page $+4 . h t m$

4 Reeves, D., Scher, M., Wilhite, A., Stanley, D., "The Apollo Lunar Orbit Rendezvous Architecture Decision Revisited," 41st AIAA/ASME/SAE/ASEE Joint Propulsion Conference and Exhibit, Tucson, AZ, July, 2005.

5 Brooks, C., and Ertel, I. The Apollo Spacecraft A Chronology. NASA SP-4009. National Aeronautics and Space Administration. Washington, D.C.

6 "Manned Lunar Landing Operations Analysis and Mode Comparison," NASATM-X-74752, June 1, 1962

7 Logsdon, J. M., "Selecting the Way to the Moon: The Choice of Lunar Orbital Rendezvous Mode," Aerospace Historian, Vol. 18, Summer 1971, pp. 63-71.

8 Apollo 11 Press Kit, Online. Internet 25 June 2006. Available: http://wwwlib.ksc.nasa.gov/lib/presskits.html

9 Project Apollo: A Retrospective Analysis, Online. Internet 25 June 2006. Available: http://history.nasa.gov/Apollomon/Apollo.html

10 Heppenheimer, T.A., The Space Shuttle Decision 1965-1972. Smithsonian Institute Press, Washington, D.C., 2002.

11 David, L., Total Tally of Shuttle Fleet Cost Exceed Initial Estimates, Online. Internet. 25 June 2006. Available:

http://www.space.com/news/shuttle_cost_050211.html

12 Stanley, D., Cook, S., "Exploration System Architecture Study: Overvie of Architecture and mission Operations Approach," Space Ops 2006, Rome Italy June 19-23, 2006.

13 Stanley, D., et. al. Exploration Systems Architecture Study Final Report, Summer 2006. 
14 NASA Report: Columbia Accident Investigation Board Final Report, October 2003.

15 Tam, W., "Improvement Opportunities for Aerospace Design Process," Space 2004 Conference and Exhibit, San Diego, CA, September 28-30 2004.

16 Thompson, R., O’Neil D., "System Architecture Modeling for Technology Portfolio Management using ATLAS," IEEE Aerospace Conference, Big Sky, Montana, March 2006.

17 Smiljanic, R. National Institute of Aerospace Supportability Training, Hampton, VA, May 2006

18 Schrage, D. "Integrated Product and Process Development," Course Notes, 2002.

19 Mavris, D. “Advanced Design Methods,” Course Notes, 2002.

20 Lewis, E. E., Introductions to Reliability Engineering. Wiley and Sons, Inc., New York, NY 1994.

21 Volvoi, V., “Safety by Design” Course Notes, 2006.

22 Kaplan, S. and Garrick, B.J., "On the Quantitative Definition of Risk," Risk Analysis, 1, 11-37, 1981.

23 Department of Defense, NASA, Probabilistic Risk Assessment Guide. 1995.

24 Andrews, J. D., Moss, T. R., Reliability and Risk Assessment. American Society of Mechanical Engineers Press, New York, NY, 2002.

25 Statistical Summary of Commercial Jet Airplane Accidents; Worldwide operations 1959-2004, Airplane Safety Engineering, Boeing Commercial Airplane Ground, May 2005.

http://www.boeing.com/news/techissues/pdf/statsum.pdf

26 Pate-Cornell E., Dillon, R., "Probabilistic Risk Analysis for the NASA Space Shuttle: A Brief History and Current Work" Reliability Engineering and System Safety, 74, 345-352, 2001.

27 NASA Technical Document, "Manned Lunar Landing Mode Comparison," Washington D.C., October 24, 1962.

28 Fragola, J.R., "Space Shuttle Probabilistic Risk Assessment." Proceedings of PSAMIII, Crete, Greece. 1996. 
29 Keisner, A., Olds, J., "Reliability Analysis Technique Comparison, as Applied to the Space Shuttle." AE8900 Special Project, School of Aerospace Engineering, Georgia Institute of Technology, Atlanta, GA, December, 2003.

30 SAIC, Probabilistic Risk Assessment of the Space Shuttle, 1995.

31 Leemis, L. Reliability Probablisitc Models and Statistical Methods. Prentice-Hall, Upper Saddle River, New Jersey, 1995.

32 Young, D., Hutchinson, V., Krevor, Z., Pimentel, J., Reeves, J., Sakai, T., “ Centurion: A Heavy Lift Launch Vehicle Family for Cis-Lunar Exploration.” 40th AIAA/ASME/SAE/ASEE Joint Propulsion Conference and Exhibit, Fort Lauderdale, Fl, July 11-14, 2004.

33 Relex Reliability Suite, Software Package, ver 2.2, Relex Software Corporation, Greensburg, PA, 2004.

34 Liu, T., Chiou, S., “Application of Petri Nets to Failure Analysis," Reliability Engineering and System Safety. Vol 57, 1997. pp. 129-142.

35 Wright, T. P., "Factors Affecting the Cost of Airplanes," Journal of Aeronautical Science. Vol. 3 February, 1936.

36 Department of Defense, Parametric Cost Estimating Handbook, 1995.

37 NASA Johnson Space Center Cost Estimation Guidelines, Online, Internet. June 25, 2006. Available: http://www1.jsc.nasa.gov/bu2/guidelines.html

38 Koelle, Dietrich, "Handbook of Cost Engineering for Space Transportation Systems with TRANSCOST," TRANSCOST Systems, Liebigweg, Germany, 2000.

39 Sova, G., and P. Divan, "Aerodynamic Preliminary Analysis System II, Part II User's Manual,” NASA CR 182077, April 1991.

40 Rowell, L. F., Braun, R. D., Olds, J. R., and Unal, R., "Multidisciplinary Conceptual Design Optimization of Space Transportation Systems," Journal of Aircraft, vol. 35 pp. 218-226, Jan, 1999.

41 Brown, N., Olds, J., "Evaluation of Multidisciplinary Optimization techniques Applied to a Reusable Launch Vehicle," AE 8900 Special Project, School of Aerospace Engineering, Georgia Institute of Technology, Atlanta, GA, May 2004.

42 Braun, R.D., "Collabrative Architecture for Large-Scale Distributed Design," PhD Thesis, Stanford University, Stanford, CA, June, 1996. 
43 Sobieszanski-Sobieski, J., Agte, J., Sandusky, R., Bi-Level Integrated System Synthesis, $7^{\text {th }}$ AIAA/USAF/NASA/ISSMO Symposium on Multidisciplinary Analysis and Optimization, St. Louis, MO, Sept 1998.

44 Simpson, T., "A Concept Exploration Method for Product Family Design." PhD Thesis, School of Aerospace Engineering, Georgia Institute of Technology, Atlanta, GA, September 1998.

45 Yang, K., El-Haik, B., Design for Six Sigma. McGraw-Hill, New York, 2003.

46 Marcus, L., Olds, J. R., et. al., "Technology Assessment for Manned Mars Exploration Using a ROSETTA Model of a Bimodal Nuclear Thermal Rocket (BNTR)," AIAA Space 2001 Conference and Exposition, Albuquerque, NM, August 28-30, 2001.

47 O’Neil, D., Mankins, J., "The Advanced Technology Lifecycle Analysis System (ATLAS)," 2004 International Astronautical Conference, Vancouver, Canada, 2004.

48 Bishop, C.M., and Roach, C. M. "Fast Curve Fitting Using Neural Networks," American Institute of Physics: Review of Science Instruments Vol 63 October 1992

49 Eberhart, R. C. and Dobbins, R. W. Nueral Network PC Tools A Practical Guide. Academic Press, Inc. San Diego, CA 1990.

50 Loch, C. H., M. T. Pich, C. Terwiesch and M. Urbschat: Selecting R\&D Projects at BMW: A Case Study of Adopting Mathematical Programming Models, IEEE Transactions on Engineering Management 48 (1), 2001, 70 - 80.

51 Schmidt, RL, JR Freeland. 1992. Recent progress in modeling R\&D projectselection processes. IEEE Trans. Eng. Management 39(3) 239-246

52 Beaujon, G. J., S. P. Marin., G. C. McDonald. 2001. Balancing and optimizing a portfolio of R\&D projects. Naval Logist. Quart. 48(1) 18-40.

53 Rocket Engine Design Tool for Optimal Performance (REDTOP), Software Package, ver 1.3, Spaceworks Engineering Inc., Atlanta, GA, 2003.

54 Krevor, Z. Space Systems Design Laboratory, Georgia Institute of Technology (2006) Unpublished raw data.

55 Powell, R.W., et. al., "Program to Optimize Simulated Trajectories (POST) Utilization Manual, Volume II, Version 5.2," NASA Langley Research Center, Hampton, VA; Martin Marietta Corporation, Denver, CO., October 1997. 
56 Thompson, R. Space Systems Design Laboratory, Georgia Institute of Technology (2006) Unpublished raw data.

57 Rohrschneider, R., "Development of a Mass Estimating Relationship Database for Launch Vehicle Conceptual Design," AE8900 Special Project, School of Aerospace Engineering, Georgia Institute of Technology, April 26, 2002.

58 Willoughby, L. "A Semi-empirical Method for Propellant Tank Weight Estimation," $27^{\text {th }}$ Annual Conference of the Society of Aeronautical Weight Engineers, Inc. New Orleans, LA 1968.

59 Humble, R., Henry, G., and Larson, W. Space Propulsion Analysis and Design. McGraw-Hill Companies New York, NY. 1995.

60 Mosleh, A. et al. "Procedure for Treating Common-Cause Failures in Safety and Reliability Studies," U.S. Nuclear Regulatory Commission, NUREG/CR-4780, Vol. I and II, Washington, DC. 1988.

61 Spaceflight now. 2006. STS-114 Shuttle Report. 28 Feb 2007. $<$ http://spaceflightnow.com/shuttle/sts114/index2.html $>$

62 Fleming, K. N., Molesh, A. "Common-Cause Data Analysis and Implications in System Modeling," Procedures Int'l Topical Meeting on Probabilistic Safety Methods and Applications, Feb 1985. Vol 1.

63 Tang, Zhihua, Dugan, Joanne. "An Integrated Method for Incorporating Common Cause Failures in System Analysis," Annual Reliability and Maintainability Symposium 2004 Proceedings, LA, January 2004.

64 Modarres, M. What Every Engineer Should Know About Reliability and Rist Analysis. Marcel Dekker, Inc. New York, NY 1993.

65 Bradford, J., "Rocket Engine Design Tool for Optimal Performance (REDTOP-2) Software Manual" ver 1.82006.

66 Huang, Z. Fint, J., Kuck, F. "Key Reliability Drivers of Liquid Propulsion Engines and A Reliability Model for Sensitivity Analysis." $41^{\text {st }}$ AIAA/ASME/SAE/ASEE Joint Propulsion Conference. Tucson, AZ. July 2005.

67 Hark, F., Maggiom G., Sen, D. "A Methodology to Rapidly and Effectively Assess the Reliability of Conceptual Advanced Rocket Engines." 40th AIAA/ASME/SAE/ASEE Joint Propulsion Conference. Fr. Lauderdale, FL. July 2004.

68 Metal Matrix Composites 3M US. 2007. March 3, 2007 < http://solutions.3m.com/wps/portal/3M/en_US/EnergyAdvanced/Materials/Industry_Solutions/MMC/> 
69 NASA Technical Document, "Apollo Reliability and Quality Assurance Program Quarterly Status Report,” Washington D.C., October 8, 1965.

70 NASA Air Force Cost Model, Software Package, ver. 2004, SAIC, Huntsville, AL, 2004.

71 SpaceshipOne, the Ansari X Prize and the Material of the Civilian Space Race. 3 May 2005<http://www.tms.org/pubs/journals/JOM/0411/Byko-0411.html>

72 Mettas, Adamantios. "Reliability Allocation and Optimization for Complex Systems." IEEE Annual Reliability and Maintainability Symposium. Los Angeles, CA 2000.

73 ModelCenter, Software Package, ver 6.1.1, Phoenix Integration Inc., Philadelphia, PA, 2007.

74 NASA Technical Document, "Mass Estimating and Forecasting for Aerospace Vehicles Based on Historical Data JSC-26098," Houston, TX. November, 1994.

75 NASA Technical Document, "Manned Lunar Landing Program Mode Comparison TMX 74929,” Washington, DC. July, 1962. 


\section{VITA}

David Anthony Young was born in Rome, NY on September $6^{\text {th }}, 1980$. He attended elementary school at Gansevoort Elementary in the Rome City School District. He later attended Strough Junior High school, also in Rome. After graduating in the top ten of his class at Rome Free Academy in June, 1998, David attended Clarkson University in Potsdam, NY. In May of 2002, he graduated the top of his class with a Bachelor of Science in Aeronautical Engineering and a Bachelor of Science in Physics. The following fall, Dave enrolled at The Georgia Institute of Technology to pursue graduate studies in Aerospace Engineering. Funded by a National Science Foundation fellowship, Dave received his Master's Degree in Aerospace Engineering from Georgia Tech in May, 2004 and continued toward his Doctoral degree in the same field.

During his Master's studies at Georgia Tech, Dave worked with Dr. John Olds in the Space System Design Laboratory (SSDL). In the SSDL, Dave specialized in conceptual launch vehicle design and trajectory simulation. Following his Masters Dave began his research for his dissertation under the direction of Dr. Alan Wilhite. Under the mentorship of Dr. Olds and Dr. Wilhite, Dave led several design studies ranging from lunar landers to hypersonic reusable launch vehicles. To supplement Dave's research, he worked several summers in both government and industry jobs. Dave has worked at Air Force Research Laboratory in Rome, NY; Lockheed Martin in Denver, CO; NASA Headquarters in Washington, DC; and the Tauri Research Group in Alexandria, VA. He also spent time working closely with NASA Marshall Space Flight Center in Huntsville, $\mathrm{AL}$ as the Georgia Tech team leader for the Advanced Technology and Lifecycle Analysis System (ATLAS) project. Dave is a member of the American Institute of Aeronautics and Astronautic and the Tau Beta Pi National Engineering Honor Society. 